\title{
Plate tectonics in computational simulations of terrestrial mantle convection with grain-size-dependent rheology.
}

\author{
Dissertation \\ zur Erlangung des Doktorgrades \\ der Mathematisch-Naturwissenschaftlichen Fakultäten \\ der Georg-August-Universität zu Göttingen
}

vorgelegt von

Christian Auth

aus Essen

Göttingen 2001 
D7

Referent: Prof. Dr. U. Christensen

Korreferent: Prof. Dr. A. Tilgner

Tag der mündlichen Prüfung: 


\section{Contents}

1 Abstract and Outline of the thesis $\quad 1$

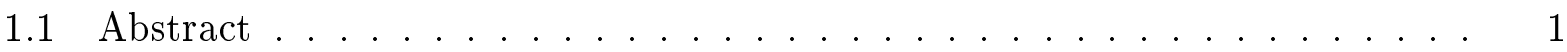

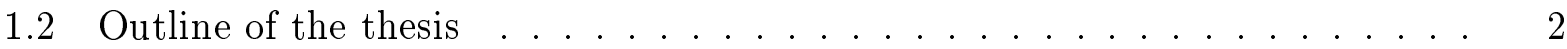

2 Introduction $\quad \mathbf{5}$

2.1 Plate tectonics on Earth . . . . . . . . . . . . . . . 6

2.2 Numerical Models . . . . . . . . . . . . . . . . . 7

2.2.1 Criteria for the evaluation of plate-like behavior in numerical simulations 7

2.2.2 Review of plate-like behavior in previous numerical models . . . . . . 8

2.3 Aim of these thesis . . . . . . . . . . . . . . . . 13

3 The Model $\quad 15$

3.1 Hydrodynamic Equations . . . . . . . . . . . . . . . . . . . 16

3.2 Rheology Equations . . . . . . . . . . . . . . . . 17

3.3 Discussion of the Rheology Equations . . . . . . . . . . . . . . 18

3.3.1 Self-lubrication neglecting the advection of damage . . . . . . . . 18

3.3.2 Influence of the advection of damage . . . . . . . . . . . . . 20

3.3.3 Influence of the damage on the velocity: A simple example . . . . . . 21

4 The numerical treatment of the model equations 25

4.1 Numerical methods . . . . . . . . . . . . . . . . . . . . . 26

4.1 Discretization . . . . . . . . . . . . . . . . . . . . . . . . . . .

4.1.2 Solution of the discretized equations . . . . . . . . . . . . 27

4.2 Test of the Algorithm . . . . . . . . . . . . . . . . . . 28

4.3 Numerical limitations . . . . . . . . . . . . . . . . . . . . 28

4.4 Resolution of the Low Viscosity Zones . . . . . . . . . . . . . . . . 30

4.4.1 Grid-points per LVZ . . . . . . . . . . . . . 30

4.4 .2 Under-resolved LVZs . . . . . . . . . . . . . . . . . 31

4.5 Diagnostic Parameters . . . . . . . . . . . . . . . . 34

5 Bottom heated models $\quad 37$

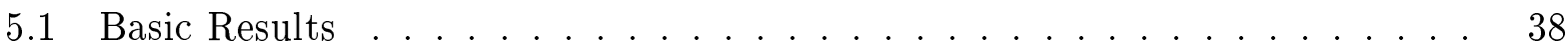


5.1 Introduction . . . . . . . . . . . . . . . 38

5.1 .2 Variation of $a$, series A0 . . . . . . . . . . . 39

5.1.3 Simultaneous variation of $a$ and $b$, series AB . . . . . . . . . 42

5.1.4 Summary of the parameter variations . . . . . . . . . . . 46

5.2 Advanced Results . . . . . . . . . . . . . . . . . . . 48

5.2 .1 Introduction . . . . . . . . . . . . . . . . 48

5.2 .2 Series $\mathrm{A} 1 \ldots \ldots \ldots \ldots$

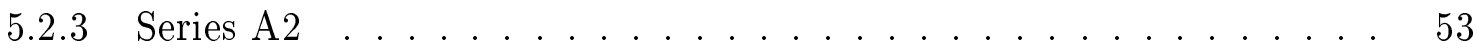

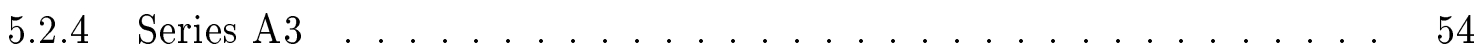

5.3 Summary of the results for bottom heated models . . . . . . . . . . . 55

5.3 .1 Influence of the parameter $a \ldots \ldots . \ldots 55$

5.3 .2 Influence of advection and $m \ldots \ldots$. . . . . . . . . . . . . . . . . .

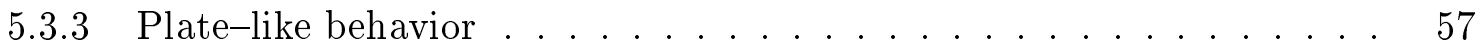

6 Internally heated models I $\quad 59$

6.1 Introduction . . . . . . . . . . . . . . . . . . . 60

6.2 Case ISL $1 \ldots \ldots \ldots \ldots \ldots$. . . . . . . . . . . . . . . . . . . . . . 60

6.2.1 Description of the convection pattern . . . . . . . . . 61

6.2.2 Summary of the observations . . . . . . . . . . . . . . 64

6.2.3 Plate-like behavior, problems and their possible solutions . . . . . 68

6.3 Case ISL $2 \ldots \ldots \ldots \ldots$. . . . . . . . . . . . . . . . . . . . 69

6.4 Case ISL-3 . . . . . . . . . . . . . . . . . . . 74

6.5 Case ISL-4 . . . . . . . . . . . . . . . . . . . 75

6.6 Discussion and Conclusions ...................... 78

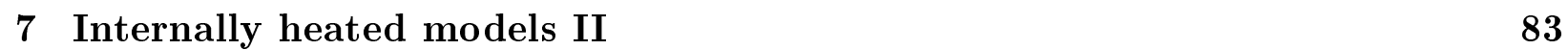

7.1 Introduction . . . . . . . . . . . . . . . . . . 84

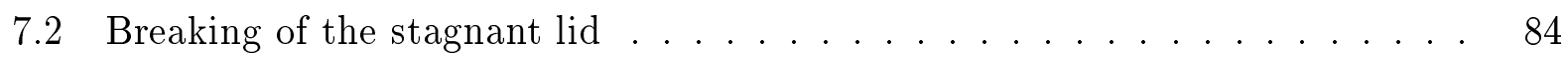

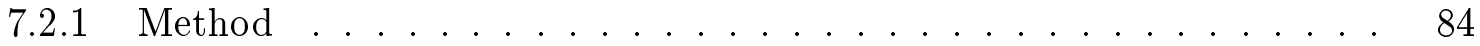

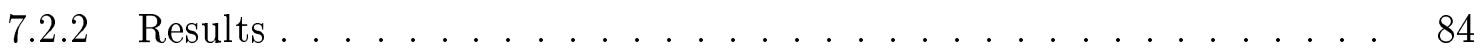

7.2 .3 Comparison with the Earth . . . . . . . . . . 89

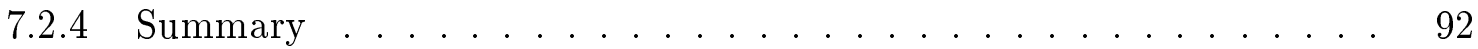

7.3 Temporal evolution of a broken lid . . . . . . . . . . . . . . . 92

7.3.1 Cases IST-1 - IST-3 . . . . . . . . . . . . . . 93

7.3.2 Cases IST-4 and IST-5 . . . . . . . . . . . . . 101

7.3.3 Summary and Discussion . . . . . . . . . . . . . . 102

7.4 Depth-dependent viscosities . . . . . . . . . . . . . 106

7.4 .1 Introduction . . . . . . . . . . . . . . 106

7.4 .2 Cases DD-1 - DD-4 . . . . . . . . . . . . 107

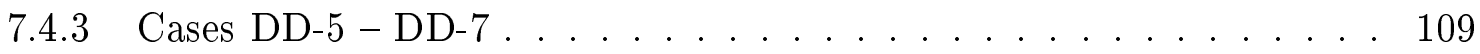

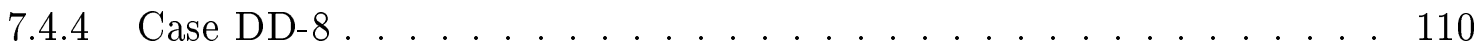




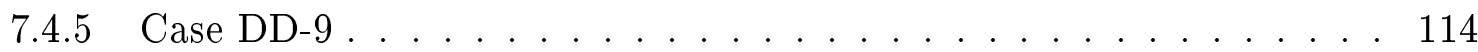

7.4 .6 Summary and Discussion . . . . . . . . . . . . . 114

8 Summary and Discussion $\quad 119$

8.1 Influence of the damage parameters . . . . . . . . . . . . . 120

8.1.1 Convective regimes . . . . . . . . . . . . . . 120

8.1.2 Influence of the damage source parameter $a \ldots \ldots 120$

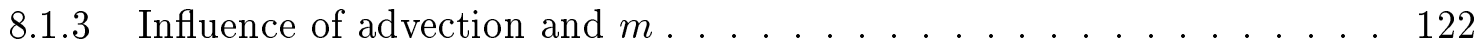

8.2 Plate-like behavior in the simulations . . . . . . . . . . . . . . 122

8.2.1 High viscosity of the surface material . . . . . . . . . . . 123

8.2.2 Localized mid ocean ridges and subduction zones . . . . . . . . . 123

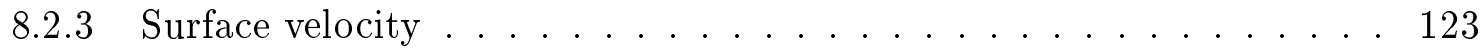

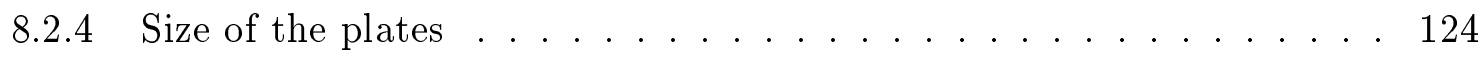

8.2.5 Asymmetry of subduction . . . . . . . . . . . . . 124

8.3 Discussion and Conclusion . . . . . . . . . . . . . . . . . 125

8.3.1 Similarities with previous models ............. 125

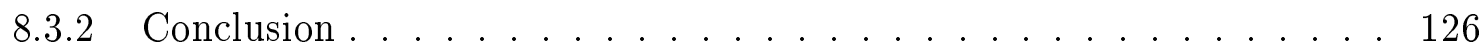

A Appendix $\quad \mathbf{1 2 9}$

A.1 Non dimensional quantities in the Hydrodynamic equations . . . . . . . . 129

$\begin{array}{ll}\text { Literaturverzeichnis } & 132\end{array}$ 


\section{Chapter 1}

\section{Abstract and Outline of the thesis}

\subsection{Abstract}

These thesis investigate a new model to improve plate-like behavior of the lithosphere in two-dimensional mantle convection simulations. Satisfactory plate-like behavior includes localized spreading centers and subduction zones, uniform surface velocities and asymmetric downwellings.

In the new model the common hydrodynamic equations are combined with a viscosity which depends on temperature, depth and an additional damage parameter. The temporal evolution of this parameter is described by a new equation featuring source, sink and advection of damage. It is designed to allow the self-focusing of highly damaged regions especially within the cold lithosphere.

Three different combinations of heating mode, Rayleigh number and temperaturedependence of viscosity are investigated: (a) A bottom heated model with sluggish lid convection (assuming no damage influence), (b) an internally heated model with sluggish lid convection and (c) an internally heated model with stagnant lid convection, which is also the model most comparable to Earth.

It is found that the convective behavior of all simulations can be classified into three regimes: (1) The Low Damage Regime (LDR) shows no significant influence of the damage onto convection. (2) In the Variable Damage Regime (VDR) the damage is strongly time dependent and its variations are correlated with changes in the volume of downwelling material. (3) The Homogeneous Damage Regime (HDR) shows weakly time-dependent intermediate damage values.

Systematical parameter studies show that an increase of the damage source favors regimes with high damage values like the VDR, increases the asymmetry of subduction but weakens and fragmentate plates. A decrease of damage advection focus low viscosity zones (LVZs), allows self lubrication and thus improves the uniform motion of lithospheric material. However, it also promotes strong temporal variations in the convective pattern. Because of these temporal variations the establishment of both, focused LVZs and stable convection in 
the HDR is nearly impossible without an additional depth-dependence of viscosity.

The models most comparable to Earth are those for the parameter combination (c) with depth-dependent viscosity. These models show stable, extended and uniformly moving plates (around $8000 \mathrm{~km}$ length) with focused spreading centers and subduction zones. Although purely one-side subduction of a plate is not obtained the downwelling process is often significantly asymmetric. However, estimates for the damage parameters needed to break a stagnant lid on Earth are at least one order of magnitude too high to be realistic. Nevertheless, the rheological model used in these thesis is an important contribution to the ongoing search for the basic physical mechanisms responsible for Earth's plate tectonics.

\subsection{Outline of the thesis}

To further elucidate the structure of these thesis a short explanation of what is done in the following chapters is given now.

Chapter 2 presents the basic features of Earth's plate tectonics, discusses some previous numerical models with respect to their ability to produce plate-like behavior and finally explains the aim of these thesis.

Chapter 3 introduces the common hydrodynamic equations and the special damagedependent rheology which is used here. Several features of this rheology are also discussed.

Chapter 4 gives a brief overview of the numerical methods used to solve the partial differential model equations. It also discusses numerical and resolution problems.

Chapter 5 is the first of three chapters, which present and discuss results of the numerical simulations. In particular it features models in a box heated from below. Its aim is to understand the influence of the source, the sink and the advection of damage onto the convective structure in the simulation. The bottom heating is chosen, because it favors a high symmetry of convection and is therefore the appropriate model to study symmetry breaks due to the imposed rheology.

Earth is probably $80 \%$ heated from within. Chapter 6 therefore uses internal heating to drive convection. Similar to the previous chapter it investigates the influence of damage on models with otherwise sluggish lid / low viscosity contrast convection. Since no localized upwellings are present in internally heated models the focus here is on the evolution of passive spreading centers.

Solomatov et al. (1995) showed that Earth would not be in the sluggish but in the 
stagnant lid regime, if viscosity would be only temperature dependent. In an internally heated system Chapter 7 therefore investigates

(1) how much damage is at least necessary to break a stagnant lid for variable Rayleigh number and temperature dependence of viscosity,

(2) how variations in the source and the advection of damage influence the temporal evolution of systems with broken stagnant lids and

(3) whether the extremely episodic convective behavior often observed in (2) can be reduced by the introduction of a depth-dependent viscosity.

Chapter 8 summarizes the results obtained for this new rheological model and compares them to previous publications. A final conclusion ends these thesis. 


\section{Chapter 2}

\section{Introduction}

Ever since the relative motion of Earth's plates against each other was discovered scientists tried to find the basic physical mechanisms responsible for this phenomenon. But even today we are still far away from completely understanding plate tectonics. The models and numerical simulations presented in these thesis are a contribution to the ongoing discussion about the reasons for plate tectonics.

This chapter is meant to give a brief introduction into the investigated subject and the underlying motivation:

\subsection{Plate tectonics on Earth}

In this section the basic features of Earth's plate tectonics are presented.

\subsection{Numerical models}

It is explained why computer simulations are performed. Moreover I develop criteria to evaluate plate-like behavior in simulations and discuss whether they are fulfilled in previous simulations.

\subsection{Aim of these thesis}

Based on the results of previous investigations the aim of these thesis is explained. 


\subsection{Plate tectonics on Earth}

In contrast to other planetary bodies like Venus, Mars or the Moon, the Earth's lithosphere is not an immobile, undivided shell, but is broken into several pieces, the so called "plates". Actually, there are seven major and a couple of smaller plates, where the major ones have an extension of several thousand kilometers. These plates are moving relative to each other, forming divergent, convergent and transform boundaries.

\section{- Divergent boundaries}

Two plates move away from each other forming a mid ocean ridge where hot basaltic material is rising from the Earth's mantle. The length of a mid ocean ridge system is in the order of several thousand kilometers, its width amounts to a few kilometers. An example is the Mid Atlantic Ridge (through Island).

\section{- Convergent boundaries}

Two plates are colliding with each other. On convergent boundaries oceanic lithosphere disappears by the subduction of one plate beneath the other. Seismic tomography shows that the "subduction angle" between the subducting plate and the other one is usually around sixty degrees, although higher and lower angles are also observed. Continental lithosphere is never subducted, since it has a much lower density than the mantle. Examples for convergent boundaries are the Tonga trench (oceanic lithosphere subducts beneath oceanic lithosphere), the subduction of the Pacific plate beneath Japan (oceanic lithosphere subducts beneath continental lithosphere) or the Himalaya region (continental lithosphere in collision with continental lithosphere).

\section{- Transform faults}

Two plates slide past each other. The width of transform faults is also in the order of kilometers while their length is thousand or more times higher. The probably most famous example for a transform fault is the San Andreas fault in California.

Typical values for the relative velocity between two plates are in the order of a few centimeters per year. These plate velocities usually remain approximately constant for several millions of years. Through their motion relative to each other the plates are deformed. However, these deformations are limited to quite narrow regions along the plate boundaries.

Plates are continously destroyed (subduction) and rebuilt (mid ocean ridges) and can change their direction of motion. Therefore plate boundaries are not invariable: Entire plates and Mid Ocean Ridges can be subducted (Farallon Plate) or boundaries vanish when two continents collide (Himalaya). New plate boundaries often develop in regions, where the Earth's surface is already weak (see Gurnis et al. 2000). Long living (passive) faults may therefore play an important role in the global structure of plate tectonics.

An important parameter for characterizing plate tectonics is the ratio of kinetic energy in poloidal motion (Mid Ocean Ridges, Subduction) and toroidal motion (transform faults) of 
plates. It is estimated to be around unity by several authors (see Bercovici et al. 2000 and references therein $)^{1}$.

\subsection{Numerical Models}

An important means to understand why the plate tectonics on Earth looks the way it does are numerical simulations. The idea behind these simulations is to develop a model based on general physical laws and special geophysical thoughts. This model is formulated as a system of partial differential equations and solved numerically by computers. If the computed results do not reproduce nature in an appropriate way the model has to be improved.

In the first part of this section I discuss which features a numerical simulation should show to successively reproduce plate tectonics. Later, I present results from previous simulations by other authors.

\subsubsection{Criteria for the evaluation of plate-like behavior in numer- ical simulations}

To evaluate whether a numerical simulation conforms with the above description of plate tectonics and can therefore be judged as "plate-like" I define the following criteria:

- The lithospheric material must have a significant higher viscosity than the mantle material.

- The model has to exhibit very localized plate boundaries (e.g. mid ocean ridges, transform faults and subduction zones).

- The surface velocity of the material must change strongly at plate boundaries but has to remain uniform within the plate.

- The plate velocity has to be in the order of a few centimeters per year and should remain approximately constant for several millions of years.

- Plates must have a size of several thousand kilometers.

- Subduction has to occur asymmetrically. This implies:

1. Different velocities of the two plates at a subduction zone. Without trench imigration $^{2}$ the optimum would be the limit of one mobile and one immobile plate.

\footnotetext{
${ }^{1}$ Although this is an important observation for Earth it will not be explained in more detail, since it is not relevant for the two-dimensional systems investigated here. More details about toroidal and poloidal motion can be found in Busse and Frick (1985)

${ }^{2}$ Trench migration is of course an important feature of plate tectonics since it probably influences the subduction angle. However, since it is not observed in the following calculations and since the trench migration velocity is significantely smaller than the plate velocity this effect is not further regarded here
} 
2. A subduction angle around sixty degrees, where the subduction angle is assumed to be the angle between the already subducted material and the slower of the two plates.

\subsubsection{Review of plate-like behavior in previous numerical models}

Experimental and theoretical investigations show that the stress - strain rate dependence for lithospheric and mantle material on Earth is highly complex. The viscosity $\eta$ is a function of several variables like temperature, pressure, strain rate, grain size, composition etc. ${ }^{3}$ Numerical models to explain plate tectonics on Earth can only succeed, if the most important features of the terrestrial rheology are included. During the last tens of years those models where therefore refined with increasingly complex rheologies. Here I will give a brief overview over the most essential of these refinements. Since this work focuses on the self-consistent evolution of plates, models with artificially introduced weak zones (for example Monnereau and Quere (2001)), prescribed plate velocities (Zhong and Gurnis (1995)) or continental lithosphere ${ }^{4}$ will not be respected in this overview.

\section{Models with temperature- and pressure-dependent viscosity}

Simulations of mantle convection which employ a constant viscosity can not to explain the nearly rigid surface of the lithosphere. Thus, an exponential temperature- and pressuredependence of the viscosity is often assumed

$$
\eta \sim e^{(E+p V) / R T}
$$

where $\eta$ is the viscosity, $E$ the activation energy, $V$ the activation volume, $R$ the gas constant and $T$ the temperature. This rheology law basically reflects the mobility of atoms and dislocations in the crystal lattice (see for example Ranalli 1995). For Earth viscosity variations of about six or more orders of magnitude due to the temperature-dependence are assumed for mantle and lithosphere. Based on geoid and postglacial rebound investigations a pressure-dependent viscosity increase of about two orders of magnitude within the mantle is assumed. However, whether this increase is exponential as in equation 2.1 or more abrupt -for example due to a phase transition- is not exactly known.

Systematic investigations of models with temperature-dependent viscosities have been performed for example by Solomatov (1995) and Ratcliff et al. (1997). Their results can be classified into three regimes depending on Rayleigh number and viscosity contrast: The small viscosity contrast regime, the sluggish lid regime and the stagnant lid regime.

In the stagnant lid regime the uppermost material is very rigid and does not significantly participate in the convective circulation within the box. Downwellings are weak transporting only material from the bottom of the stagnant lid.

\footnotetext{
${ }^{3}$ I can not discuss here in detail how the different quantities which influence the rheology on Earth are identified. However an overview can be found in for example Karato and Wu 1993.

${ }^{4}$ An extended discussion can be found in the AGU monograph 121 "The History and Dynamics of Global Plate Motions" in the articles of Bercovici et al., Tackley and Gurnis et al. (2000).
} 
In contrast the lid of cold and highly viscous fluid in the sluggish lid regime moves along the top surface boundary and is finally subducted.

Convection in the small viscosity contrast regime does not differ very much from iso-viscous convection.

However, none of these regimes shows convincing plate-like behavior. In the stagnant lid regime hardly any material is moving along the surface. The surface velocity in the sluggish lid regime varies more sinusoidal than abrupt and no one-side subduction occurs. The small viscosity contrast regime does not even have a well developed viscosity contrast between lithosphere and mantle.

Solomatov also shows that simulations with Rayleigh numbers and viscosity contrasts which might be realistic for Earth produce always a stagnant lid convection if the viscosity is only temperature-dependent. Therefore, additional mechanisms which prohibit the development of a stagnant lid must play an important role in the Earth.

The influence of a pressure-dependence in the rheology is investigated for example by Bunge et al. (1996) or Christensen and Harder (1991). Bunge concludes that the pressuredependence promotes long scale convective structures which might be relevant for the plate size. For three-dimensional simulations an increase in the toroidal motion is observed by Christensen and Harder. However, a stagnant lid can not be broken with a realistic pressuredependent rheology.

\section{Models with strain-rate-dependent viscosity}

Except for temperature and pressure the viscosity on Earth also depends on the strain rate $(\dot{\varepsilon})$ (see for example Weertman and Weertman (1975) or Evans and Kohlstedt (1995)). This

\begin{tabular}{|c|c|c|}
\hline Case & Range of $n$ & Character of the material \\
\hline 1 & $0<n<1$ & $\begin{array}{l}\text { stress exponentially increases with strain rate } \\
\text { no development of weak zones possible }\end{array}$ \\
\hline 2 & $n=1$ & iso-viscous convection \\
\hline 3 & $1<n<\infty$ & strain rate softening rheology \\
\hline 4 & $n=-\infty$ or $n=\infty$ & $\begin{array}{l}\text { Plastic rheology: } \\
\text { stress constant everywhere in the material }\end{array}$ \\
\hline 5 & $-\infty<n<0$ & $\begin{array}{l}\text { Instable rheology: } \\
\text { stress decreases with increasing strain rate } \\
\text { instable and not realizable }\end{array}$ \\
\hline
\end{tabular}

Table 2.1: Features of the strain-rate-dependent rheology for different exponents $n$. 
dependence can be written in the following form ${ }^{5}$

$$
\eta \sim \dot{\varepsilon}^{-\frac{n-1}{n}}
$$

The stress in the material is therefore $\sigma=\eta \dot{\varepsilon} \sim \dot{\varepsilon}^{1 / n}$ (see table 2.1 and figure 2.1). The size of $n$ determines the character of the material: Since case 1 is clearly not relevant for Earth and the problems with iso-viscous simulations are already explained I now present simulations with rheologies according to the cases 3,4 , and 5 .

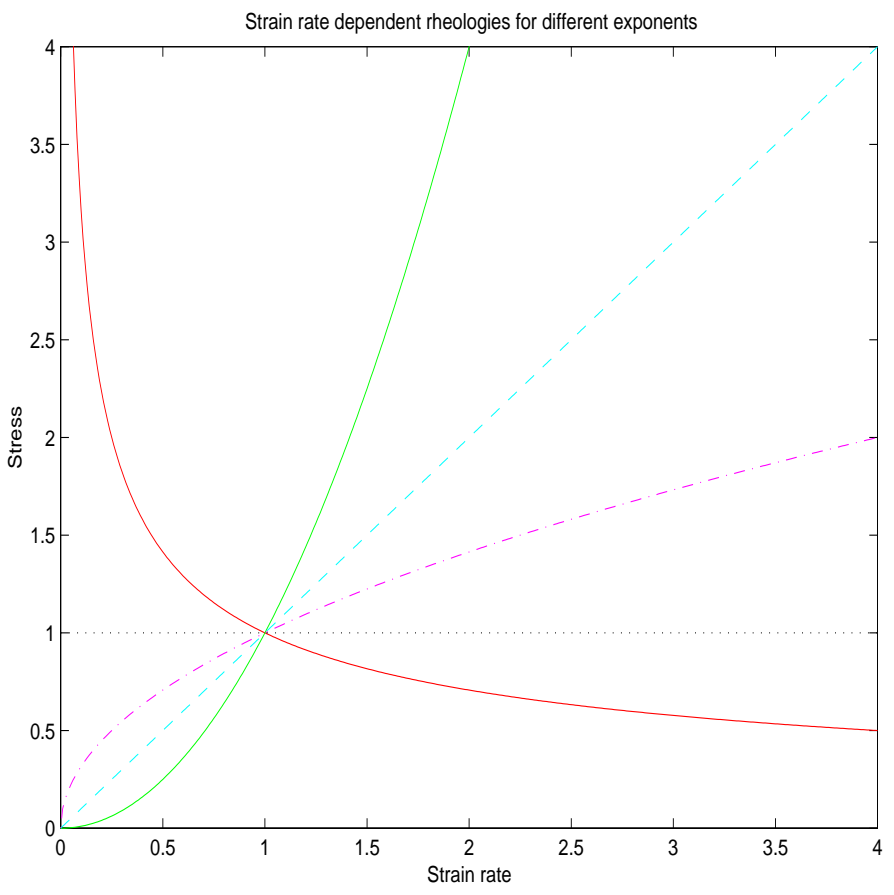

Figure 2.1: Rheology $\sigma \sim \dot{\varepsilon}^{1 / n}$ for $n=1 / 2, n=1, n=2, n=\infty$ and $n=-2$

Strain rate softening rheologies Since experiments (see Evans and Kohlstedt (1995)) suggest that the power law exponent $n$ for Earth might be around 3 a lot of simulations where performed with this kind of viscosity law (Christensen (1984), Christensen and Harder (1991), Weinstein and Olson (1992)). The surface velocities for this rheology differ clearly from a sinus function and change significantly in limited regions for example above downwellings. However, even for unrealistic high power law exponents these regions are much broader than observed on Earth. In addition significant symmetry breaks in the subduction process are not obtained.

\footnotetext{
${ }^{5}$ Of course there are a lot of other formulations for strain-rate-dependent rheologies like the Bingham plastics or bi-viscous laws. However, these are just further mathematical models for essentially the same effects described by this equation.
} 
Yield stress rheologies Yield stress rheologies are a combination between a "regular" viscous and a plastic rheology as in case 4 of the table above. The material behaves viscous until a prescribed yield stress is exceeded, afterwards the stress inside the material remains constant. Although mainly developed and tested for engineering materials (see review of Vermeer 1990) it is thought to be a good description for the semi ductile-semi brittle deformation of the intermediate lithosphere.

I present three investigations with this kind of rheology here in detail. However in a short summary their results about breaking a stagnant lid are:

- For high yield stresses a stagnant lid is not broken.

- For low yield stresses a stagnant lid is broken, but the zones of low viscosities are not very focused and subduction is symmetric.

- For intermediate yield stresses highly episodic behavior is obtained where the lid is broken once in a while, but always regenerates.

Trompert and Hansen (1998) use the harmonic average of $\eta_{T}=e^{-R T}$ and $\eta_{e}=\eta^{*}+\sigma^{*} / \dot{\varepsilon}$ as viscosity law, where $R, \eta^{*}$ and $\sigma^{*}$ are input parameters. They obtain episodical behavior in their three dimensional calculations where large parts of the lithosphere suddenly subduct asymmetric and with homogeneous surface velocity. When the subduction stops no thermal boundary layer is present any more in this region. After the thermal boundary layer is re-grown this cycle starts again. Although the surface velocity is very homogeneous during the fast subduction episodes and the subduction is clearly asymmetric this kind of episodic subduction behavior appears more Venus- than Earth-like. Moreover no transform faults can be observed in this calculation.

Additional introduction of depth-dependence for the viscosity inhibits the very episodic behavior as shown by Stein (2000).

Moresi and Solomatov (1998) present more systematical two dimensional studies with $\eta=\eta_{\text {creep }}=e^{-\gamma T}$ for $\sigma_{\text {creep }}<\sigma_{\text {yield }}=\left(\sigma_{0}+\sigma_{1} z\right)$ and $\eta=\eta_{\text {yield }}=\left(\sigma_{0}+\sigma_{1} z\right) / \dot{\varepsilon}$ for $\sigma_{\text {creep }} \geq \sigma_{\text {yield }}\left(\sigma_{0}\right.$ and $\sigma_{1}$ are input parameters $)$. The yield stress is depth-dependent here to better include the brittle behavior of the uppermost lithosphere $(0-20 \mathrm{~km})$ and the semi-brittle, semi-ductile behavior of the intermediate lithosphere (20-40 km) into the model (see Kohlstedt et al. 1995). Moresi and Solomatov considered models, which would produce stagnant lid convection for simply temperature-dependent rheology and conclude that three different convective regimes can be distinguished, depending on the size of the yield stress: For high yield stresses the stagnant lid is not significantly changed. For low yield stresses a mobile lid is obtained which moves with a quite uniform velocity some distance away from the box boundaries. Between these two regimes episodic behavior with temporal transitions between the two other regimes is observed.

Tackley (2000b and 2000c) uses a very similar viscosity law for his calculations: $\eta=\min \left(\eta(z, T), \sigma_{y}(z) / 2 \dot{\varepsilon}\right)$ with $\sigma_{y}(z)=\min \left((1-z) \sigma_{y \text {-brittle }}, \sigma_{y \text {-ductile }}\right)$. Tackley concludes, that first order plate-like behavior can be obtained with this kind of rheology 
only "in a narrow range of yield strength, below which diffuse boundaries, and above which episodic behavior and eventually a rigid lid are observed". However, asymmetrical subduction and the evolution of pure transform faults are not observed in these calculations. The additional introduction of an asthenosphere by reduction of the viscosity by a factor of ten if a melting temperature is exceeded (Tackley 2000c) improves the results with respect to piecewise uniform homogeneous surface velocities and the range of yield stresses for which this first-order plate-like behavior can be obtained. The asymmetry of subduction or the evolution of transform faults is not significantly improved by the introduction of the asthenosphere. In contrast the effect of an exponential increase of the viscosity with depth by a factor of ten is much smaller then the effect of the asthenosphere.

Self-lubricating rheologies Similar to the yield stress rheologies these viscosity laws are combinations between a viscous and an instable viscosity law. For low strain rates the material behaves viscoelastic but once a limit value $\left(\dot{\varepsilon}_{\max }\right)$ is exceeded stress starts to decrease with increasing strain rate. In consequence the material develops infinite narrow shear zones with zero viscosities.

As far as I know the only attempt to use a self-lubricating rheology in a numerical model is made by Tackley (1998). Basically his idea is to compensate the instable rheology of a one grid-point thick self-lubricating lithosphere with

$$
\eta=\frac{10^{4} \cdot \sigma_{\max }^{2}}{\sigma_{\max }^{2}+10^{8} \cdot \dot{\varepsilon}^{2}}
$$

( $\sigma_{\max }$ is an input parameter) by an iso-viscous convecting mantle. A temporal evolution of this model is not calculated, just the viscosity and flow fields for temperatures obtained from iso-viscous simulations. However, the results are extremely uniform surface velocities and sharp plate boundaries.

\section{Models with damage-dependent rheologies}

While searching for other quantities which might influence Earth's rheology it was found that mantle and crustal ductile shear zones are often characterized by very small grains, hydration and elevated temperatures (see Vissers et al. (1995), Pili et al. (1997) and Jin et al. (1998)). How exactly grain size or water influence the viscosity is however poorly understood. Some scientists therefore constructed rheology laws, which are a plausible formulation for all three of these mechanisms. The new parameter in these rheologies was usually termed the "damage" parameter (Tackley 2000c).

However, the important new aspects in the construction of the damage-dependent rheologies are their abilities to produce self-lubricating low viscosity zones (LVZs) ${ }^{6}$ and long living

\footnotetext{
${ }^{6}$ To avoid misunderstandings: The term "low viscosity zone" is used for an area with a significant influence of the damage onto viscosity. The existence of an asthenosphere which is frequently described with the same
} 
fault systems.

The first results with damage-dependent-rheologies are presented by Bercovici $(1996,1998)$ who investigates the evolution of transform faults in a two dimensional horizontal plate, including a "void-volatile" mechanism into his model. He associates the damage parameter $d$ with the porosity of an iso-viscous matrix material. The pores in the matrix are assumed to be filled with a fluid of lower but also constant viscosity. The corresponding viscosity law is

$$
\eta=\eta_{m}(1-\lambda d)
$$

with $\lambda=\left(\eta_{m}-\eta_{v}\right) / \eta_{m} . \eta_{m}$ is the matrix viscosity and $\eta_{v}$ the viscosity of the fluid. The damage parameter $d$ varies in time according to

$$
\frac{\partial d}{\partial t}+u_{h} \cdot \underline{\nabla}_{h} d=a \frac{\sigma^{2}}{4(1-d)}-b d+\beta \nabla_{h}^{2} d
$$

with $u_{h}$ the horizontal velocity, $\sigma$ the stress $\beta$ the chemical diffusivity and $a$ and $b$ parameters. Except for the last term on the right hand side (horizontal diffusion) the other terms are very similar to what is used in my rheology and will be motivated later (see section 3.2). Bercovici concludes that for "relatively inviscid fluid a state of highly plate-like motion (i.e. with uniform strong "plate" interiors, weak margins and extremely focused strike-slip shear zone)" is obtained. Moreover his plate boundaries are long lived and survive even while inactive.

Tackley (2000c) combined his yield stress rheology with equations 2.4 and 2.5 and concludes from three dimensional calculations that the adding of damage "(1) improves localization at spreading centers but leads to an increasingly complex network of spreading centers fragmenting the plates, (2) weakens convergent boundary zones and can make downwellings highly episodic and (3) does not lead to pure transform boundaries in these calculations". He also observes, that "single-sided (asymmetric) subduction is common in some of the cases with strain weakening" (that means damage-dependent rheology). However, Tackley performs only a few calculations with damage-dependent viscosity. A systematical study of the influence of the different input parameters in the model is not presented.

\subsection{Aim of these thesis}

The results I presented in the last section clearly demonstrate, that the fundamental physical reasons for plate tectonics on Earth are not completely understood yet. Even the most recent models with yield stress or damage-dependent viscosities have some problems in reproducing plate-like behavior.

term by other authors is not implied here. 
However, in my opinion damage-dependent rheologies show the best potential to gain more insight into the generation of plate-like behavior in mantle convection simulations, because they are able to ......

- ......show self-lubricating behavior, if they are constructed in an appropriate way. This self-lubrication probably allows the evolution of narrow transform faults (Bercovici 1998)), strongly localized divergent boundary zones (Tackley (2000c)) and uniform surface velocities in large parts of the computational surface.

- .......produce asymmetric subduction (Tackley (2000c)).

- ......create long living passive faults (Bercovici 1998).

Despite of these advantages extended studies of damage-dependent rheologies are never done before. The aim of these thesis is therefore to systematically investigate the possibilities of a special damage-dependent rheology to improve plate-like behavior in numerical simulations of mantle convection.

Since a systematical parameter investigation of a three-dimensional model is beyond the available computational capabilities I am limited to two-dimension calculations. This restriction prohibits the study of transform faults. Instead I focus on the generation and development of low viscosity zones (LVZs), piecewise uniform surface velocities and asymmetric subduction to classify plate-like behavior in my simulations. 


\section{Chapter 3}

\section{The Model}

In this chapter I present the equations describing my model for mantle convection, explain the underlying physical ideas and discuss some important features of the model rheology.

\subsection{Hydrodynamic equations}

These equations (sometimes with small modifications) are the basis for most of the published mantle convection models.

\subsection{Rheology equations}

The basic difference between various mantle convection models is usually the kind of rheology included. Here, I present my viscosity and damage equation and discuss their physical significance.

\subsection{Discussion of the Rheology Equations}

The most important features of the used rheology are shown including

(1) the ability to produce self-lubricating LVZs,

(2) the influence of damage-advection and

(3) a simple example. 


\subsection{Hydrodynamic Equations}

My model is basically described by the common equations of mass, momentum and energy conservation in Boussinesq approximation and with infinite Prandtl number. For a twodimensional rectangular domain with one horizontal $(\mathrm{x})$ and one vertical ( $\mathrm{z}$ ) direction these equations can be written as follows:

$$
\begin{array}{lr}
\nabla \cdot \underline{u}=0 & \text { Continuity } \\
-\underline{\nabla} p+\nabla \cdot(\eta \underline{\underline{\varepsilon}})+R a T \underline{e}_{z}=0 & \text { Stokes } \\
\frac{\partial T}{\partial t}+\underline{u} \cdot \underline{\nabla} T=\nabla^{2} T+q & \text { Energy }
\end{array}
$$

Where $\underline{u}=\left(u_{x}, u_{z}\right)$ is the velocity vector, $p$ the non-hydrostatic pressure, $T$ the temperature, $t$ the time, $\eta$ the viscosity and $\underline{\underline{\dot{\varepsilon}}}$ the strain rate tensor defined as

$$
\varepsilon_{i j}=\frac{\partial u_{i}}{\partial x_{j}}+\frac{\partial u_{j}}{\partial x_{i}} .
$$

Finally, $q$ is the rate of internal heating, being zero for bottom heated models and one for internally heated.

All equations are already written in a non-dimensional form for which $x=\frac{\tilde{x}}{h}$, $(z$ analous $)$, $t=\frac{\kappa_{o}}{h^{2}} \tilde{t}, T=\frac{\tilde{T}-\tilde{T}_{o}}{\tilde{T}_{u}-\tilde{T}_{o}}, p=\frac{\left(\tilde{p}-g \rho_{0} z\right) h^{2}}{\kappa_{0} \eta_{0}}, \eta=\frac{\tilde{\eta}}{\eta_{0}}, \kappa=\frac{\tilde{\kappa}}{\kappa_{0}} \rho=\frac{\tilde{\rho}}{\rho_{0}}$ and $q=\frac{\tilde{q}}{q_{0}}$ and (quantities with the symbol still have a dimension) were used as discussed in Christensen and Harder (1991). The corresponding Rayleigh number for bottom heated models is therefore defined as

$$
R a_{b h}=\frac{\rho_{0} \alpha g \Delta T h^{3}}{\eta_{0} \kappa_{0}}
$$

and for an internally heated models as

$$
R a_{i h}=\frac{\alpha g q_{0} h^{5}}{\eta_{0} \kappa_{0}^{2} c_{p}},
$$

where $\rho_{0}$ is the density of the material at the surface, $\alpha$ the thermal expansivity, $g$ the gravitational acceleration, $\Delta T$ the temperature drop over the box, $h$ the height of the box, $\eta_{0}$ the reference viscosity at $T=0$ and $d=1$ (where $d$ is the damage parameter determinated by equation 3.8$), \kappa_{0}$ the thermal diffusivity, $q_{0}$ the volumetric rate of internal heating and $c_{p}$ the heat capacity.

Typical values for these quantities on Earth are given in the Appendix. However, two important scaling relations should be mentioned here: For $\kappa_{o}=10^{-6} \mathrm{~m}^{2} / \mathrm{s}$ and $h=3 \cdot 10^{6} \mathrm{~m}$ a non-dimensional velocity of 1000 corresponds to a dimensional velocity of $1.05 \mathrm{~cm} / \mathrm{yr}$ and a non-dimensional time of 0.01 (scaled with the thermal diffusion time) corresponds to $2.85 \cdot 10^{9}$ years, roughly half the age of the Earth. 
The boundary conditions on the side walls of the computational domain are periodic and the upper and lower boundaries are assumed as free slip. The system is isothermal $(T(x, z=1)=0)$ at the upper boundary; at the bottom boundary it is either isothermal $(T(x, z=0)=1)$ in the case of bottom heating or uses $\partial T / \partial z=0$ in case of internal heating.

\subsection{Rheology Equations}

The viscosity used in this model is a special representation of the damage-dependent rheologies I presented in the last chapter (see subsection 2.2.2):

$$
\eta=\frac{2}{1+d^{m}} e^{-\gamma T+\zeta(1-z)}
$$

Viscosity

where $m, \gamma$ and $\zeta$ are controlling parameters of the model. If not stated otherwise, $\zeta=0$ is assumed in my models. The damage parameter $d$ is assumed to be time-dependent according to:

$$
\frac{\partial d}{\partial t}+\underline{u} \cdot \underline{\nabla} d=a \sigma \dot{\varepsilon}-b e^{\gamma T} d
$$

Damage

Here $\dot{\varepsilon}$ and $\sigma$ denote the second invariants of the strain-rate and stress tensors, defined e.g. for the stress tensor as

$$
\sigma=\left(\frac{1}{2} \sum_{i, j} \sigma_{i j}^{2}\right)^{1 / 2}
$$

The boundary conditions for the damage parameter are periodic at the side walls and $\partial d / \partial z=0$ is assumed at the top and bottom boundaries.

The two rheology equations 3.7 and $3.8^{1}$ are developed associating the dimensional damage parameter $\tilde{d}^{2}$ with the inverse grain size of the convecting material. A typical value is $\tilde{d}_{0}=\left(10^{-3} \mathrm{~m}\right)^{-1}$. The viscosity equation 3.7 represents the harmonic average between the pure temperature-dependent viscosity $\eta_{T}=e^{-\gamma T}$ and the grain size sensitive viscosity (diffusion creep) $\eta_{d}=d^{-m} e^{-\gamma T}$.

The source term $a \sigma \dot{\varepsilon}$ in equation 3.8 represents grain size reduction due to dynamical recrystallization. Since $\tilde{\sigma} \tilde{\dot{\varepsilon}}$ is the dissipation per unit-volume available in the system, $\tilde{a}$ has the dimension of an inverse surface energy per area. The form of the sink term is taken from investigations of Karato (1984) on grain growth except it assumes linear $d$ dependence. The dimensional sink term parameter $\tilde{b}$ is the decay rate of $\tilde{d}$ at surface temperature.

\footnotetext{
${ }^{1}$ Since the damage parameter influences the convection only via the viscosity I summarize both the viscosity and the damage equation under the name "rheology equations", although this might not be the common nomenclature.

${ }^{2}$ Quantities with the $\sim$ sign are dimensional.
} 


\subsection{Discussion of the Rheology Equations}

Damage-dependent rheologies which are constructed in an appropriate way have two important advantages compared to many other rheological models:

(1) The mechanism of viscosity reduction does not only depend on instantaneous quantities like the strain rate. This encourages the development of long living passive weak zones.

(2) Damage-dependent rheologies allow the self-focusing evolution of very narrow low viscosity zones.

\subsubsection{Self-lubrication neglecting the advection of damage}

I demonstrate now that the proposed rheology is capable of self-lubricating behavior and the formation of focused zones of high strain and low viscosity. This part follows calculations done for similar rheologies by Bercovici(1996, 1998) and Tackley(1998).

The basic idea here is to derive an approximation for the stress-strain-rate-dependence $(\sigma(\dot{\varepsilon}))$ using equations 3.7 and 3.8 and assuming (a) that the convective system reaches a steady state and that (b) the advection of damage is negligible, i.e. the left side of equation 3.8 vanishes. Thus

$$
d\left(1+d^{m}\right)=2 \frac{a}{b} \eta_{T}^{2} \dot{\varepsilon}^{2}
$$

is obtained. An analytical expression for $d$ is possible only for special values of $m$. However, using $d>>1^{3}$ leads to

$$
d \approx\left(2 \frac{a}{b} \eta_{T}^{2} \dot{\varepsilon}^{2}\right)^{1 /(m+1)}
$$

and using equation 3.7

$$
\sigma(\dot{\varepsilon})=\frac{2 \eta_{T} \dot{\varepsilon}}{1+\left(2 \frac{a}{b} \eta_{T}^{2} \dot{\varepsilon}^{2}\right)^{m /(m+1)}}
$$

The top frame in figure 3.1 shows this stress- strain-rate-dependence for fixed values of the source $(a)$ and sink $(b)$ of damage obtained numerically without the assumption $d>>1$ and for $\gamma=0$. For $m<1$ the stress monotonically increases with increasing strain rate as for the "typical" strain rate weakening power law rheologies (see sub-subsection 2.2.2).

$m=1$ represents yield stress rheologies, with the stress becoming constant in the limit of infinite strain rate (see also equation 3.11 ).

For $m>1$ the stress increases with increasing strain rate till a maximum value $\sigma_{\max }$ is

\footnotetext{
${ }^{3}$ Despite this assumption, I will use $\eta=\left(1+d^{m}\right)^{-1} \eta_{T}$ later. This method produces good $\sigma(\dot{\varepsilon})$ approximations for $\dot{\varepsilon}<<1$ and $\dot{\varepsilon}>>1$ and reasonable results for intermediate strain rates (see figure 3.2 )
} 
reached and decreases thereafter. Self-lubrication is initiated when $\dot{\varepsilon}$ exceeds $\dot{\varepsilon}_{\text {max }}$. From equation 3.11 the following approximations are easily derived:

$$
\dot{\varepsilon}_{\text {max }}=\left(\frac{b}{2 a}\right)^{1 / 2}\left(\frac{1}{\eta_{T}}\right)\left(\frac{m+1}{m-1}\right)^{(m+1) / 2 m}
$$
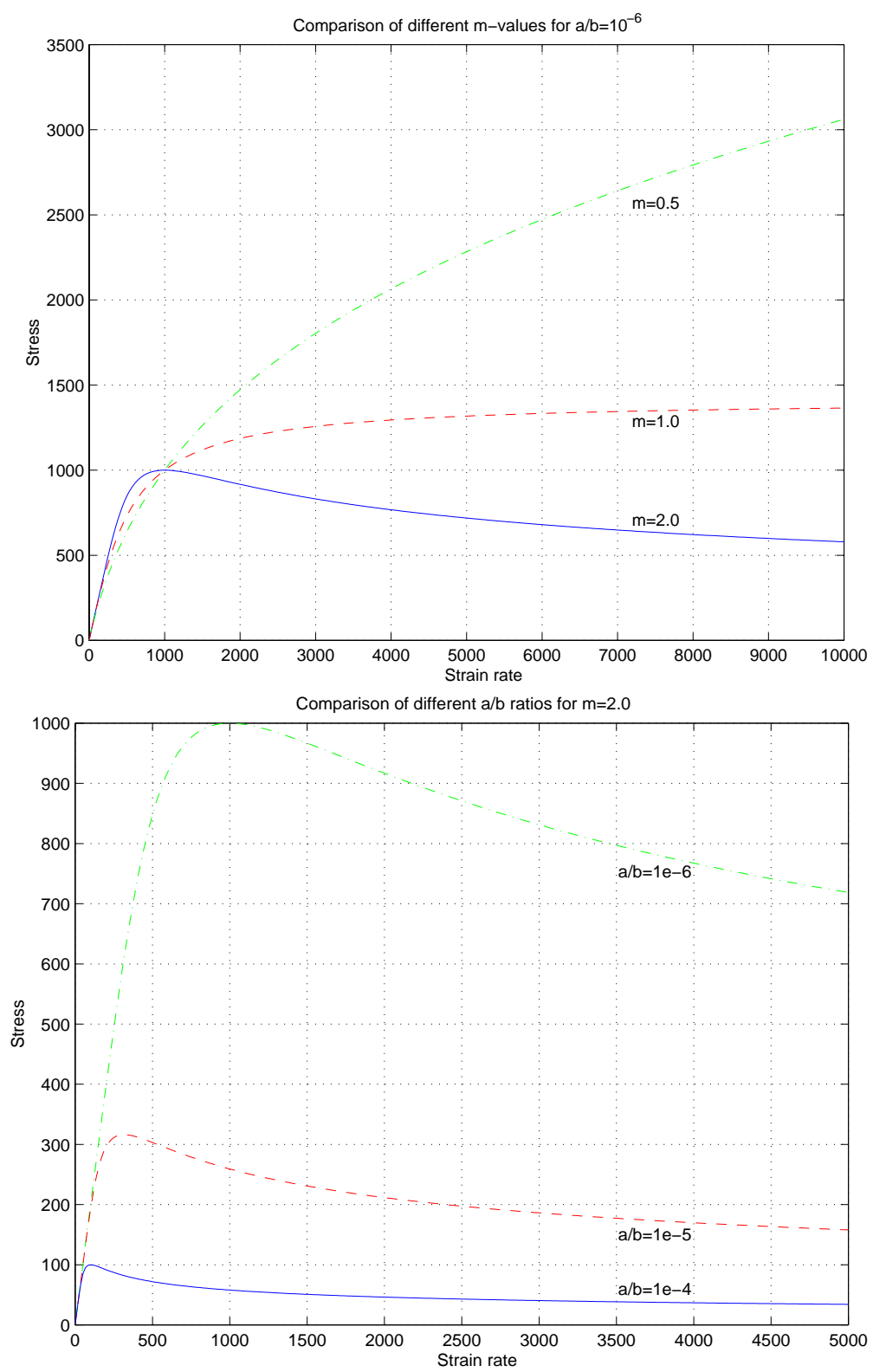

Figure 3.1: Stress-strain rate relations in the limit of a vanishing advection term in the damage equation. Top frame: $\sigma(\dot{\varepsilon})$ for $m=0.5, m=1.0$ and $m=2.0$ with $a / b=10^{-6}$ fixed. Bottom frame: $\sigma(\dot{\varepsilon})$ for $m=2.0$ fixed and $a / b=10^{-4}, a / b=10^{-5}, a / b=10^{-6}$ 


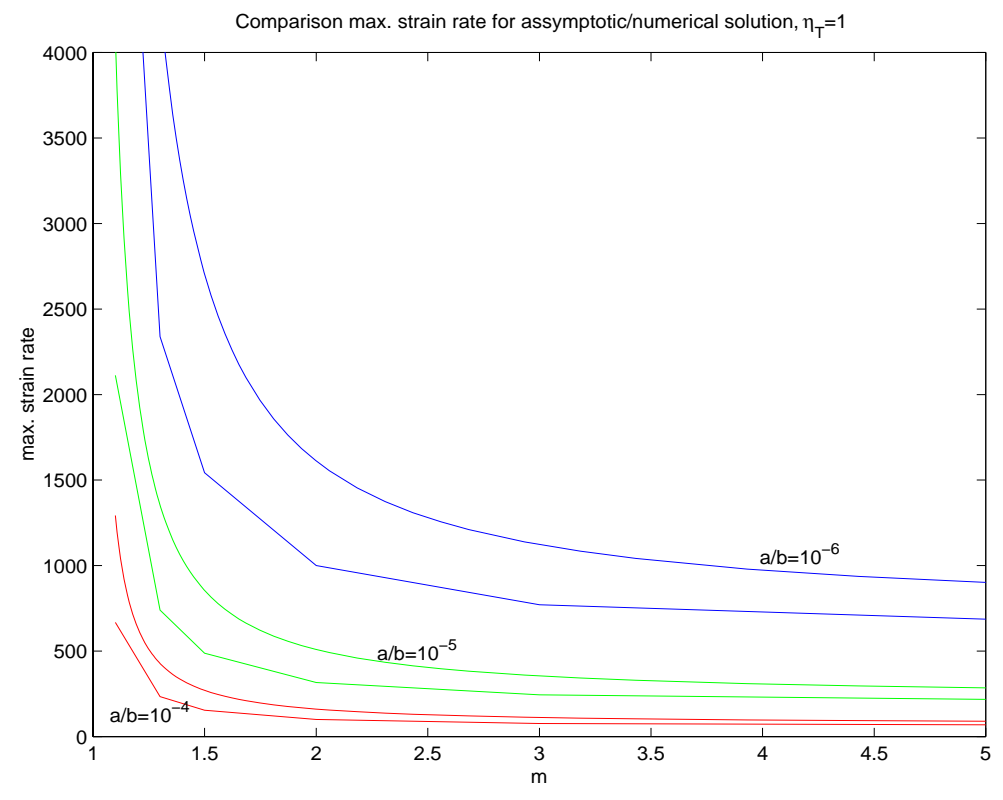

Figure 3.2: The strain rate $\dot{\varepsilon}_{\text {max }}$ for which the stress maximum $\sigma_{\text {max }}$ is reached versus $m$ for the proposed rheology in the limit of vanishing damage advection and for three values of $a / b$. The smooth curves are approximations obtained from equation 3.12, the others represent the numerical solutions for selected $m$ 's.

and

$$
\sigma_{\text {max }}=\left(\frac{2 b}{a}\right)^{1 / 2}\left(\frac{m-1}{2 m}\right)\left(\frac{m+1}{m-1}\right)^{(m+1) / 2 m}
$$

They show that both $\sigma_{\max }$ and $\dot{\varepsilon}_{\max }$ decrease with increasing $a / b$ and increasing $m$ (figures 3.1 and 3.2). This result is quite intuitive: For $m>1$ self-lubricating behavior can be obtained more easily if (a) more damage is produced ( $a$ higher) or if (b) the viscosity is stronger influenced by the damage ( $m$ higher).

Another important aspect in this rheology is the temperature-dependence of viscosity and damage: In the viscosity equation (3.7) the temperature-dependence is responsible for the high viscosity of the cold lithosphere. Its role in the damage equation 3.8 is to prevent the mantle from being heavily weaken by the damage introduced to break the lithosphere.

\subsubsection{Influence of the advection of damage}

In all of the simulations I will present in the following chapters the advection of damage has a destructive effect on low viscosity zones (LVZ). To illustrate this, I prescribe a special 2D velocity field, neglect the temperature-dependence of damage and viscosity and calculate a steady state solution for the damage parameter from the simplified version of equation 3.8 for different values of $a$ and $b$. Comparing these results will help to better understand the 
influence of damage advection.

The horizontal velocity component $u_{x}$ is calculated according to

$$
u_{x}=\frac{1000 c}{1+e^{c(x-z) / 0.01}}
$$

where $c=1$. By using the same formula but with $c=-1$ for the vertical velocity $u_{z}$ one gets the complete velocity field shown in figure 3.3. The change in the direction of the flow causes a diagonal high strain rate zone in the box. It is important, that the material flows obliquely to this high strain rate zone and that damage can therefore be advected away from its source region. In situations when neither a flow oblique to an extended high strain rate zone nor a strain rate variation along that zone is present (transform faults or my example using $u_{z}=u_{x}$ ), the advection term in equation 3.8 vanishes.

The second picture in figure 3.3 shows the steady state viscosity at a depth $z=0.5$ for $a=1.0, b=50000 ; a=0.1, b=5000$ and $a=0.01, b=500$. On the box side where the material flows in, the viscosity is the same in all cases and no influence of the damage parameter is visible. For high $a$ and $b$ values the LVZ is quite sharp, nearly symmetric and the lowest viscosity value is around $\eta_{\text {min }}=10^{-2.2}$. The asymmetry of the LVZ increases with decreasing $a$ and $b$, in particular the slope of the downstream side decreases, the LVZ becomes wider, $\sigma_{\min }$ increases and the position of $\sigma_{\min }$ is advected away from the position of the maximal strain rate. The damage parameter is clearly transported with the material. Since material movement oblique to the LVZs is a frequent situation in my simulations the advection term is always relevant for me. Thus the ratio $a / b$ is not the only determining factor in the following results (like in equation 3.12) but the absolute values of $a$ and $b$ influence the sharpness of LVZs and $\eta_{\text {min }}$.

\subsubsection{Influence of the damage on the velocity: A simple example}

In the previous two subsections I simplified my model by eliminating the coupling between the Stokes and the damage equation. Thus it was possible to demonstrate the self-lubricating potential of the proposed rheology and the influence of damage advection on viscosity structures. However, the analysis of a coupled system finally requires the solution of the entire set of the governing equations. A simple example showing the evolution of a LVZ is therefore presented here.

The following model parameters are chosen: aspect ratio $2, R a=10^{4}, \gamma=\ln \left(10^{3}\right), a=0.04$, $b=10^{4}, m=2$ and bottom heating. The initial condition for temperature is obtained from the corresponding iso-viscous calculation and $d \equiv 1$ is initially assumed for the damage.

Figure 3.4 shows the viscosity fields at the beginning of the calculation, after a short time and at the final steady state. Also presented are the horizontal velocity fields corresponding to the first two viscosities. Finally the horizontal surface velocity for all the cases is shown. It can be seen, that a "v"-shaped LVZ ("conjugate shear zones" in geological terms) devolves above the downwelling, while the velocity field tends to become a "corner-flow" similar to the prescribed field in the last section. A broad zone of viscosity reduction can also be seen 

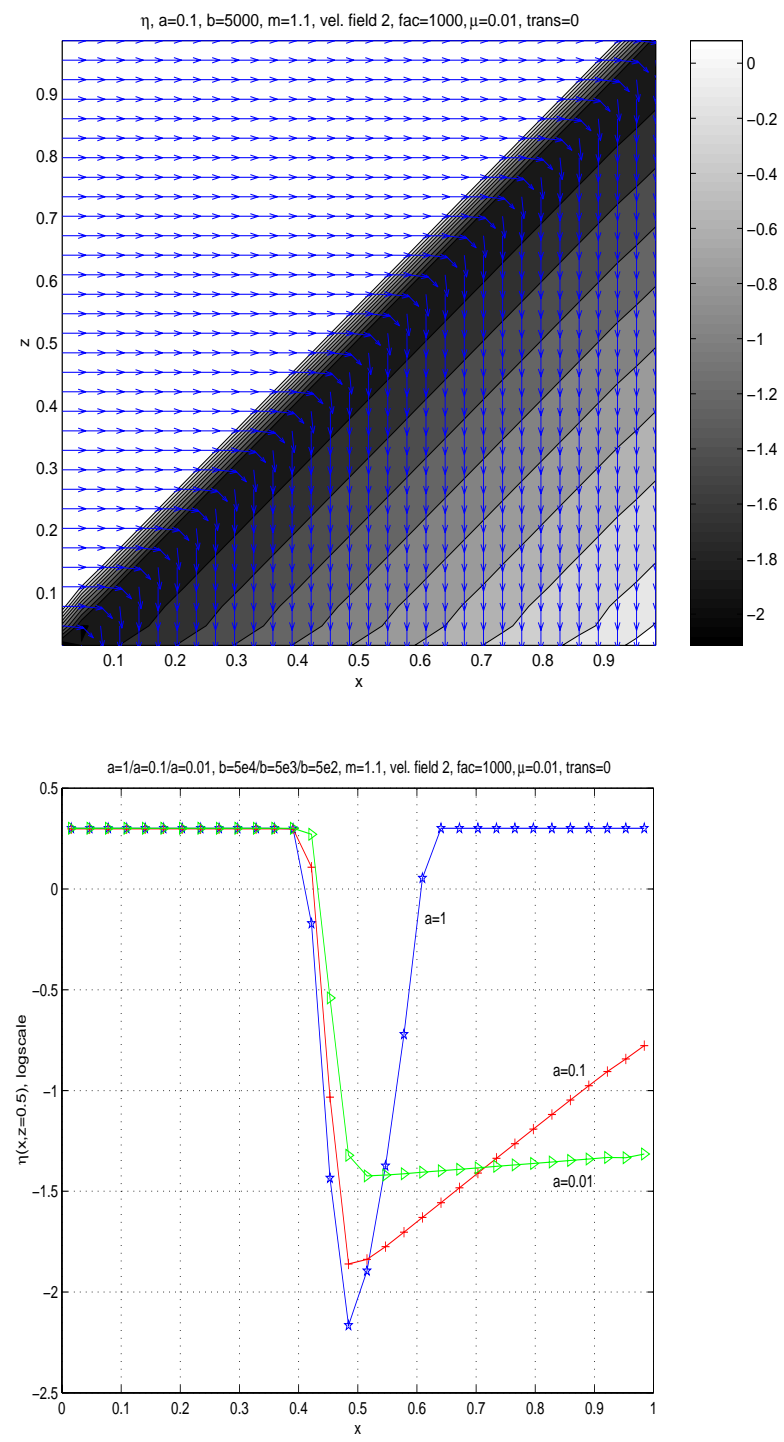

Figure 3.3: Top frame: Viscosity (gray isolines, $a=0.1, b=5 \cdot 10^{4}$ ) and prescribed velocity field (arrows) for investigating the influence of damage advection. Bottom frame: Viscosity at depth $z=0.5$ for three different sets of $a$ and $b$.

above the upwelling, however, the viscosity inside is much higher than in the LVZ above the downwelling. The surface velocity between these two LVZs tends to become increasingly constant and plate-like. Although the shape and sharpness of the low viscosity zones and their minimum viscosity depend on the values of $m$ and $a$ (with $a / b$ constant) and the asymmetry of the system depends on $a / b$ (with $b$ constant), these features are typical for a calculation with significant damage. 


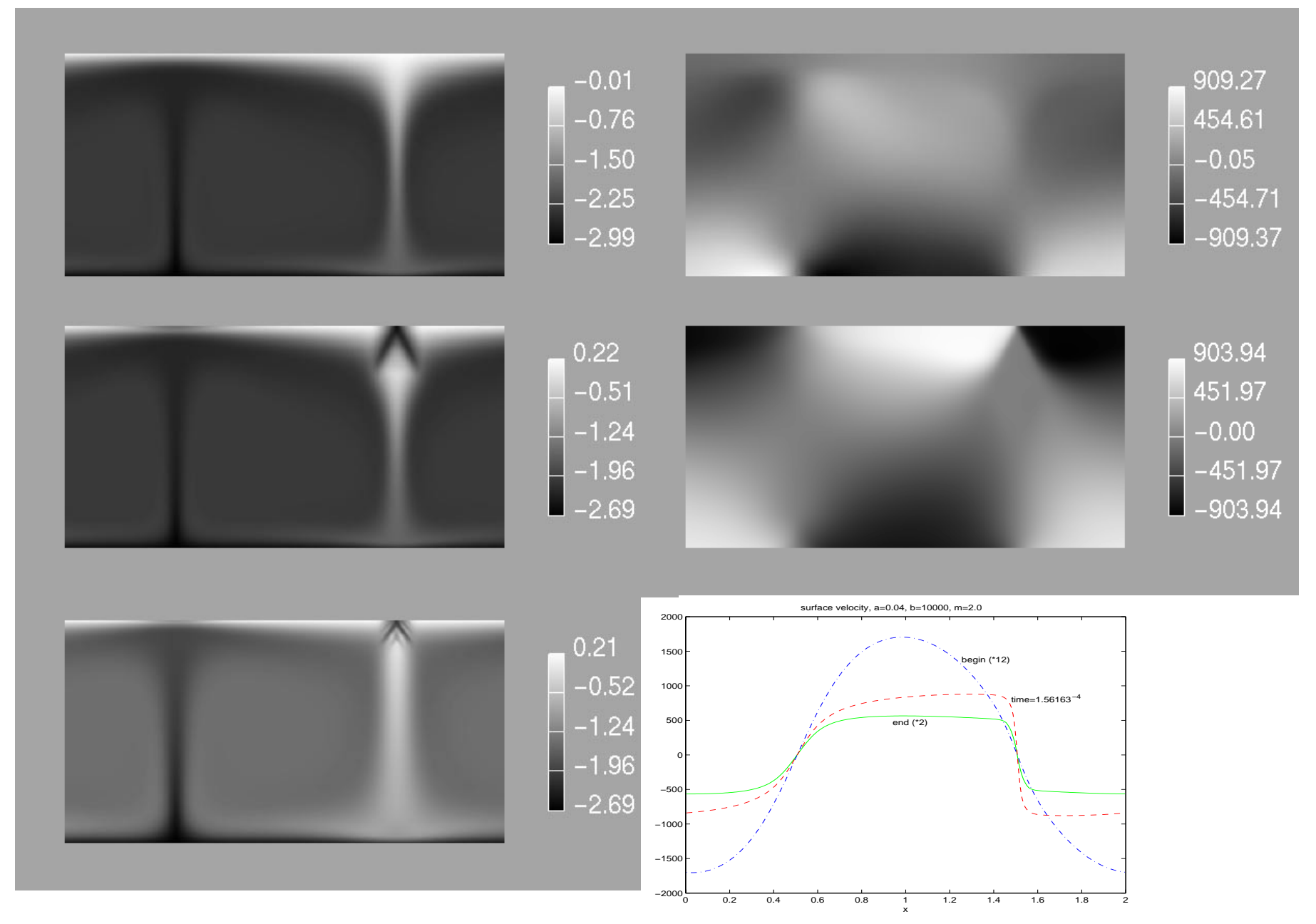

Figure 3.4: Example for the influence of damage. First row: Viscosity and horizontal velocity for a calculation with pure temperature dependent viscosity $\left(R a=10^{4}, \gamma=\ln 10^{3}\right)$. The corresponding temperature field is used as initial condition for a calculation with damage dependent rheology ( $a=$ $\left.0.04, b=10^{4}, m=2.0, R a=10^{4}, \gamma=\ln 10^{3}, d_{i n i t} \equiv 1.0\right)$. Second row: Viscosity and horizontal velocity for the damage dependent rheology at time $=1.56163 \cdot 10^{-4}$. Third row left: Steady state viscosity field for the damage dependent rheology. Right: Surface velocity corresponding to the three viscosity fields. 


\section{Chapter 4}

\section{The numerical treatment of the model equations}

This chapter basically describes the numerical treatment of the equations presented in the last chapter.

\subsection{Numerical Methods}

The discretization of the Model Equations and the algorithms for their solution are explained.

\subsection{Test of the Algorithm}

The results of the computational code are verified.

\subsection{Numerical Limitations}

Shows how different numerical problems are treated here.

\subsection{Resolution of Low Viscosity Zones}

Discusses the number of grid-points required to resolve a low viscosity zone (LVZ) and the effects of under-resolution of a LVZ.

\subsection{Controlling Parameters}

Presents some parameters used to evaluate my results. 


\subsection{Numerical methods}

The numerical methods used to solve the model equations are described in detail in my Diplom thesis (Auth, 1997) and in the article by Auth and Harder (1999). Therefore, only a brief summary is given here.

\subsubsection{Discretization}

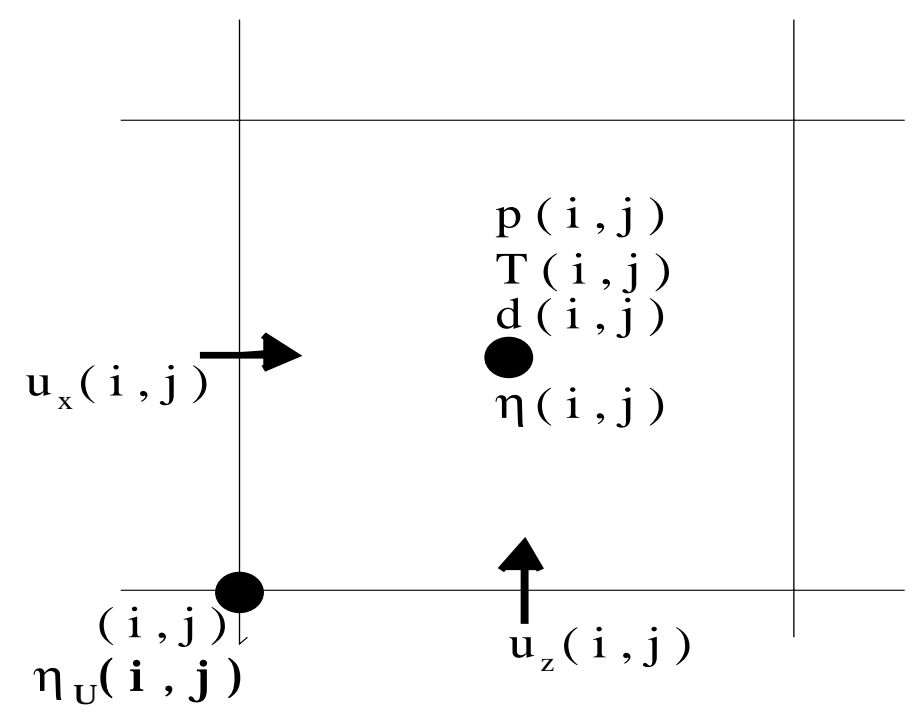

Figure 4.1: Positions of the variables in a grid cell $\Omega_{i j}$

For the discretization of the model equations in the two-dimensional rectangular domain a uniform staggered grid is used (see figure 4.1). The Continuity and the Stokes equation 3.1 and 3.2 are discretized in a straight forward way, using a second order finite volume method for the spatial derivations.

The temporal discretization of the temperature and damage equations 3.3 and 3.8 is performed with the Crank-Nicolson method, however, the velocities in the advection term are assumed to be constant ${ }^{1}$.

For the treatment of the advection terms the upwind scheme suggested by Koren (1993) is used. This method is chosen, because it is simple and produces acceptable results. Since it differs from the scheme used in my Diplom thesis I briefly want to describe it here:

Using $\nabla \cdot \underline{u}=0$ the advection term can be written as:

$$
\begin{aligned}
\underline{u} \cdot \underline{\nabla} T & =\nabla \cdot(\underline{u} \cdot T) \\
& =\frac{\partial\left(u_{x} \cdot T\right)}{\partial x}+\frac{\partial\left(u_{z} \cdot T\right)}{\partial z} .
\end{aligned}
$$

\footnotetext{
${ }^{1}$ With this approach, the Crank-Nicholson method is not of second order any more. However, it allows to keep the numerical treatment of the Continuity and Stokes equation on the one hand and Temperature and Damage equation on the other hand strictly separated
} 
To determine the divergence in the middle of the grid cell $\Omega(i, j)$ by a second order approximation the term $u_{x} \cdot T\left(u_{z} \cdot T\right)$ must be known at the side-walls (upper and lower wall) of $\Omega(i, j)$. For a staggered grid the velocities are already present at these points. The corresponding temperatures, for example the temperature $T_{l}(i, j)$ at the left sidewall of the grid cell $\Omega(i, j)$ (e.g. at the position of $u_{x}(i, j)$ ), are calculated using

$$
\begin{array}{r}
T_{l}(i, j)=T(i, j)-F(a) \frac{T(i+1, j)-T(i, j)}{2} \\
\text { with } \quad a=\frac{T(i, j)-T(i-1, j)}{T(i+1, j)-T(i, j)}
\end{array}
$$

if $u_{x}(i, j)<0$ and

$$
\begin{array}{r}
T_{l}(i, j)=T(i-1, j)+F(a) \frac{T(i-1, j)-T(i-2, j)}{2} \\
\text { with } \quad a=\frac{T(i, j)-T(i-1, j)}{T(i-1, j)-T(i-2, j)}
\end{array}
$$

if $u_{x}(i, j)>0$, where

$$
F(a)=\max (0, \min (2 a, \min (1 / 3+2 / 3 a, 2))) .
$$

After the determination of the four temperature values $T_{l}, T_{r}$ (temperature at the right side of the cell), $T_{t}$ (temperature at the top of the cell) and $T_{b}$ (temperature at the bottom of the cell)

$$
\frac{\partial u_{x} \cdot T}{\partial x}=\frac{u_{x}(i+1, j) \cdot T_{r}(i, j)-u_{x}(i, j) \cdot T_{l}(i, j)}{h_{x}}
$$

can be calculated where $h_{x}$ is the grid size in the $x$-direction. The other derivative is obtained analogous.

Further explanations and some test results for this and other methods can be found in Koren (1993).

\subsubsection{Solution of the discretized equations}

The discretized Continuity and Stokes equations are solved using a Full Approximation Storage (FAS) Multigrid (MG) Algorithm with the Symmetric Coupled Gauss Seidel (SCGS) Method as smoother (see Auth and Harder, 1999).

For the Energy and Damage equations the Multigrid algorithm includes a defect correction scheme (Trompert and Hansen, 1996) and a simple Gauss-Seidel method as smoother.

The multigrid method is chosen, because

(1) it is comparatively robust for numerical problems with strongly varying viscosity (in contrast to spectral methods for example);

(2) the computation time scales linearly with the number of grid-points. 


\subsection{Test of the Algorithm}

Since no benchmark calculations using the proposed rheology are available, two kinds of test are performed to ensure the correctness of the computational code.

(1) The Energy and the Damage equations are processed by the same subroutines. The only differences are parameters for the diffusion, source and sink terms in both equations. For $d \equiv 0$ the program is successfully tested, using benchmarks from Blankenbach et al. (1989): Table 4.1 presents the comparison between my results and Christensen's for the internally

\begin{tabular}{|c|r|r|r|}
\hline Quantity & Results Christensen & Results Auth & Difference in \% \\
\hline \hline \multirow{3}{*}{$v_{r m s}$} & 60.3674 & 60.76 & 0.65 \\
& 31.9785 & 31.99 & 0.04 \\
& 57.4309 & 57.67 & 0.42 \\
& 30.3160 & 30.37 & 0.18 \\
\hline \multirow{3}{*}{$N u$} & 7.37845 & 7.444 & 0.89 \\
& 6.47106 & 6.502 & 0.48 \\
& 7.19712 & 7.261 & 0.89 \\
& 6.79493 & 6.835 & 0.59 \\
\hline Period & 0.048043 & 0.048802 & 1.58 \\
\hline
\end{tabular}

Table 4.1: Comparison between the results of my code $(33 \times 33$ grid) and Christensens code for case 3 in the benchmark paper from Blankenbach et al. (1989). $v_{r m s}$ : root mean square velocity, Nu: Nusselt number.

heated double-periodical case 3 . Since my code is able to reproduce the benchmark results and since differences in the treatment of the Energy and the Damage equation occur only in the source and the sink terms, it is reasonable, that any possible mistake in the damage equation could only be caused by these two terms.

(2) To ensure, that the source and sink terms in the damage equations are processed in the right way, I did a couple of very simple calculations. To test the source term, I removed the sink term from the damage equation, fixed the viscosity and the initial damage field to one, the $\dot{\varepsilon}$ field to $\dot{\varepsilon}=\sin (2 \pi x) \sin (\pi z)$ and performed just one time step. The new damage field can be compared with the analytical result. Similar tests with prescribed temperature fields are also done for the sink term.

\subsection{Numerical limitations}

One problem with this rheology is the drastic deterioration of convergence in the Multigrid algorithm for the Continuity and Stokes equations when strongly focused Low Viscosity Zones (LVZs) develop (as discussed for example in Auth and Harder, 1999). In my simulations self-focusing LVZs have typically a width of five grid-points and a viscosity 
contrast of up to five orders of magnitude with respect to the surrounding undamaged material. Therefore many pre- and post-smoothers (12-16) and a time step size significantly lower than the Courant Criterion (about a factor of 10 - 100) have to be used to ensure convergence. Nevertheless, the maximum absolute residuals of the Continuity and the Stokes equations are often reduced only by a factor of around 0.9 per MG-cycle.

Another numerical problem are negative values for the damage. It is clear from equation

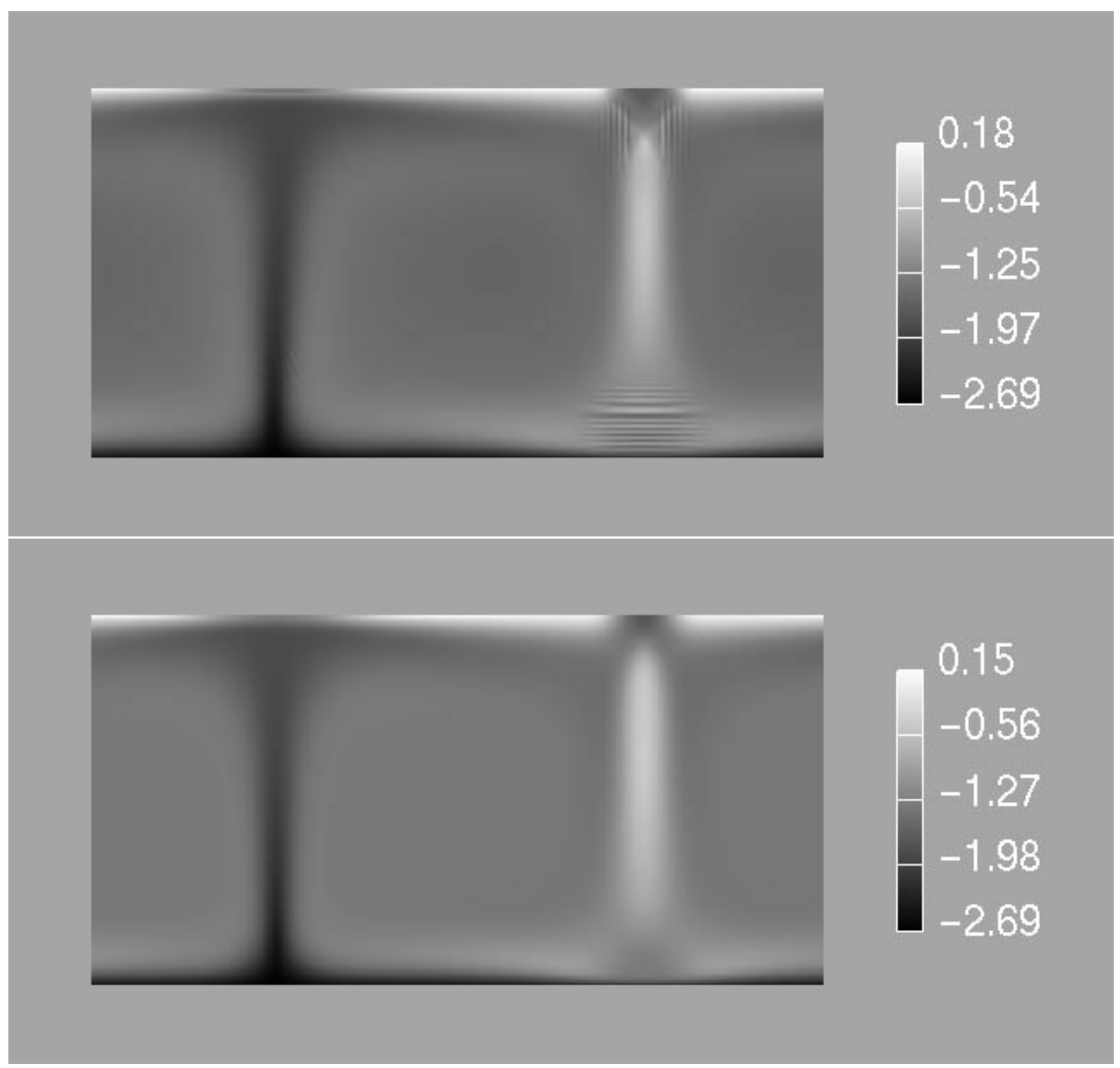

Figure 4.2: Top picture: Oscillations in a viscosity field if the time step size is too long. Bottom picture: The corresponding field without oscillations.

3.8 that the damage should always be positive, because the sink term vanishes for $d \rightarrow 0$ and becomes a source term for $d<0$. This is also intuitive, because negative grain sizes or negative porosities are not physical. However, "large" time-steps can produce negative damage at some grid-points. Their negative damage acts as an additional source term in 
the subsequent time-step and leads to a significant growth of the damage at these points. Thus, the sink term is large again in the third time-step and negative damage is obtained again, etc.. Figure 4.2 shows a typical example for the resulting oscillations in the viscosity field. These oscillations are suppressed by (1) reducing the time step size and (2) prohibiting negative damage values using the cut-off: $d(i, j)=c * \exp (d(i, j)-c)$ for all $d<c$ and with $c=10^{-12}$.

Both, the reduction of the time-step-size and the manipulation of the damage field are done automatically in the program. Typical orders of magnitude for the time step size reduction compared to the Courant criterion during periods of high damage are around ten to hundred.

\subsection{Resolution of the Low Viscosity Zones}

In this section I show that a low viscosity zone (LVZ) is sufficiently resolved, if each of its sides is sampled by at least three grid-points (including the point of minimum viscosity). Moreover, I demonstrate that even if this resolution is not obtained a "real existing" LVZ remains visible in the viscosity field.

\subsubsection{Grid-points per LVZ}

The aim of this test is to find out how many grid-points are required to sample a LVZ with an appropriate resolution.

I basically use the same method as presented earlier in my discussion damage advection (see 3.3.2). For a prescribed horizontal velocity field

$$
u_{x}=\frac{1000 c}{1+e^{c(x-z) / 0.01}}
$$

with $c=1$ and a vertical velocity field $u_{z}$ determined with the same formula, but using $c=-1$, the steady state damage field is calculated from the damage equation for different values of $a$ and $b\left(a=0.01 / a=0.1 / a=1, a / b=2 \cdot 10^{-5}\right.$ and $\left.m=1.1\right)$ and for different resolutions (see subsection 3.3.2, figure 3.3).

Figure 4.3 shows the steady state viscosity fields for $a=0.01$ and different resolutions $(17 \times 17,33 \times 33,65 \times 65$ and $129 \times 129$ grid points $)$ at a depth $z \approx 0.5^{2}$. The important difference between the four results are the values of the minimum viscosities and the following viscosity values downstreams. The solutions on the $129 \times 129$ and the $65 \times 65$ grids show nearly no differences. On the logarithmic scale the deviation between the minimum viscosities is less than 1 percent. This value increases to 3.8 percent deviation between the $129 \times 129$ and the $33 \times 33$ grids and to 10.9 percent between the $129 \times 129$ and the $17 \times 17$

\footnotetext{
${ }^{2}$ Because of the staggered grid the cuts through the viscosity fields are not exactly at the same depth; thus small horizontal translations of the different curves are negligible
} 


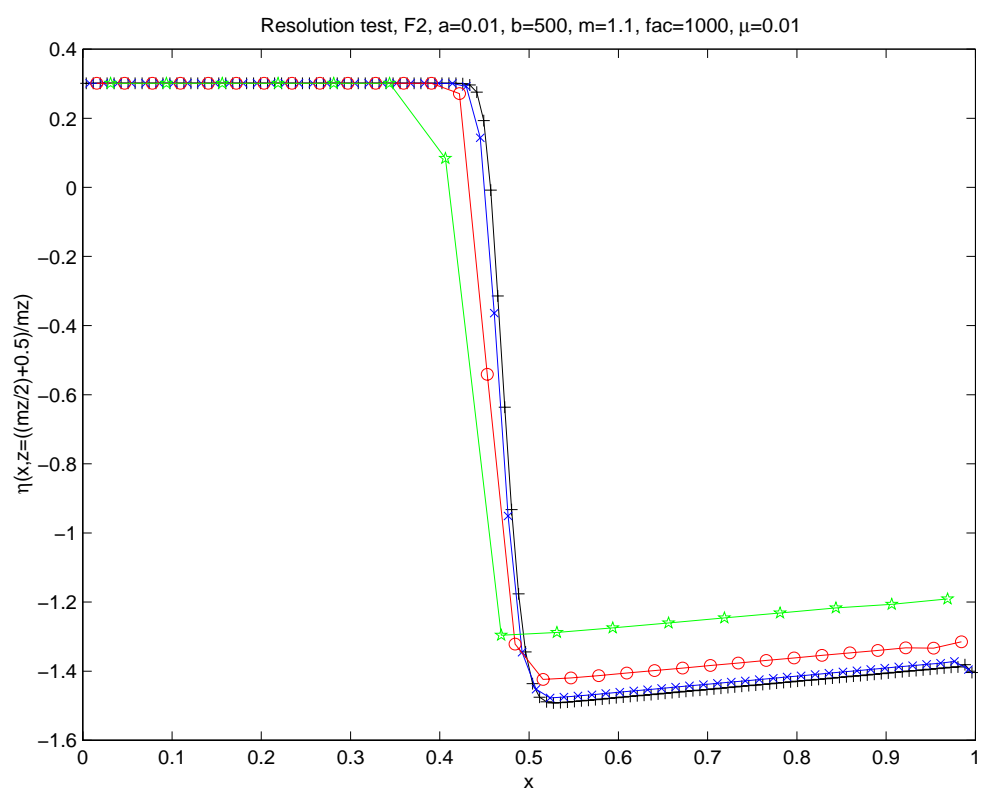

Figure 4.3: First resolution test. The steady state viscosity field at depth $z \approx 0.5$ obtained for a prescribed velocity field and $a=0.01, b=500, m=1.1$ on different grids $(17 \times 17$, $33 \times 33,65 \times 65$ and $129 \times 129)$.

grids. However, since deviations of less than five percent seem acceptable and considering the numerical effort for grid refinements, I assume that the $33 \times 33$ grid is appropriate to sufficiently resolve the LVZ.

The crucial point for the resolution problem is, how well the maximum of the strain rate is sampled. Here the upstream side of the LVZ is the side with the worse resolution (because the influence of advection is weaker than on the other side) and therefore the resolution of the maximum of the strain rate depends on the resolution of this side of the LVZ. Since it is sufficiently resolved by three grid-points in this case I assume that in general numerical problems are sufficiently resolved, if the worse resolved sides of all LVZs are sampled by at least three grid-points. As a consequence the LVZs in a properly resolved simulation are sampled by at least five grid-points.

This result is confirmed by resolution tests with different input parameters and is also consistent with results about the resolution needed for thermal boundary layers.

\subsubsection{Under-resolved LVZs}

Let us assume that in the true solution of a prescribed model problem some very narrow LVZs occur. Let us further assume that (because I did not know that) I started my calculation on a grid too coarse to appropriately sample these LVZs. What happens in this calculation? Is it possible that no influence of the damage parameter is obtained at all, because the zones where the damage could be high are narrower than the grid spacing? 
Figure 4.4 shows again the results of the previous resolution test, but this time on the grids

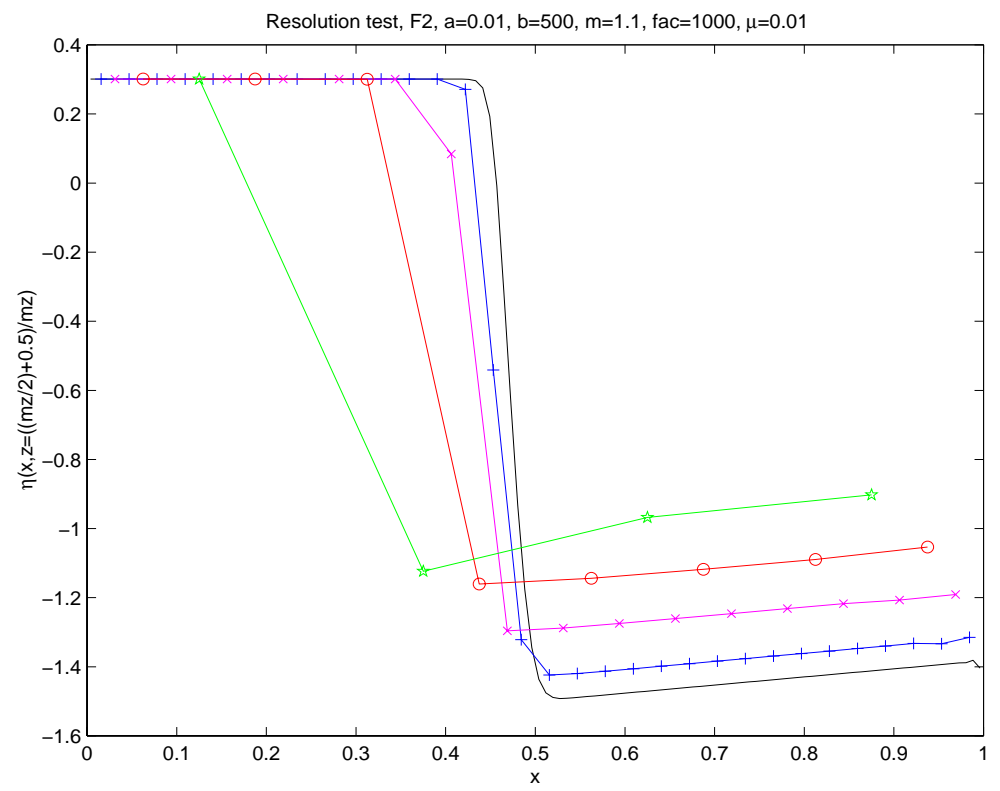

Figure 4.4: Second resolution test. Shown are the steady state viscosity fields at depth $z \approx 0.5$ obtained for a prescribed velocity field and $a=0.01, b=500, m=1.1$ on different grids $(5 \times 5,9 \times 9,17 \times 17,33 \times 33,129 \times 129)$.

$5 \times 5,9 \times 9,17 \times 17,33 \times 33$ and $129 \times 129$. It can be seen, that the LVZ becomes broader and its viscosity increases with the coarsening of the grid. However, even if the grid is much too coarse to sample the "true" zone of strong velocity changes (see grid $129 \times 129$ ) I still get a viscosity reduction. The reason is that the velocity fields here are prescribed independent of the underlying grid. For all the grids which are too coarse to sample the change in the velocity direction I still obtain non vanishing velocity differences between neighboring grid-points. Because the calculated strain rate on a grid also depends on the inverse cell size the source term in the damage equation decreases with the decreasing number of grid-points.

However in real simulations the velocity and viscosity fields are coupled. If the viscosity becomes higher in LVZs because of resolution problems the velocity does not change its direction abruptly any more. As a consequence the strain rate decreases and the viscosity further increases. It can therefore be expected that under-resolution of a problem acts against the self-lubricating effect. To check this expectation a resolution test using a real simulation is shown now.

This example (parameters: aspect ratio 2, $a=1, b=5 \cdot 10^{4}, m=2, R a=10^{4}, \gamma=\ln 10^{3}$, bottom heating) is calculated on $257 \times 129,513 \times 257$ and $1025 \times 513$ grids. Figure 4.5 shows viscosity fields for all three cases and horizontal cuts through the fields for the coarsest and the finest one. Since the case is time-dependent and the time dependence changes with resolution only compare general features can be compared: As expected, the LVZs are 

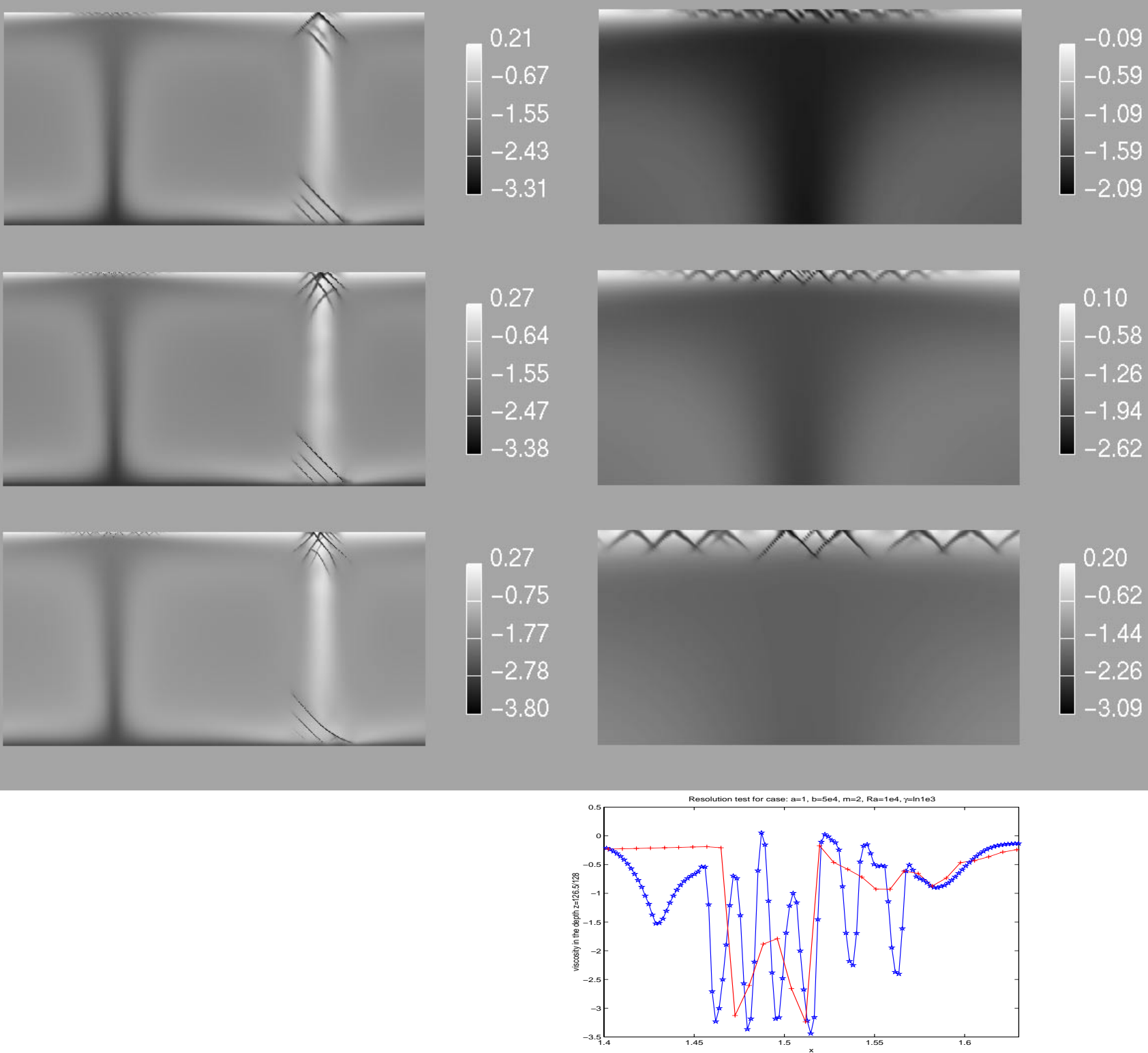

Figure 4.5: Resolution test for the following paprameters: $a=1, b=5 \cdot 10^{4}, \mathrm{~m}=2$, $R a=10^{4}, \gamma=\ln 10^{3}$. Left column: Viscosity fields for resolutions $257 x 129,513 x 257$ and $1025 x 513$ grid points. Right column: Zoom to the top of the upwelling. Last picture: Horizontal cut through the viscosity fields in the depth $z=126.5 / 128$ for resolutions $257 x 129$ and $1025 x 513$.

narrower in the better resolved cases and their viscosity is lower. Above the downwelling much more LVZs are present on the $1025 \times 513$ grid, than on the $257 \times 129$ grid. The horizontal cut through the viscosity field in the upwelling region shows that even on the $1025 \times 513$ grid the "v" LVZs in the middle of the upwelling are not properly resolved. These structures have therefore an extension significantly below the grid cell size of the 
$257 \times 129$ grid. Nevertheless LVZs above the upwelling are visible on that grid, although their "v" shape is hardly developed.

In summary a significant under-resolution in a simulation forces the LVZs to become broader and the viscosity inside to increase. Small-scale structures can fuse or become unrecognizable. However the influence of the damage remains always visible. The overlooking of major low viscosity structures because of resolution problems can therefore be excluded for the simulations presented in these thesis.

\subsection{Diagnostic Parameters}

In this section I introduce some quantities used to evaluate my simulations in terms of platelike behavior (homogeneous plate velocities, focused LVZs, asymmetrical subduction, ...).

(1) Most important to detect plate-like behavior are snapshots of the viscosity field during the calculation.

(2) The horizontal velocity field or at least the surface velocity structure is necessary to see the uniformity of the material movement at the surface.

However, for reasons of disc storage space and clarity of presentation, the number of snapshots I can show here is limited. Therefore some additional simple controlling parameters to reflect the temporal evolution of my model are necessary. In particular I use:

(3) the root-mean-square-velocity $\left(v_{r m s}\right)$ to detect (sudden) changes in the convective structure of the model,

(4) the maximum damage in the box $\left(d_{\max }\right)$ to see, whether significant influence of the damage is present at all,

(5) the number of "plate points", where a grid-point at the surface is defined to be a plate point, if the derivative of its horizontal velocity is at least a thousand times smaller than the maximum derivative of the horizontal surface velocity. The number of plate points is used to measure the uniformity of the surface velocity. Tackley's definition of plate points (Plateness $P=1-f_{80} / 0.6$, where $f_{80}$ is the fraction of surfcae area in which the highest $80 \%$ of the deformation occurs) (Tackley (2000c)) is not used here, since the factor of 0.6 is a Rayleigh number dependent guess for $f_{80}$ in isoviscous simulations. Since the Rayleigh number is variable here my definition seems to make more sense.

(6) the number of plates, where a plate is formed by at least two neighbouring plate points, (7) the maximum variation in the surface velocity $u_{\text {surf,max }}-u_{\text {surf,min }},(8)$ the horizontally averaged horizontal velocity at a particular depth, which can give informations about the asymmetry of convection. Since the horizontal velocity averaged over the whole domain is designed to vanish - given periodic boundary conditions - the line averaged horizontal velocity should always be zero for a perfectly symmetric convection pattern. I usually use the velocity averages at depth $z=0.25$ and $z=0.75$.

(9) the averaged temperature inside the computational domain. Especially for simulations 
with significant influence of damage this quantity is used to determine whether a (statistical) steady state is already reached. 


\section{Chapter 5}

\section{Bottom heated models}

In this chapter simulation using heating of the computational domain from below are presented.

\subsection{Basic Results}

The aim of this section is to understand the principal influence of the input parameters $a$ (source of damage), $b$ (sink of damage) and $m$ (exponent of the damage in the viscosity law) onto the convective structure of a bottom heated system.

\subsection{Advanced Results}

In this second section I optimize the plate-like behavior based on my previous results.

\subsubsection{Summary of the results for bottom heated models}

This section finally summarizes and organizes the results obtained from the study of bottom heated systems. 


\subsection{Basic Results}

\subsubsection{Introduction}

The goal of the following part of this paper is to present the changes in the convection pattern and the plate-like behavior in my simulations due to variations of single input parameters. This is done in four series, varying $a, a$ and $b$ simultaneously and finally varying $m$ (two series). $R a=10^{4}, \gamma=\ln 1000$ and the box aspect ratio of two are kept constant throughout the chapter ${ }^{1}$.

\begin{tabular}{c|r|rrr|c} 
Series & Case-\# & $a$ & $b$ & $m$ & Regime \\
\hline \multirow{4}{*}{ A0 } & 1 & $3.3 \cdot 10^{-2}$ & $5 \cdot 10^{4}$ & 1.1 & I \\
& 2 & 0.10 & $5 \cdot 10^{4}$ & 1.1 & II \\
& 3 & 1.00 & $5 \cdot 10^{4}$ & 1.1 & IIIa \\
& 4 & 5.00 & $5 \cdot 10^{4}$ & 1.1 & IIIa \\
\hline \multirow{6}{*}{ AB } & 5 & 10.0 & $5 \cdot 10^{4}$ & 1.1 & IV \\
& 1 & 0.01 & $5 \cdot 10^{2}$ & 1.1 & IIIa \\
& 2 & 0.10 & $5 \cdot 10^{3}$ & 1.1 & IIIa \\
& 3 & 1.00 & $5 \cdot 10^{4}$ & 1.1 & IIIa \\
& 4 & 10.0 & $5 \cdot 10^{5}$ & 1.1 & IIIa \\
& 5 & 20.0 & $1 \cdot 10^{6}$ & 1.1 & IV \\
\hline \multirow{4}{*}{ M0 } & 1 & 1.00 & $5 \cdot 10^{4}$ & 0.7 & IIIa \\
& 2 & 1.00 & $5 \cdot 10^{4}$ & 1.1 & IIIa \\
& 3 & 1.00 & $5 \cdot 10^{4}$ & 1.3 & IIIa \\
& 4 & 1.00 & $5 \cdot 10^{4}$ & 1.4 & IIIb \\
& 5 & 1.00 & $5 \cdot 10^{4}$ & 1.5 & IIIb \\
& 6 & 1.00 & $5 \cdot 10^{4}$ & 2.0 & IIIb \\
\hline \multirow{6}{*}{ M1 } & 1 & 0.04 & $2 \cdot 10^{3}$ & 1.1 & IIIa \\
& 2 & 0.04 & $2 \cdot 10^{3}$ & 2.0 & IIIa \\
& 3 & 0.04 & $2 \cdot 10^{3}$ & 3.0 & IIIb \\
& 4 & 0.04 & $2 \cdot 10^{3}$ & 4.0 & IIIb
\end{tabular}

Table 5.1: Overview over the parameter studies in the section 5.1.

An overview of these presented calculations is given in table (5.1). Note, that the same case can have different names if it is included into different series. For example the names A0-3, AB-3 and M0-2 all refer to the same case.

The simulations are long enough to become independent from their initial conditions.

All LVZs in the calculations are properly resolved, if not stated otherwise. A cut off of the

\footnotetext{
${ }^{1}$ As introduced in section 3.1 the Rayleigh number here is scaled with the surface viscosity $\eta_{0}(T=0, d=1)$; a viscosity scale given by $\eta$ at a typical mantle temperature of $1500^{\circ}$ Celsius (or 0.5 in non-dimensional quantities), as done by several other authors, would result in an effective Rayleigh number of around $3 * 10^{5}$.
} 
viscosity field ${ }^{2}$ is never performed.

\subsubsection{Variation of $a$, series A0}

In the first series of numerical experiments $(\mathrm{A} 0)$, the influence of the damage source term parameter $a$ shall be tested. I fix the healing term parameter to $b=5 \cdot 10^{4}$ and the exponent of the damage parameter in the viscosity law equation (3.7) to $m=1.1$, which is greater than unity to allow self-lubricating behavior (see my analysis in the section "Features of the used rheology"). The parameter $b$ is chosen such, that the damage parameter $d$ in material moving with a non-dimensional velocity of 1000 (corresponding to around $1 \mathrm{~cm} /$ year) would decay to $d / e$ within a distance equal to $1 / 50^{t h}$ of the length of the box (assuming no source terms for $d$ ). For the size of the parameter $a \mathrm{I}$ follow the results of Tackley (1998), who used a rheology similar to the asymptotic one in equation (3.11), and found an $a / b$ ratio of $10^{-5}$ as being adequate for producing LVZs. I present here results for $a=0.1, a=1.0$ and $a=10.0$.

For $a \approx 0.033$ the source term in equation (3.8) is too small to allow $d$ to become significant and the system tends to steady state with only temperature-dependent viscosity and no plate-like behavior.

For $a=0.1 \mathrm{I}$ observe episodic behavior. During long periods the convection shows very weak upwellings or downwellings, cold material is accumulated in the upper thermal boundary layer and the damage is negligible. When the unstable density stratification collapses, fast upwellings and downwellings are produced, accompanied by a strong increase in the damage parameter (Figures 5.1 and 5.2). A tendency toward plate-like behavior is obtained only during the periods of fast subduction. I find little asymmetry in the upwellings and downwellings, but the surface velocity of the material clearly differs from a sinusoid, i.e. it hardly varies in regions between the upwelling and downwelling, but it changes very rapidly above the downwelling The change above the upwelling is less abrupt.

The case $a=1.0$ produces a steady state with constant influence of the damage parameter; figure 5.1 clearly shows the viscosity reduction above the downwelling for this case. A sharp change in the surface velocity above the downwelling is obtained and a little broader change above the upwelling. Between these zones the velocity hardly varies. However, essentially no asymmetry can be found in upwelling or downwelling.

Although I get time-dependent behavior for $a=10.0$, general features of the convection pattern are stable: The influence of the damage parameter remains significant in large parts of the box. Above the upwelling a broad reduction of viscosity can be seen and the LVZ in the downwelling region is more extensive than for $a=1.0$ and covers the downwelling like a lens. Figure 5.1 shows, that in this case both the upwelling and downwelling have asymmetric structure. The convection in the cell in the middle of the box is much more vigorous than in the adjacent cell (see surface velocity) and the averaged horizontal velocities at

\footnotetext{
${ }^{2}$ That means the viscosity is not allowed to decrease below a prescibed value
} 

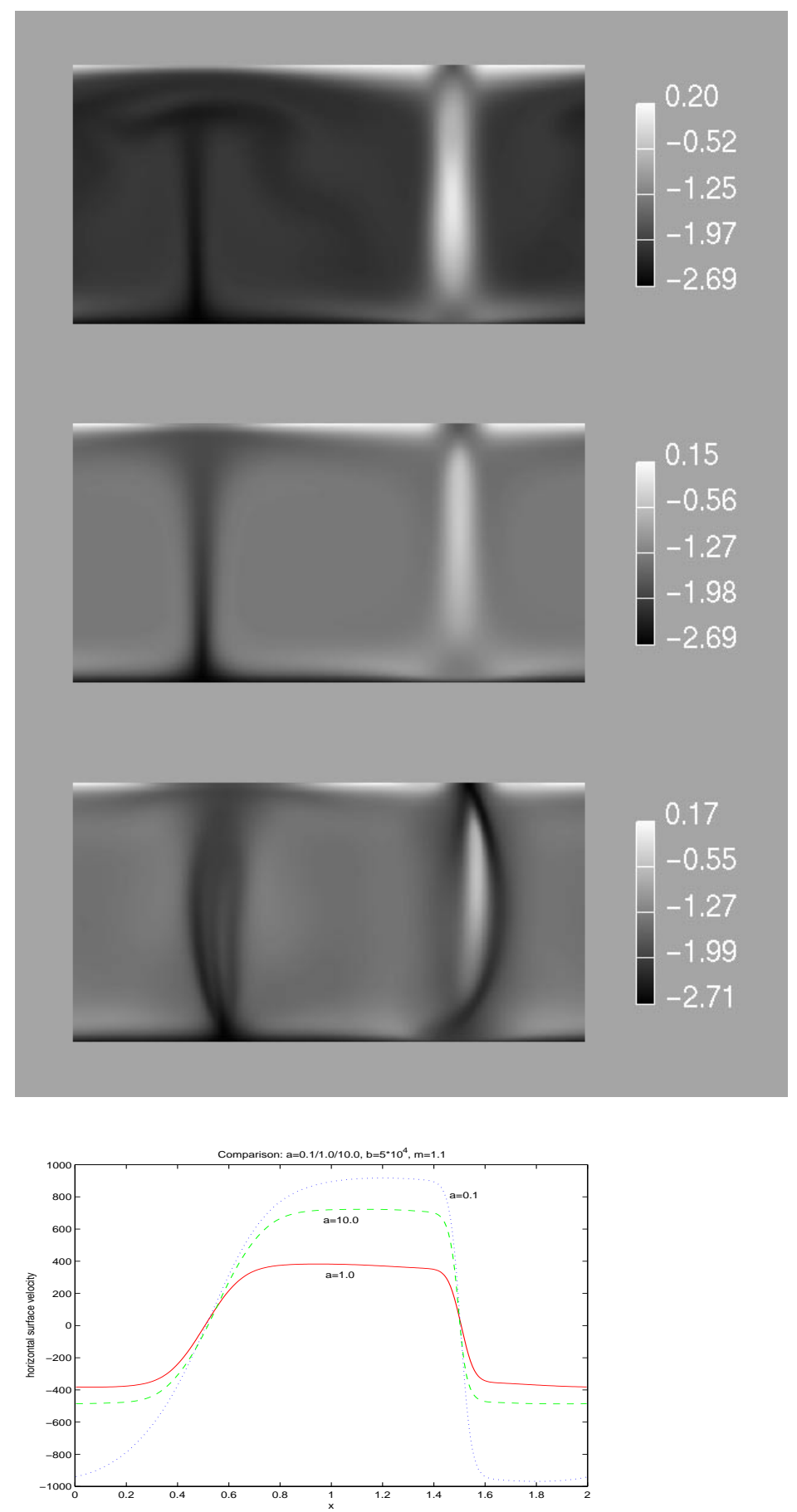

Figure 5.1: Series A0. From top to bottom: Viscosity snapshots for cases A0-2 ( $a=0.1$, during a subducting episod), A0-3 ( $a=1.0$, steady state field) and A0-5 ( $a=10.0$ at a rms-velocity maximum). Constant parameters: $b=5 \cdot 10^{4}, m=1.1$. Last picture: Surface velocity for the three cases. 


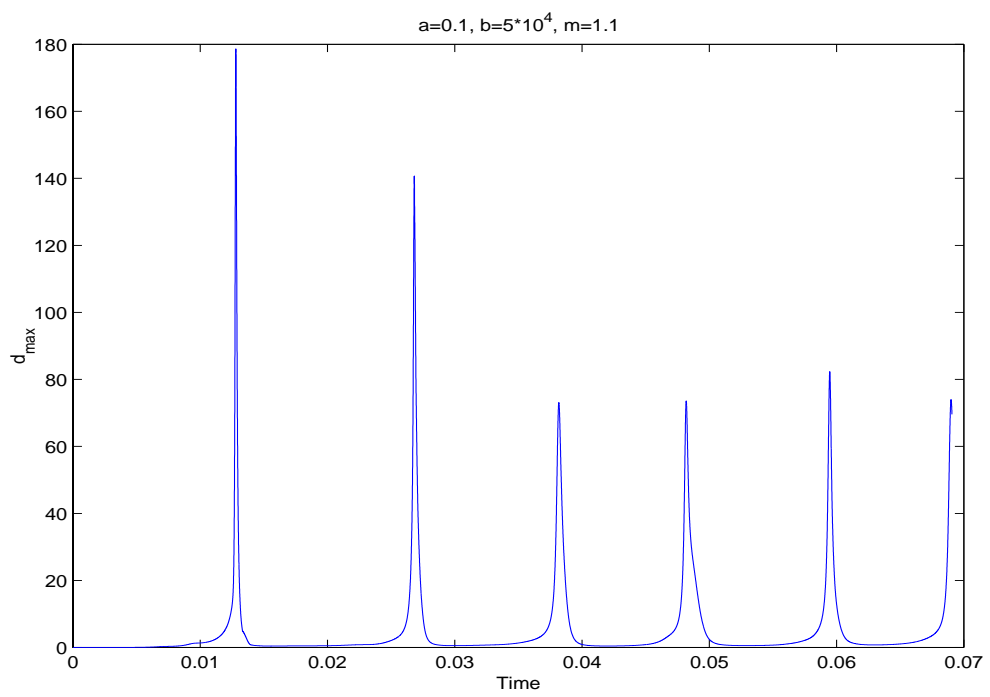

Figure 5.2: Maximum damage over Time for case A0-2 $\left(a=0.1, b=5 \cdot 10^{4}, m=1.1\right)$

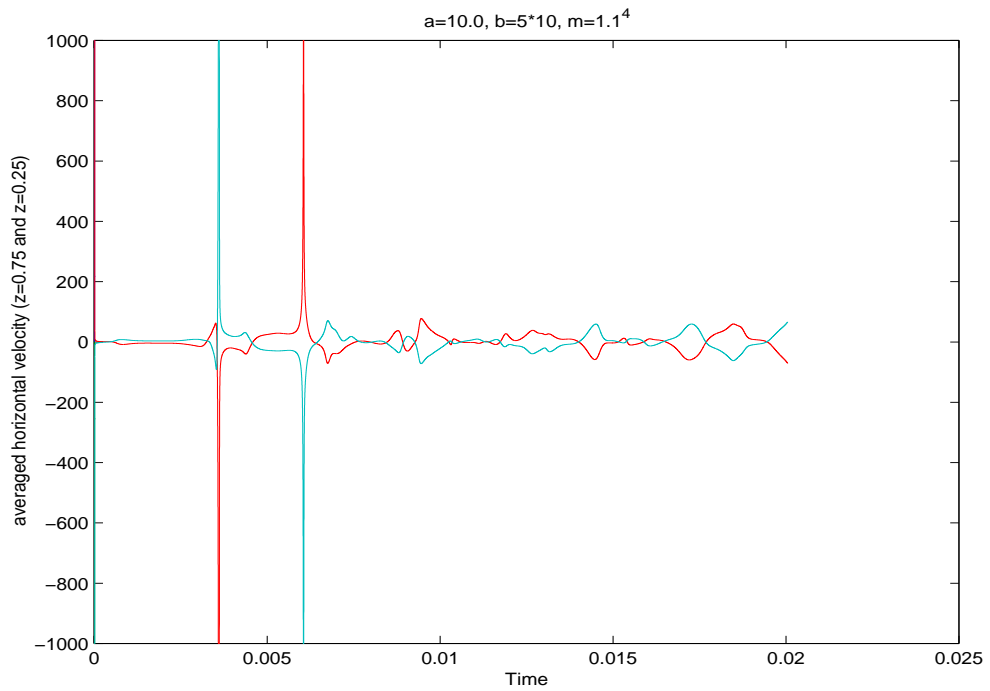

Figure 5.3: Velocity in $x$-direction horizontally averaged for depths $z=0.25$ and $z=0.75$ versus Time for case $A 0-5\left(a=10.0, b=5 \cdot 10^{4}, m=1.1\right)$

depth $z=0.25$ and $z=0.75$ plotted in figure 5.3 demonstrate, that this behavior oscillates in time. Since the lithosphere is weakened in a broad zone above the upwelling the surface velocity change above the upwelling becomes broader than, for example, in the case $a=1.0$. Nevertheless the surface velocity change above the downwelling remains very sharp and the velocity variations between upwelling and downwelling are less gradual than for smaller $a$. 


\subsubsection{Simultaneous variation of $a$ and $b$, series AB}

In a second series of experiments $(\mathrm{AB})$ we focus on the influence of the advection term. Thus I choose $m=1.1, a / b=2 \cdot 10^{-5}$ and $a$ and $b$ are varied simultaneously using $a=0.01 ; 0.1 ; 1 ; 10 ; 20$.

I observe, that the steady state viscosity fields for the different $a$ and $b$ values differ significantly from each other only above the downwelling (figure 5.4).

For $a=0.01 \mathrm{I}$ see an extended LVZ with a maximum value of the damage parameter $d$ of around 30 and a maximum strain rate of $\dot{\varepsilon}_{\text {calc }} \approx 14000^{3}$.

In the next three cases the extent of the low viscosity zone is reduced relative to the $a=0.01$ case and the maximum value for $d$ is increased to approximately $70\left(\dot{\varepsilon}_{\text {calc }} \approx 13500\right)$. The shape of the LVZ changes from a linear structure for $a=0.1$ to a more "v"-like structure for higher $a$.

The case $a=20$ however is different. It is not only weakly time-dependent, but also shows focused LVZs with lower viscosity than obtained in the other cases. Instead of one extended "v"-shaped LVZ, there are a few smaller weak zones that are parallel to or crossing one another. The maximum value for the damage parameter in the subduction zone increases to around 240 and $\dot{\varepsilon}_{\text {calc }} \approx 63000$.

Despite the differences in the LVZs the general convective pattern, the root-mean-square and the surface velocity are nearly identical in all cases, except that the surface velocity variation above the downwelling is more inhomogeneous in the last case (AB-5) and occurs in small jumps, forming "micro-plates" in between (figure 5.4). No significant asymmetry in the downwelling is visible in any of the cases.

However, two points can be learned from this series: (1) A good quantity to determine the boundary between non-self-lubricating and self-lubricating behavior is the maximum strain rate $\left(\dot{\varepsilon}_{\text {calc }}\right)$. It appears to be nearly constant in the non-self-lubricating regime but increases significantly when self-lubricating occurs. (2) Because of the influence of the advection term in the damage equation the strain rate which has to be exceeded before self-lubricating occurs $\left(\dot{\varepsilon}_{s l}(a, b, m)\right)$ is much higher, than the corresponding strain rate $\left.\dot{\varepsilon}_{\max }(a / b, m)\right)$ obtained from my analysis without advection. In this series we do not get self-lubricating for $\dot{\varepsilon}_{\text {calc }} \approx 14000$ although $\dot{\varepsilon}_{\text {max }} \approx 2000$ can be assumed from figure 3.2 .

\footnotetext{
${ }^{3}$ The suffix calc is just meant to distinguish this calculated maximum strain rate $\dot{\varepsilon}_{\text {calc }}$ from the analytically obtained strain rate $\dot{\varepsilon}_{\text {max }}$ in section 3.3
} 

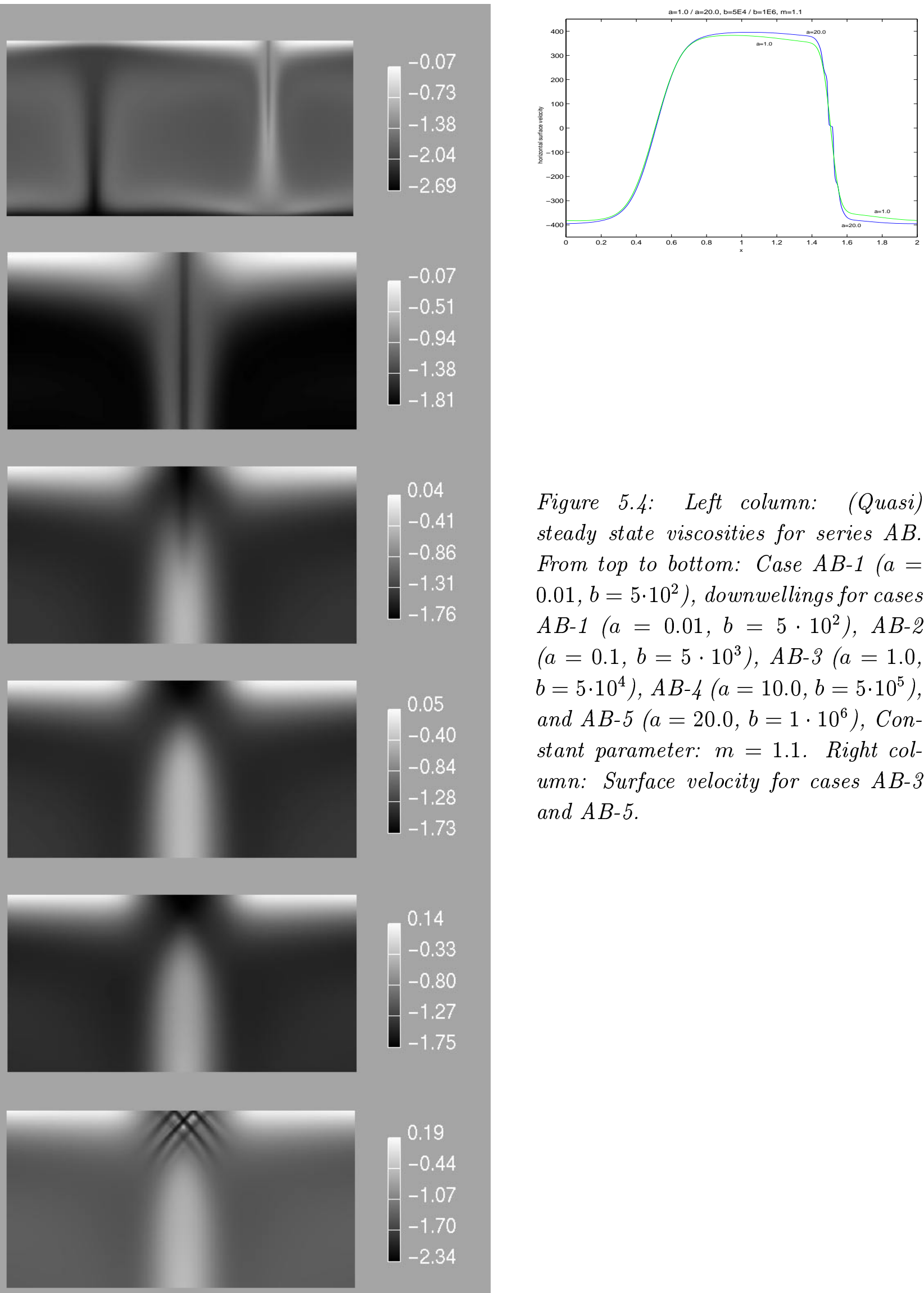


\section{Variation of $m$, Series Mo}

The last parameter I investigate is the exponent of the damage parameter in the viscosity law, $m$. For $a=1.0$ and $b=5 \cdot 10^{4}$ I present the cases $m=0.7 ; 1.1 ; 1.3 ; 1.4 ; 1.5 ; 2.0$ in figure 5.5.

For $m=0.7$ and $m=1.1 \mathrm{I}$ obtain a steady state solution with broad LVZs above and below the downwelling and $\dot{\varepsilon}_{\text {calc }} \approx 13500$. Case M0-3 $(m=1.3)$ has weak periodic time-dependence but similar LVZs and $\dot{\varepsilon}_{\text {calc }} \approx 14200$. For case M0-4 $(m=1.4)$ I see first indications for selflubricating behavior: The broad LVZs at the downwelling begin to focus to a few connected narrow LVZs with significant lower viscosity than for case M0-3. A similar process starts above the upwelling, although this is hardly visible in figure 5.5. The maximum strain rate reaches values up to $\dot{\varepsilon}_{\text {calc }} \approx 24500$. For a further increase in $m$ the LVZs continue to focus, their viscosity further decreases and $\dot{\varepsilon}_{\text {calc }} \approx 55000$ for $m=1.5$ and $\dot{\varepsilon}_{\text {calc }} \approx 315000$ for $m=2.0$ are obtained.

From the magnified parts of case M0-6 $(m=2.0)$ two interesting features can be seen. (1) All LVZs for $m<2.0$ are oriented in an angle of $45^{0}$ to the surface. However for $m=2.0$ a few LVZs in the downwelling also show angles around $60^{\circ}$. (2) The LVZs at the bottom of the downwelling are asymmetric for all calculation with $m>1.3$. This asymmetry is a stable feature, although the orientation of the asymmetry can change in time. However, I do not want to over-interpret the case M0-6, because parts of the narrowest LVZs are resolved by fewer than five grid points.

The focusing of the LVZs above the upwelling and downwelling leads to more discontinuous variations in the surface velocity and the development of micro-plates. Although the cases with $m>1.3$ are time-dependent the periods of asymmetry are short and this asymmetry is not well developed.

The result, that the transition between simulations without and with self-focusing LVZs occurs at $m \approx 1.4$ is somewhat unexpected. From my previous analysis (section 3.3 ) one might think, that this transition occurs at $m=1.0$, since this is the value, where this analysis predicts a change in the material behavior and since $\dot{\varepsilon}_{\text {calc }} \approx 13500$ is high enough to allow this change (see figure 3.2). But I have seen in the previous section, that the absolute values of $a$ and $b$ are not high enough to produce self-focusing behavior at $m=1.1$. So why does the transition to self focusing behavior happen at higher $m$ ? Although the advection term in my simple analysis is neglected (section 3.3), the general dependence of $\dot{\varepsilon}_{\text {max }}(m)$ (equation 3.12)seems to be correct: $\dot{\varepsilon}_{s l}$ decreases with increasing $m$. So self-focusing can not occur at $m=1.1$ because the advection of damage is too strong, but it can occur at $m=1.4$. In short, self-focusing occurs at higher than expected $m$ to adjust for the influence of advection. 

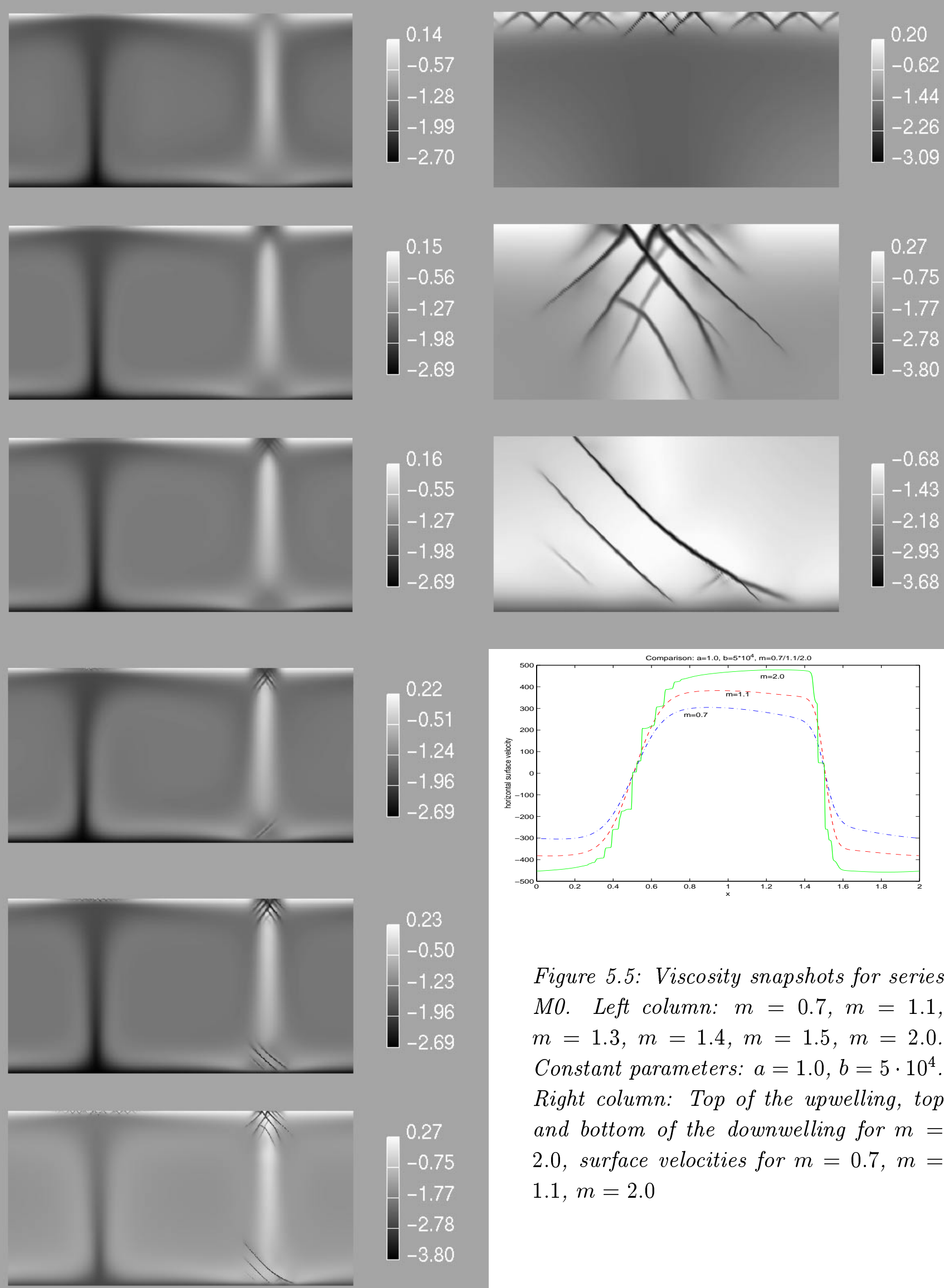

Figure 5.5: Viscosity snapshots for series MO. Left column: $m=0.7, m=1.1$, $m=1.3, m=1.4, m=1.5, m=2.0$. Constant parameters: $a=1.0, b=5 \cdot 10^{4}$. Right column: Top of the upwelling, top and bottom of the downwelling for $m=$ 2.0 , surface velocities for $m=0.7, m=$ 1.1, $m=2.0$ 


\section{Variation of $m$, Series M1}

In series M0 the values of $a$ and $b$ are quite high. Thus the influence of advection in the damage equation is low and the structures of the LVZs above the upwelling and downwelling are more "v"-like than linear, even for small $m$. Of course the increase of $m$ produces selffocusing of the LVZs, but their general "v" shape is not changed.

In my second series M1 I will show that for lower values of $a$ and $b$ an increase of $m$ is still able to change the structure of the LVZs from more linear to more "v"-shaped. I now choose $a=0.04$ and $b=2000$. The results for $m=1.1 ; 2.0 ; 3.0 ; 4.0$ show significant changes in the viscosity field (figure 5.6).

For $m=1.1$ and $m=2.0$ the LVZs above the downwelling are linear and I have a horizontally extended LVZ above the upwelling; however, there are no LVZs on the bottom of the box.

The cases with $m=3.0$ and $m=4.0$ in contrast show "v"-shaped LVZs above the downwelling, an additional LVZ beneath it and indications of a focused LVZ above the upwelling. Even so the influence of the small $a$ and $b$ values remain visible. The "v"-shaped LVZs above the downwelling are arranged one beneath the other, while they are side by side and connected for large $a$ and $b$ values. To explain this change in the convection pattern with increased $m$ we note that $m$ is not only included in the viscosity law, but also in the source term of damage equation (3.8) which appears as $a \frac{2}{1+d^{m}} e^{-\gamma T} \dot{\varepsilon}^{2}$. If a calculation is initiated with a linear damage structure (like that for $m=1.1$, for example) the positions for the maximum $d$ and the maximum $\dot{\varepsilon}$ values are not identical, since $d$ is maximum close to the middle of the subduction zone and $\dot{\varepsilon}$ is maximum where the velocity changes most abruptly. Thus the damage parameter $d$ will increase much more in the regions of high $\dot{\varepsilon}$ than in the regions of already high $d$. This effect obviously becomes stronger, as $m$ increases. So an increase in $m$ acts against advection and tends to pin the high damage zones to the high strain-rate zones. For each $m$ value, a balance is reached between pure linear structures (influenced primarily by advection) and pure "v" structures (influenced by strain-rate). Even in the more linear structure for $m=2.0$ a "v" pattern is visible, and even for $m=4.0$ the "v" structures are arranged one beneath another.

As with my results in varying $a$ and $b$ simultaneously, the changes in the "plate-like" behavior of the system are not significant. The surface velocity is similar for all cases and no micro-plates occur. Although $m=3.0$ and $m=4.0$ produce time-dependent behavior the asymmetry in the subduction zone is negligible.

\subsubsection{Summary of the parameter variations}

The increase of $a$ with fixed $b$ and $m$ improves the asymmetry of the system, but values that are too high can weaken large parts of the lithosphere thereby reducing the areas of plate-like homogeneous surface velocities. The absolute values of $a$ and $b$, for $a / b$ and $m$ constant, influence the shape of the LVZs above the upwelling or downwelling material. "Lower" values of a lead to linear structures, "higher" values to "v" structures. Temporary 

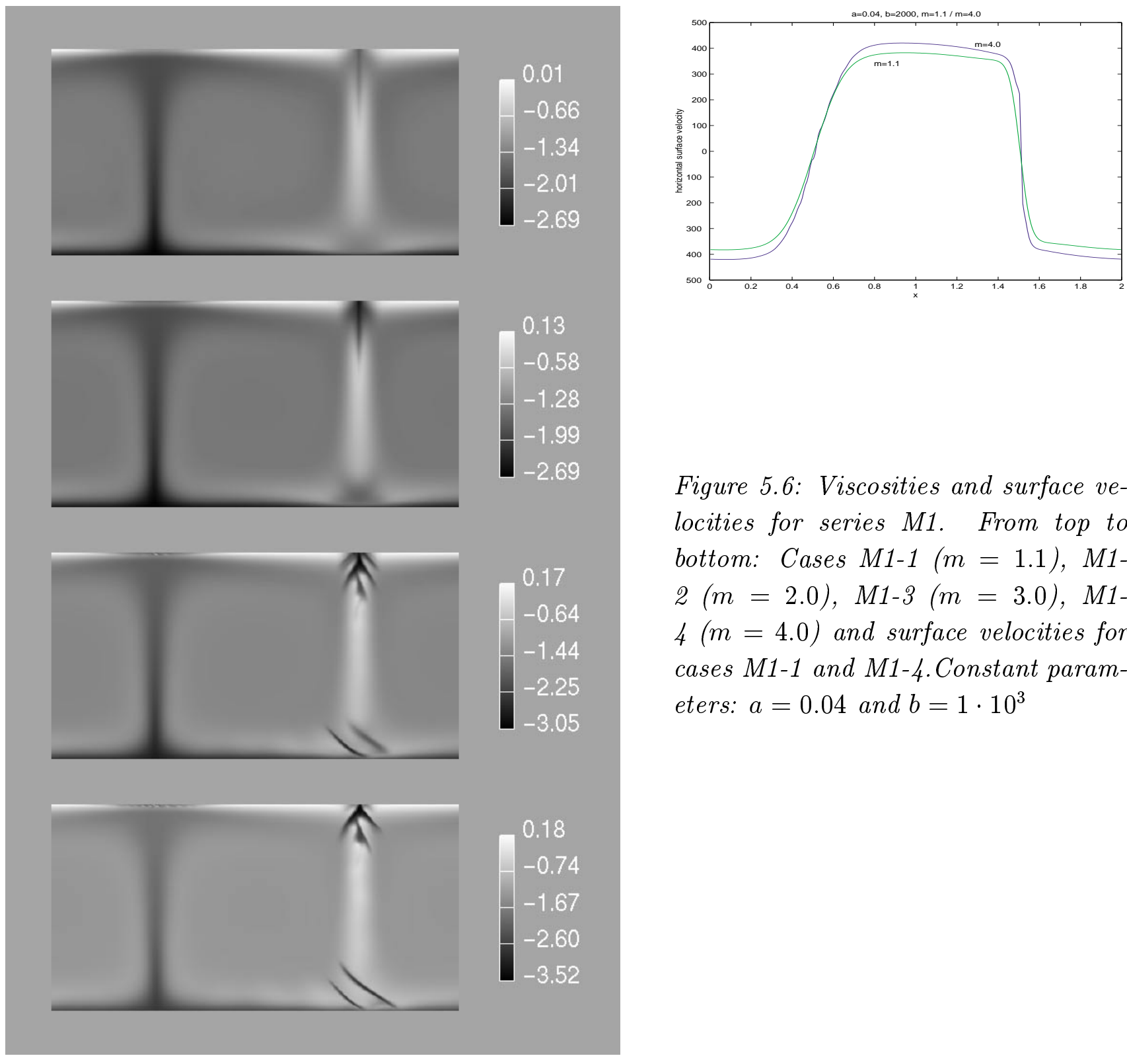

Figure 5.6: Viscosities and surface velocities for series M1. From top to bottom: Cases M1-1 ( $m=1.1)$, M1$2(m=2.0), M 1-3(m=3.0), M 1$ $4(m=4.0)$ and surface velocities for cases M1-1 and M1-4. Constant parameters: $a=0.04$ and $b=1 \cdot 10^{3}$

asymmetry and very focused LVZs are obtained, when the system enters the self-lubrication regime. What is meant by "lower" and "higher" values of $a$ and $b$ depends on the parameter $m ; m$ therefore can be used to control whether a calculation is in the self-lubricating regime and to get a suitable focusing of the LVZs. 


\subsection{Advanced Results}

\subsubsection{Introduction}

In the last section I discussed how the input parameters of my model influence the structure of convective flow. In this section I will use these results to optimize the plate-like behavior of my simulations by choosing appropriate parameter values. Unfortunately a lot of potentially interesting cases cannot be calculated because of numerical difficulties. For high values of $a$, $b$ and $m$ the LVZ are too small and their viscosity is too low to ensure their proper resolution and a sufficient convergence of my numerical algorithm. Thus I have to find a middle course between the optimum plate-like behavior and my numerical capabilities.

My new series are mainly motivated by the following two results:

(1) For $m>1.0$ high values of $a$ and $b$ can produce very focused LVZs but this drastically decreases the convergence of my numerical methods.

(2) Asymmetry of subduction improves when $a$ is increased.

Therefore my plan is to consider cases which have a good potential for self-lubrication (sufficiently high $m$ ), but are easy to compute ( $a$ and $b$ not too high), and then to slowly increase the asymmetry in the convective structure (increase $a$ ).

My first series A1 will start with case A1-1: $a=0.04, b=2000$ and $m=2.0$ (identical

\begin{tabular}{c|r|rrr|c} 
Series & Case-\# & $a$ & $b$ & $m$ & Regime \\
\hline \multirow{4}{*}{ A1 } & 1 & 0.04 & $2 \cdot 10^{3}$ & 2.0 & IIIa \\
& 2 & 0.08 & $2 \cdot 10^{3}$ & 2.0 & IIIa \\
& 3 & 0.10 & $2 \cdot 10^{3}$ & 2.0 & IIIa - IIIb \\
& 4 & 0.12 & $2 \cdot 10^{3}$ & 2.0 & IV \\
& 5 & 0.16 & $2 \cdot 10^{3}$ & 2.0 & IV \\
& 6 & 0.20 & $2 \cdot 10^{3}$ & 2.0 & IV \\
\hline \multirow{4}{*}{ A2 } & 1 & $8 \cdot 10^{-3}$ & $1 \cdot 10^{4}$ & 2.0 & I \\
& 2 & $16 \cdot 10^{-3}$ & $1 \cdot 10^{4}$ & 2.0 & II \\
& 3 & 0.04 & $1 \cdot 10^{4}$ & 2.0 & IIIb \\
& 4 & 0.20 & $1 \cdot 10^{4}$ & 2.0 & IIIb \\
& 5 & 0.40 & $1 \cdot 10^{4}$ & 2.0 & IIIb \\
& 6 & 0.50 & $1 \cdot 10^{4}$ & 2.0 & IV \\
\hline \multirow{4}{*}{ A3 } & 1 & 1.00 & $5 \cdot 10^{4}$ & 1.5 & IIIb \\
& 2 & 4.00 & $5 \cdot 10^{4}$ & 1.5 & IIIb \\
& 3 & 5.00 & $5 \cdot 10^{4}$ & 1.5 & IV
\end{tabular}

Table 5.2: Overview over the parameter studies in the section "Advanced Results"

to case M0-2). Since one of my problems with this series will be the focusing of the LVZs above the upwelling the next series A2 will use higher values for $a$ and $b$. A further increase of $a$ and $b$ is numerically possible only, if $m$ is lowered to $m=1.5$ (see discussion of series M0). The start of series A3 is therefore A3-1 (equal to M0-5): $a=1.0, b=5 \cdot 10^{4}, m=1,5$. 


\subsubsection{Series A1}

Starting with A1-1 $(a=0.04)$ I present cases with $a=0.04 ; 0.08 ; 0.10 ; 0.12 ; 0.16 ; 0.20$.

The cases A1-1 $(a=0.04), \mathrm{A} 1-2(a=0.08)$ and A1-3 $(a=0.10)$ produce steady state convection (figure 5.7).

For Case A1-4 $(a=0.12)$ the convective structure of the system changes significantly and

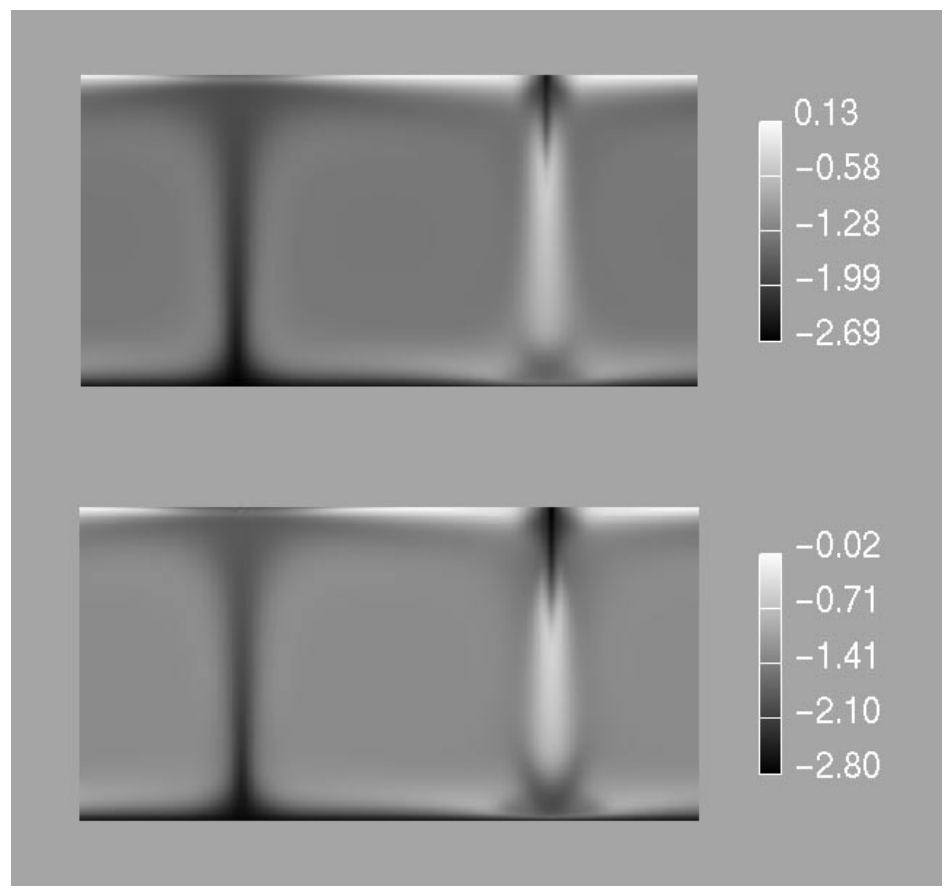

Figure 5.7: Steady state viscosities for cases A1-1 (a=0.04), and A1-3 $(a=0.10)$.

episodic behavior is obtained as shown in figure 5.8. In addition figure 5.10 presents snapshots from the evolution of the viscosity field between two (temporal) maxima of $d_{\text {max }}$. Figures 5.8 and 5.10 elucidate the reason for the episodic behavior: When only a little material is still subducted, a LVZ is present above the subduction zone in the region of high strain rate (second picture in figure 5.10). After the first cold subducting material reaches the bottom, LVZs develop there, too, and if the parameter $a$ is high enough, the shear stresses on the sides of the subducting material can be high enough to connect the top and bottom LVZ (third, forth and first picture in figure 5.10. The coupling between the subducting material and the surrounding material decreases, the subduction becomes faster, causing higher shear stresses on the sides of the subduction zone, the viscosity further decreases, etc. Finally the subducting material is so fast and the coupling to the material on the top of the box is so weak, that the slab breaks (second picture in figure 5.10). The rest of the old slab sinks to the bottom of the box, a new subduction starts again on the same position immediately.

How "plate-like" is the behavior of the material in this model? Although the LVZ above the downwelling has a more linear structure (comparatively low $a$ and $b$ values) the subduction zone is clearly asymmetric (figure 5.10). The plate-like quality of the surface velocity however 


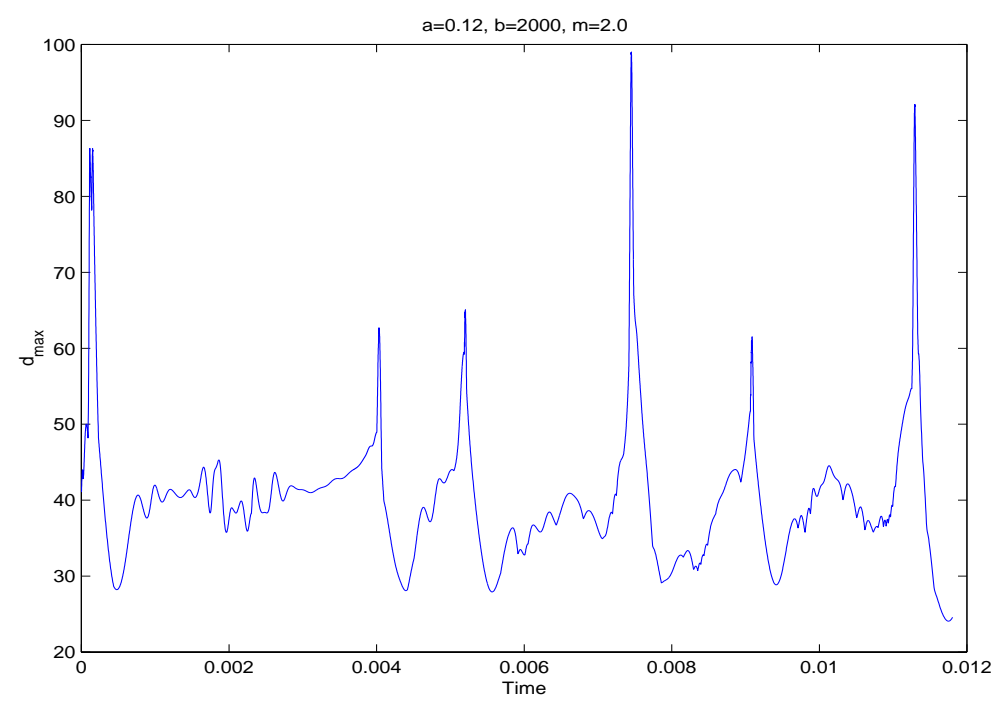

Figure 5.8: Maximum damage over Time for case A1-4 $(a=0.12, b=2000, m=2.0)$

is quite variable. Immediately after the fast subduction of large amounts of cold material into the mantle, the surface material in a large region around the upwelling is hot and has a high damage parameter, so it is quite weak and does not move with homogeneous velocity (figure 5.10). Because of the lower velocity (less slab pull) in the box the region of hot and highly damaged material on the surface diminishes in the following time and the material becomes more rigid in larger parts of the box. When the velocity and the damage parameter start to increase again, those parts subduct with homogeneous velocity and the weak zones above the upwelling grow up again. These variations in the homogeneity of surface velocity can be seen from plotting the relative number of plate-points versus time in figure 5.9: We get nearly $15 \%$ of the surface grid points to be plate-points during periods of

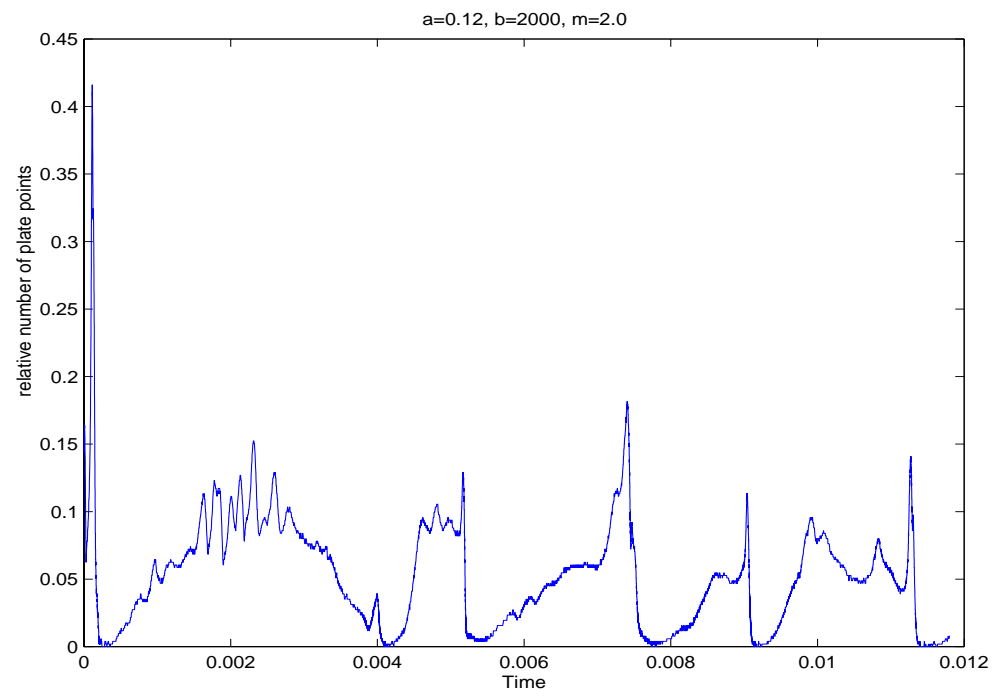

Figure 5.9: Relative number of Plate points for case A1-4 $(a=0.12, b=2000, m=2.0)$ 

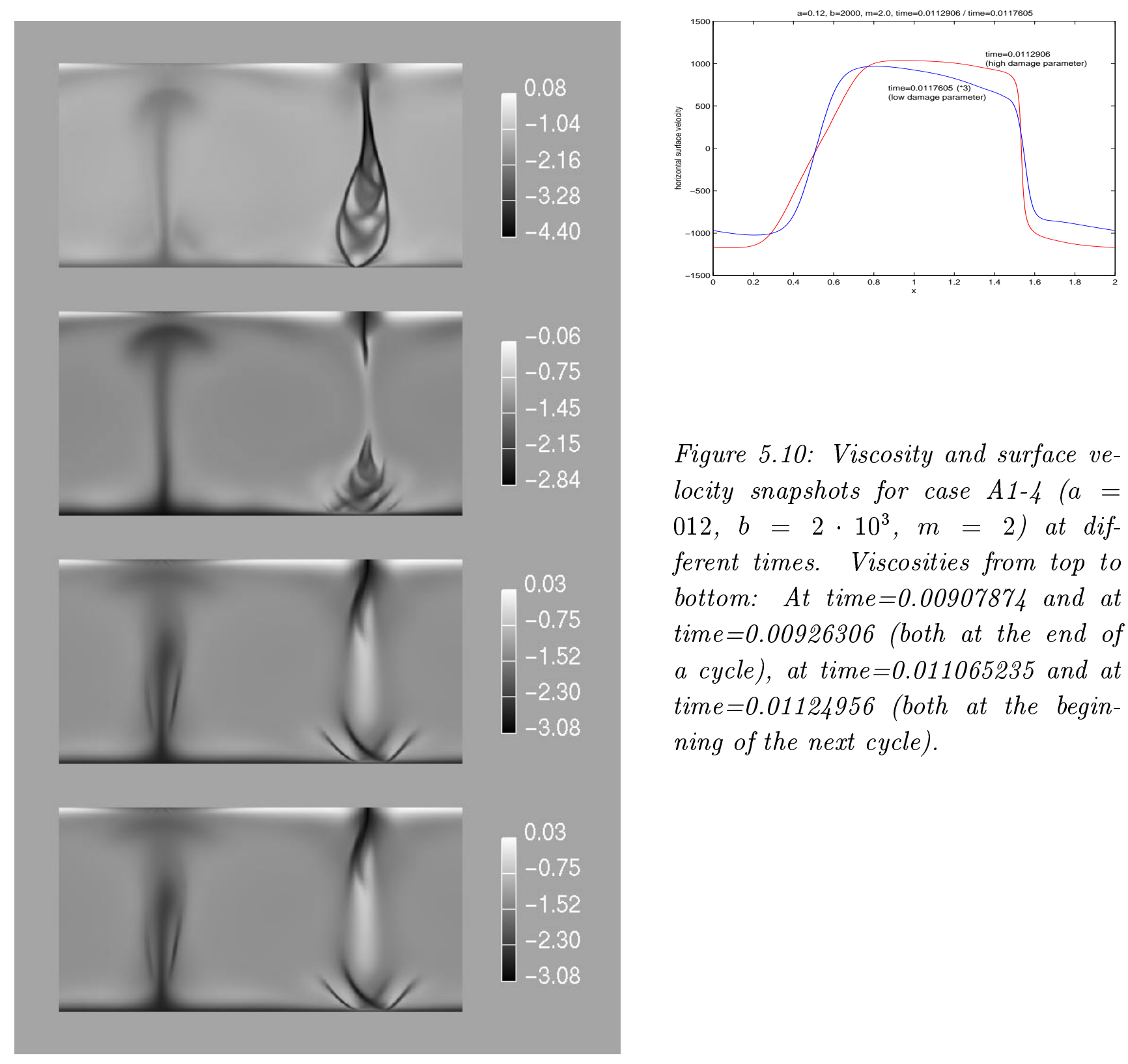

Figure 5.10: Viscosity and surface velocity snapshots for case A1-4 ( $a=$ $012, b=2 \cdot 10^{3}, m=2$ ) at different times. Viscosities from top to bottom: At time $=0.00907874$ and at time $=0.00926306$ (both at the end of a cycle), at time $=0.011065235$ and at time $=0.01124956$ (both at the beginning of the next cycle).

fast subduction and maybe $1 \%$ otherwise. It is significant that this kind of episodic behavior is completely different from what is observed for case A0-1; in particular, subduction always occurs here, although the velocity of the subducting material is variable. This case is in fact more comparable to case A0-3, although convection in A0-3 is less episodic. This is primarily because the viscosity is lower in the LVZs in case A0-3 and these zones are less focused so that the decoupling from the rest of the mantle is less effective. Secondly the weakening of the slab on the top of the box is less intense in A0-3 than in A1-4.

Although the increase of $a$ in A1-1 through A1-4 enhances the asymmetry of subduction, the convective behavior becomes episodic and the surface velocity inhomogeneous during the periods in where there is a broad LVZ above the upwelling.

For case A1-5 $(a=0.16)$ I get episodic behavior similar to that for case A1-4 $(a=0.12)$, 

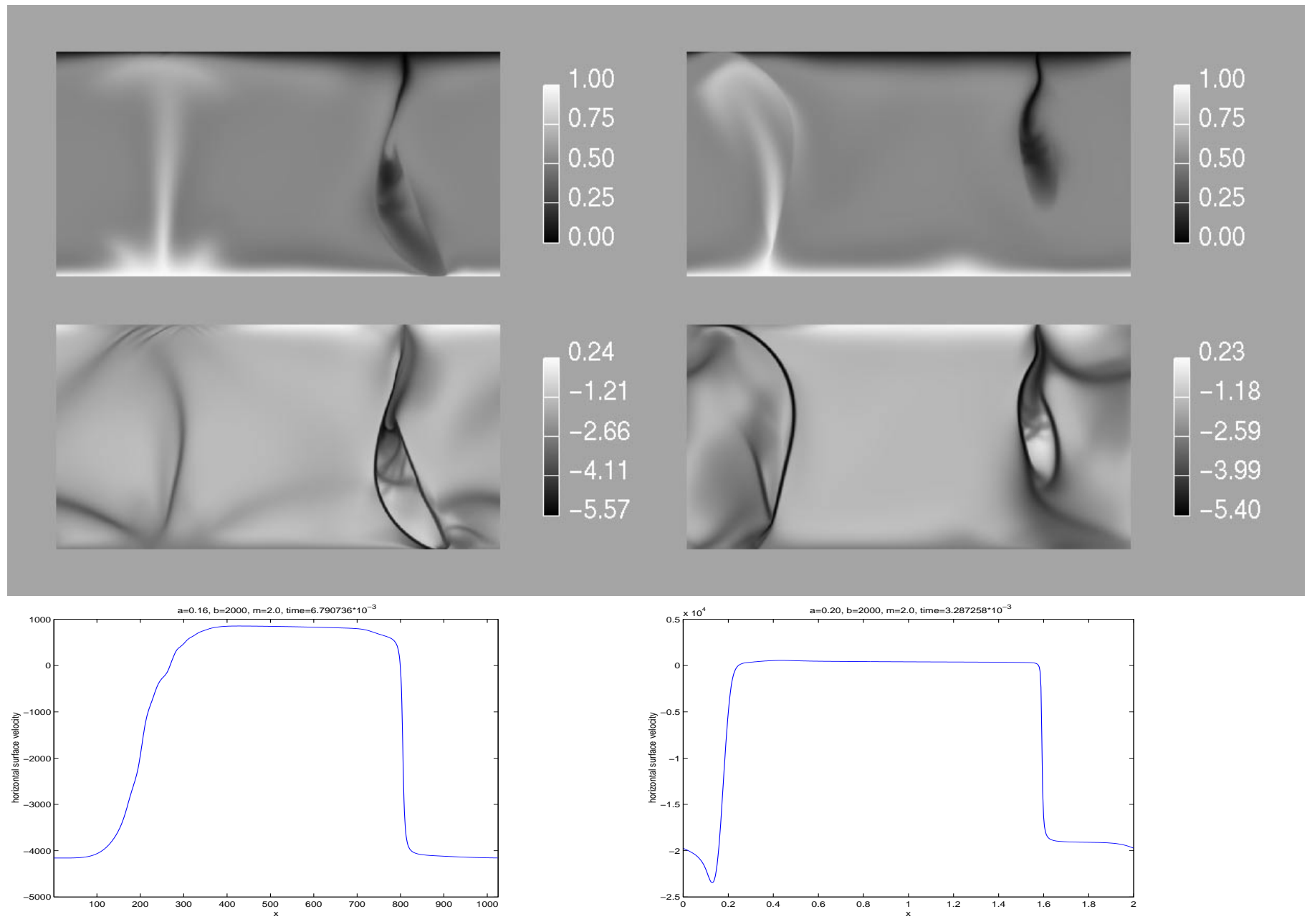

Figure 5.11: Temperature, viscosity and surface velocity snapshots during a fast subduction event for case A1-5 (left column) and A1-6 (right column)

except more extreme. The peaks in the root-mean-square velocity can reach values up to $10^{4}$ (which corresponds to $10 \mathrm{~cm} /$ year and is around seven times more than the value for A1-4), the maximum damage can come up to 200 (compared 100 for A1-4), the differences in the vigor of convection in the two different cells increase. It is significant that I see some focusing of the LVZs on the top and on one side of the upwelling at least during some of the episodic events (shown for the fourth event in figure 5.11 more significant for the second event). Therefore the surface velocity changes more abruptly above the upwelling than in case A1-4, although the LVZ above the upwelling is still extended.

When $a=0.2$ (case A1-6) the root-mean-square velocity increases to peak values around $2 \cdot 10^{4}$, and the maximum damage parameter increases to around 240 . The differences in the vigor of convection are clearly visible from figure 5.11. Compared to the fast movement of material in the "active" cell, the convection in the center cell is quite weak. Nevertheless the root-mean-square velocity within the center cell has approximately the same amount as in cases with much lower a (case A1-1 for example). Corresponding to this situation 
the asymmetry of the subduction is very high and mostly material from the "active" cell is subducted. In contrast to cases with lower $a$ values a significant asymmetry is also visible in the upwelling. The LVZ above the upwelling is much more focused now, than in the previous cases. Accordingly the changes in the surface velocity above the upwelling and the downwelling are both very sharp and the velocity through much of the region between them is very homogeneous.

\subsubsection{Series A2}

Our major problem in series A1 is the broad LVZ above the upwelling. To obtain more focused LVZs the $a$ and $b$ values for my new start case A2-4 (cases A2-1/2/3 are not presented here) are increased by a factor of five compared to case A1-1. A typical viscosity field and
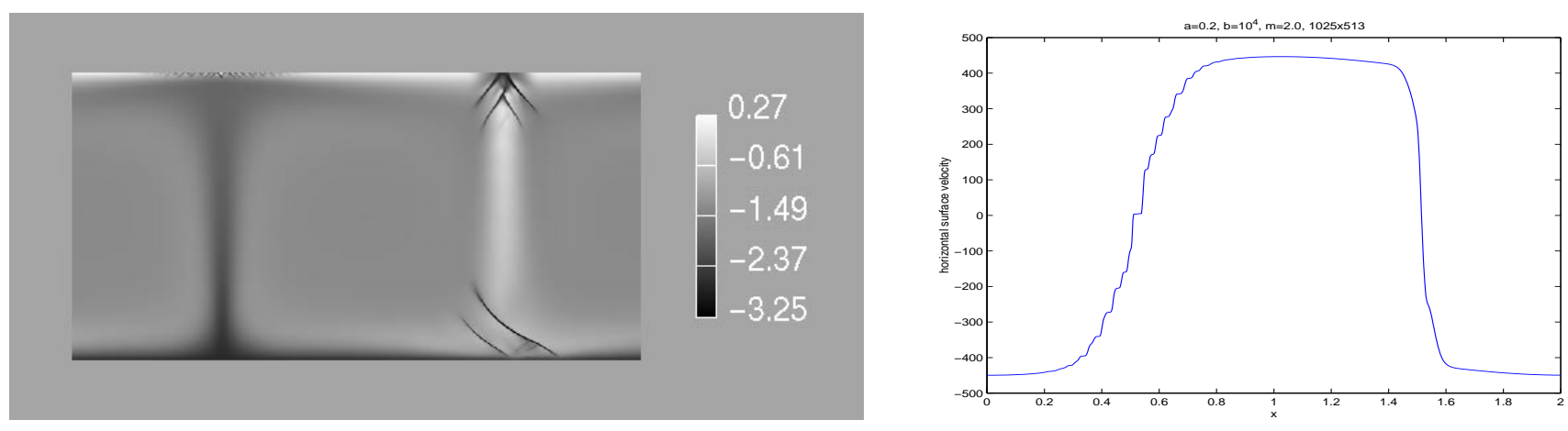

Figure 5.12: Steady state viscosity and surface velocity for case A2-4

the corresponding surface velocity for case A2-4 are shown in figure 5.12. The structure of the LVZs is already very similar to the case M0-6 ( $a$ and $b$ increased by another factor of five; see figure 5.5) except that the zones are broader and the viscosity is not that low, which enables me to perform those calculations with few resolution problems (small parts of the "v" structure directly above the upwelling are sampled only by three grid points).

I now increase $a$ to 0.4 (case A2-5) and 0.5 (case A2-6). A2-5 (not shown here) produces a similar behavior to A2-4 except that the temporal variation of the damage parameter field is a little higher.

For A2-6 I obtain episodic behavior again; as in case A1-4 I see a sudden increase in the values for the root-mean-square velocity and the damage parameter; moreover, convection is more vigorous in one cell than in the other one and a lens shaped LVZ coats the downwelling (see figure 5.13. A LVZ around the upwelling however is not visible. Two differences between cases A1-4 and A2-6 are significant: In case A1-4 the LVZ on the top of the downwelling is always nearly linear. Also the asymmetry develops a little deeper inside the subduction zone. However in case A2-6, the subduction asymmetry is clearly visible at the surface, too. The temperature field (figure 5.13) shows subduction with an angle of around 60 degrees to the surface. The second important feature is the LVZ above the upwelling. Although this zone 

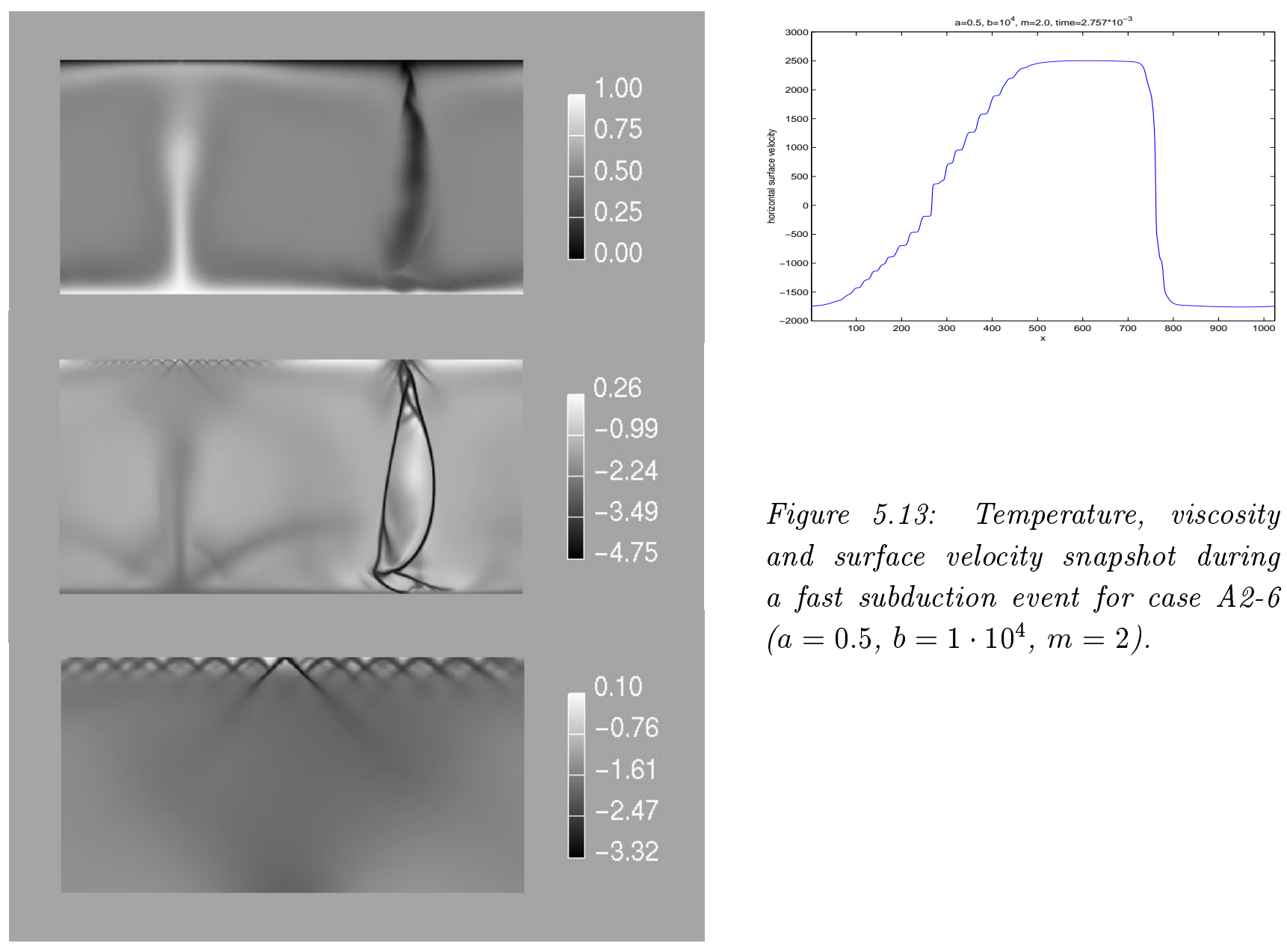

Figure 5.13: Temperature, viscosity and surface velocity snapshot during a fast subduction event for case A2-6 $\left(a=0.5, b=1 \cdot 10^{4}, m=2\right)$.

becomes broader in case A2-6 compared to case A2-4, the focused "v" shaped LVZs survive and the change in the surface velocity is not that homogeneous as it is in case A1-4 (figure 5.13). In fact the surface velocity changes in a lot of small little jumps and micro-plates are developed. A further increase of $a$ might be able to improve the plate-like behavior as in previous examples, unfortunately those calculations are computationally beyond our means.

Case A2-6 is important for another reason. I have shown before (series AB) that a simultaneous increase of $a$ and $b$ usually changes the structure and sharpness of LVZs but not the general convection pattern. In contrast a comparison between A1-3 and A2-6 demonstrates that a simultaneous increase of $a$ and $b$ can drastically change the convective regime, if the $a / b$ ratio is chosen close to that regime transition. However, the assumption that the general convective pattern in the box weakly depends on the absolute values of $a$ and $b$ remains a appropriate approximation at least for the parameter range I am investigating here.

\subsubsection{Series A3}

The parameters $a$ and $b$ can be further increased simultaneously if I decrease $m$ back to $m=1.5$. Starting from case A3-1 (identical to case M0-5) I first increase $a$ to 4.0 (case A3-2) 

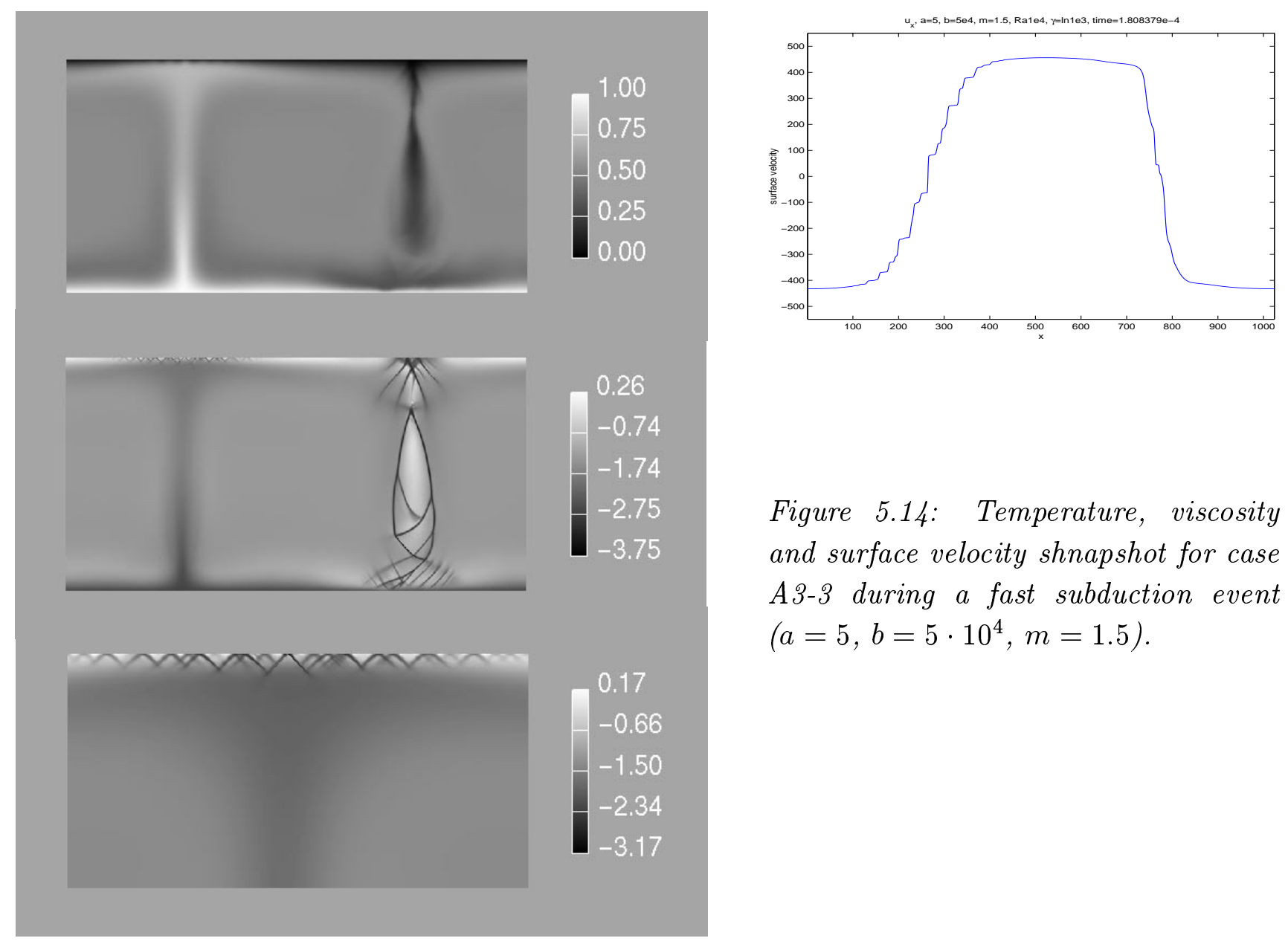

Figure 5.14: Temperature, viscosity and surface velocity shnapshot for case A3-3 during a fast subduction event $\left(a=5, b=5 \cdot 10^{4}, m=1.5\right)$.

and then to 5.0 (case A3-3). The case A3-2 gives us a time-dependent but non-episodic result similar to the starting case, the viscosity in the LVZs is lower and the zones are more extended. The result for case A3-3 (figure 5.14) is similar to case A2-6. Therefore the LVZs above the upwelling can be further focused (compared to case A2-6) and this focusing remains stable during the whole episodic convection. Thus the surface velocity changes in a more plate-like way here, as well.

\subsection{Summary of the results for bottom heated models}

In this section I summarize and organize my results according to the influence of the different model input parameters, and discuss how plate-like my simulations finally are.

\subsubsection{Influence of the parameter $a$}

To facilitate my discussion, I classify my results in terms of the parameter $a$ into different regimes. I choose this classification, because the variation of $a$ has the most significant 
influence on the convective system. For $b$ and $m$ fixed, at least four different regimes of convection can be distinguished:

Regime I: A calculation in this regime shows no significant differences to a calculation using only the corresponding temperature-dependent rheology. The parameter $a$ is relatively small and damage is not very significant.

Regime II: In this regime, episodic convection is obtained and damage influences the circulation only during episodes of strong subduction; $a$ is a little higher than in Regime I.

Regime III: Convection in Regime III either reaches a steady state or is weakly timedependent but with a stable convection pattern. A permanent influence of damage is visible; $a$ is further increased compared to Regime II.

Regime IV shows episodic behavior again. When a slab is sufficiently subducted the whole subducted part is coated in LVZs. This results in very fast downwellings, primarily because the subducting material is decoupled from the rest of the mantle. The episodicity itself results from the slab periodically detaching from the top of the box. $a$ is higher here than in Regime III.

An explicit specification of the $a$ values for the boundaries between the different regimes is not presented here, since these values depend on $b$ and $m$. The computational effort to calculate them for a significant number of $b$ 's and $m$ 's would be much too high. However, series A0 and A2 (tables 5.1 and 5.2)might give an idea about the boundaries for at least two values of $m$. Although the $b$ values are different in both series, the results are comparable, since for a fixed value of $m$ the $a / b$ values of the regime boundaries are only weakly variable in the parameter space I investigate here and can therefore assumed to be constant to good approximation.

\subsubsection{Influence of advection and $m$}

We have shown, that the advection of damage significantly influences the convective structures of my system. For low absolute values of $a$ and $b$ (high advection) the LVZ are linear, higher values of $a$ and $b$ produce "v" shaped LVZs and finally self-focusing of these zones $(m>1.0$ assumed). Moreover the time-dependence of the models increases with increasing $a$ and $b$ values.

Since the transition from non-self-focusing to self-focusing behavior is very important, I include it in my regime classification. The non-self-focusing part of for example Regime III is therefore called Subregime IIIa the self-focusing part Subregime IIIb. In fact, Regime III is the only regime, in which we observe both non-self-focusing and self-focusing behavior. Our calculations in Regime II always show non-self-focusing behavior, the ones in Regime IV show self-focusing behavior. Nevertheless the existence of a Subregime IVa or a Subregime IIb (temporary presence of self-focusing behavior) can not be excluded from my simulations. Following my results in series AB and M0 the boundary between Subregimes IIIa and IIIb is determined using the time averaged maximum of the second invariant of the strain rate tensor $\dot{\varepsilon}_{\text {calc }}$. Figure 5.15 shows $\dot{\varepsilon}_{\text {calc }}$ over the $a-m$ plane for a fixed $a / b=2 \cdot 10^{5}$ ratio. The 
curve including the black points is our approximation to the boundary between Subregimes IIIa (closer to the point of view) and IIIb in the $a-m$ plane. It was determined by using the fact that the maximum of the strain rate remains nearly constant throughout Subregime IIIa but increases suddenly upon entering Subregime IIIb. The explanation of the boundary curve between these Subregimes was in fact already given in my discussion of the influence of the parameter $m$ : An increase in $m$ acts in principal against the advection term in the damage parameter equation (see section "Series M1" for further discussion). If $a$ and $b$ are relatively large the advection term in equation (3.8) is less important also, thus a lower value of $m$ is sufficient to obtain self-focusing behavior. That the $\dot{\varepsilon}$-values for the transition between Subregimes IIIa and IIIb are much higher than expected from my simple analysis of the damage equation without the advection term (see section "Simple Analysis without Advection") demonstrates the significant influence of the advection of damage for convective structures (see section "Series AB" for extended discussion).

\subsubsection{Plate-like behavior}

Judging my regimes in terms of focused LVZs, homogeneous surface velocities and asymmetric subduction (plate-like behavior) I come to the following results:

(1) Regime I shows no plate-like behavior at all since no damage is present. The surface

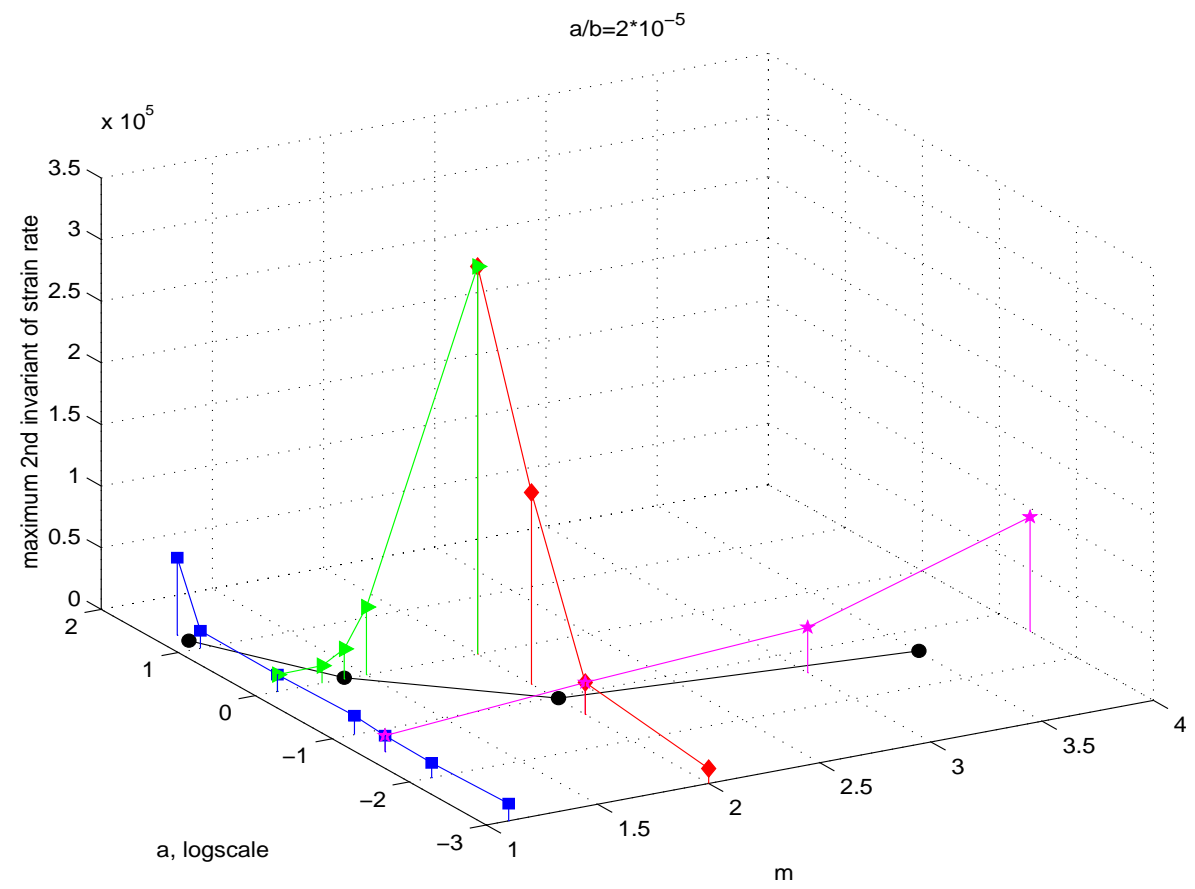

Figure 5.15: Illustration of the transition between Subregimes IIIa and IIIb. Plotted are the temporally averaged maximum values of the second strain rate invariant over the $a-m$ plane. $a / b=2 \cdot 10^{-5}$ is kept constant. The transition between Subregime IIIa (closer to the point of view) and IIIb is marked by the solid black line/circles in the $a-m$ plane 
velocity is nearly sinusoidal.

(2) Regime II shows plate-like tendencies only during episodes of strong subduction. Convection in this regime may be qualitatively comparable to the results of Trompert and Hansen (1998), who observed periods of strong subduction separated by periods with no subduction for calculations with a yield stress rheology.

(3) The LVZs in Subregime IIIa are present during the whole calculation, but with elongated and linear shapes. The surface velocity is usually homogeneous, but the transitions over the downwelling and upwelling are quite diffuse. This is comparable to simulations with common power law rheologies using positive and finite power law exponents (see for example Christensen (1984)). Keeping my simple analysis (see equation (3.11)) in mind, the similarity between damage and power law rheologies is not surprising, because their $\sigma(\dot{\varepsilon})$ curves are similar before $\dot{\varepsilon}_{s l}$ is reached (see figure 3.1). In Subregime IIIb, the LVZs are much more focused than in Regime IIIa, and are more or less "v" shaped. Such "v" structures are also found in studies of localization in compressive folding using power law rheologies with negative exponent (Montesi and Zuber 2001). The surface velocity is much more homogeneous, with a few jumps over the upwelling (micro-plates) and one or two big jumps over the downwelling. The asymmetry of the subduction is usually not very developed.

(4) The appeal of Regime IV is that subduction is usually asymmetric. However, the subduction process is also episodic. This kind of episodicity is not the same as in Regime II, because here subduction never really stops, although the slab breaks off from the surface. The differences in the surface velocities therefore occur because of the different pull of the already subducted material on the rest of the lithosphere. During episodes of strong slab pull the surface velocity is usually much too high. A non-dimensional velocity of 1000 in my calculations roughly corresponds to a real velocity of about $1 \mathrm{~cm} /$ year. So, compared to the velocity of Earth's plates, non-dimensional velocities much higher than, say, $10^{4}$ are unrealistic. In addition, the LVZ above the upwelling can become very diffuse during these episodes of strong slab pull, causing a very un-ridge-like velocity change right there. This problem mostly occurs for relatively low values for $b$, when Regime IV is entered from Subregime IIIa. 


\section{Chapter 6}

\section{Internally heated models I}

Since the Earth's mantle is probably around $80 \%$ heated from within, models using the internal heating mode are discussed now. The Rayleigh number and the temperaturedependence of viscosity are chosen to produce low viscosity / sluggish lid convection without the presence of damage.

\subsection{Introduction}

Some general questions and expectations about internally heated models are addressed.

\subsection{Case ISL-1}

Presents the reference case for this chapter.

\section{3 - 6.5 Case ISL2 - Case ISL4}

Investigate changes in the convection pattern due to modifications of the box width, $a$ and $a, b$.

\subsection{Discussion and Conclusion}

Summarizes this chapter's results with respect to the questions addressed in the Introduction. 


\subsection{Introduction}

The main difference between the bottom and the internally heated models is that the later ones usually do not show any localized plume-like upwellings. For my attempts to improve plate-like behavior in damage-dependent models, this can have two consequences:

(1) Asymmetric subduction zones might be more easily obtained than in bottom heated models since no stable two cell convection develops.

(2) Focused divergent boundaries between two plates might be more difficult to create since no plume-like upwelling is present to localize stress in the lithosphere.

Based on these expectations, I investigate the following questions in this chapter:

- Is it possible to obtain localized passive spreading zones for internally heated models?

- Can asymmetry of subduction zones be more easily obtained for internally heated systems than for bottom heated models?

- Can these results for internally heated models be classified into the four convective regimes developed in the last chapter for bottom heated models?

- Does the convection tend to become episodic again with increasing $a$ ?

For all the models in this chapter the Rayleigh number defined at the box surface is fixed to $R a=10^{5}$, the temperature dependence of the viscosity is $\gamma=\ln 10^{8}$ and the non-dimensional rate of internal heating is $q=1$. For pure temperature-dependent viscosity these parameters would produce a steady state close to the boundary between the Low Viscosity Contrast and the Sluggish Lid Regime. The exponent of the damage in the viscosity equation 3.7 is $m=2.0$.

\begin{tabular}{c|c|c|cc|c} 
Series & Case & Aspect Ratio & $a$ & $b$ & Regime \\
\hline \multirow{3}{*}{ ISL } & 1 & 2 & 0.100 & $5.00 \cdot 10^{4}$ & VDR II - HDR \\
& 2 & 4 & 0.100 & $5.00 \cdot 10^{4}$ & VDR II \\
& 3 & 2 & 0.300 & $5.00 \cdot 10^{4}$ & VDR II - VDR IV \\
& 4 & 2 & 0.025 & $1.25 \cdot 10^{4}$ & VDR II - HDR
\end{tabular}

Table 6.1: An overview of the parameters used in sections 6.2 - 6.5 .

\subsection{Case ISL-1}

The reference case ISL-1 (see table 6.1) uses $a=0.1$ and $b=5 \cdot 10^{4}$. The initial conditions for temperature and damage are taken from an analogous calculation with $m=1.1$. 


\subsubsection{Description of the convection pattern}

Figures 6.1 and 6.2 show the root-mean-square (rms) velocity and the maximum damage parameter versus time. Initially, the system is episodic and periods of high rms-velocities and high damage follow periods of very low rms-velocities and damage. Later on, approximately around a time of 0.03 the episodicity decreases and the convection becomes more regular. However, I focus on the description of the episodic part first. The further evolution of the system will be discussed next.

Snapshots of the viscosity field (figure 6.7) demonstrate the reason for the initial episodic convection pattern: After a "quiet" period - basically without subduction - a couple of lithospheric instabilities develop (four non-equidistant ones for the first cycle, see figure 6.7a). While most of these instabilities temporarily stop to grow one further increases and finally becomes a subduction zone, with significant damage above the downwelling (figures 6.7a and b). Approximately in the middle between the subduction and the adjacent instabilities, additional LVZs - passive spreading centers - develop (figure 6.7c left side). The surface material moves very uniformly between the subduction zone and the new passive spreading centers, its surface velocity (although highly variable) is in the order of some $\mathrm{cm} /$ year. The two plates at a subduction zone can move with different velocities, which seem to depend on their length at the time of their formation (compare the surface velocities for viscosity fields $g$ and $\mathrm{j}$ in figure 6.8 ). In contrast the plates penetrate into the mantle almost vertically. As the subduction proceeds the passive spreading centers become broader and move towards the subduction zone. Subduction stops when the spreading centers arrive at the downwelling. However, no passive spreading center vanishes into the

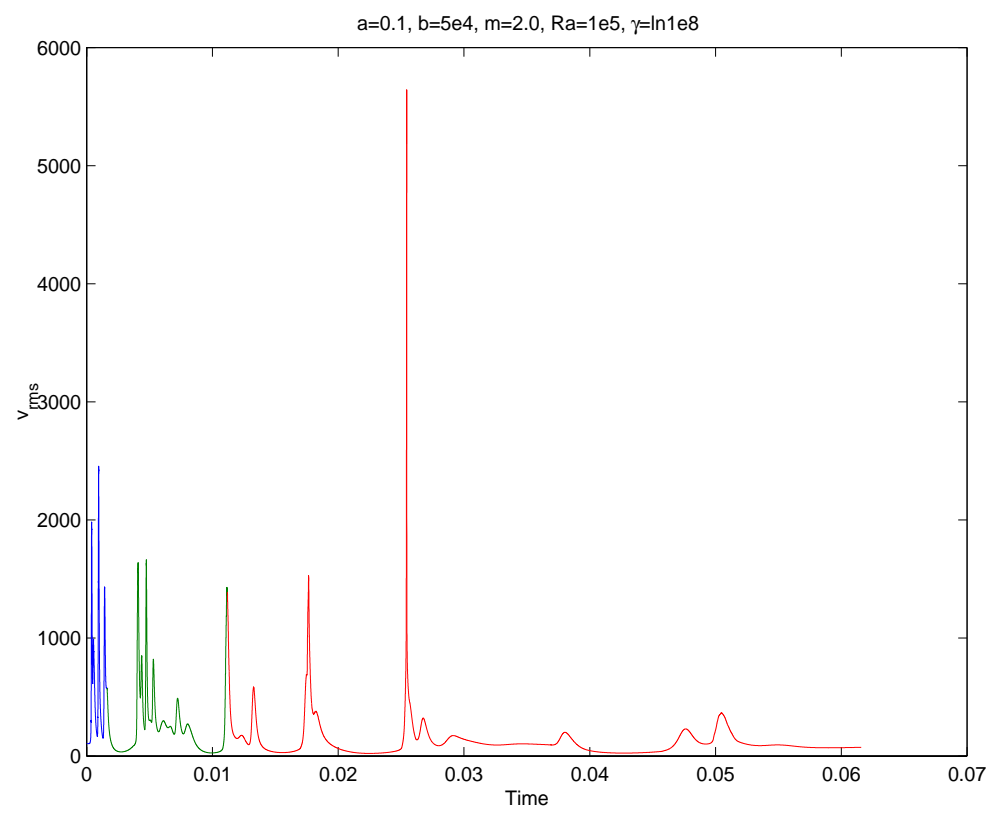

Figure 6.1: Root-mean-square velocity versus time for case ISL-1 


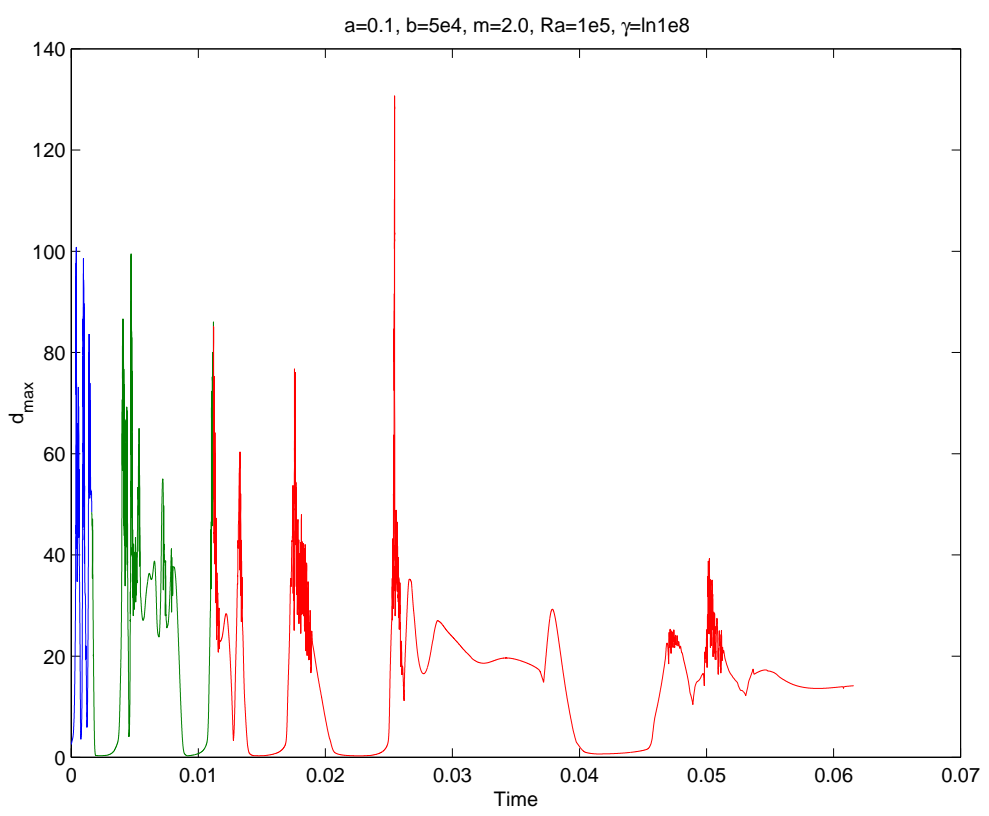

Figure 6.2: Maximum damage versus time for case ISL-1

mantle. It either become a spreading centers for the next developing downwelling process (spreading center on the left side of figures $6.7 \mathrm{c}, \mathrm{d}, \mathrm{e}$ ) or it simply heals (right spreading center in figure $6.7 \mathrm{~d}$ ). When all initial lithospheric instabilities are subducted, not enough cold and dense material is left to initiate a new subduction. Thus, the vertical movement of material nearly stops and passive spreading centers vanish (figure $6.7 \mathrm{~g}$ ). When the thermal

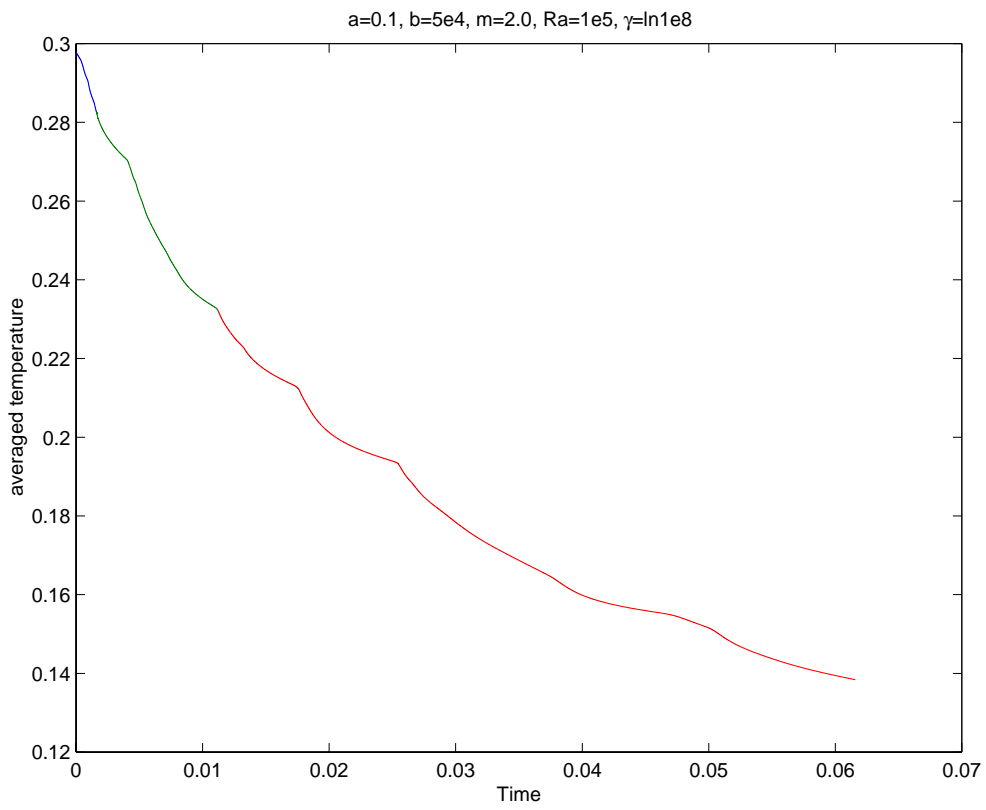

Figure 6.3: Averaged temperature in the box versus time for case ISL-1 


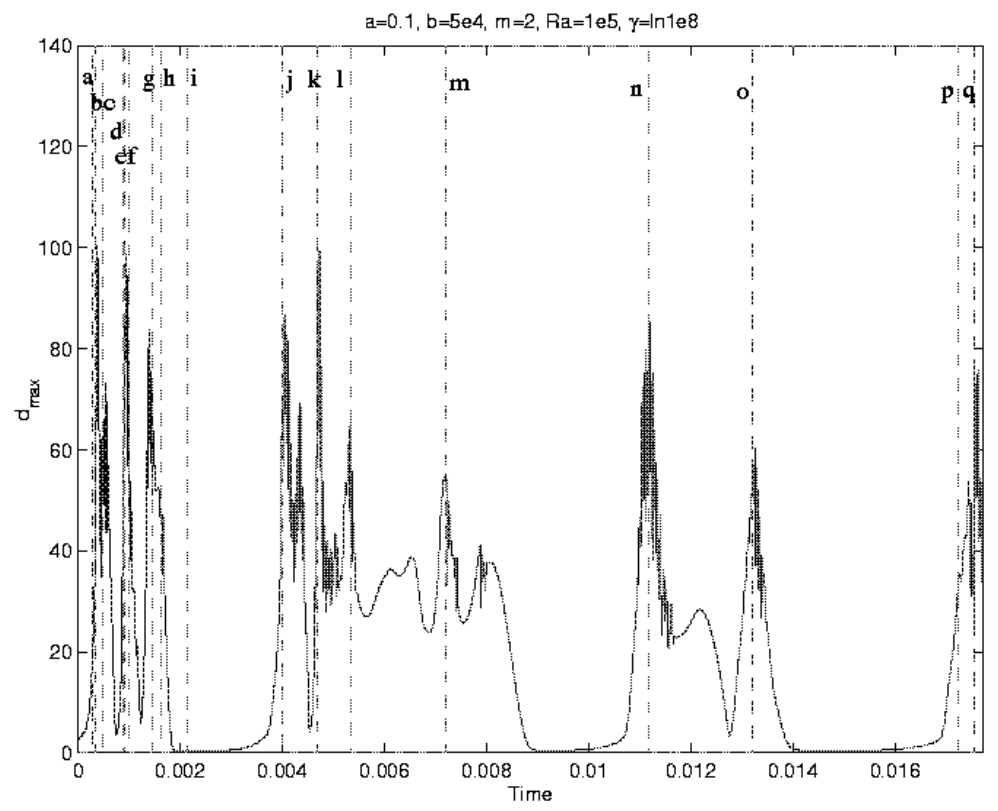

Figure 6.4: Maximum damage versus time for case ISL-1 Letters correspond to the letters of the viscosity snapshots in figure 6. $\%$.

boundary layer is grown up again, a new cycle starts.

However, the convection pattern in this example is slowly evolving into more continuous structures. The influence of damage is always present (Figure 6.2), but only above the downwelling. Passive spreading does not occur any more (Figure 6.7n). Except for an abrupt

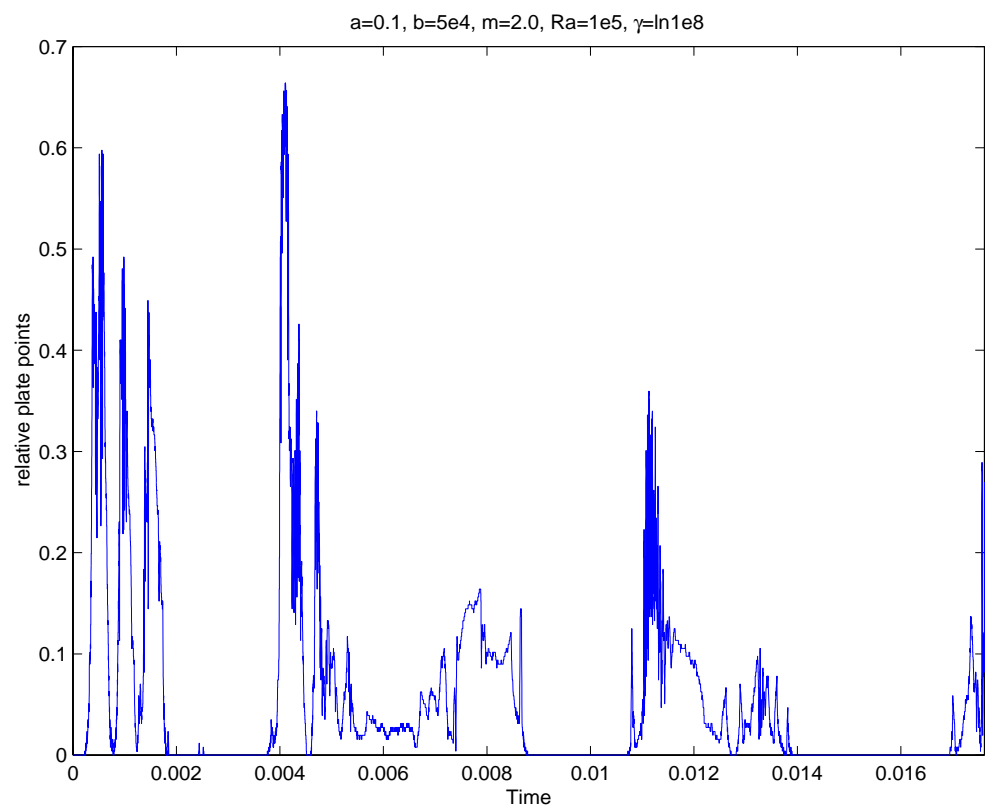

Figure 6.5: Relative number of plate points versus time for case ISL-1 


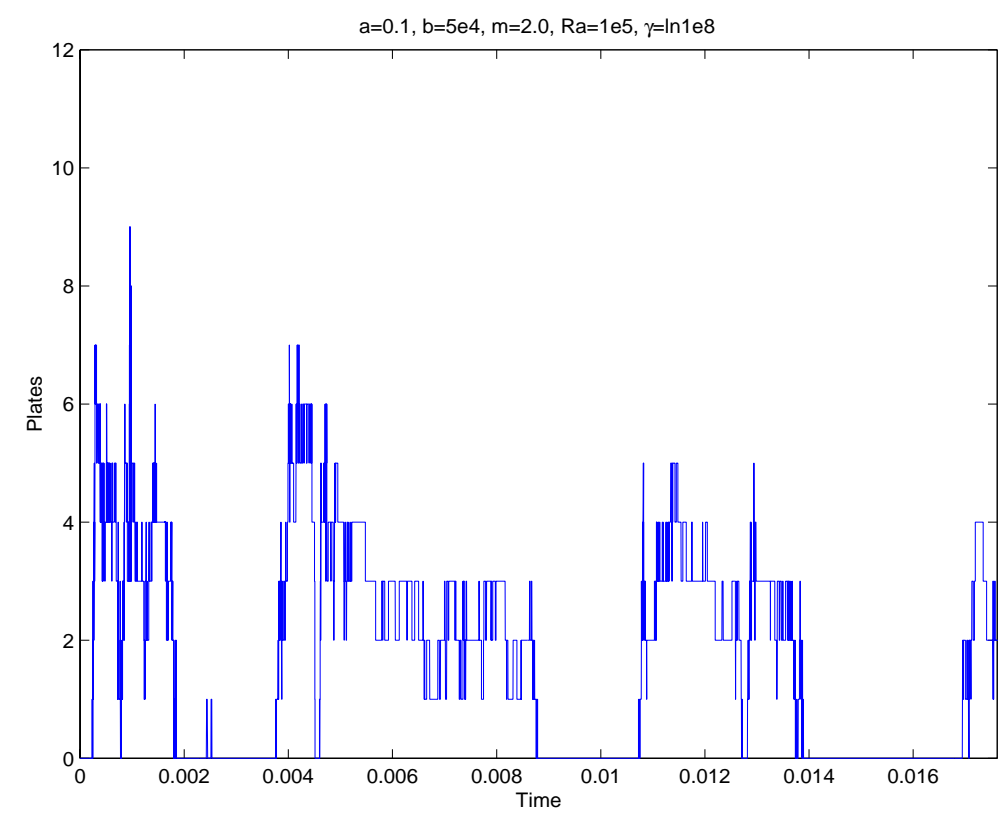

Figure 6.6: Number of plates versus time for case ISL-1

change above the downwelling, the surface velocity varies very uniformly. A comparison between the statistical steady state viscosity field for this calculation (Figure 6.7n) and the steady state viscosity field for the analogous calculation with $d \equiv 1$ (figure 6.9) shows, that both the fields are nearly identical except for the LVZ above the downwelling.

The reason for the slow change in the convective structure is that the simulation is initiated with a temperature significantly higher than the final steady state temperature. Thus, the temperature in the system decreases during the calculation (Figure 6.3).

The classification of this case into the regimes I defined for bottom heated cases is difficult. At the beginning the convection shows all the features of regime II, especially the episodic influence of damage. However, in the fourth cycle I observe a lense-shaped LVZ around the downwelling, which is typical for regime IV. And finally, a non-episodic convection with permanent damage influence is developed, as in regime III. Thus the classification scheme developed in the last section needs some modifications for internally heated systems. I discuss this point in detail at the end of this section.

\subsubsection{Summary of the observations}

- Initially the model shows episodic convection, while more regular behavior is obtained later on.

- The transition from the episodic to the regular convective pattern is caused by a significant decrease of the temperature in the system. 
a

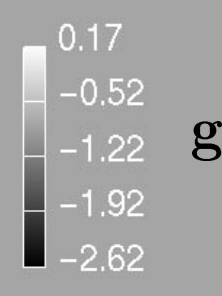

0.10
-0.77
-1.64
-2.51
-3.38

b
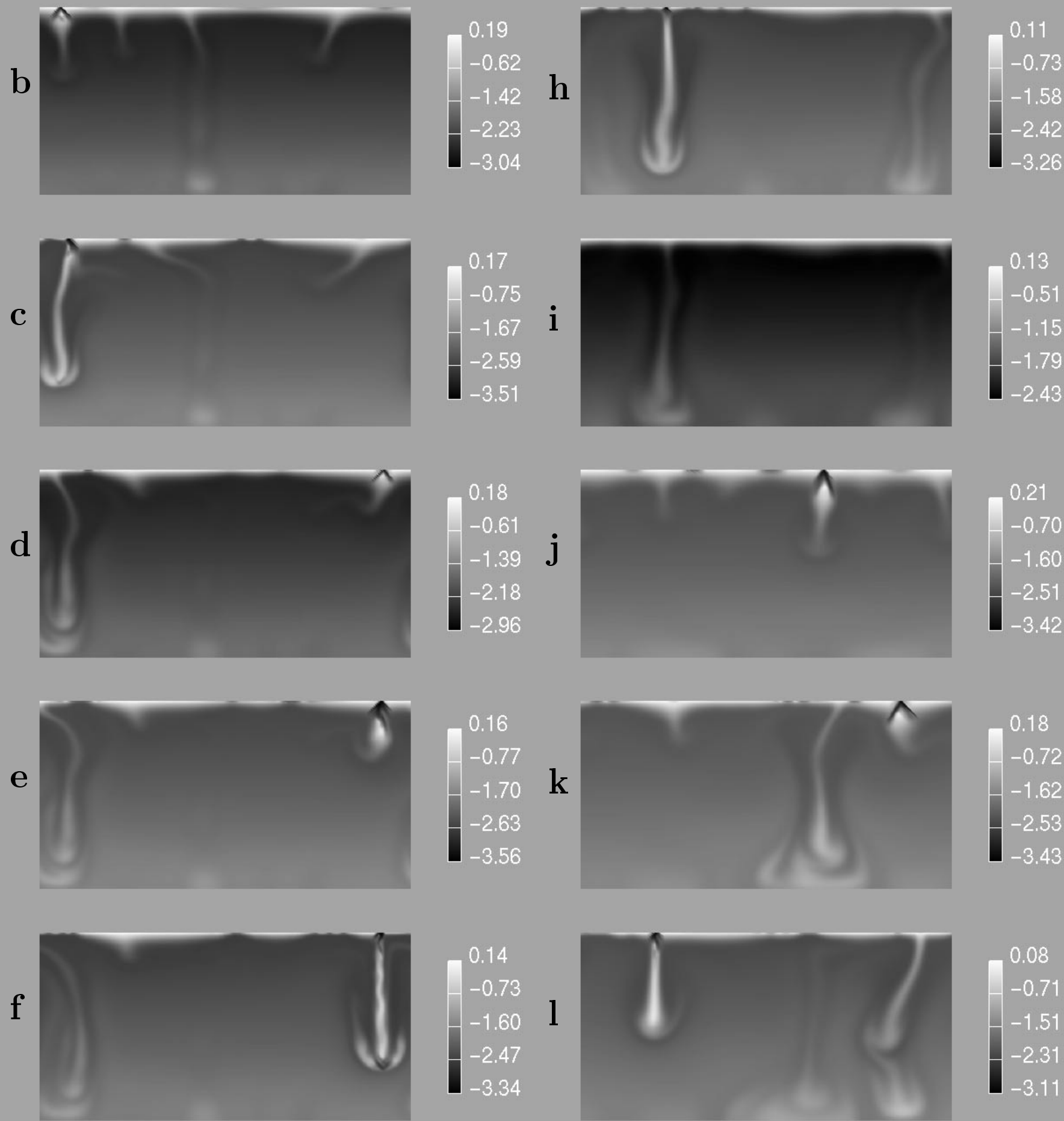


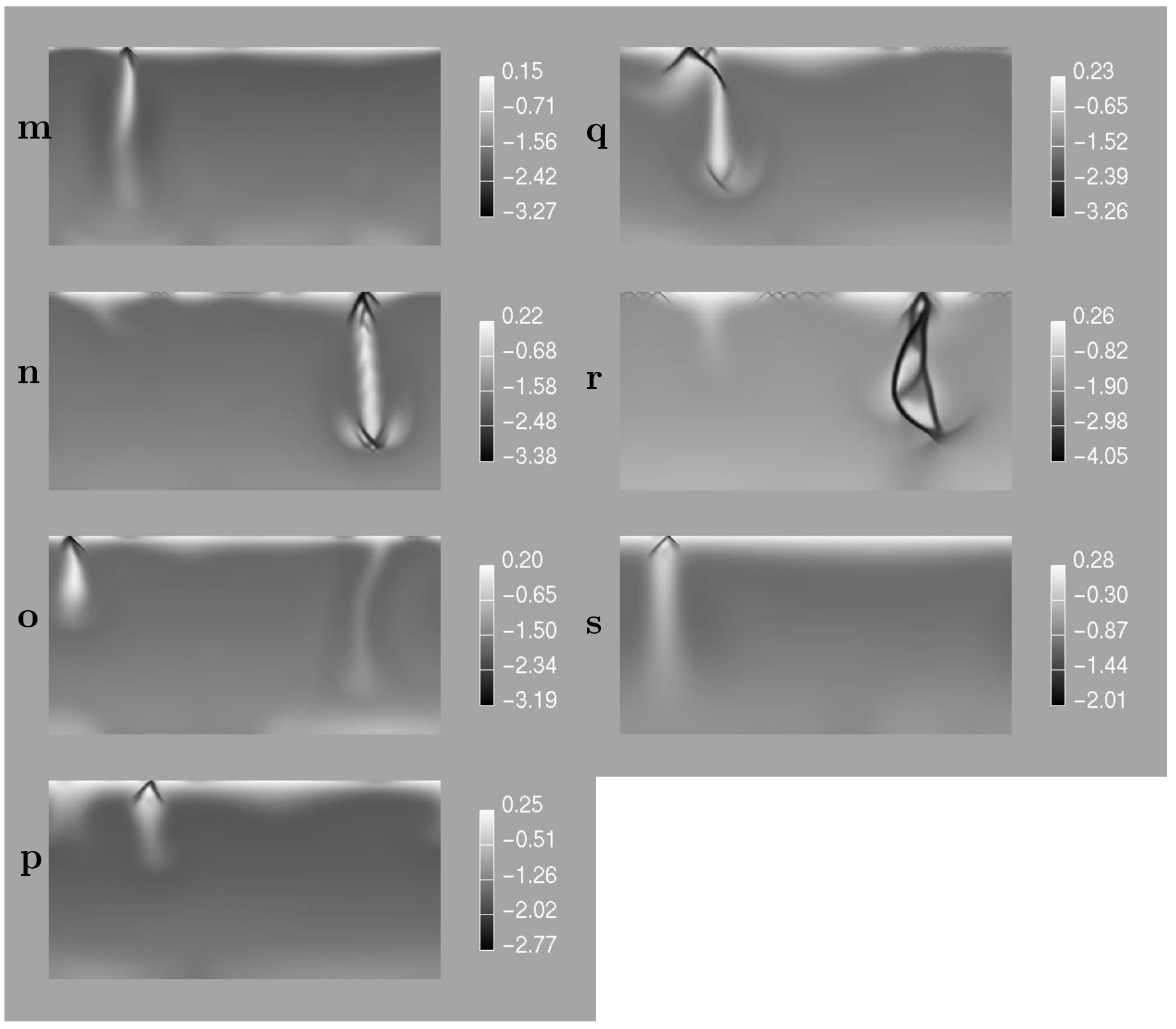

Figure 6.7: Viscosity snapshots for case ISL-1 

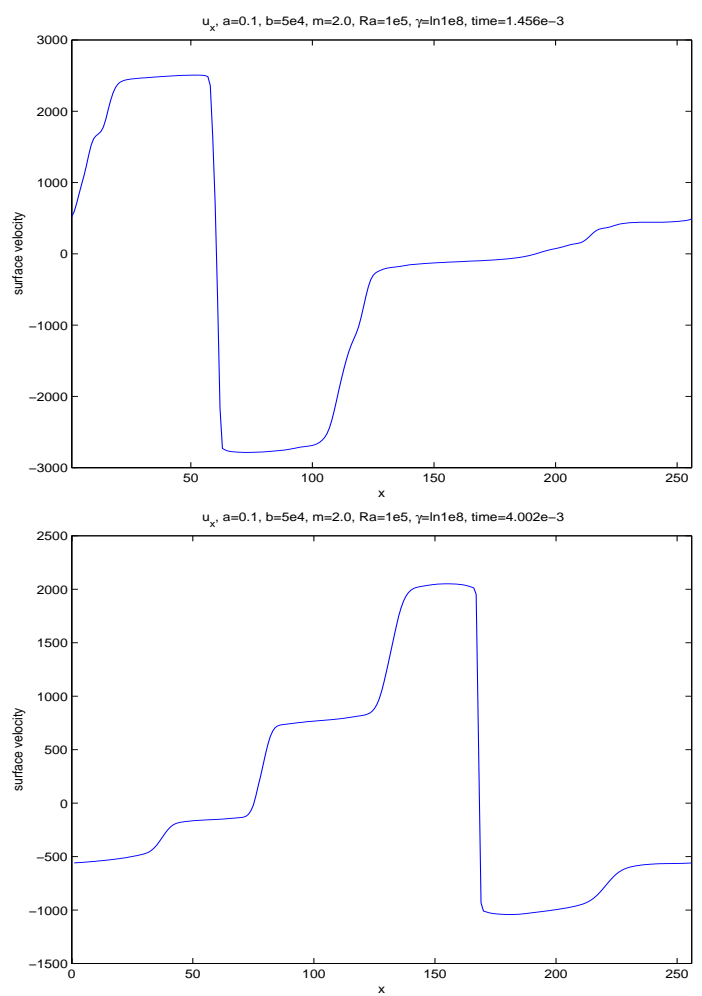

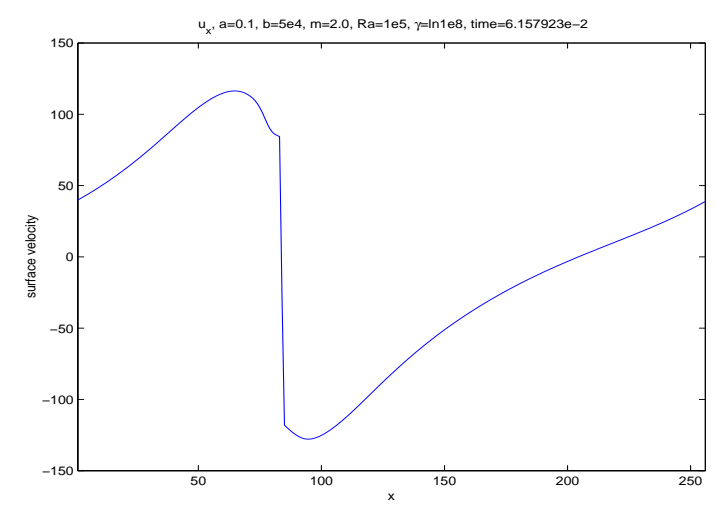

Figure 6.8: Surface velocities for case ISL-1 $\left(a=0.1, b=5 \cdot 10^{4}, m=\right.$ 2.0, $\left.R a=10^{5}, \gamma=\ln 10^{8}\right)$, corresponding to the viscosity fields " $g ", " j "$ (left) and "s" (right).

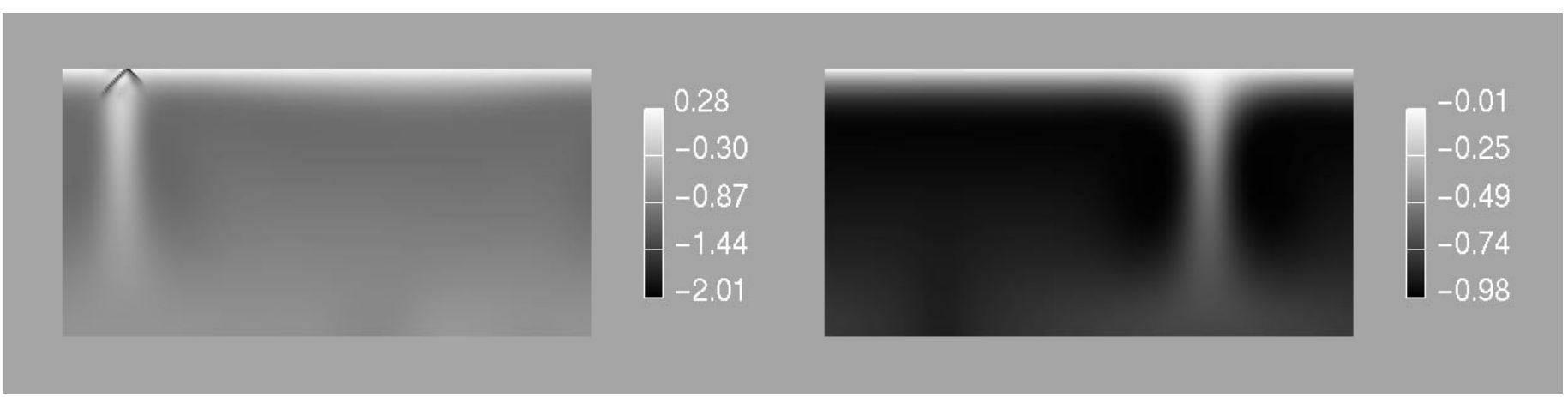

Figure 6.9: Comparison between the statistical steady state for case ISL-1 $(a=0.1, b=$ $5 \cdot 10^{4}, m=2.0, R a=10^{5}, \gamma=\ln 10^{8}$, top frame) and the steady state for the corresponding pure temperature dependent calculation (bottom frame). 
- At the beginning of the calculation localized passive spreading is observed during active episodes and the homogeneity and magnitude of the surface velocity are satisfactory while passive spreading is present. No passive spreading is obtained after the episodic part and the surface velocity changes very uniformly.

- The velocity of a newly developed plate depends on its length. Since two plates at a subduction zone do not necessarily have the same length, asymmetries in the surface velocities are obtained.

- The penetration of the lithosphere into the mantle occurs vertically.

- The maximum plate size in the episodic part (approx. $1000 \mathrm{~km}$ ) is significantly smaller, than the typical plate size observed on the Earth (several $1000 \mathrm{~km}$ ).

- Significant subduction of the lithosphere does not occur simultaneously in two regions of the box.

- The classification of this model into one of the regimes discussed in the last chapter is not possible. Features from different regimes are observed.

\subsubsection{Plate-like behavior, problems and their possible solutions}

How successful is this model in terms of plate-like behavior, where are its problems and how might these be resolved?

Since this model is clearly a very non-plate-like at the end of the calculation I focus on its beginning in this discussion and in all of the subsequent cases. Nevertheless the last part provides an important result: Passive spreading is not a self-evident feature in internally heated systems with permanent damage influence.

The episodic part of the case ISL-1 shows different plate-like aspects, for example, passive spreading, homogeneous surface velocities (during active periods) and asymmetric plate movement. Its problems are ...

(1) ... the "quiet" periods during the calculation. Since no subduction is present there are no LVZs above the downwelling and no passive spreading centers and therefore the convective behavior is very non-plate-like.

(2) ... the constant subduction angle of 90 degrees.

(3) ... the small size of plates.

One simple possibility to resolve the first problem might be to increase the box width. At the beginning of a cycle in the episodic part of the case ISL-1 different lithospheric instabilities develop, which subduct one after another. When the last one is subducted, the lithosphere at the position of the first one is not grown enough to form a new instability again. If the rule "only one subduction at the same time" holds in a wider box too, then a new cycle of subduction may start immediately after the old one is finished. 
The last chapter demonstrated the increase in the asymmetry of subduction with increasing damage source parameter $a$. This may be a relevant result also for the asymmetry in internally heated cases (see problem (2)).

A solution for the third problem could be to increase the temperature-dependence of the viscosity. However, this possibility will be discussed in other examples later.

\subsection{Case ISL-2}

Case ISL-2 uses the same parameters as case ISL-1, but with an extended aspect ratio of the computational domain. For initial conditions for temperature and damage, I use combinations from the fields corresponding to figure $6.7 \mathrm{~d}$ and $6.7 \mathrm{~g}$.

The snapshots of the viscosity field during the calculation (figure 6.13) show that the rule "just one active subduction zone at the same time" from case ISL-1 remains a good approximation here and that the lithosphere in passive areas of the surface has enough time to grow and become unstable, before subduction in active areas stops. In contrast to case ISL-1 I, thus, obtain a nearly permanent cycle of subduction without long quiet episodes (compare figures 6.4 and 6.10). As in the active parts of case ISL-1, uniform surface velocity depends on the existence of passive spreading centers which are related to a high damage parameter. The relative number of plate points is therefore also less episodic, than in case ISL-1, and a little higher. The plate size is not increased. Figure 6.12 shows, that the number of plates in this case is approximately twice as large as in case ISL-1 (figure 6.6).

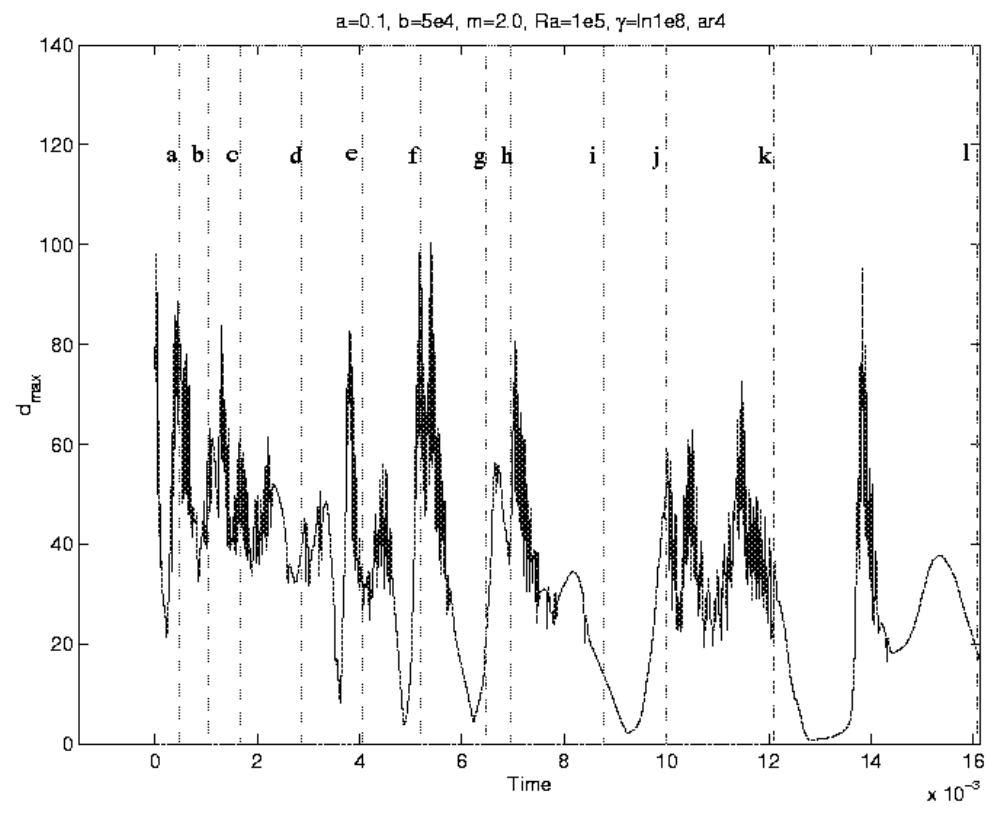

Figure 6.10: Maximum damage versus Time for case ISL-2 


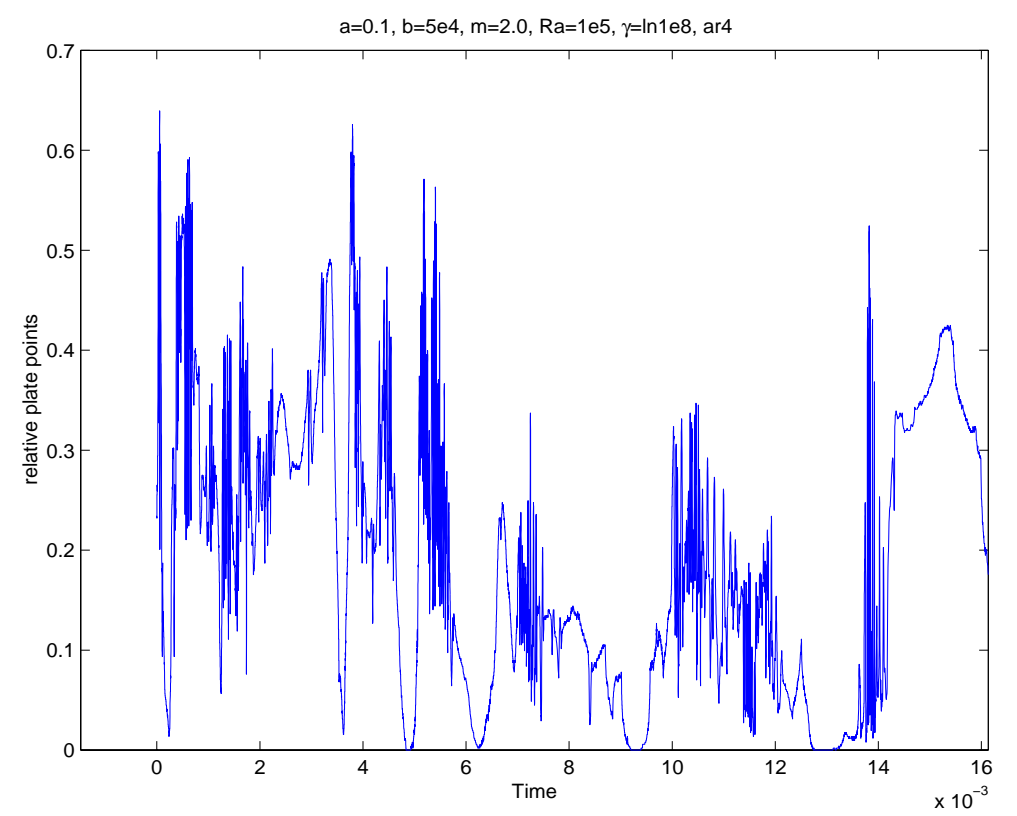

Figure 6.11: Relative number of plate points versus Time for case ISL-2

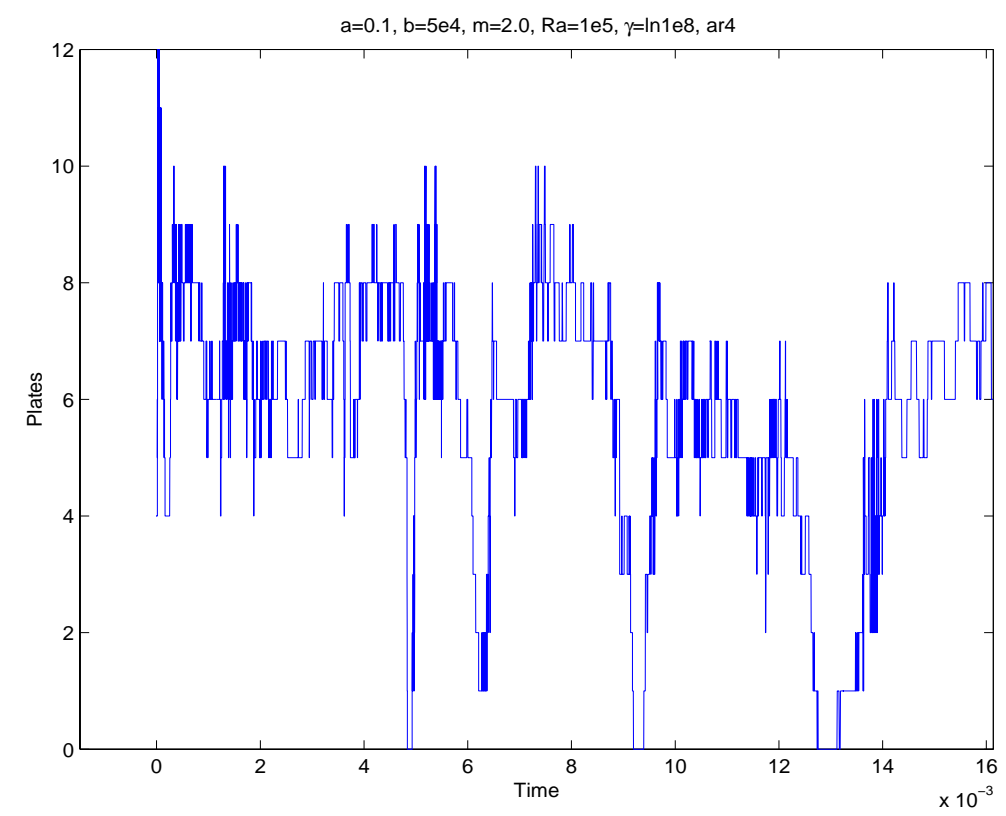

Figure 6.12: Averaged temperature in the box versus Time for case ISL-2 


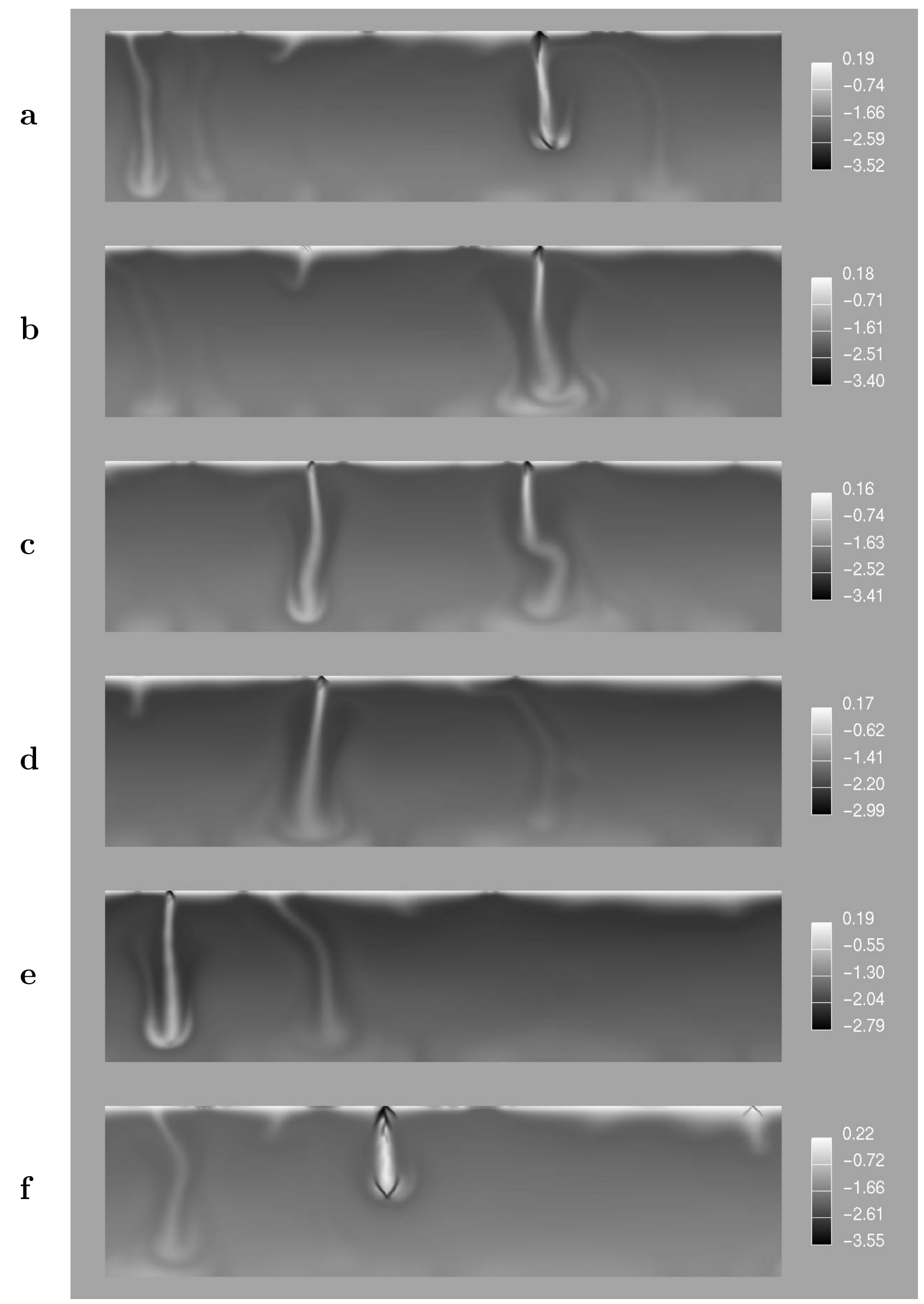




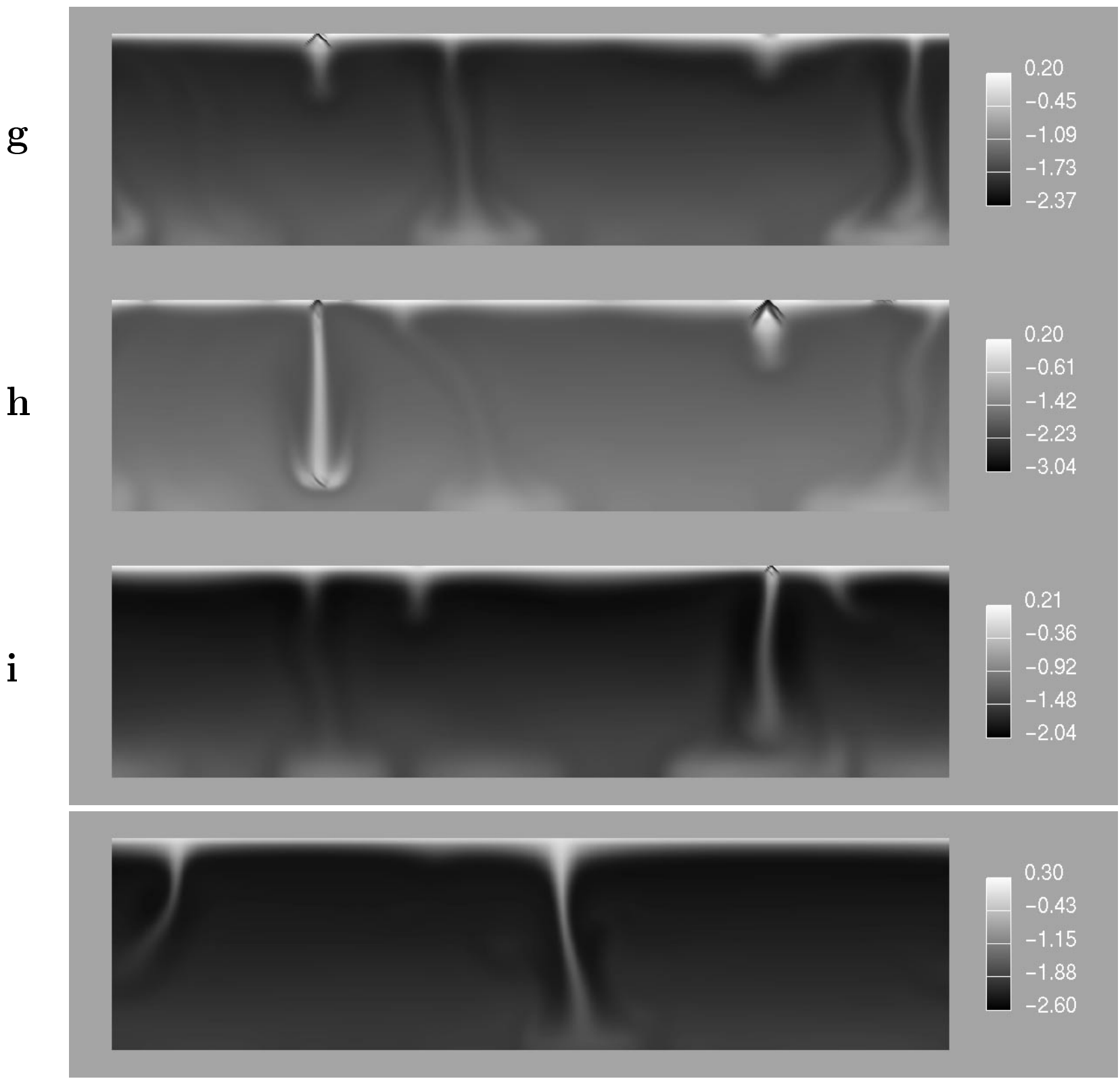

Figure 6.13: Viscosity snapshots for case ISL-2 The last frame shows a snapshot from the corresponding calculation with $d \equiv 1$ at a time of 0.006 . 

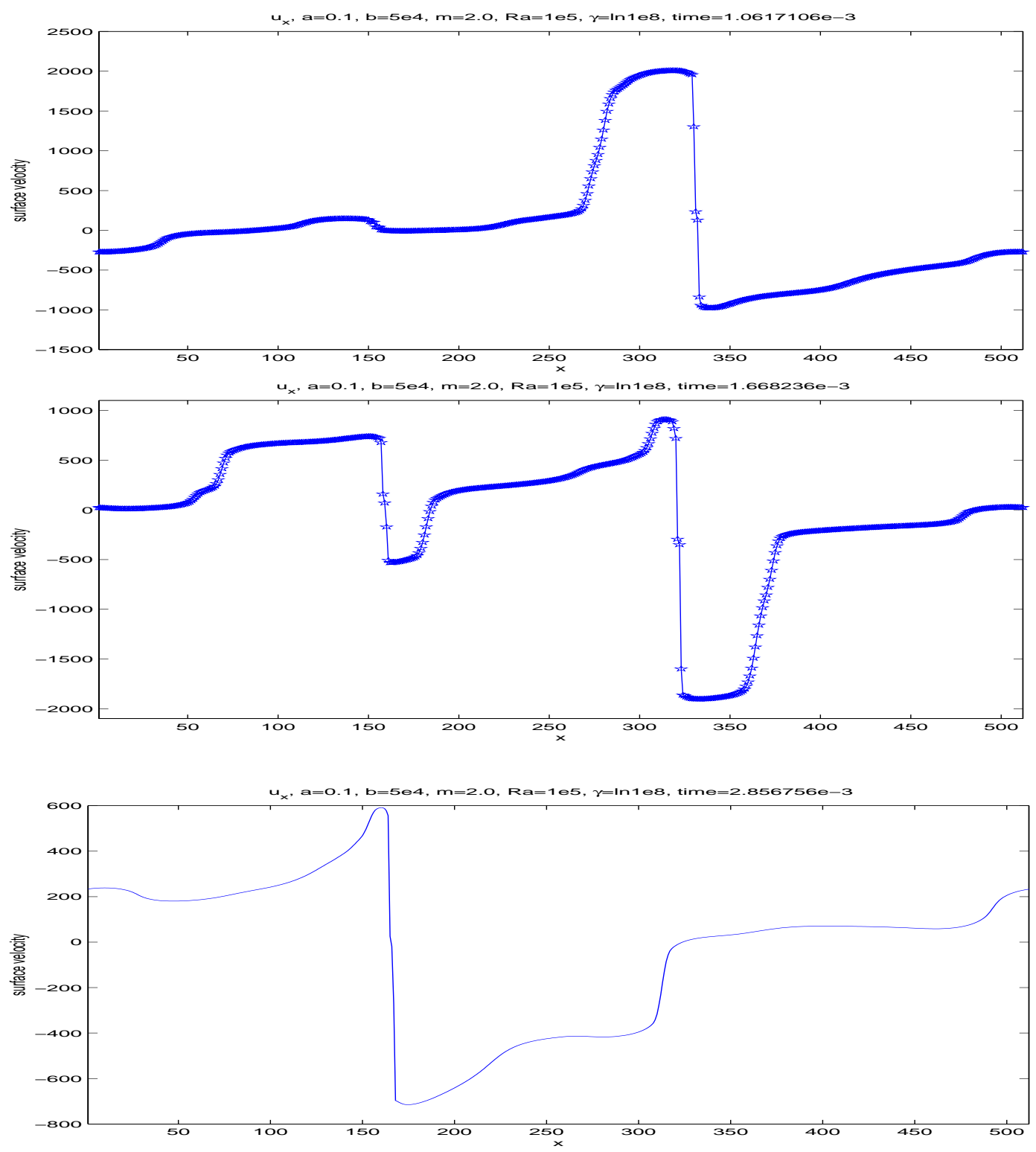

Figure 6.14: Surface velocities for case ISL-2 corresponding to the viscosity fields "b", "c" and "d". 
In summary, two things can be learned from this case:

(1) The existence of subduction-free episodes in case ISL-1 is at least in part an artifact caused by the width of the box.

(2) Although the lithosphere is broken into several pieces the large scale convection in the box has usually only two convection cells ("just one active subduction zone at the same time" - rule). This is an important difference from the analogous simulation with purely temperature-dependent viscosity, where a stable four cell convective structure develops shortly (time $=0.002$ ) after the start of the simulation with the same initial conditions (see last frame in figure 6.13). For a more viscous lithosphere the damage-dependent rheology may therefore promote the evolution of large plates.

\subsection{Case ISL-3}

This case is motivated from the last chapter's result that increasing the source parameter $a$ in the damage equation increases the asymmetry of subduction, although it also tends to make the convection highly episodic.

The parameters here are therefore same as in case ISL-1 except for the size of the damage source parameter $a$ which is increased to $a=0.3$. As initial conditions the temperature and damage fields corresponding to figure $6.7 \mathrm{f}$ are used. Although this calculation had to be stopped at a very promising point because of numerical difficulties, I present it to draw a more complete picture of internally heated cases.

Three aspects of the evolution in this case are significantly different from case ISL-1:

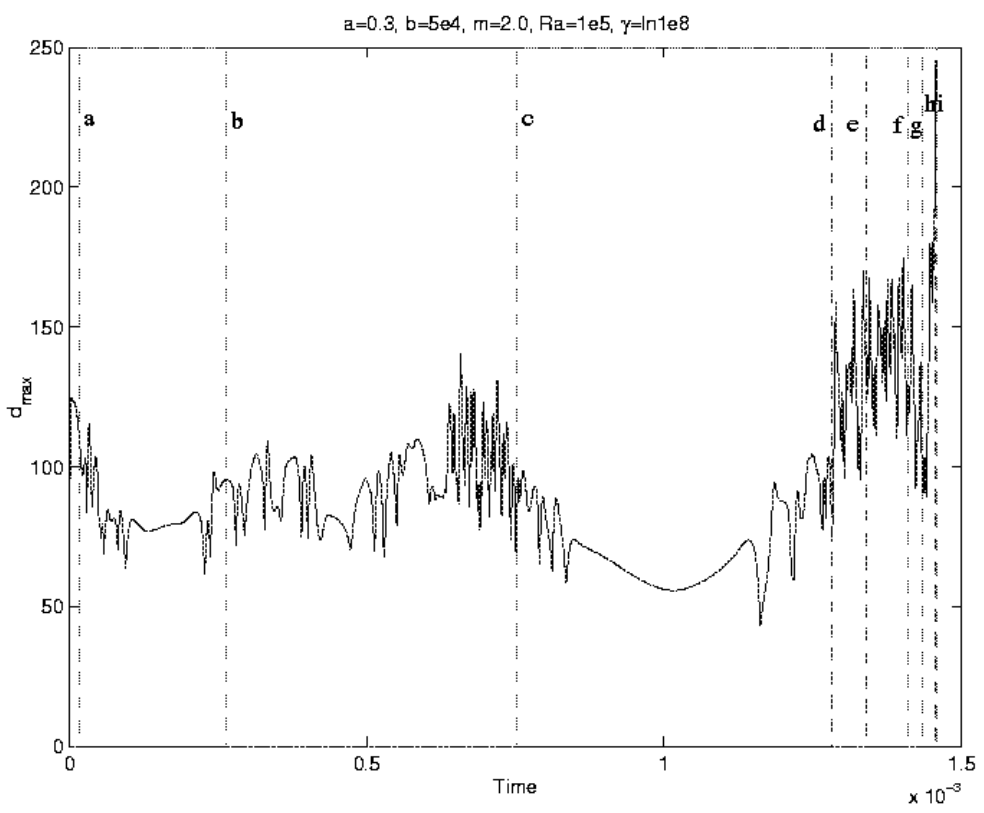

Figure 6.15: Maximum damage versus time for case ISL-3 Letters correspond to the letters of the viscosity snapshots in figure 6.17. 


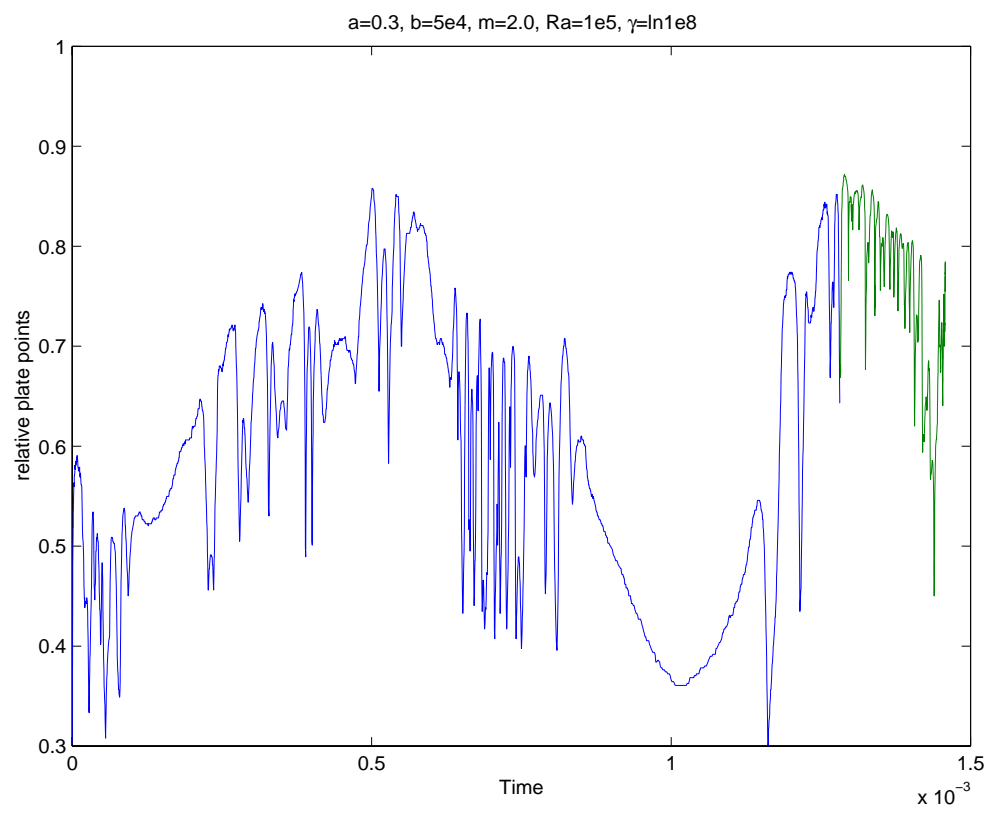

Figure 6.16: Relative number of plate points versus time for case ISL-3

(1) The lithosphere is weaker here, as expected from the simple analysis of the rheology in section 3.3. While the subduction in case ISL-1 stops shortly after the point I used as initial condition for this case, it permanently proceeds here (see figure 6.15).

(2) An already existing subduction zone suddenly changes its character here, developing the lense-shaped coat around the downwelling, which is typical for last chapter's regime IV (figures 6.17). Moreover the angle of subduction is lower than 90 degrees and the asymmetry is also visible in the evolution of the lense-shaped LVZ around the downwelling (figures $6.17 \mathrm{~g}-6.17 \mathrm{i})$. However, the fast subduction of material related to regime IV convection and the corresponding widening of the passive spreading center appear very non-plate-like. (3) Since significant passive spreading is present during the whole calculation the surface velocity is very uniform (see the surface velocity snapshots corresponding to figures $6.17 \mathrm{e}$ and $6.17 \mathrm{i}$ and the high number of relative plate-points in figure 6.16).

\subsection{Case ISL-4}

Finally, I discuss the influence of the advection term in the damage equation on the convection pattern by decreasing $a$ and $b$ by a factor of four compared to case ISL-1. The initial conditions for temperature and damage are the same as in case ISL-1. 
a
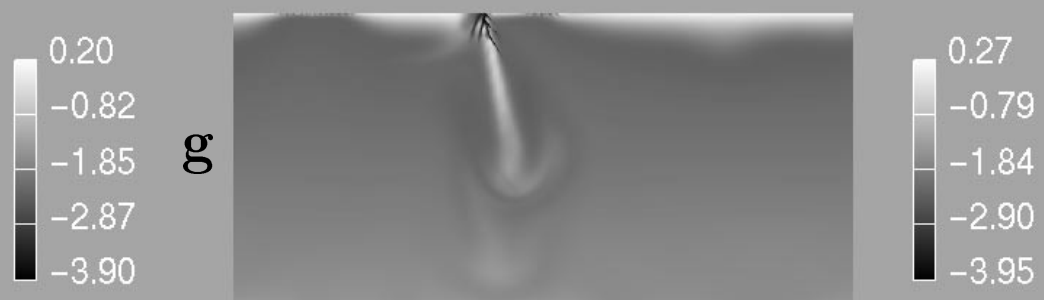

b
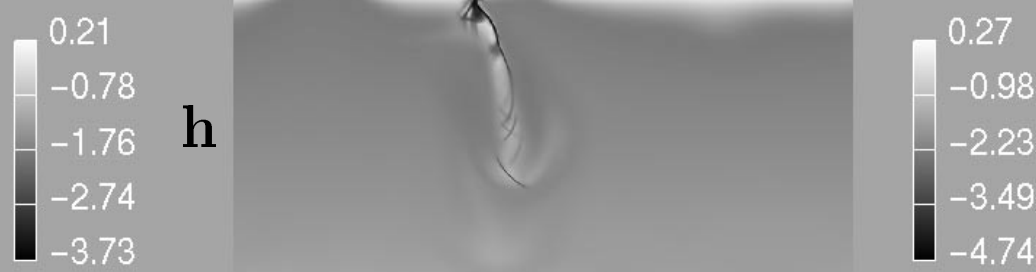

C
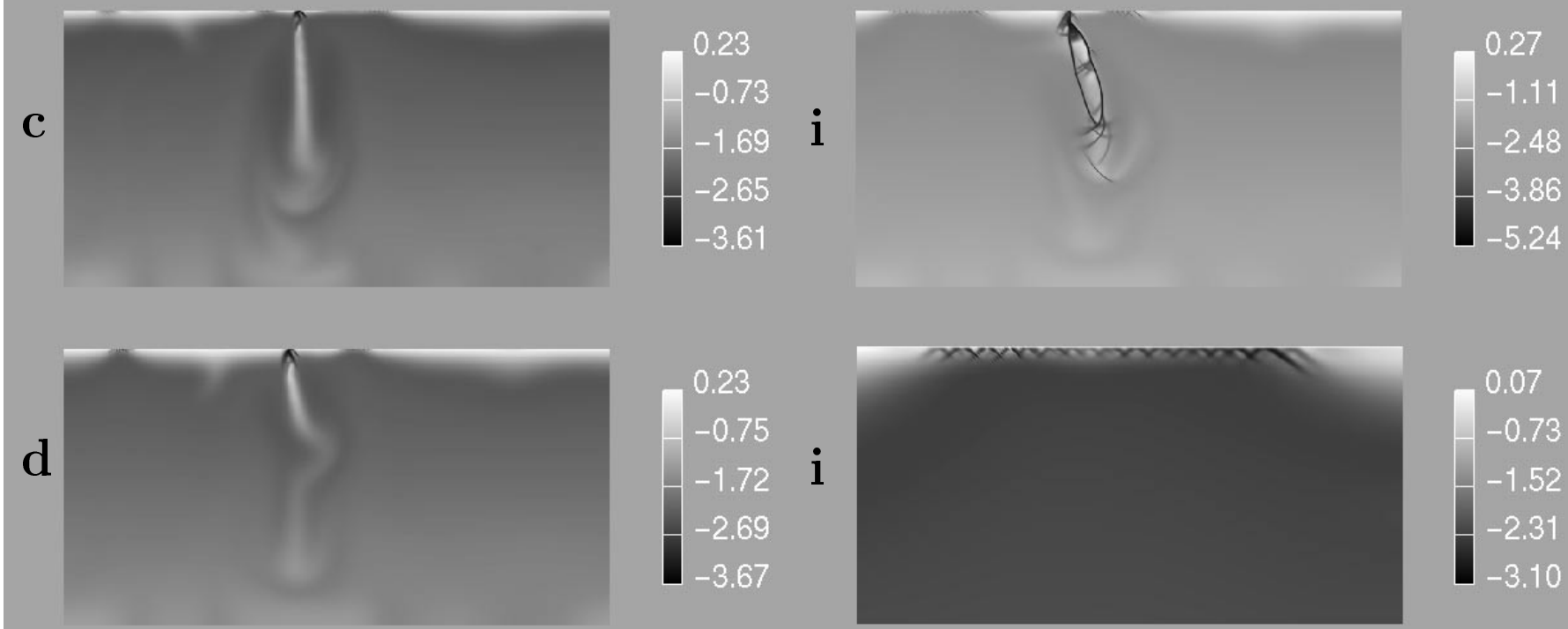

e
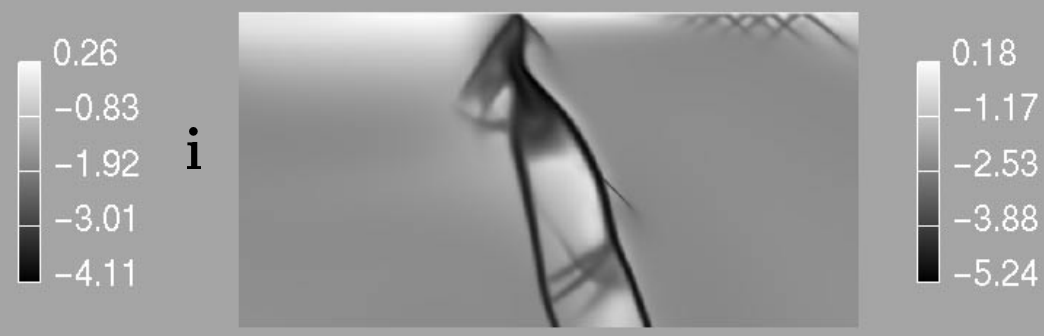

Figure 6.17: Viscosity snapshots for case ISL-3 $\left(a=0.3, b=5 \cdot 10^{4}, m=\right.$ 2.0, $\left.R a=10^{5}, \gamma=\ln 10^{8}\right)$ plus magnifications of frame " $i$ ". 

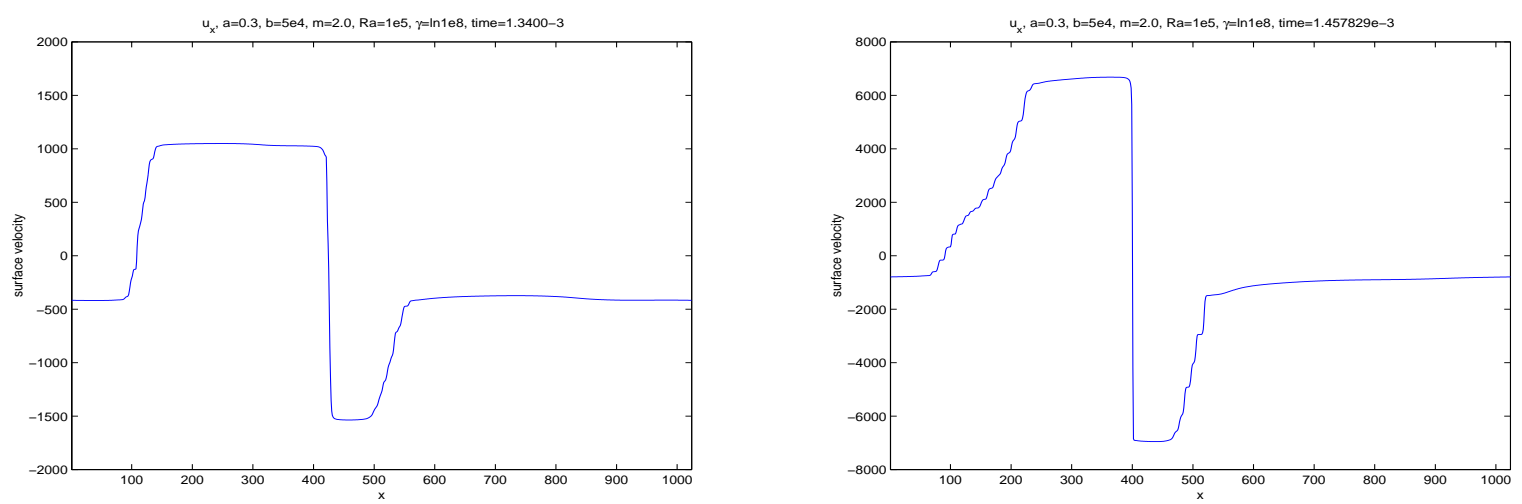

Figure 6.18: Surface velocities for case ISL-3 $\left(a=0.3, b=5 \cdot 10^{4}, m=2.0, R a=10^{5}, \gamma=\right.$ $\left.\ln 10^{8}\right)$, corresponding to the viscosity fields "e" (left) and " $i$ " (right).

Figure 6.19 shows that compared to case ISL-1 this case is significantly less episodic. While in case ISL-1 five or six active (high damage) episodes occur before a statistical steady state is reached at times around 0.03 , case ISL-4 has only two active episodes before a statistical steady state is established at a time of 0.015 . As expected the lower values of $a$ and $b$ lead to a significant decrease of the maximum damage (figure 6.19) and more linear viscosity structures than in case ISL-1 (for example figures $6.21 \mathrm{a}, \mathrm{b}$ and i). Moreover passive spreading centers seem to defocus here (compare for example figures 6.21b and 6.7c). As a consequence, the surface velocity is usually less homogeneous in case ISL-4 than in case ISL-1 (see the number of plate points in figure 6.20 and the surface velocity snapshots in figure 6.22).

In summary the increase of the source and the sink term parameters in the damage equation

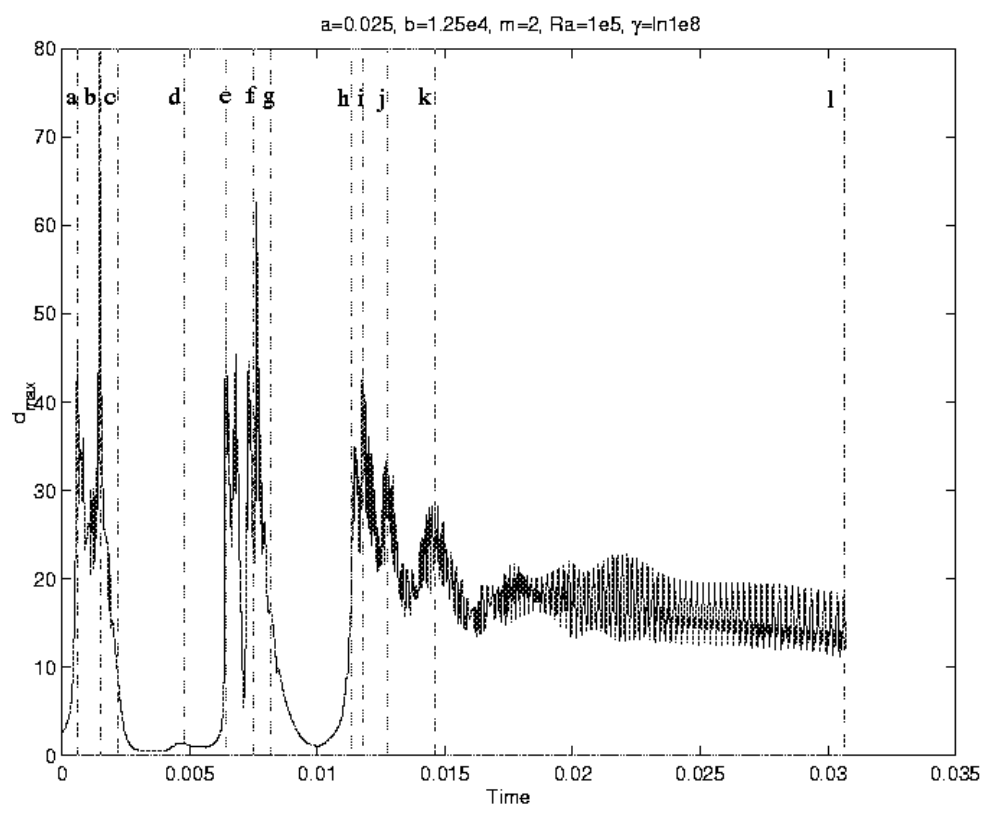

Figure 6.19: Maximum damage versus time for case ISL-4 


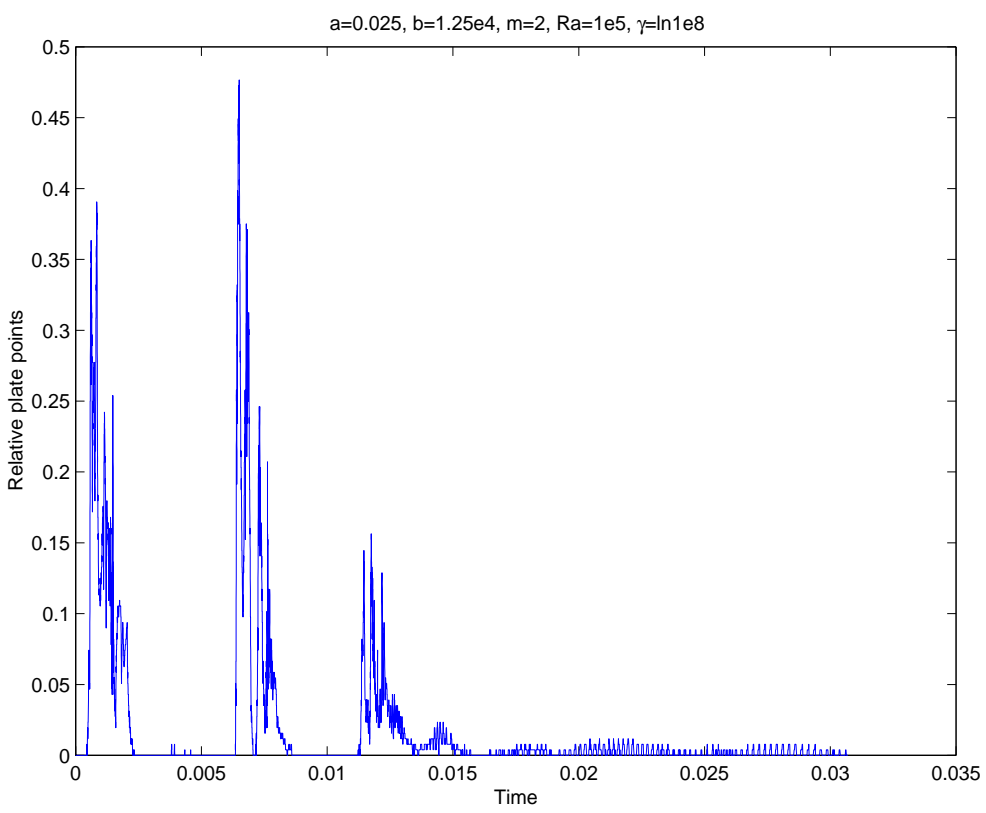

Figure 6.20: Relative number of plate points versus time for case ISL-4

( $a$ and $b$ ) encourages episodic behavior.

\subsection{Discussion and Conclusions}

With respect to the four questions to internally heated systems I addressed in the introduction of this chapter, I conclude the following:

- Localized passive spreading centers can be obtained for internally heated systems. However, the presence of passive spreading centers is not necessarily obtained even when LVZs are present above a downwelling (see case ISL-1).

- In general, asymmetry of subduction can be obtained in internally heated models. However, asymmetry in the plate velocities at a subduction zone (see ISL-1) and asymmetry in the subduction angle (see ISL-3) are not as strictly related as in bottom heated models.

- A classification of this section's results into the four regimes introduced in the previous chapter is difficult, because ...

(1) ... the results here are significantly more time dependent than the examples for bottom heated models. It therefore makes sense to separate different time-intervals where the model is in different regimes during a calculation.

2. .. in bottom heated systems the transition between the convective regimes II and IV is never observed when $a$ is increased. Here, however, this transition is evident: 
Figure 6.21: Previous page: Viscosity snapshots for case ISL-4
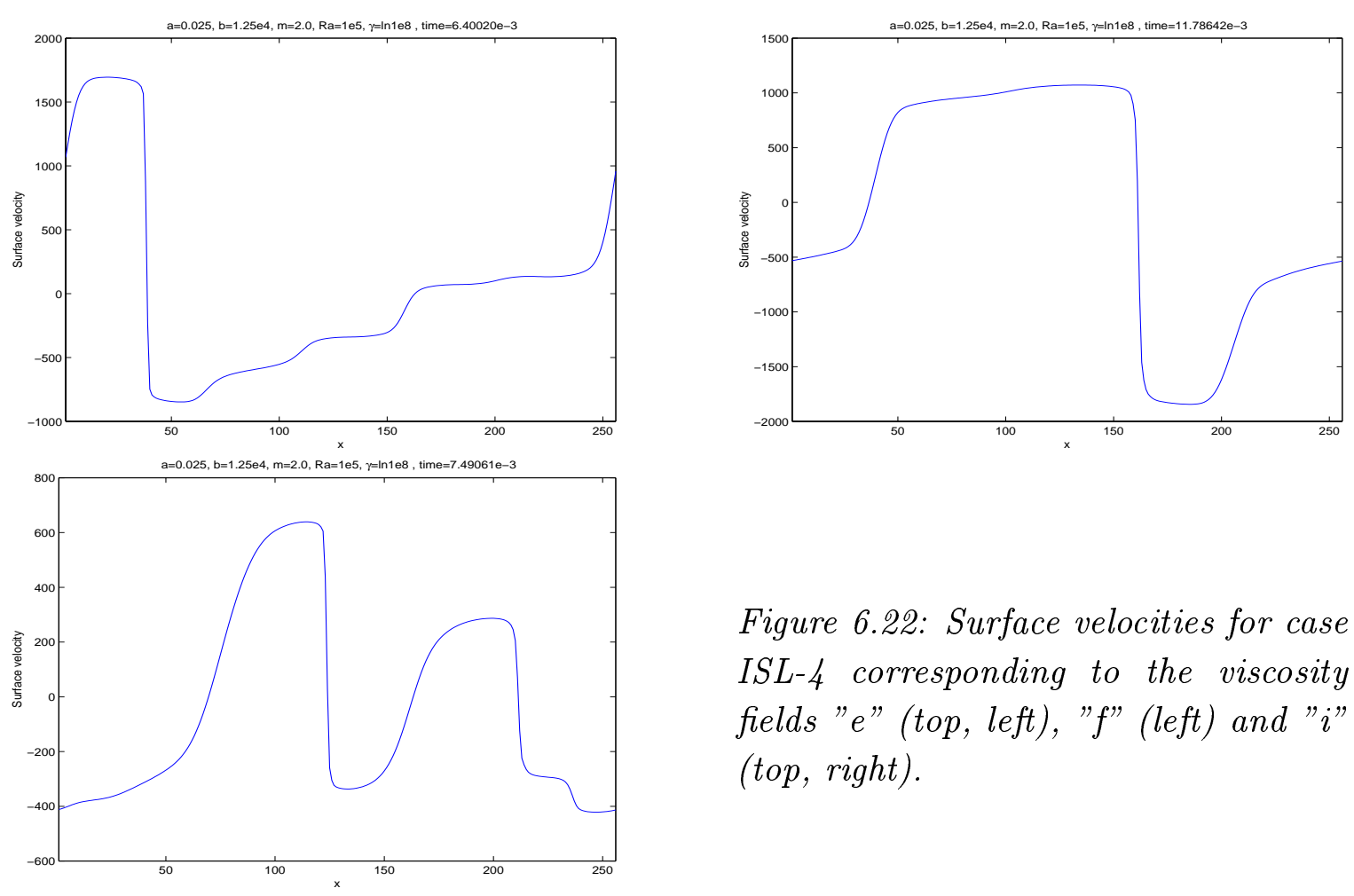

Figure 6.22: Surface velocities for case ISL-4 corresponding to the viscosity fields "e" (top, left), "f" (left) and " $i "$ (top, right).

A system without any influence of damage can very quickly develop downwellings covered by lense-shaped LVZs (see case ISL-1, fourth cycle).

Because of these problems I have to reorganize my regime definitions. Instead of four regimes I will use only the following three in the future:

Low Damage Regime (LDR): This is equivalent to the former regime I. Although the damage may not completely vanish in the box it is too small to significantly influence the structure of convection for at least two mantle overturn time.

Variable Damage Regime (VDR): It is a synthesis of the former regimes II and IV. Characteristics of this regime are strong temporal variations of the damage usually linked to significant changes in the intensity of downwellings (amount / velocity of subducting material). Since different mechanisms can be responsible for the damage variations I distinguish three different types for this regime:

Type II: Damage changes from very small to intermediate values. Zones of intermediate damage at different positions (passive spreading centers, above or below a downwelling) are not connected. This basically corresponds to the old regime II.

Type IV: Damage changes from intermediate to high values. LVZs at different 
positions (see above) become temporary connected. The name comes from the old regime IV.

Type VI: This is a combination of types II and IV: Damage increases quickly from low to very high values, LVZs quickly develop at different positions and become connected to each other.

To distinguish this regime from a simple transition between the Low Damage Regime and the following Homogeneous Damage Regime I define that at least two cycles of episodic behavior have to be present for this Variable Damage Regime.

Homogeneous Damage Regime (HDR): This is the old regime III. Damage has intermediate values and significantly influences the viscosity for at least two mantle overturn times at the same position. The variations in the damage and the structures of the downwellings are only weakly time-dependent.

Since a calculation can run through several of these regimes transition intervals between them are naturally obtained.

Subregimes, like in the previous chapter (IIIa for example) are not introduced again. Except for the Low Damage Regime self-focusing or non-self-focusing LVZs are obtained in all the other regimes especially in all types of the Variable Damage Regime (see case ISL-1 for focused type II LVZs in the VDR and case IST-1 (below) for non-focused Type VI LVZs).

- A simple $a$ dependence of the different regimes as for bottom heated cases can not be obtained. However, increasing $a$ still favors the HDR and the VDR type IV and type VI instead of the LDR and the VDR type II. In general episodic behavior seems to be much more common in internally heated systems than in bottom heated systems. 


\section{Chapter 7}

\section{Internally heated models II}

According to Solomatov (1995) convection on Earth would occur in the stagnant lid regime if viscosity would be only temperature-dependent. Therefore, internally heated models, which would produce a stagnant lid without damage influence are investigated now.

\subsection{Introduction}

Motivates the investigation of stagnant lid convection in the context of these thesis.

\subsection{Breaking of the stagnant lid}

The minimum $a$ values needed to break a stagnant lid are determined $(R a, \gamma$ variable;

$b, m$ fixed). A simple explaintion for $a_{b r e a k}$ is given and its value for Earth is estimated.

\subsection{Temporal evolution of a broken lid}

The temporal evolution of a broken lid is considered for one example.

Variations of $a$ and $a, b$ are presented.

\subsection{Additional depth-dependence of viscosity}

Different kinds of additional depth dependences of viscosity are tested. 


\subsection{Introduction}

In the previous chapter I showed that in internally heated simulations good plate-like behavior in terms of focused LVZs, uniform surface velocities and asymmetrical subduction is in principal possible. However, two problems remain:

(1) Especially the uniformity of the surface velocities strongly depends on the ability of the model to produce passive spreading centers. But these are not necessarily obtained even if well developed LVZs are present above downwellings.

(2) Solomatov (1995) shows that convection on Earth would occur in the Stagnant Lid Regime, if viscosity would be only temperature dependent. However, the values for $R a$ and $\gamma$ used in the previous simulations are close to the boundary between the low viscosity and the sluggish lid convective regime for purely temperature-dependent rheology.

Models with $R a$ and $\gamma$ values which would produce stagnant lid convection for undamaged material can be expected to address both problems:

(1) Since the differences between localized downwellings and diffuse upwellings are smaller for stagnant lid convection a more realistic evolution of passive spreading centers might be obtained.

(2) Following Solomatov (1995) these models are obviously more Earth-like.

\subsection{Breaking of the stagnant lid}

\subsubsection{Method}

In this section I investigate which values for $a$ are at least necessary to break the stagnant lids obtained for different values of $R a$ and $\gamma$. These values $a_{\text {break }}(R a, \gamma)$ are determined for fixed $b=5 \cdot 10^{4}$ (sink of damage) and $m=1.5$ (damage-dependence of viscosity). For each pair of $R a$ and $\gamma$, several calculations are performed using the (statistical) steady-state temperature field of the corresponding simulation without damage and a damage parameter of unity everywhere as initial conditions. $a$ is increased by a factor of two between the calculations until a breaking process is visible in a calculation ${ }^{1}$. An overview over the $R a-\gamma$ pairs investigated here is given in table 7.1 .

\subsubsection{Results}

A first qualitative result of these simulations is that always both - a significant downwelling and a passive spreading center - develop at the breaking of the stagnant lid.

The reason for this important difference to simulations initiated with sluggish lid convection

\footnotetext{
${ }^{1}$ The approximate value of $a_{b r e a k}$ can be quickly determined by calculating the source $a \sigma \dot{\varepsilon}$ and sink $b d e^{\gamma T}$ term for the initial conditions. Since advection is minor in the lid it can only break, if source and sink term are approximately equal. However, all of the calculations presented here ran until the breaking was visible in the viscosity field.
} 


\begin{tabular}{|rc|cc|rrrr|}
\hline Series & Case & $R a$ & $\gamma$ & $\langle\Delta \eta\rangle$ & $\left\langle\Delta u_{\text {surf }}\right\rangle$ & $D(\cdot 128)$ & $a_{\text {break }}$ \\
\hline \hline BR4 & 1 & $10^{4}$ & $\ln 10^{26}$ & $7.17 \cdot 10^{3}$ & 29.0 & 19 & 12.00 \\
BR4 & 2 & $10^{4}$ & $\ln 10^{29}$ & $1.23 \cdot 10^{4}$ & 20.0 & 17 & 24.00 \\
BR4 & 3 & $10^{4}$ & $\ln 10^{38}$ & $5.02 \cdot 10^{4}$ & 8.0 & 14 & 96.00 \\
BR4 & 4 & $10^{4}$ & $\ln 10^{62}$ & $3.39 \cdot 10^{5}$ & 3.5 & 11 & 384.00 \\
\hline BR5 & 1 & $10^{5}$ & $\ln 10^{50}$ & $1.36 \cdot 10^{4}$ & 70.0 & 10 & 0.75 \\
BR5 & 2 & $10^{5}$ & $\ln 10^{62}$ & $3.57 \cdot 10^{4}$ & 25.0 & 9 & 6.00 \\
BR5 & 3 & $10^{5}$ & $\ln 10^{74}$ & $7.30 \cdot 10^{4}$ & 15.0 & 8 & 12.00 \\
\hline
\end{tabular}

Table 7.1: Overview over the parameter studies about the breaking of a stagnant lid. Some values $(<>)$ are averaged over a characteristic timeintervall.

(see the previous chapter) is the smaller difference in the shape of up- and downwellings: The sluggish lid regime in internally heated models is characterized by well developed localized downwellings and broad, diffuse upwellings. The strain rate is therefore much higher above the downwelling than anywhere else at the surface and thus LVZs above the downwelling are much easier obtained than passive spreading centers.

In the Stagnant Lid Regime the parameter $\gamma$ is so large that an immobile lid which hardly participates in the convective process evolves at the top of the box. The few weak downwellings present in this regime, do not feed on material from the surface of the box, but only on material from the bottom of the thermal boundary layer.

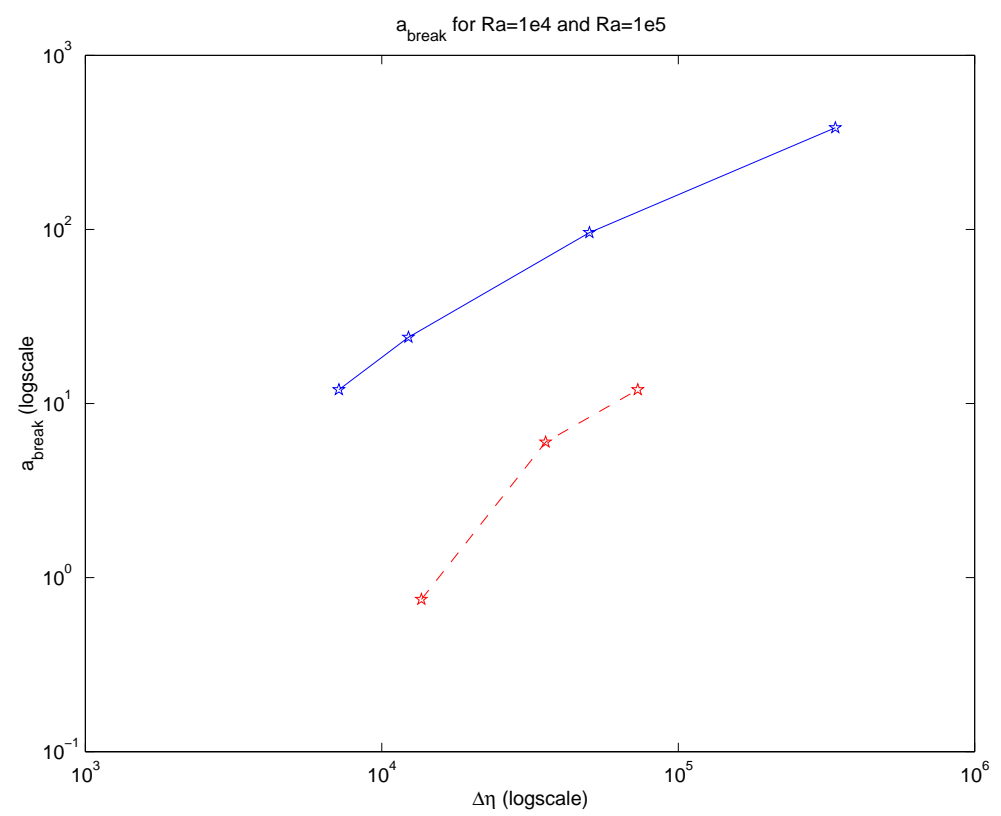

Figure 7.1: Size of the damage source (a) necessary to break the stagnant lid of a model with the same dimensionless numbers $(R a, \gamma)$ but only temperature-dependent viscosity. The solid curve uses $R a=10^{4}$, the dashed $R a=10^{5} . b=5 \cdot 10^{4}$ and $m=1.5$ are assumed. 

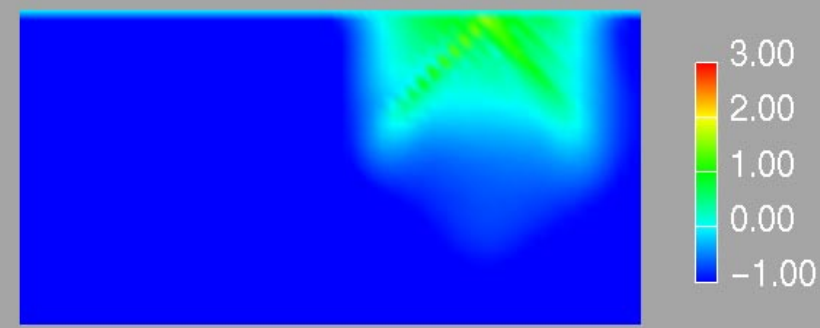

C
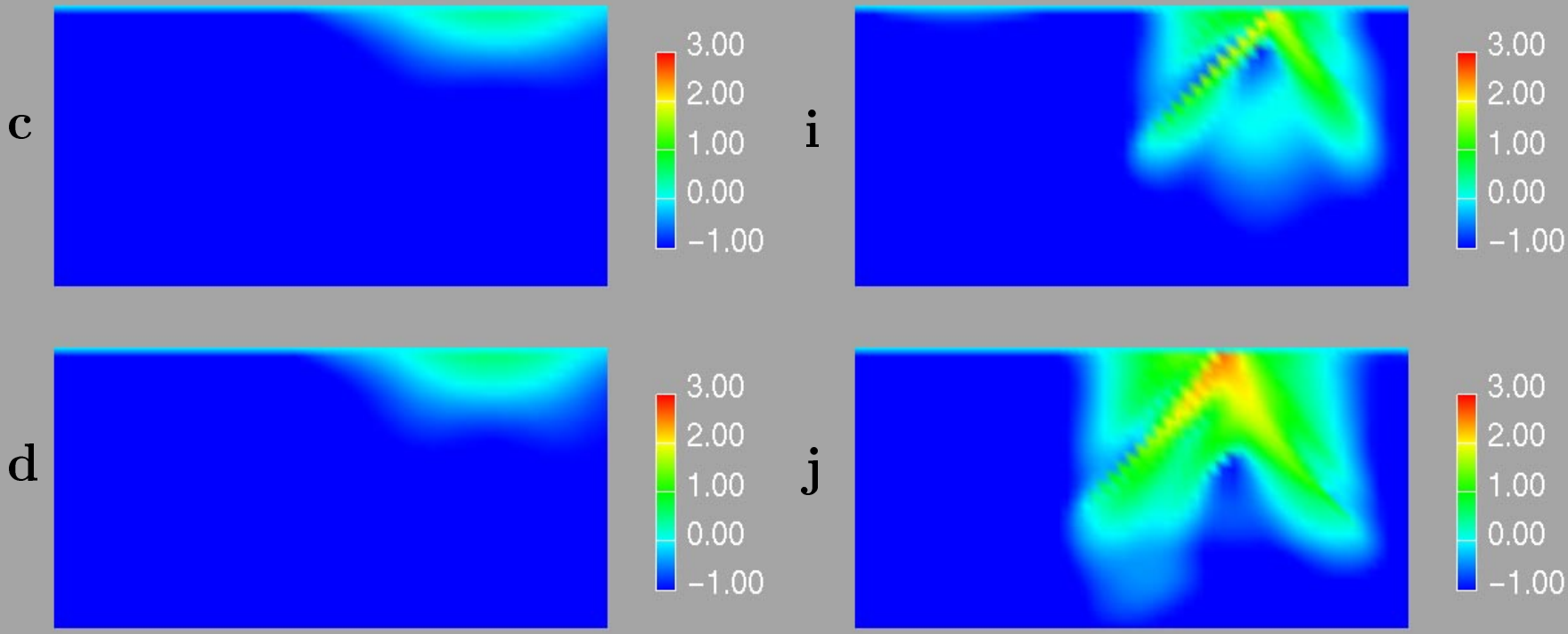

e
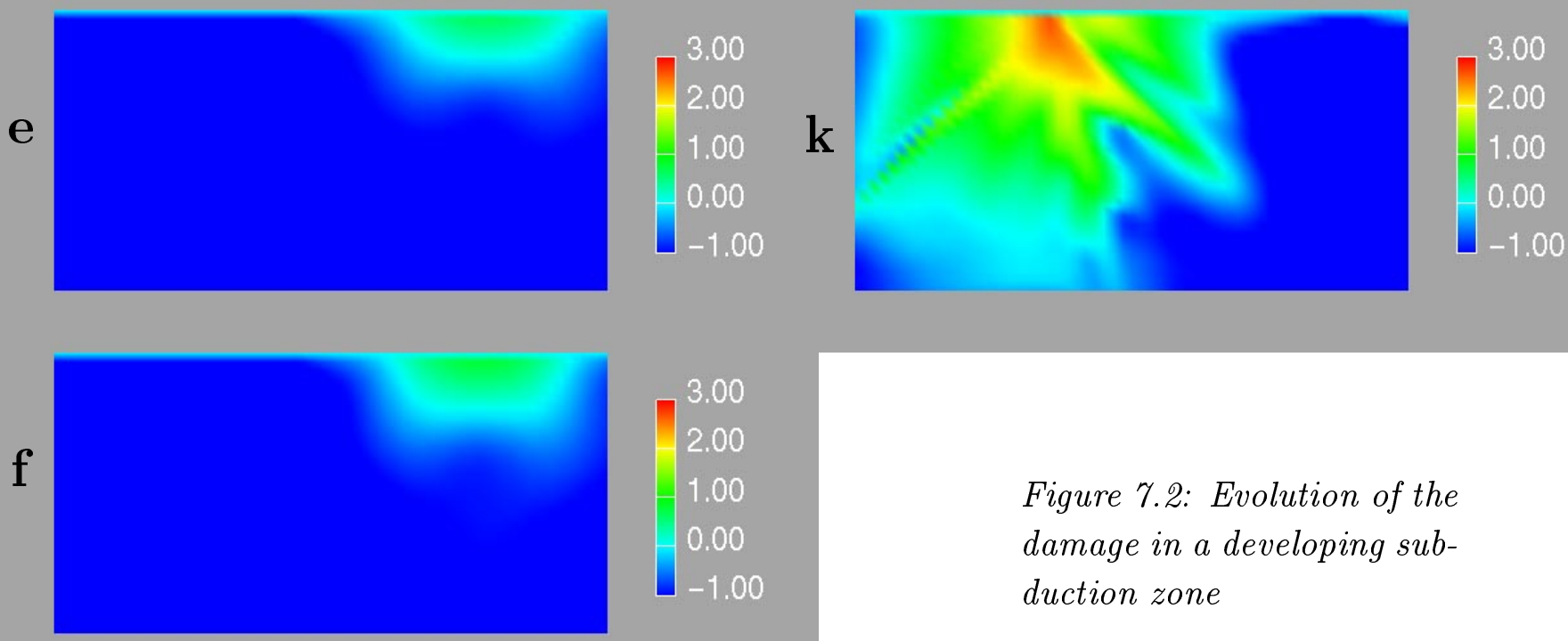

Figure 7.2: Evolution of the damage in a developing subduction zone 
Therefore, the strain rate at the surface of the box is comparatively uniform and the simultaneous development of subduction zones and passive spreading centers in models with damage dependence is possible.

The second result are the $a_{b r e a k}(R a, \Delta \eta)$ curves $^{2}$ shown in figure 7.1. Qualitatively, $a_{b r e a k}$ increases with increasing viscosity contrast and decreasing Rayleigh number.

In the following I will derive an empiric relation for $a_{\text {break }}$ that is based on general physical considerations:

(1) The temporal evolution of the damage in a breaking lid (figure 7.2) shows that the break starts at the top of the lithosphere and slowly propagates inwards. Because of its strong dependence on temperature the intermediate lithosphere significantly inhibits the propagation of damage and the breaking of the stagnant lid.

(2) Therefore surface quantities are important to initiate the breaking process. The only laterally variable surface quantity is the surface velocity $u_{\text {surf }}$.

(3) Let $\Delta u_{\text {surf }}\left(\hat{\left.\Delta u_{\text {surf }}\right)}\right)$ be the maximum difference in the surface velocity $\left(u_{\text {surf,max }}-\right.$ $\left.u_{\text {surf,min }}\right)$ for fixed $R a$ and $\Delta \eta(\hat{\Delta \eta})$, with $\hat{\Delta u_{\text {surf }}}=c \cdot \Delta u_{\text {surf }}, c>1$. Since convection occurs basically in two cells in all simulations also $\hat{\dot{\varepsilon}}_{\text {surf }}=c \cdot \dot{\varepsilon}_{\text {surf }}$ holds. For $\eta_{T}=1$ and $d \equiv 1$ at the surface the advection term can be neglected and the damage equation reduces to

$$
\begin{aligned}
\frac{\partial d}{\partial t} & =a \dot{\varepsilon}_{\text {surf }}^{2}-b \\
& =\frac{a}{c^{2}} \hat{\dot{\varepsilon}}_{\text {surf }}^{2}-b \\
& =\hat{a} \hat{\dot{\varepsilon}}_{\text {surf }}^{2}-\hat{b}
\end{aligned}
$$

with $\hat{b}=b$ and $\hat{a}=a / c^{2}$. I finally obtain

$$
a_{\text {break }} \sim \Delta u_{\text {surf }}^{-2}
$$

(4) Figure 7.3 shows the $a_{\text {break }}--\Delta u_{\text {surf }}$ relation as derived from the numerical simulations together with relation 7.4. It demonstrates two problems: (a) Equation 7.4 is not the best fit for the experimental $a_{\text {break }}\left(\Delta u_{\text {surf }}\right)$ curve. (b) For different Rayleigh numbers different $a_{\text {break }}$ values are obtained even for the same $\Delta u_{\text {surf }}$ values.

(5) Additional quantities have to be considered in the model.

(6) Idea:

$$
a_{\text {break }} \sim D^{n}
$$

where $D$ is the thickness of the (highly viscous) thermal boundary layer and $n$ is an experimentally determined exponent.

The motivation for this idea is the following: Damage of the stagnant lid starts at its surface

\footnotetext{
${ }^{2}$ Instead of $\gamma$ the use of the real viscosity contrast $\Delta \eta$ is more sensible in this context, since $\Delta \eta$ is the quantity which immediately influences the convective structure. Using $\gamma$ would be ambiguous since it implies different $\Delta \eta$ 's due to the inconsistent temperature in the box.
} 


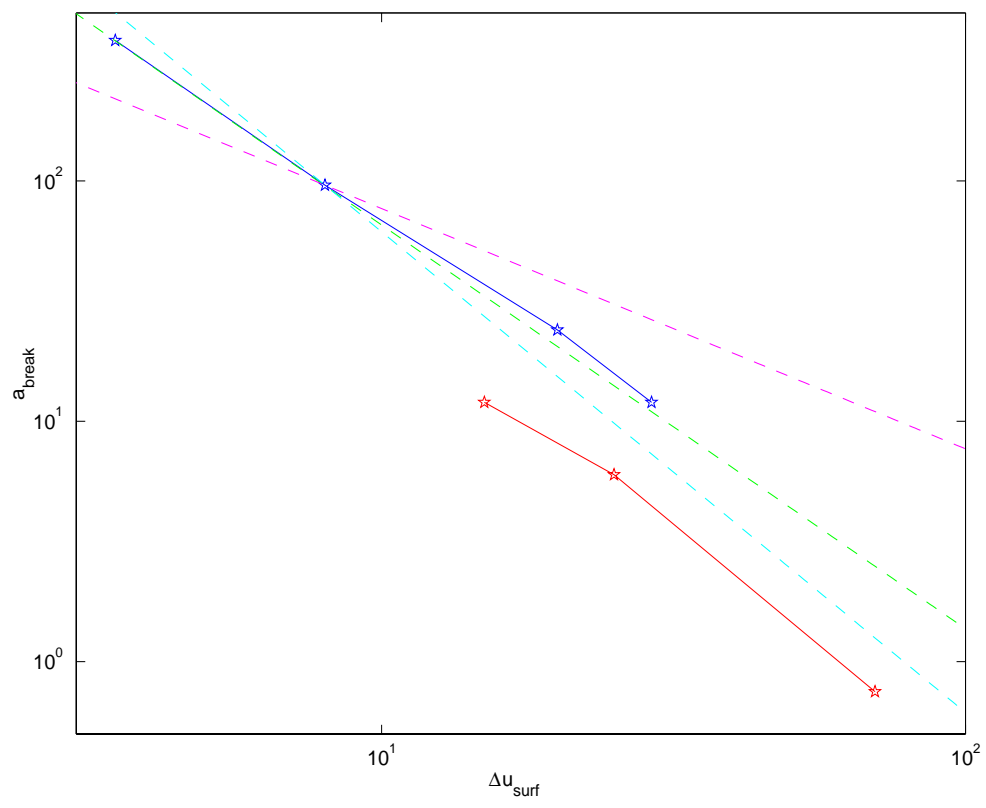

Figure 7.3: $a_{\text {break }}$ versus $\Delta u_{\text {surf }}$ for $R a=10^{4}$ (blue curve) and $R a=10^{5}$ (red curve). Also included are curves $a_{\text {break }} \sim \Delta u_{\text {surf }}^{-1}, a_{\text {break }} \sim \Delta u_{\text {surf }}^{-1.68}$ and $a_{\text {break }} \sim \Delta u_{\text {surf }}^{-2}$ (dashed lines).

and propagates inwards although the healing of the damage is initially much faster there than its production (see figure 7.4). How far the damage can propagate depends on its initial value at the surface and therefore on $a$. If the propagation depth is not deeper than the

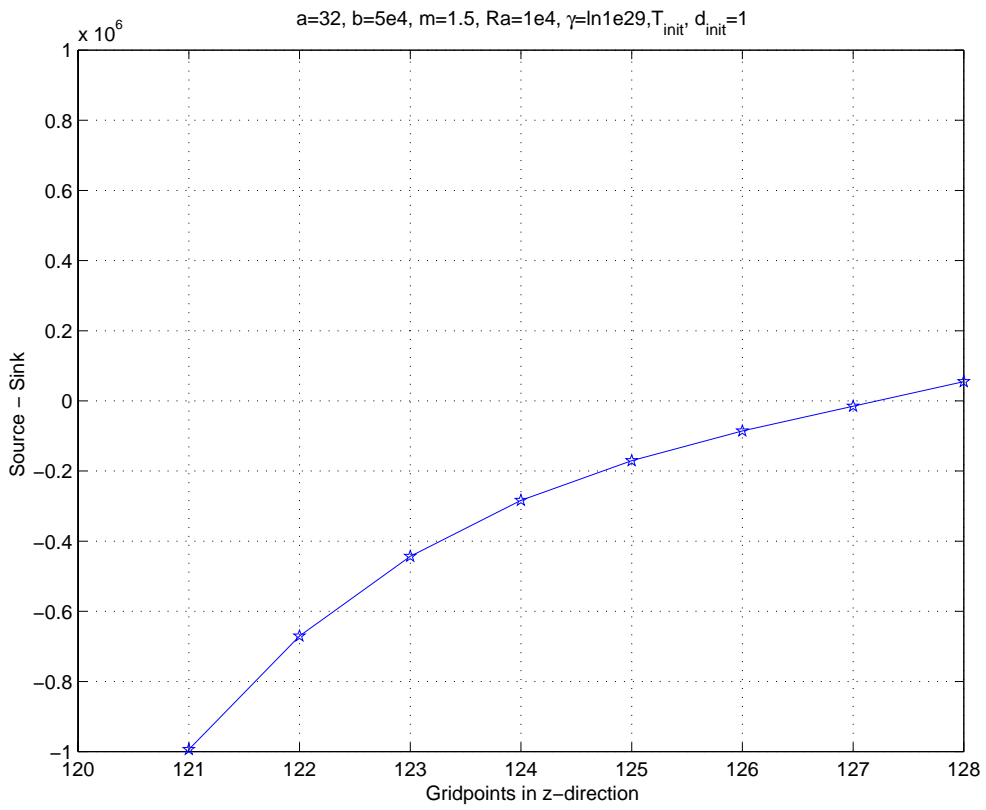

Figure 7.4: Depth profile of the difference between damage source and sink term for the initial conditions of case BR4-2. The box surface corresponds to grid-point 128 in z-direction. 


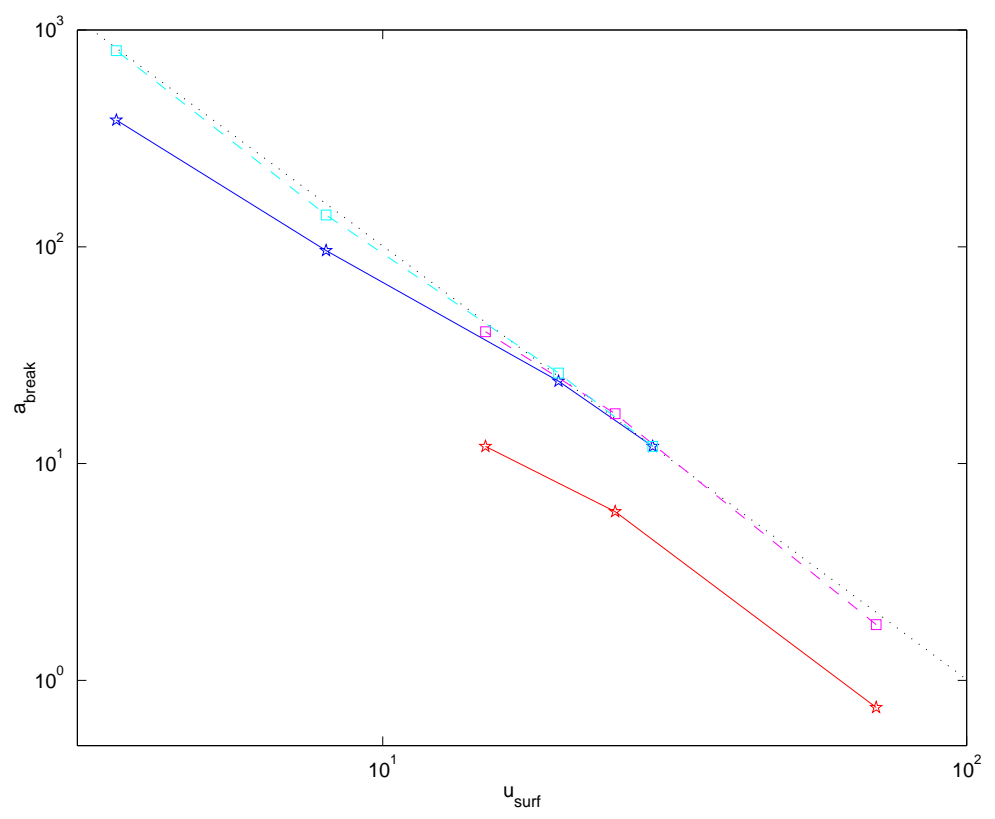

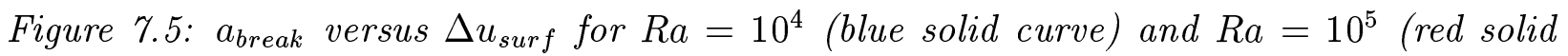
curve). Also included are the curve $a_{\text {break }}=10092 \cdot \Delta u_{\text {surf }}^{-2}$ (black dotted line) and the $a_{\text {break }}\left(\Delta u_{\text {surf }}\right)$ curves corrected with the thickness $D$ of the boundary layer (cyan dashed curve for $R a=10^{4}$ and magenta dashed curve for $R a=10^{5}$ ).

thermal boundary layer thickness the lid does not break. $a_{b r e a k}$ therefore depends on the thickness of the thermal boundary layer.

(7) Figure 7.5 shows that the best fit to experimentally derived $a_{\text {break }}--\Delta u_{\text {surf }}$ points is obtained for $n=3 / 2$ in equation 7.5. When corrected with their thermal boundary layer thickness relative to case BR4-2 $a_{\text {break }}$ can be calculated for all models according to $a_{\text {break }}=10092 \cdot u_{\text {surf }}^{-2}$.

(8) I finally obtain the following dependence:

$$
a_{\text {break }} \sim \frac{D^{3 / 2}}{\Delta u_{\text {surf }}^{2}}
$$

\subsubsection{Comparison with the Earth}

In this subsection I discuss, which value of $a_{\text {break }}$ might be expected for the Earth, assuming the mechanism I suggested here is indeed the one responsible for plate tectonics.

Using typical Earth-like parameters (see appendix) $R a \approx 10^{5}$ and $\Delta \eta \approx 10^{6}$ can be assumed for the $\mathrm{Earth}^{3}$. From figure 7.1 I extrapolate

$$
a_{\text {break,Earth }, 1} \approx 100
$$

\footnotetext{
${ }^{3}$ Note that (1) the Rayleigh number is scaled with the viscosity at the surface $(\mathrm{T}=0)$ and for a damage of one and that (2) the uppermost brittle lithosphere is not included into my models, which decreases the maximum viscosity contrast
} 
as a first approximation.

However, $a_{b r e a k, E a r t h, 1}$ is valid only for a fixed $b_{b r e a k, E a r t h, 1}=5 \cdot 10^{4}$. $a$ and $b$ can simultaneously be increased or decreased, changing the influence of the damage advection onto the convective structure: High values of $a$ and $b$ produce more focused LVZs than low values. By comparing the width of the LVZs in my models when the stagnant lid is broken with the width of fracture zones on Earth the determination of the "true" $a$ and $b$ becomes possible: Transform faults on Earth can have a width up to a few kilometers. Similar values are obtained at in case BR4-2. On a $1025 \times 513$ grid the resolution of the LVZs was just appropriate (five grid-points in each direction). Rescaled the width of these LVZs is therefore about $30 \mathrm{~km}$. However, for higher Rayleigh numbers or higher viscosity contrasts the width of LVZs increase compared to case BR4-2, because the velocity inside the box increases. By comparing the temporal evolution of different cases, for example BR4-2, IST-4 (see below) and BR5-1 (not presented here) I get the following approximation: Let $a_{b r e a k, c a s e 1}$ be the breaking value for one fixed case and $a_{\text {break,case } 2}$ the value for another case with different $R a$ and / or $\gamma$ but with the same $b$ (and $m$ ). The root-mean-square velocity $v_{r m s, c a s e 2}$ of the corresponding case with $d \equiv 1$ shall be higher than $v_{r m s, c a s e 1}$. To obtain approximately the same width of LVZs in case 2 than in case $1 a_{b r e a k, c a s e 2}$ and $b_{c a s e 2}$ have to be increased in the following way:

$$
b_{\text {case } 2, \text { new }} \approx \frac{v_{r m s, c a s e 2}}{v_{r m s, c a s e 1}} b_{\text {case } 1}
$$

Comparisons between BR4-2 and BR5-3 therefore suggest to increase the first approximation in equation 7.7 by a factor of around 8 :

$$
a_{\text {break }, \text { Earth }, 2} \approx 800,
$$

and

$$
b_{\text {break }, \text { Earth }, 2} \approx 4 \cdot 10^{5} .
$$

A last point to discuss is the value of $m$. It is fixed to 1.5 here for numerical reasons. Since $d$ is associated with the inverse grain size, values of two or three would be more realistic. Some tests (not presented here) demonstrate, that $m=2$ can result in a decrease of $a_{b r e a k}$ by a factor of two. Moreover an increase in $m$ further focuses the low viscosity zones. An additional decrease of $a$ and $b$ by another factor of two seems realistic for $m=2$ :

$$
a_{\text {break,Earth }, 3} \approx 200
$$

and

$$
b_{\text {break }, \text { Earth }, 3} \approx 2 \cdot 10^{5}
$$

This result is of course quite crude. Nevertheless it makes sense to compare it with the corresponding quantities for Earth:

In the non-dimensional damage equation 3.8

$$
b=\frac{h^{2}}{\kappa_{0}} \tilde{b}
$$


holds, where $\tilde{b}$ is the corresponding parameter in the original damage equation and $\frac{h^{2}}{\kappa_{0}}$ is the thermal diffusion time. $\tilde{b}$ is therefore the inverse decay time for the damage parameter. Using relation 7.13 and $b=2 \cdot 10^{5} \mathrm{I}$ get $1 / \tilde{b} \approx 1.5 \mathrm{Ma}$. Since passive fracture zones on Earth can survive tens of million years this decay time is much too short to be realistic and $b_{\text {break,Earth }, 3}$ is therefore much too large.

$\tilde{a}$ has the unit of an inverse energy per area in the dimensional damage equation:

$$
a=\frac{\eta_{0} \kappa}{d_{0} h^{2}} \tilde{a}
$$

Therefore $a=200$ roughly corresponds to $\tilde{a}=1.7 \mathrm{~m}^{2} / J$. Since $d$ is the inverse grain size of the material the physical interpretation for $\tilde{a}$ is as the inverse surface energy per area. In general, the dependence of the surface energy $E$ from the interface area $A$ between different grains (or different phases) is given by

$$
\frac{E}{V}=\beta \frac{A}{V}
$$

where $\beta$ is the surface energy per area and $V$ is a fixed volume. For the simple model of cubic grains $A / V=3 d$. The power per volume needed to decrease the grain size is therefore

$$
\begin{aligned}
\frac{\partial(E / V)}{\partial t} & =3 \beta \frac{\partial d}{\partial t} \\
& =3 \beta \cdot \tilde{a} \tilde{\sigma} \tilde{\dot{\varepsilon}} \\
& \leq \tilde{\sigma} \tilde{\dot{\varepsilon}}
\end{aligned}
$$

where advection and sink terms of the dimensional damage equation are neglected. The last line is true, because $\tilde{\sigma} \tilde{\dot{\varepsilon}}$ is the total power per volume which is available in the convective system and must therefore be higher than the power per volume used to increase the interface area. From the last equation I conclude

$$
3 \beta \tilde{a} \leq 1
$$

In other words: $100 \cdot 3 \beta \tilde{a}$ is the percentage the total power per volume of the system which is used to decrease the grain size of the material.

On Earth $\beta$ can vary between $0.1-1 \mathrm{~J} / \mathrm{m}^{2}$ for two-phase systems like silicates with melt or water and $10-1000 \mathrm{~J} / \mathrm{m}^{2}$ for extended fractures (see Bercovici et al. 2001 and references therein).

For the design of this model $\beta=1 \mathrm{~J} / \mathrm{m}^{2}$ appears to be an appropriate value. With $\tilde{a}=$ $1.7 \mathrm{~m}^{2} / \mathrm{J}$ around 500 percent of the total power per volume in Earth are needed to reduce the grain size in the lithosphere and initiate breaking, which of course is not possible. Similar to the breaking value I estimated for $b_{b r e a k, E a r t h, 3}, a_{b r e a k, E a r t h, 3}$ is about two or three orders of magnitude too high. Possible mechanisms to reduce these values like the depth-dependence of viscosity are presented later. 


\subsubsection{Summary}

I have shown in this section, that

(1) always both - LVZs above the downwellings and passive spreading centers - develop when a stagnant lid breaks.

(2) For constant $b$ and $m$ values the breaking value $a_{b r e a k}$ of the stagnant lid for different Rayleigh numbers $R a$ and viscosity contrasts $\gamma$ can be explained with differences in the surface velocity $\Delta u_{\text {surf }}$ and the thermal boundary layer thickness $D$ obtained for $d \equiv 1$ :

$$
a_{\text {break }} \sim D^{3 / 2} \Delta u_{\text {surf }}^{-2} .
$$

While the exponent for the surface velocity can be obtained from a simple model, the exponent for $D$ is determined from the best fit of my results.

(3) The extrapolation of the breaking parameters for the Earth $\left(R a=10^{5}, \Delta \eta=10^{6}\right)$ produces:

$a_{\text {break,Earth }, 3} \approx 200$ and

$b_{\text {break,Earth }, 3} \approx 2 \cdot 10^{5}$, assuming $m=2$.

A re-dimensionalization of these parameters shows that they are about two or three orders of magnitude to high to be realistic for the Earth. Additional mechanisms to reduce them are therefore necessary. As I will show later a depth-dependence of the viscosity might be appropriate for this reduction.

\subsection{Temporal evolution of a broken lid}

In this section the changes in the convective structure of a model with broken stagnant lid due to variations of $a$ or $a, b$ are investigated. $R a=10^{4}, \gamma=\ln 10^{29}$ and $m=1.5$ remain fixed. The initial fields for temperature and damage are usually taken from case IST-4 after the lid is first broken, completely subducted and newly developed. An overview over the simulation can be found in table 7.2.

\begin{tabular}{l|c|c|rr|c} 
Series & Case & aspect ratio & $a$ & $b$ & Regime \\
\hline \multirow{4}{*}{ IST } & 1 & 2 & 0.5 & 781.25 & VDR II - HDR \\
& 2 & 2 & 2.0 & 781.25 & VDR II - HDR \\
& 3 & 2 & 8.0 & 781.25 & VDR VI - VDR IV \\
& 4 & 2 & 8.0 & 12500.00 & VDR IV \\
& 5 & 2 & 32.0 & 12500.00 & VDR IV
\end{tabular}

Table 7.2: Overview over the parameter studies in the section 7.3. 


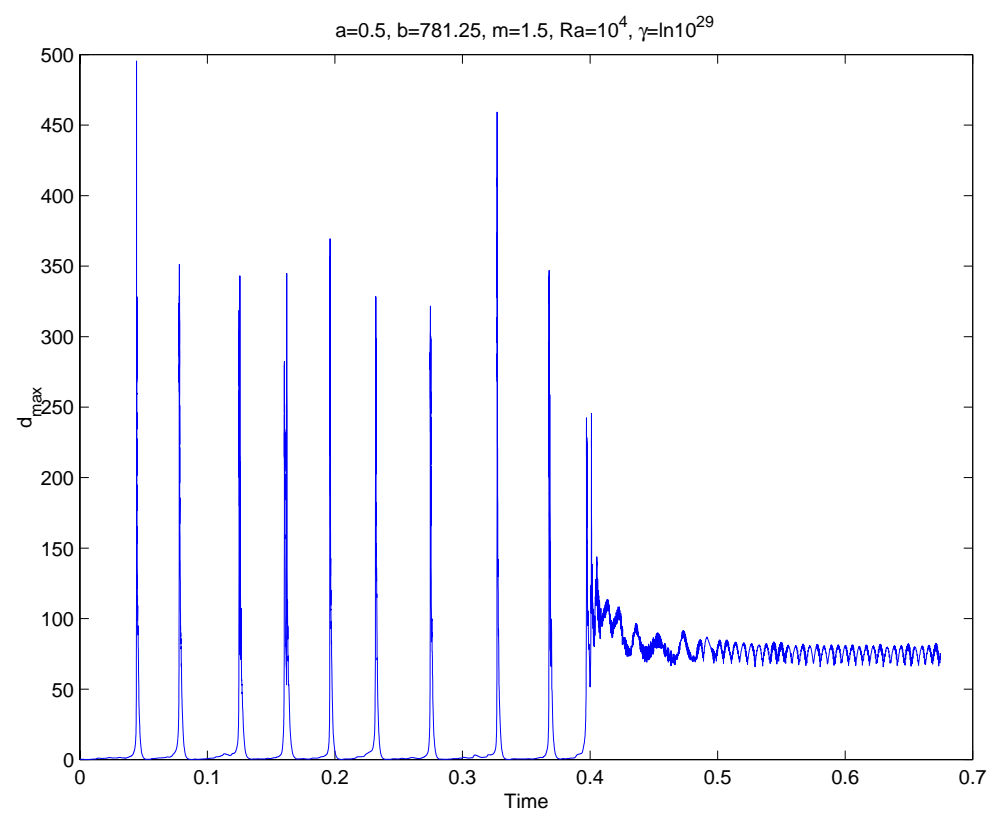

Figure 7.6: Maximum damage versus time for case IST-1

\subsubsection{Cases IST-1 - IST-3}

Figure 7.6 demonstrates that the convection is highly episodic at the beginning of the calculation. This is caused by the repeated subduction of all cold surface material into the mantle (see figure 7.3.2 as an example). However after the tenth episodic event the system reaches

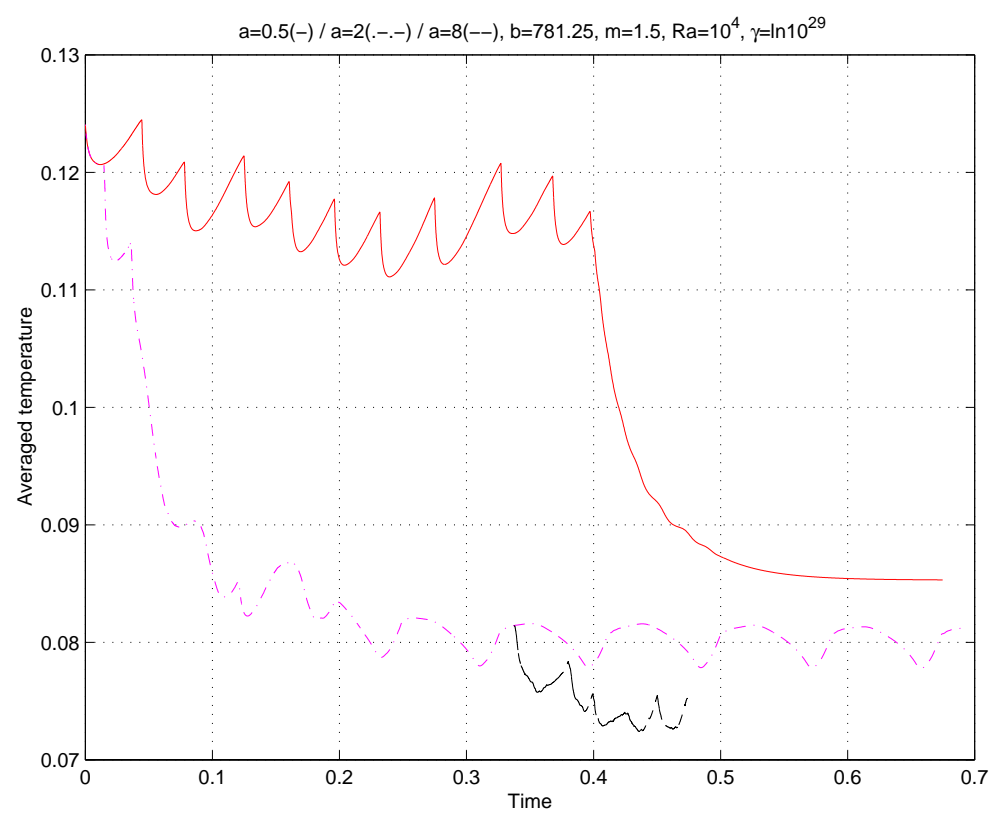

Figure 7.7: Averaged temperature in the computational domain versus time for cases IST-1 (solid line), IST-2 (dashed + dotted line) and IST-3 (dashed line). 

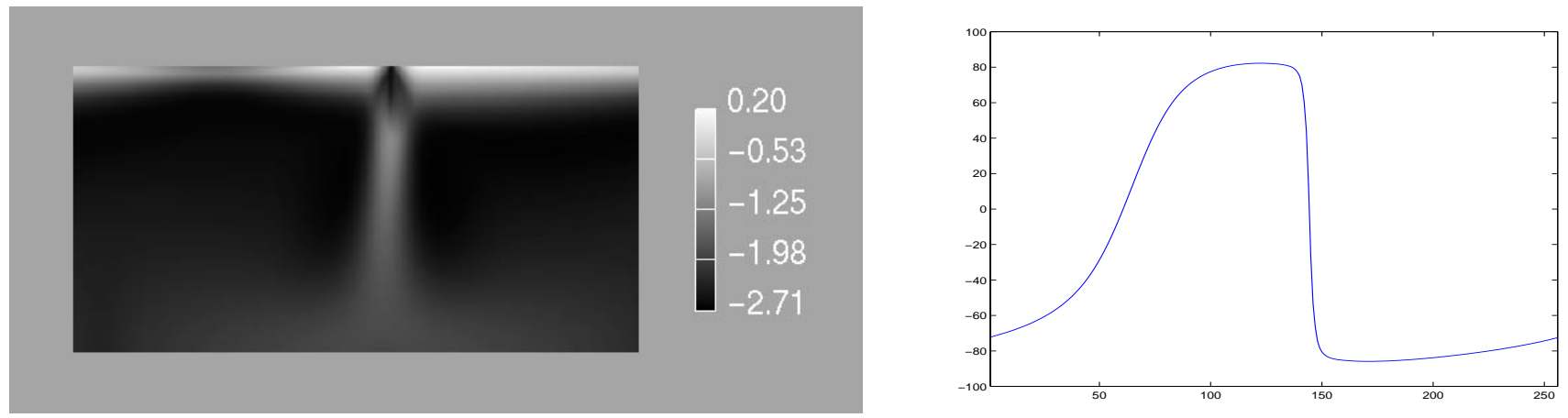

Figure 7.8: Steady state viscosity and surface velocity for case IST-1

a statistical steady state (figure 7.8). Although the passive spreading center is quite broad and the corresponding change of the surface velocity quite diffuse, it is remarkable that a permanent asymmetry is obtained in the convection pattern. The plates have different sizes and the downwelling is not entirely vertical.

Case IST-2 is started with the same initial conditions as IST-1. Although it appears less

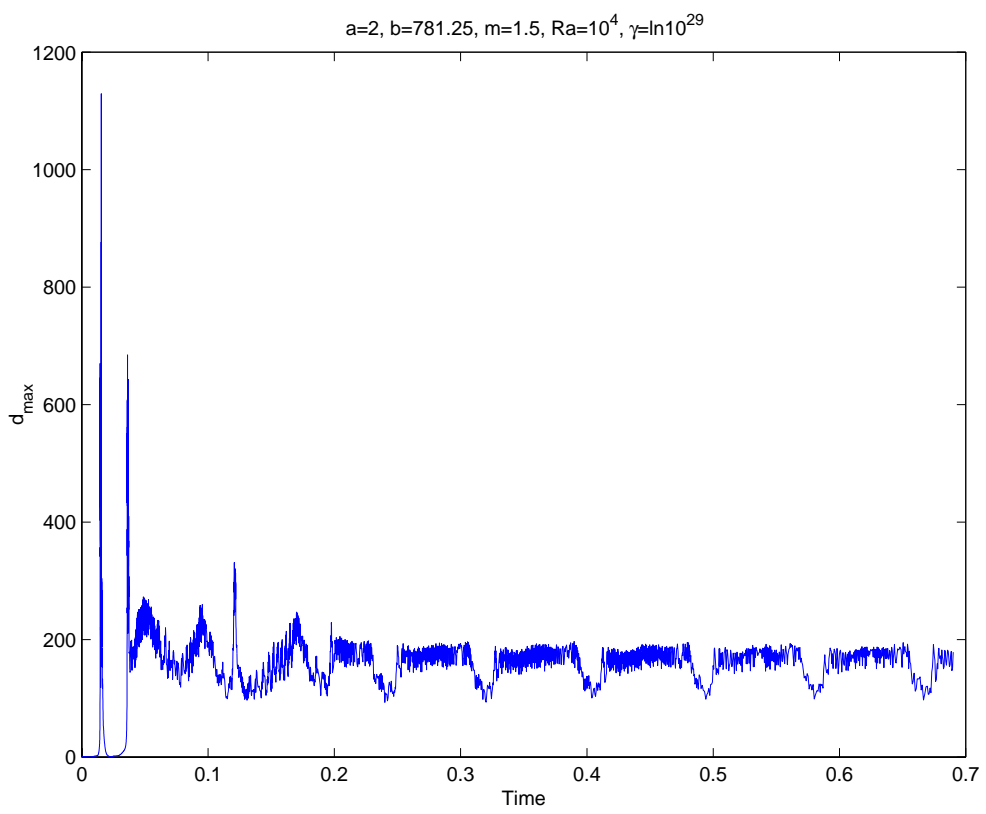

Figure 7.9: Maximum damage versus time for case IST-2

time-dependent than case IST-1 (figure 7.9) at the beginning of the calculation a stable episodic convection pattern is finally developed. For long periods during each episodic cycle one short (about $1 / 10^{\text {th }}$ box-size) and one large (about $7 / 10^{\text {th }}$ box-size) plate is present (see figure 7.10). 
a

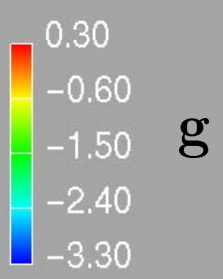

0.30
-0.60
-1.50
-2.40
-3.30
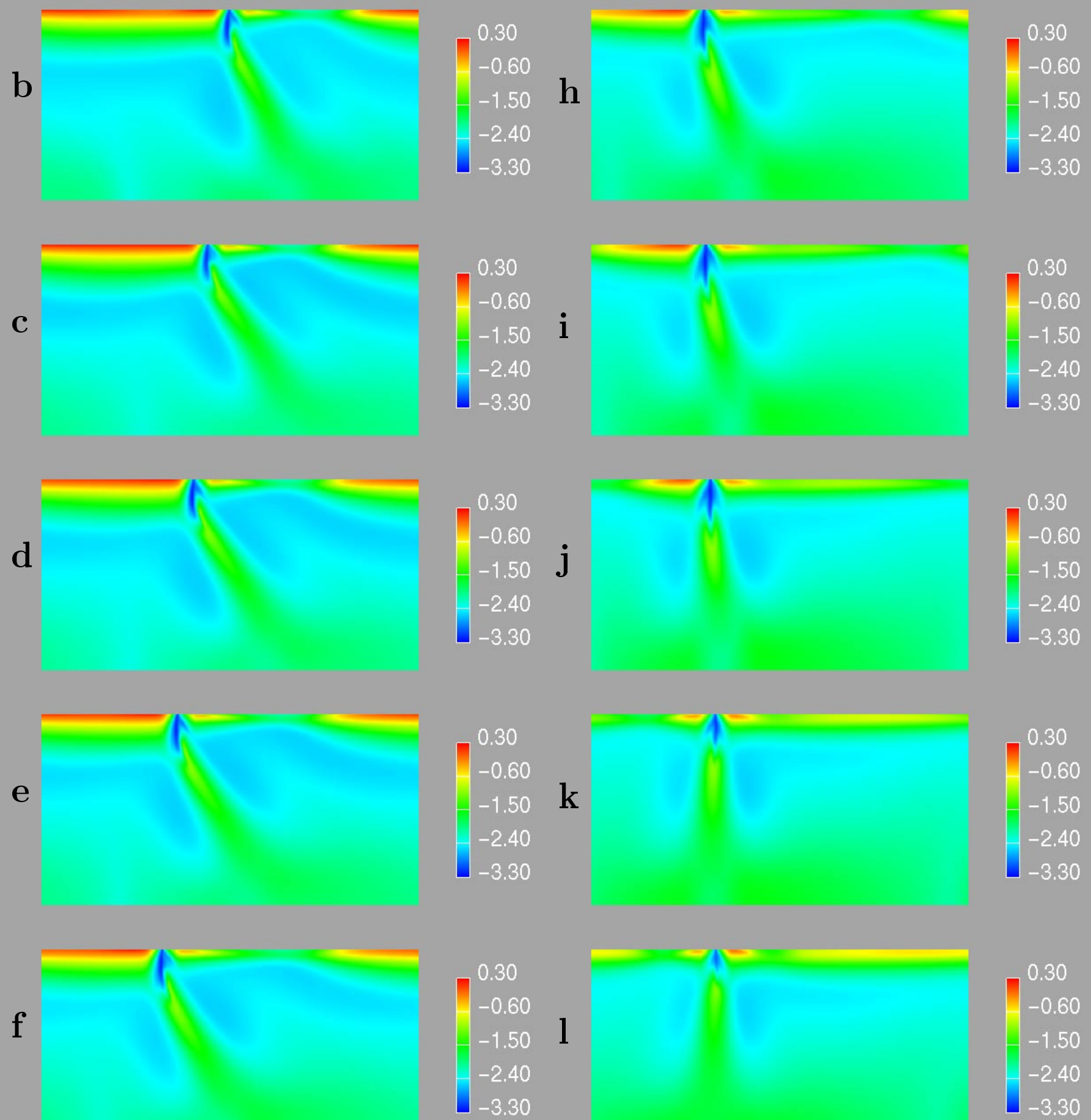
$\mathrm{m}$

$\begin{array}{ll}0.30 & \\ -0.60 & \\ -1.50 & \mathbf{S} \\ -2.40 & \\ -3.30 & \end{array}$
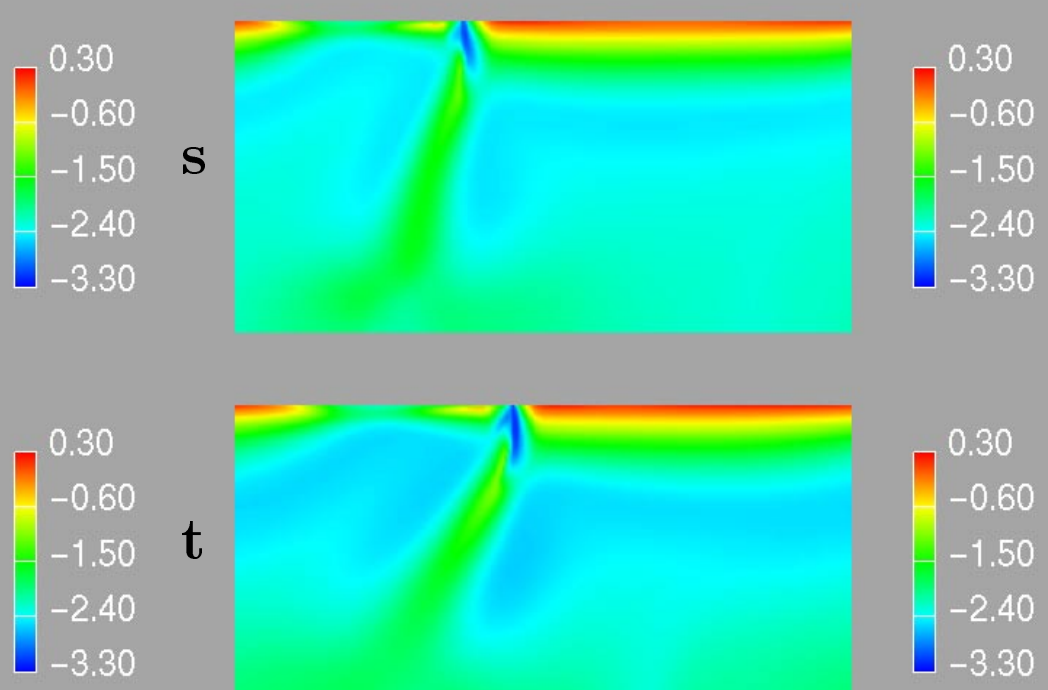

O
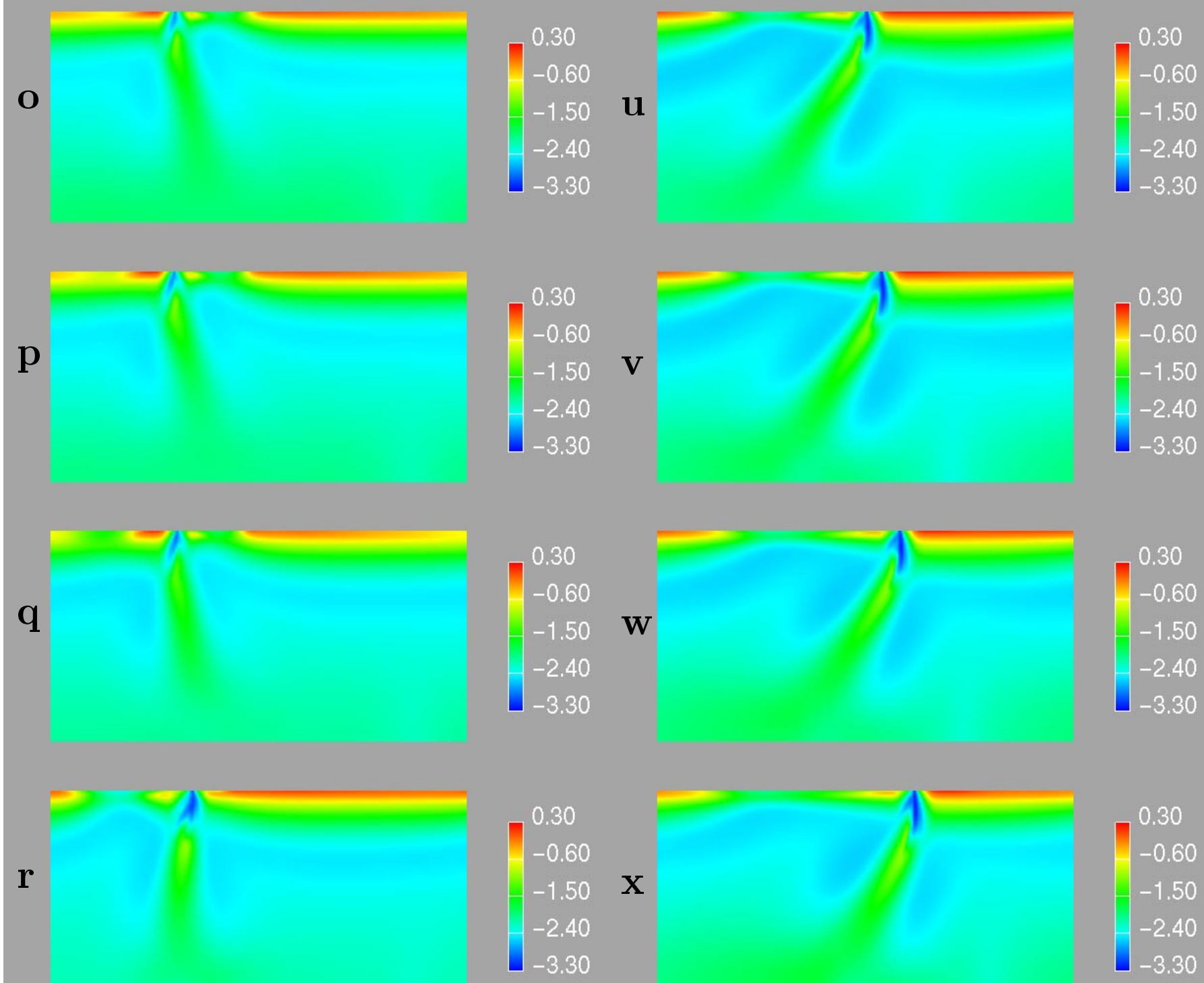
Figure 7.10: Previous page: Viscosity snapshots for case IST-2 between times 0.5 and 0.64 in non-equidistant time-steps.

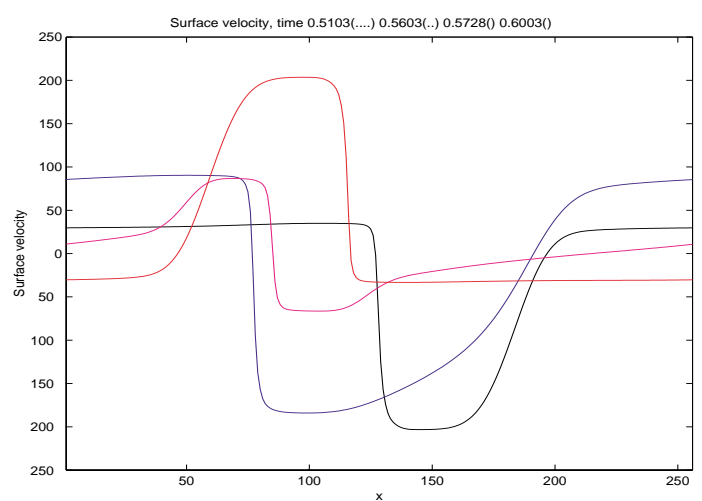

Figure 7.11: Surface velocities for case IST-2 corresponding to the viscosity fields "b" (black curve), "g" (blue curve), "l" (margenta curve) and "t" (red curve).

Primarily material from the short plate is subducted (see figure 7.11). Since a significant motion of the subduction zone relative to the mantle occurs, the angle of subduction is around 120 degrees.

In many aspects of plate-like behavior model IST-2 is more successful than IST-1: For long episodes the surface velocity is quite uniform. Subduction is asymmetric in terms of different subduction velocities of the plates as well as in terms of the subduction angle. However, one plate is very small and the angle of the subducting material relative to the slower moving surface material is 120 degrees instead of 60 degrees as expected for Earth.

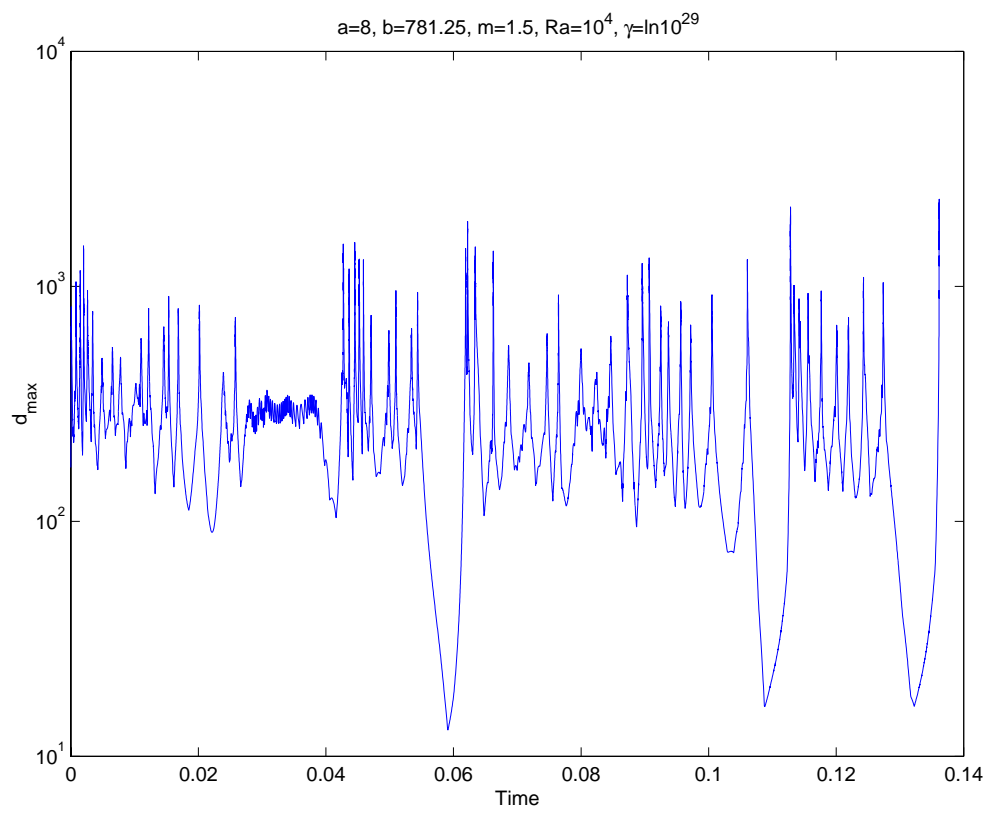

Figure 7.12: Maximum damage versus time for case IST-3 

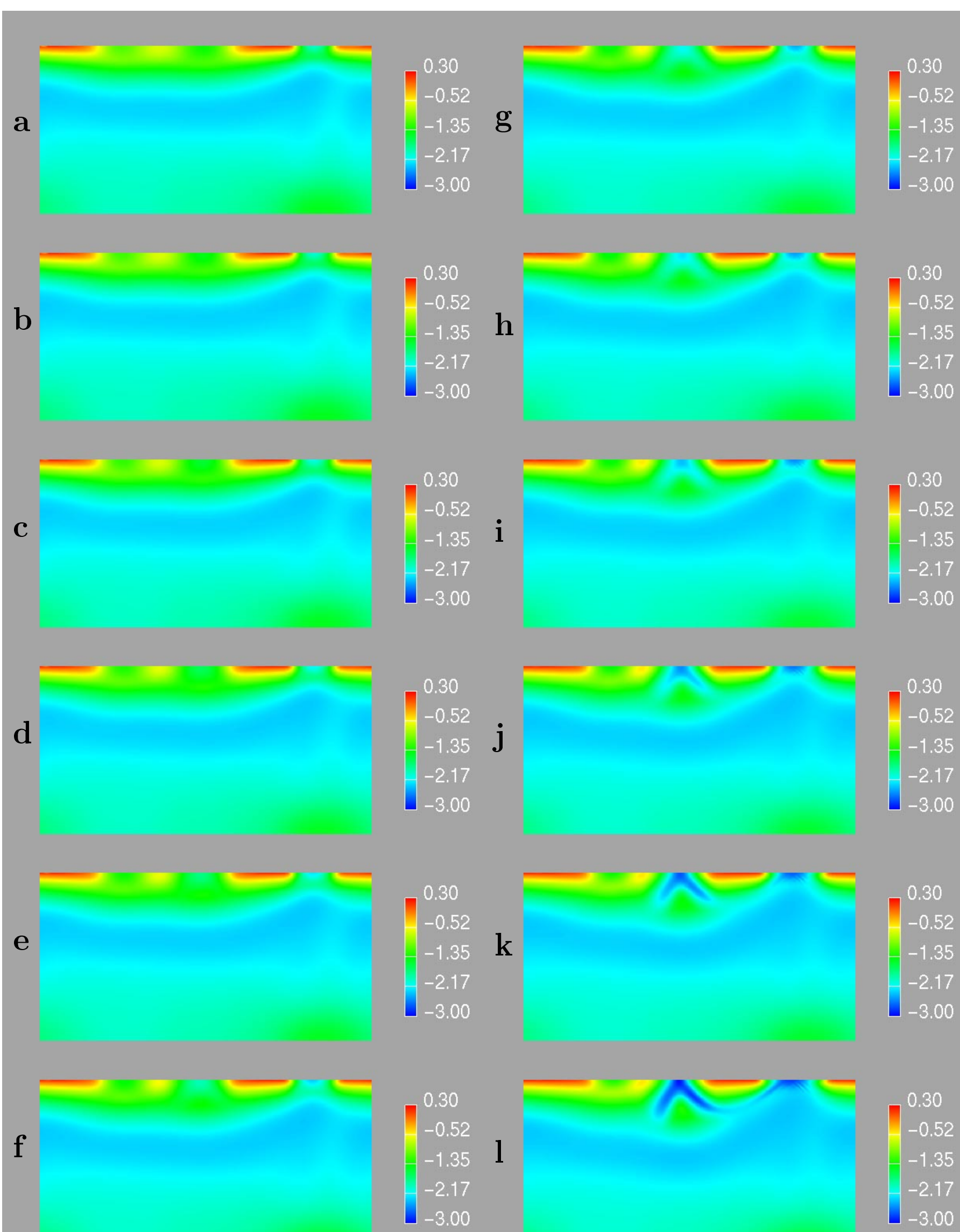


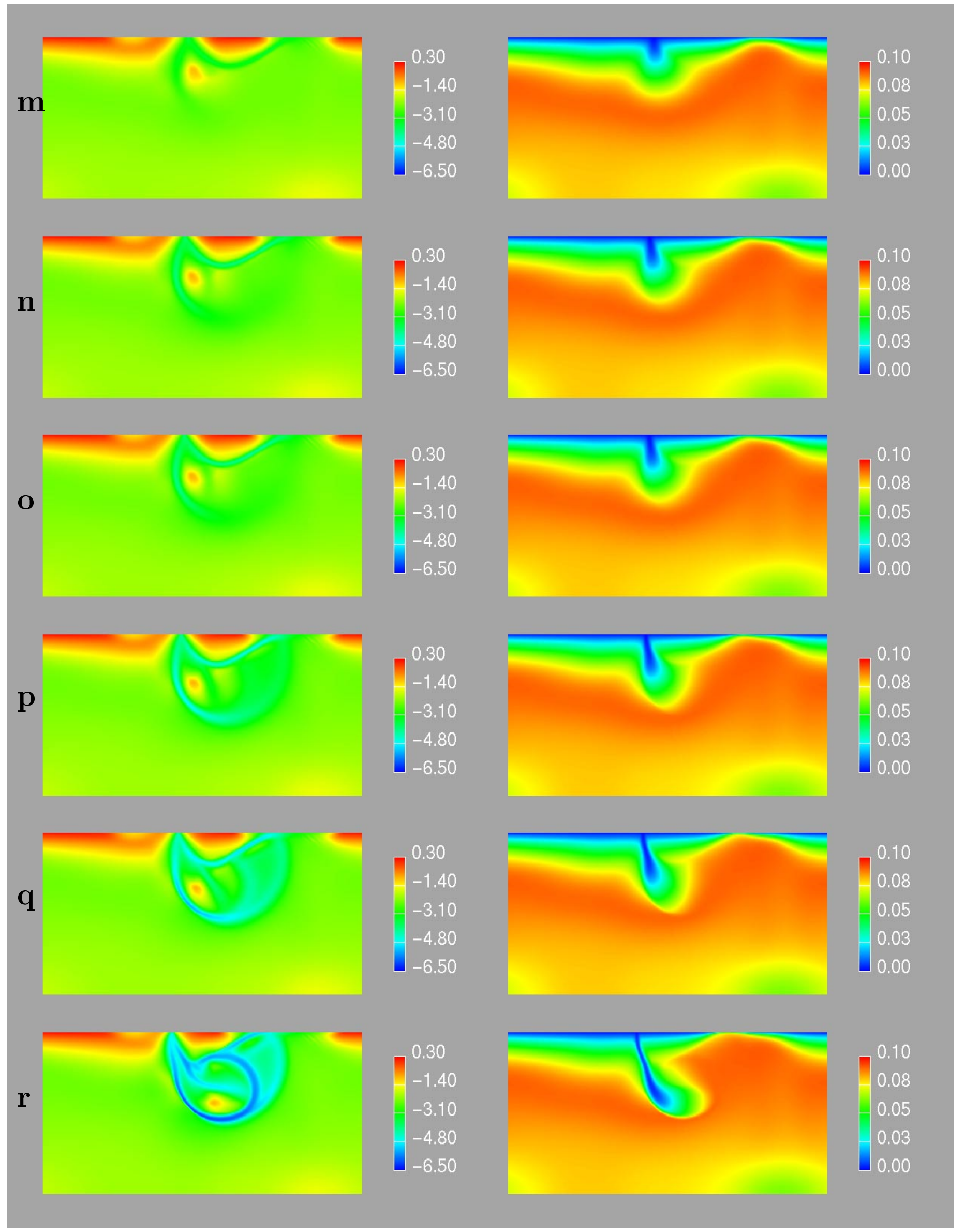


S

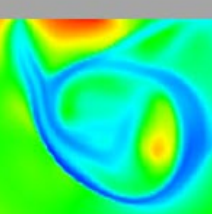

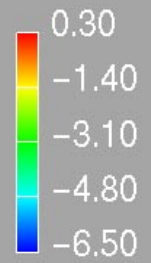

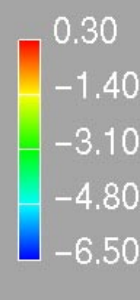

t

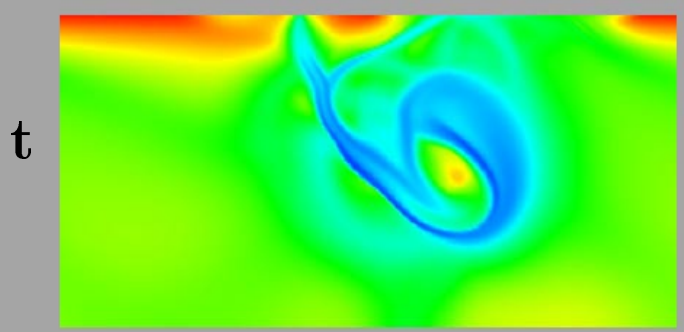

$-$
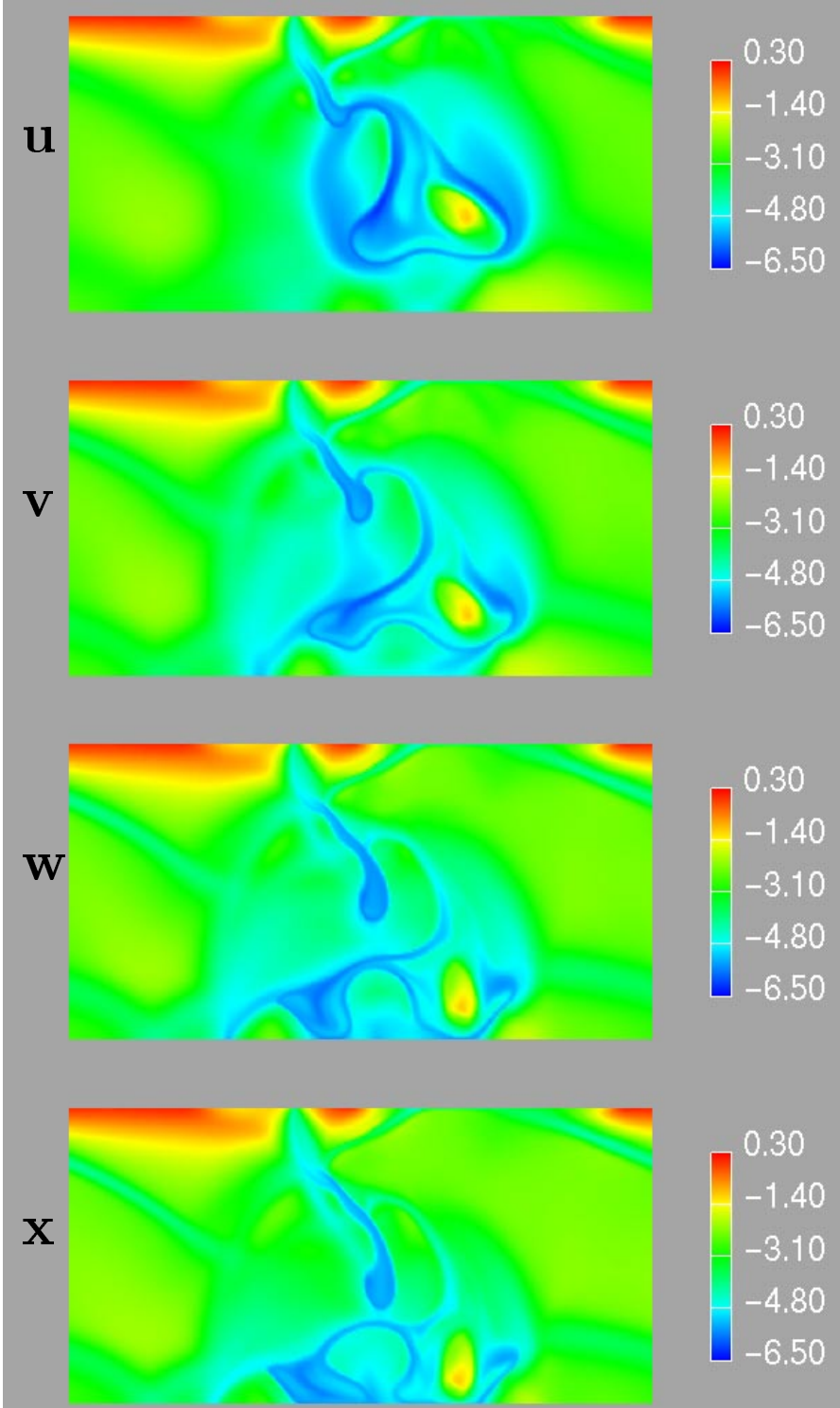
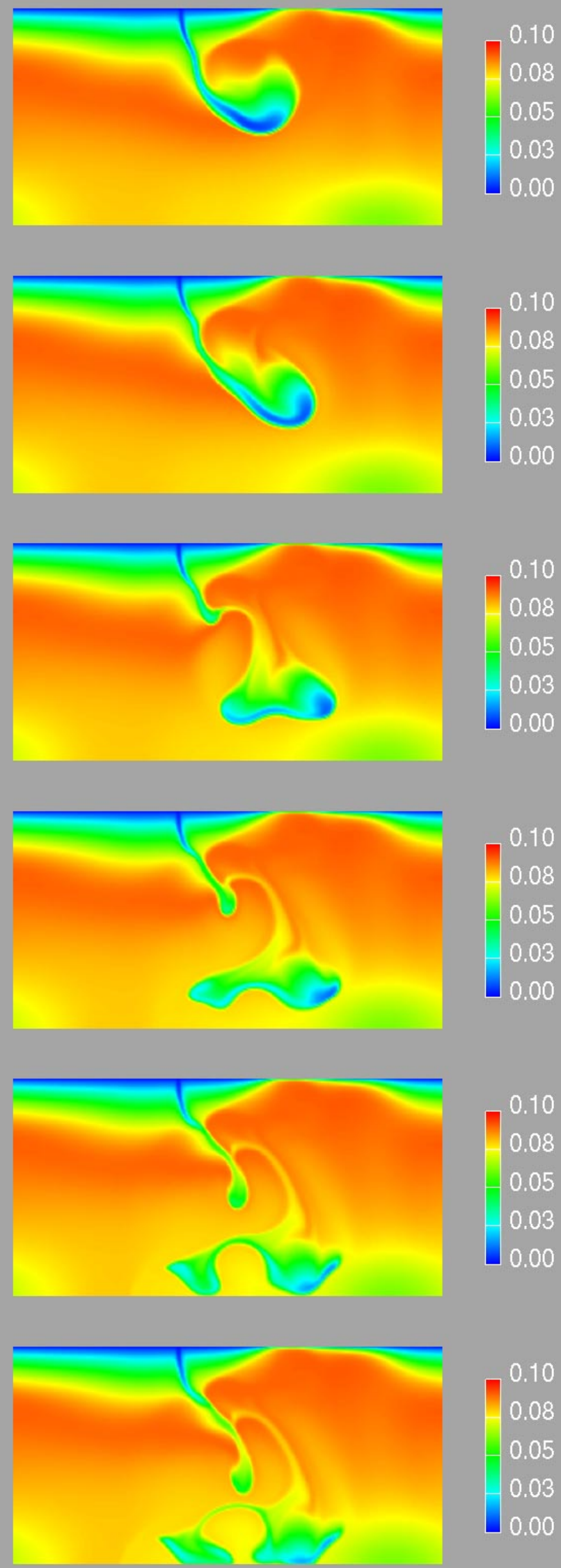
Figure 7.13: Previous page: Viscosity snapshots for case IST-3 between times 0.11090 and 0.11286 (non-equidistant time-steps).

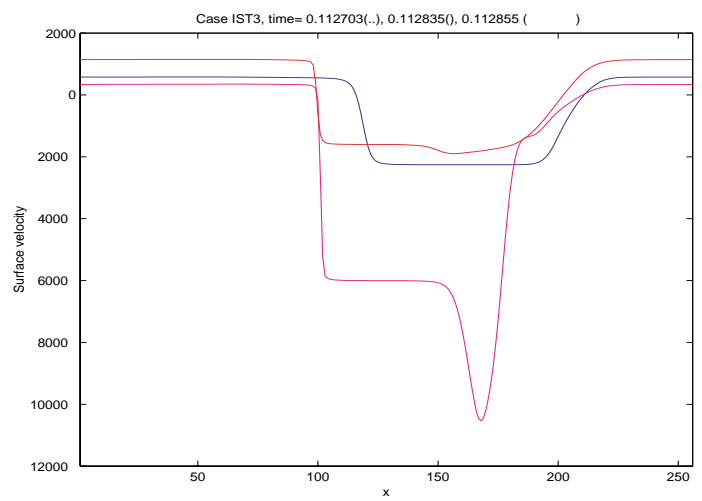

Figure 7.14: Surface velocities for case IST-3 corresponding to the viscosity fields " $m$ " (blue curve), "r" (margenta curve) and " $x "$ (red curve).

Case IST-3 is initiated with fields from IST-2 at the time of 0.3374 . The convection here occurs highly episodic (see figure 7.12). Helpful for explaining this strong time dependence are the viscosity snapshots in figure 7.13. Shown is the development of a new subduction zone and a new passive spreading center (figures 7.13a-l) after a short time of comparatively low activity. One of the subducting plates becomes completely encircled by a LVZ and is now able to move and subduct very fast (figures $7.13 \mathrm{~m}-\mathrm{o}$ ). The fast lateral movement of the plate however triggers the development of a large eddy beneath the plate (figures $7.13 \mathrm{p}-\mathrm{s}$ ). The subducting material is quickly sucked into the eddy and is thus disconnected from the following material (figures 7.13t-x). The velocity of the subducting plate decreases since the slab pull force is reduced (see the surface velocities in figure 7.13). By repeating this process the cold surface material is piecewise and episodically subducted into the mantle.

Although subduction is very asymmetric in this case and the surface velocity is temporary uniform a strongly episodic behavior like this is clearly very non-Earth-like.

\subsubsection{Cases IST-4 and IST-5}

These cases correspond to cases IST-1 and IST-2, however, the source $a$ and the sink $b$ of damage are increased by a factor of 16 .

Case IST-4 is started with the same initial conditions as case IST-1. However, in contrast to IST-1 the episodic subduction of the entire lithosphere (see figure 7.3.2 appears to be a stable convective pattern (compare figures 7.7 and 7.17). A steady state is not reached.

To check whether this result might depend on the initial conditions, IST-4 is run again, starting with the steady state fields obtained in the model IST-1. However, the previous result is confirmed (see figure 7.18 and the viscosity snapshot in figure 7.20).

IST-5 is initiated with a temperature and damage fields from the weakly episodic part of case IST-2. Again convection becomes highly episodic after a short time (see figure 7.19). Figure 7.20 shows the viscosity field at the time the calculation had to be ended because of 

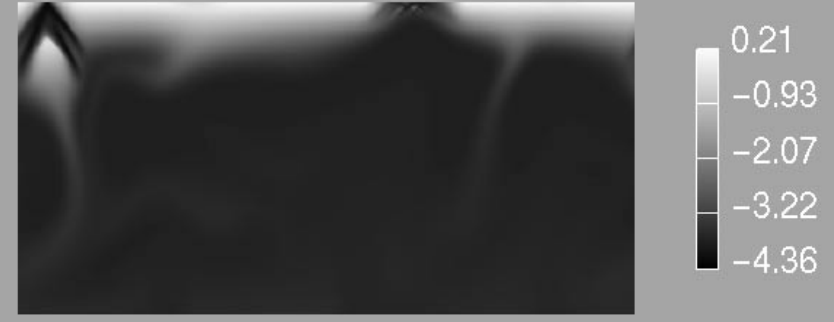

d

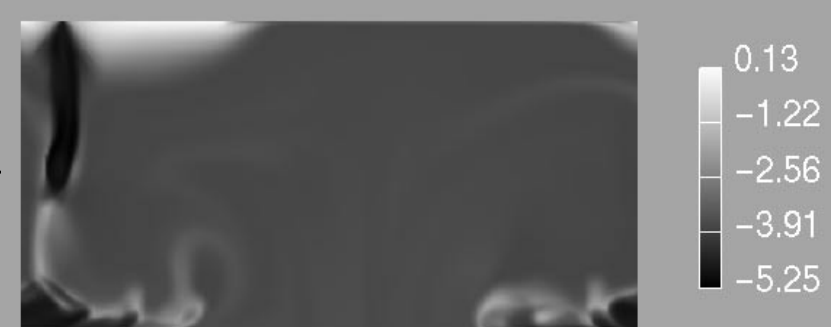

b
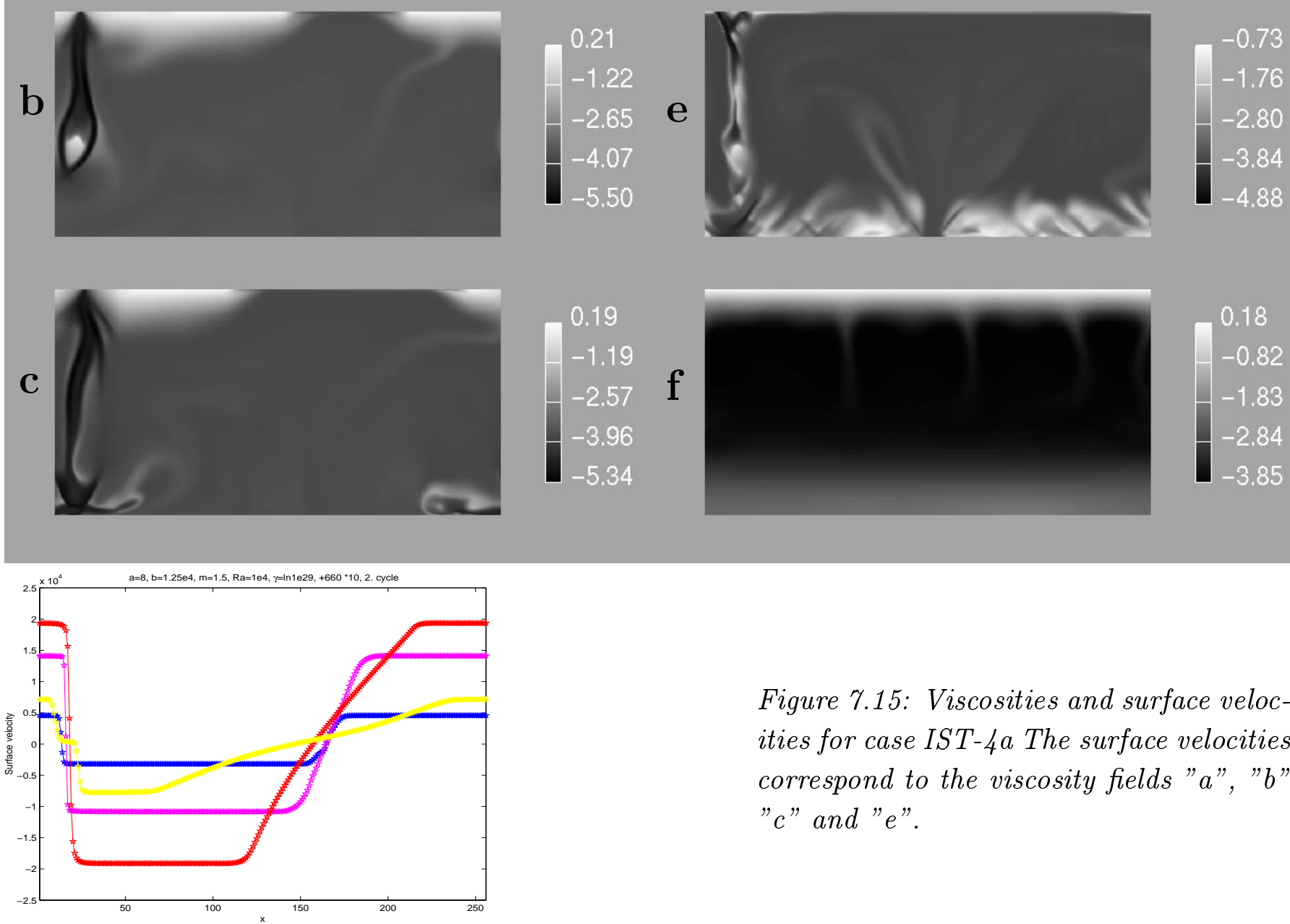

Figure 7.15: Viscosities and surface velocities for case IST-4a The surface velocities correspond to the viscosity fields " $a$ ", " $b "$ "c" and "e".

numerical difficulties.

In summary, the highly episodic convection in these models appears again very non-Earthlike.

\subsubsection{Summary and Discussion}

The effects of variations in the input parameters $a$ (source of damage) and $b$ (sink of damage) are basically the same as observed in the previous chapters:

(a) An increase of the damage source $a$ increases the asymmetry of the subduction but supports episodic convection pattern. Moreover the size of the predominately subducting plate 


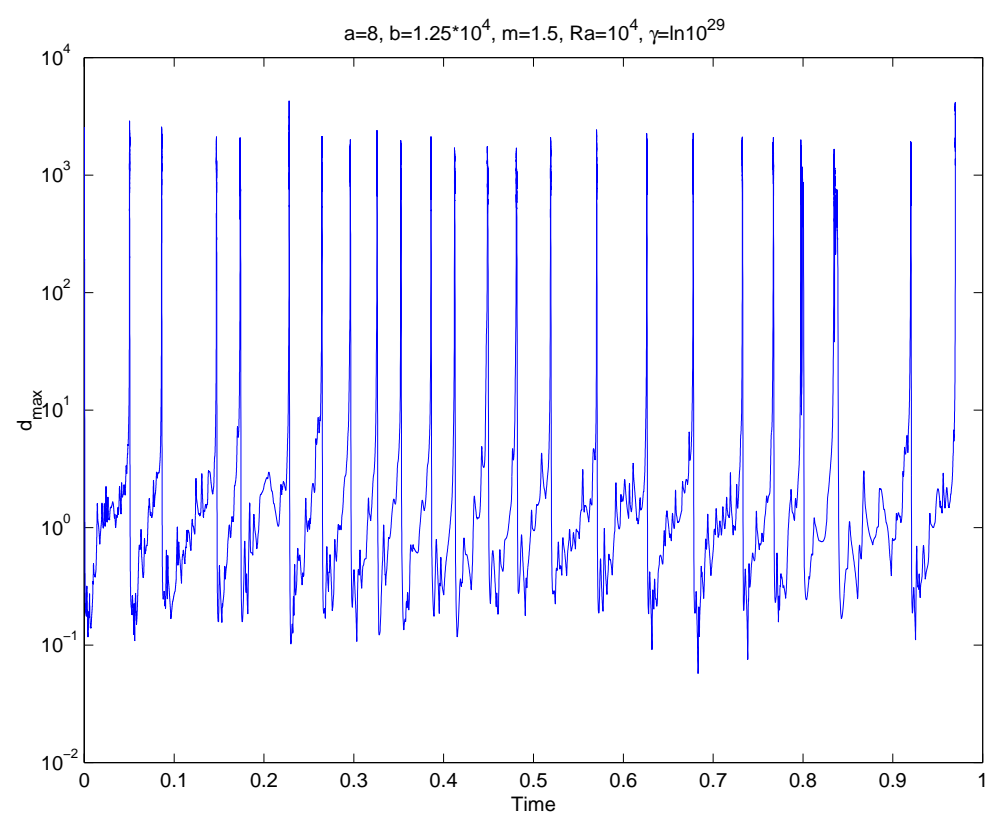

Figure 7.16: Maximum damage versus time for case IST-4a

tend to decrease with increasing $a$.

(b) The simultaneous increase of the source $a$ and the sink $b$ of damage produces more focused, "v"-shaped LVZs and therefore increases the uniformity in the motion of the surface material. However, it also enforces a stronger time-dependence and episodicity of the convective structures in the box.

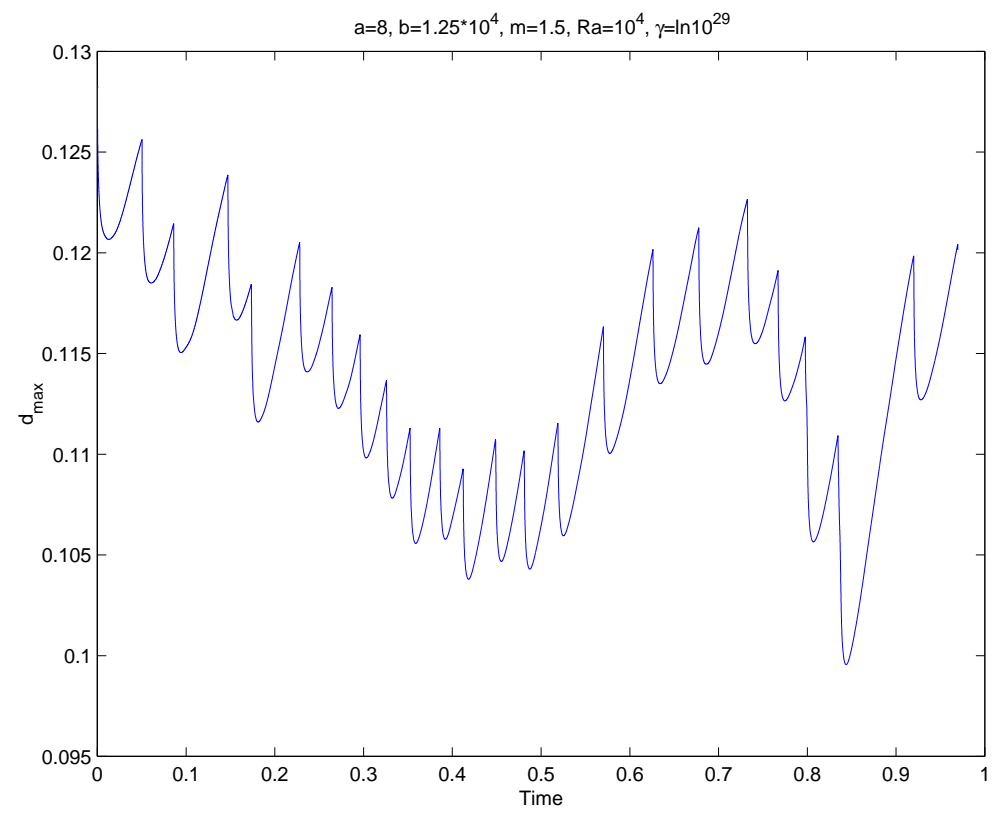

Figure 7.17: Averaged temperature versus time for case IST-4a 


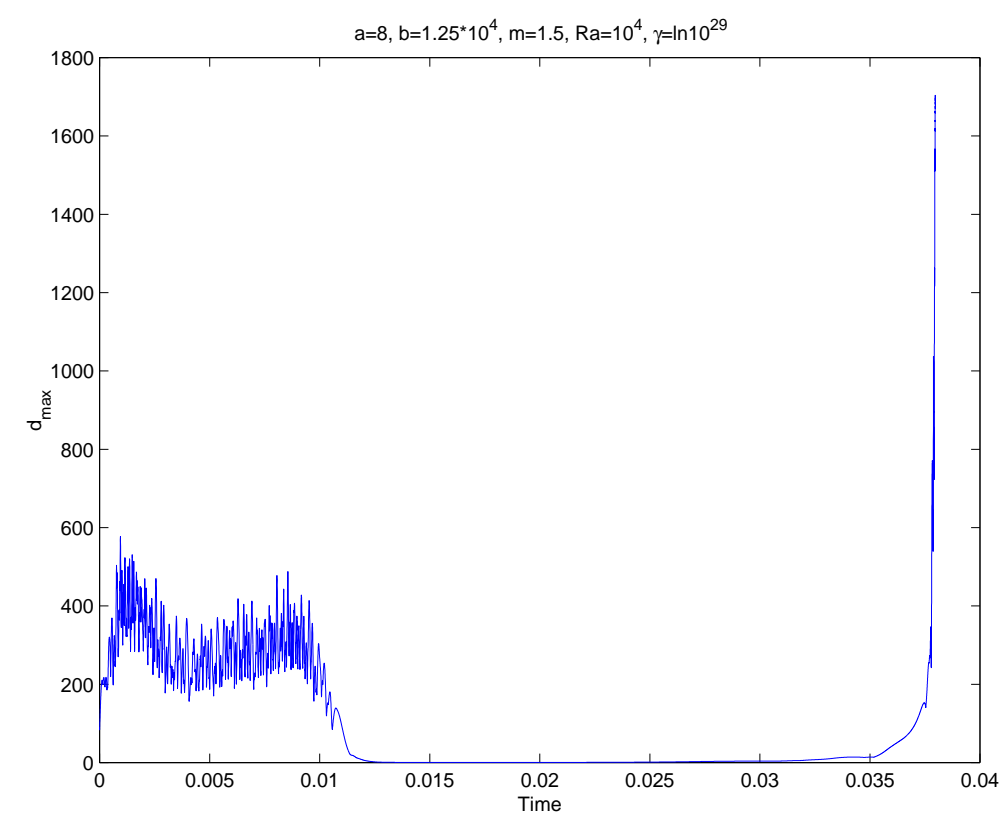

Figure 7.18: Maximum damage versus time for case IST-4

In order to understand, how plate-like behavior in simulations can be obtained and which processes are important to reproduce terrestrial plate tectonics the following points are relevant:

(1) Cases IST-2 and IST-3 are good examples to qualitatively understand how asymmetry of subduction develops in my model. The important point is that at the beginning of the

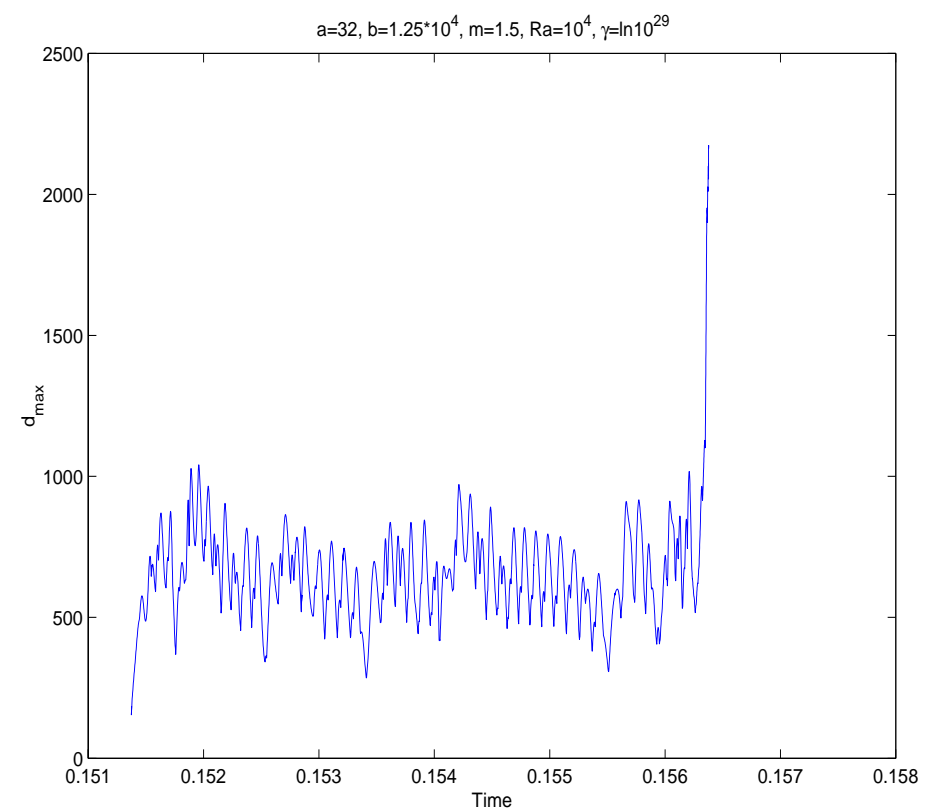

Figure 7.19: Maximum damage versus time for case IST-5 


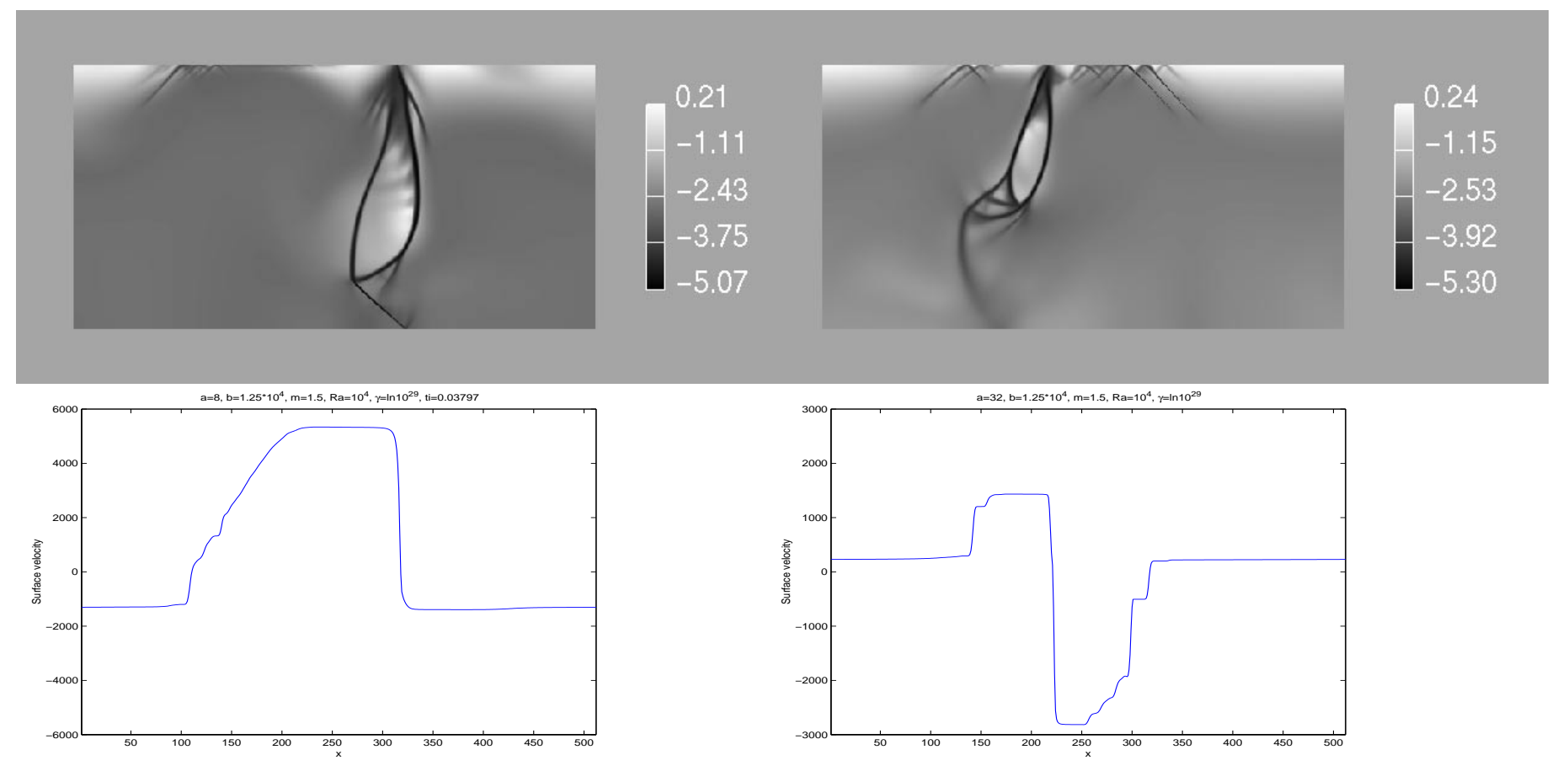

Figure 7.20: Left frames: Steady state viscosity and surface velocity for case IST-4 $(a=8$, $\left.b=1.25 \cdot 10^{4}, m=1.5, R a=10^{4}, \gamma=\ln 10^{29}\right)$, right frames: The same for IST-5 $(a=32$, $\left.b=1.25 \cdot 10^{4}, m=1.5, R a=10^{4}, \gamma=\ln 10^{29}\right)$.

subduction process (figure 7.13a) the lithospheric structure and the overall convection pattern within the box are very symmetric. However, one of the newly developing plates is a little smaller than the other one. The self-focusing mechanism in my rheology is able to increase those small differences: A LVZ develops around the smaller plate, but not around the larger one. The smaller plate is basically decoupled from the underlying mantle material and moves quickly towards the subduction zone, while the larger plate remains nearly immobile. The large-scale flows in the box are necessarily affected and become structured in a way that keeps the subduction of the smaller plate going even if its negative buoyancy is significantly lower than the buoyancy of the immobile plate. Moreover, the large-scale flows also influence the direction of the propagation of the lithospheric material into the mantle. In simulation IST-3 subduction angles of about 120 degrees are observed. Since around 60 degrees are usual for the Earth this is an observation not in favor of my model. IST-2 however demonstrates the importance of a relative motion between mantle and lithosphere for a subduction angle different from 90 degrees.

(2) In the last section I presented some first estimates for the minimum value of the damage source parameter $a$ necessary to break a stagnant lid. The results discussed here may also suggest an upper boundary for $a_{\text {Earth }}$ : To produce asymmetry in subduction $a_{\text {Earth }}$ should be higher that $a_{\text {break,Earth }}$ (see case IST-2), however, highly episodic subduction is to avoid (see case IST-3). A first estimate for an upper boundary for $a_{E a r t h}$ is therefore

$$
a_{\text {upper,Earth }, 1}<6 \cdot a_{\text {break,Earth }}
$$


(3) The fundamental problem with trying to obtain plate-like behavior using this rheology is that each variation of the model parameters produces a positive effect in terms of plate tectonics as well as a negative one. Increasing $a$ increases asymmetry but reduces the plate size and increases episodicity, increasing $a$ and $b$ focus the LVZs but also promotes episodic convection. A model including all aspects of terrestrial plate tectonics therefore seems difficult to obtain with this rheology.

Since some limits of my rheology were also obtained from the estimation of $a_{b r e a k, \text { Earth }}$ and $b_{\text {break,Earth }}$ in the last section an extension of my rheology seems necessary.

\subsection{Depth-dependent viscosities}

\subsubsection{Introduction}

The previous sections I have demonstrated that the rheological model used is probably not able to reproduce all features of terrestrial plate tectonics. The basic problem is the very episodic behavior which is obtained when either the damage source $a$ is increased to promote the asymmetry of subduction or when source $a$ and sink $b$ are simultaneously increased to focus the LVZs.

For yield stress rheologies similar situations are improved by including a depth-dependence of viscosity into the model (see Stein 2000). The increase of viscosity with depth basically inhibits the very fast subduction of large amounts of material into the deeper mantle.

In this section the effect of three types of viscosity depth-dependences are tested using cases IST-4 $\left(a=8, b=1.25 \cdot 10^{4}, m=1.5, R a=10^{4}, \gamma=\ln 10^{29}\right)$ and IST-5 $(a=32$, other parameters identical) as reference cases. Table 7.3 shows an overview over the cases presented here.

\begin{tabular}{l|c|c|r|c|l} 
Series & Case & Aspect Ratio & $a$ & Depth-dependence & Regime \\
\hline \multirow{6}{*}{ DD } & 1 & 2 & 8 & none & VDR VI \\
& 2 & 2 & 8 & $\zeta=\ln 10$ & VDR II \\
& 3 & 2 & 8 & $\zeta=\ln 100$ & VDR II - HDR \\
& 4 & 2 & 8 & Jump factor 10 & VDR II - HDR \\
& 5 & 4 & 8 & none & VDR VI \\
& 6 & 4 & 8 & $\zeta=\ln 100$ & VDR II - HDR \\
& 7 & 4 & 8 & Jump factor 10 & VDR II - HDR \\
& 8 & 4 & 32 & Jump factor 10 & HDR
\end{tabular}

Table 7.3: Overview over the parameter studies in the section 7.4. 


\subsubsection{Cases DD-1 - DD-4}

The differences between the four cases DD1-DD4 are presented in the figures 7.21 - 7.23. An exponential increase of the viscosity depth-dependence (cases DD-2 and DD-3) basically inhibits the episodic subduction of the entire lithosphere as expected. Even for a weak depthdependence (case DD-2) a significant increase in the length of the "active" periods within the variable damage regime is visible (figure 7.21). For higher $\zeta$ (case DD-3) a statistical steady state is already obtained at the end of the second episodic overturn. A permanent subduction zone develops and a passive spreading center is always present, however its position varies and causes a weak time-dependence of the simulation. The permanent existence of a passive spreading center also promotes the heat transport out of the system (figure 7.22 ). The averaged temperature in the box quickly decreases to about $2 / 3^{\text {th }}$ of case DD- 1 , reaching a similar level than in case IST-1 (case DD-1 with lower $a$ and $b$, see last section).
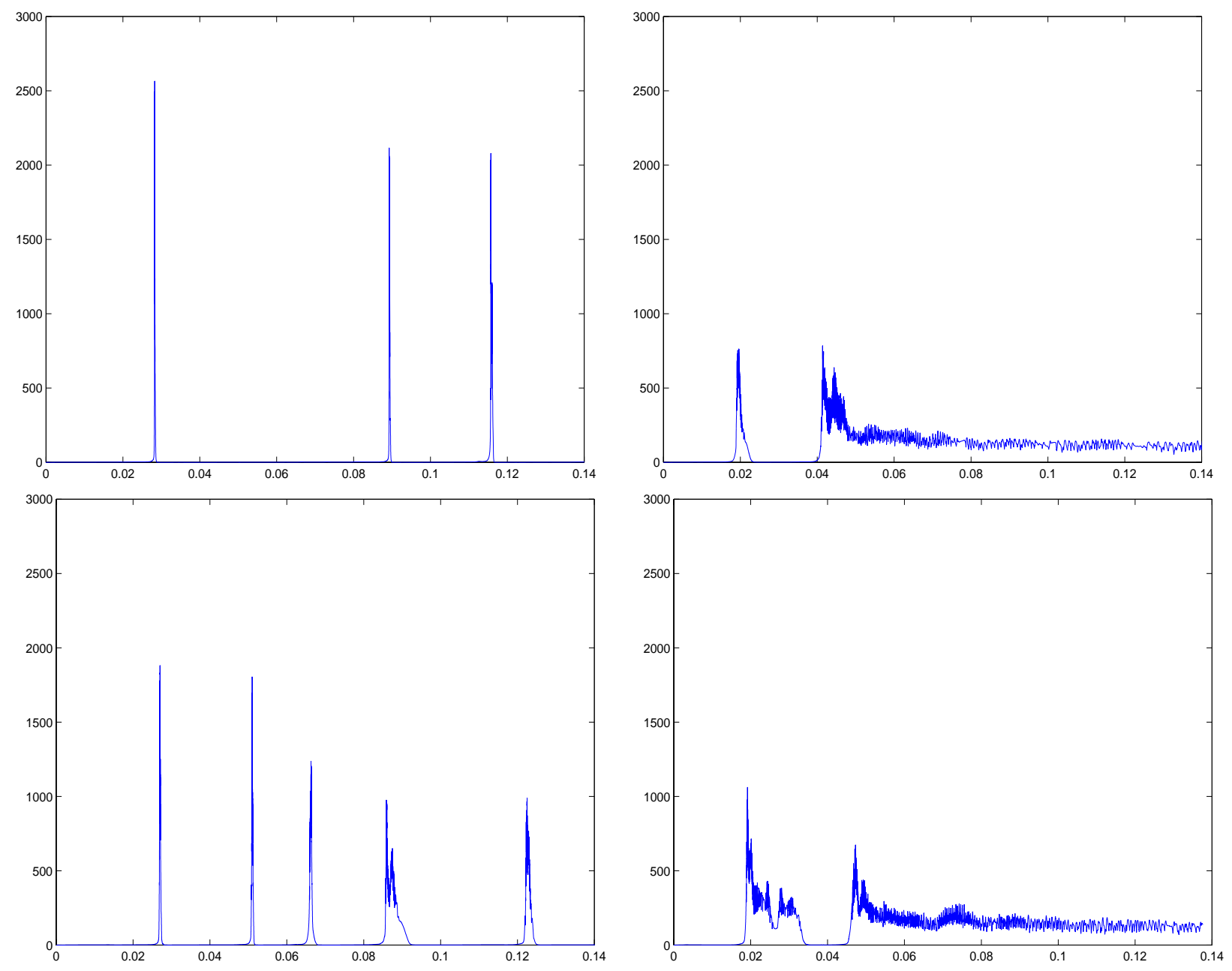

Figure 7.21: Maximum damage versus time for case $D D-1$ (top,left), DD-2 (with $\zeta=10$; bottom, left), DD-3 (with $\zeta=100$ top, right) and DD-4 (with a viscosity jump by a factor of ten at the $660 \mathrm{~km}$ phase transition; bottom, right) 


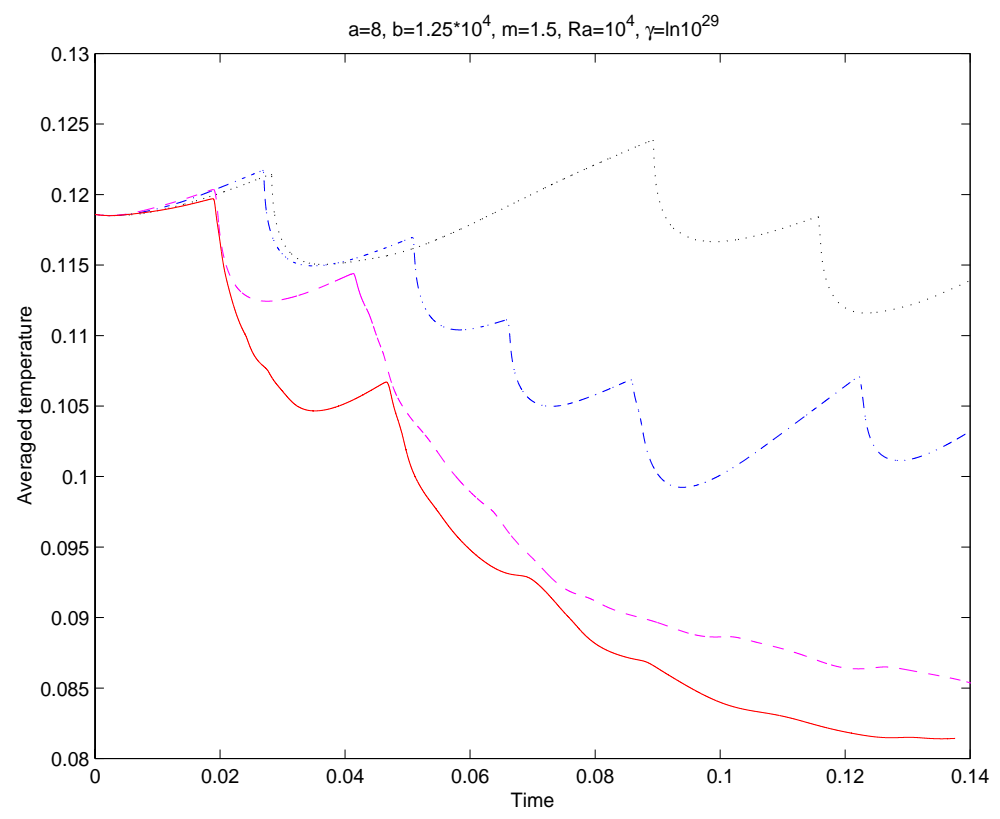

Figure 7.22: Averaged temperature versus time for DD-1 (dotted curve), DD-2 (dashed and dotted curve), DD-3 (dashed curve) and DD-4 (solid curve).
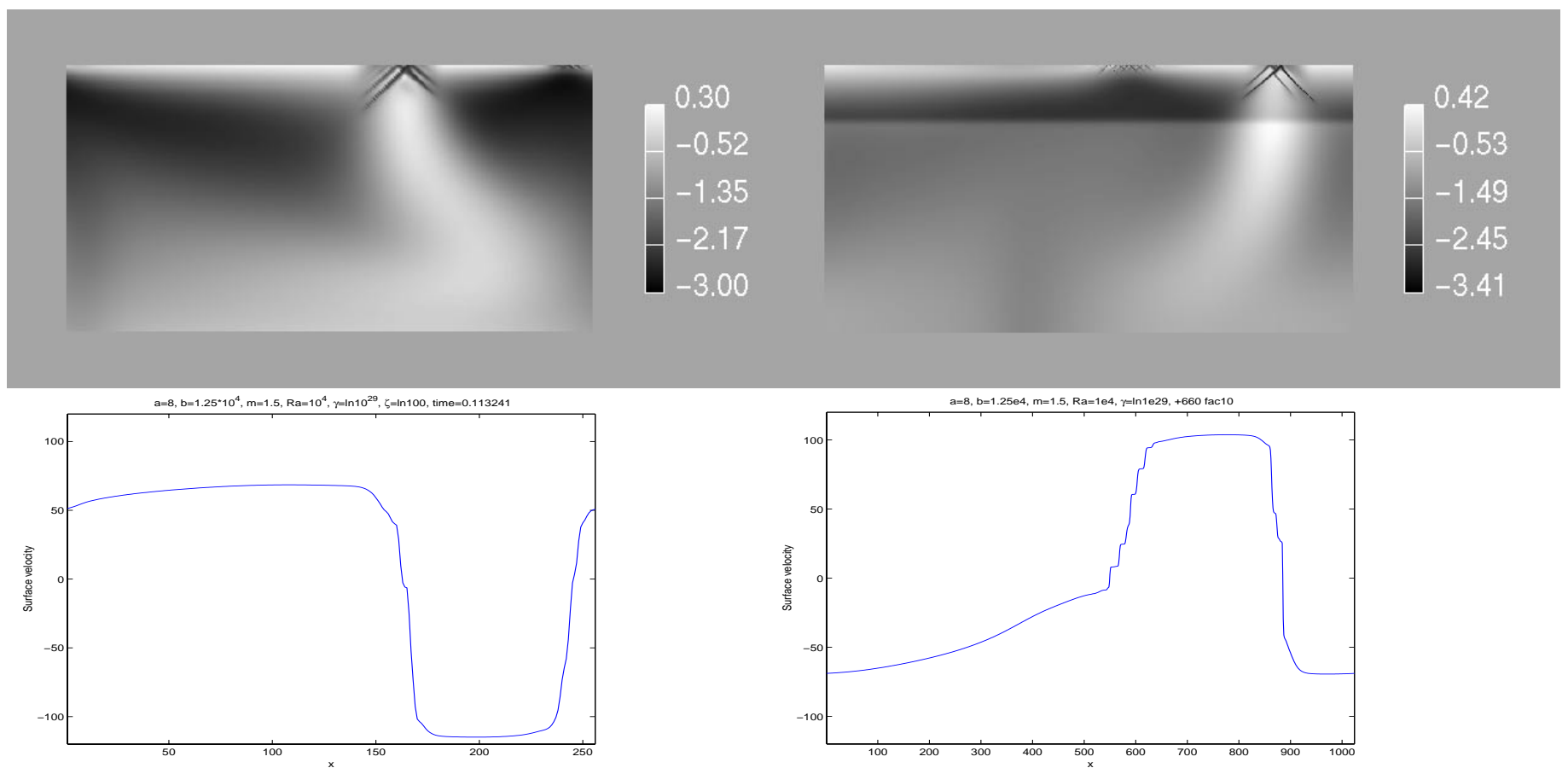

Figure 7.23: Steady state viscosities and surface velocities for cases DD-3 (left frames, exponential viscosity increase by a factor of hundred) and DD-4 (right frames, viscosity jump by a factor of ten at the $660 \mathrm{~km}$ discontinuity) 
The plate-like behavior for case DD-3 is naturally much better than for case DD-1 since the episodic vanishing of the entire lithosphere stops. Compared to case IST-1, the LVZs and the surface velocity variations are much more localized (surface velocities in figure 7.23). The two plates move with different velocities in the same order of magnitude as in IST-1, but around one order of magnitude slower than on Earth. The subduction angle varies in time between about 80 and 100 degrees.

A viscosity jump at the $660 \mathrm{~km}$ discontinuity by a factor of ten (case DD-4) produces very similar results than case DD-3.

\subsubsection{Cases DD-5 - DD-7}

Cases DD-3 and DD-4 are quite successful in reproducing many features of terrestrial plate tectonics. However, the computational domain in these cases is too small to find out whether very extended plates can be obtained with the depth-dependent viscosity. Therefore cases DD-1, DD-3 and DD-4 are repeated in a box of aspect ratio four.

Case DD-5 (without depth-dependent viscosity) uses initial conditions similar to the first calculation in DD-1. It also shows episodic behavior. However, the "active" episodes are longer than in case DD-1 since only one downwelling is present at the same time (see figures 7.24 and 7.25) ). The subduction of the entire lithosphere therefore takes longer than in case DD-1 (similar to cases ISL-1 and ISL-2 in the previous chapter).

Both cases DD-6 and DD-7 start with similar initial conditions as cases DD-3 and DD-4. The temporal evolutions of the damage or the averaged temperature are qualitatively identical to those for cases DD-3 and DD-4 and are therefore not presented here. An obvious difference between DD-6 and DD-7 is that DD-7 develops a two cell convection with extended plates much earlier than DD-6 which shows four convection cells for a significant time (see figures

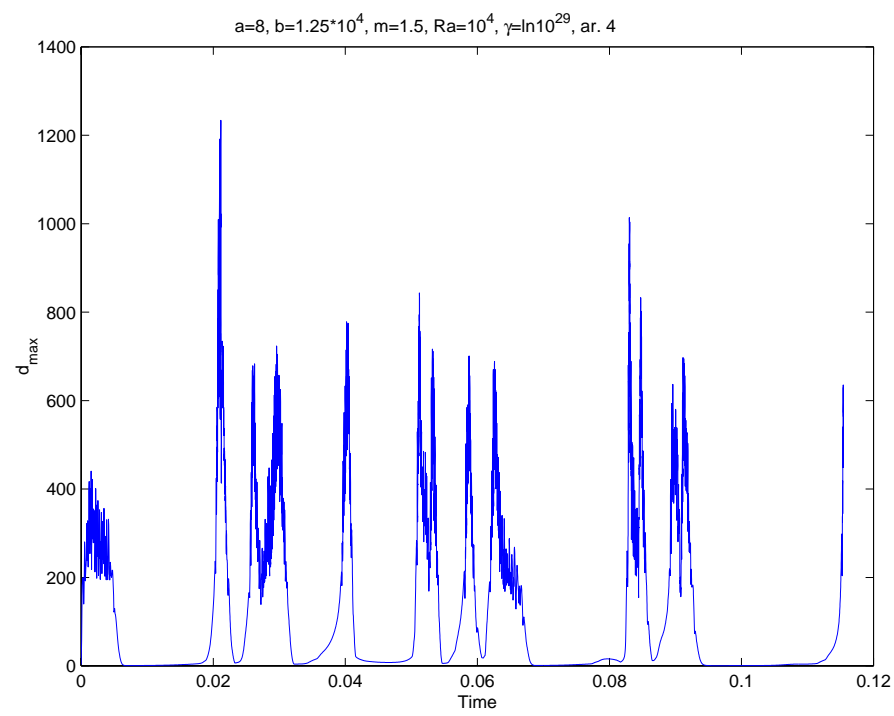

Figure 7.24: Maximum damage versus time for case DD-5 


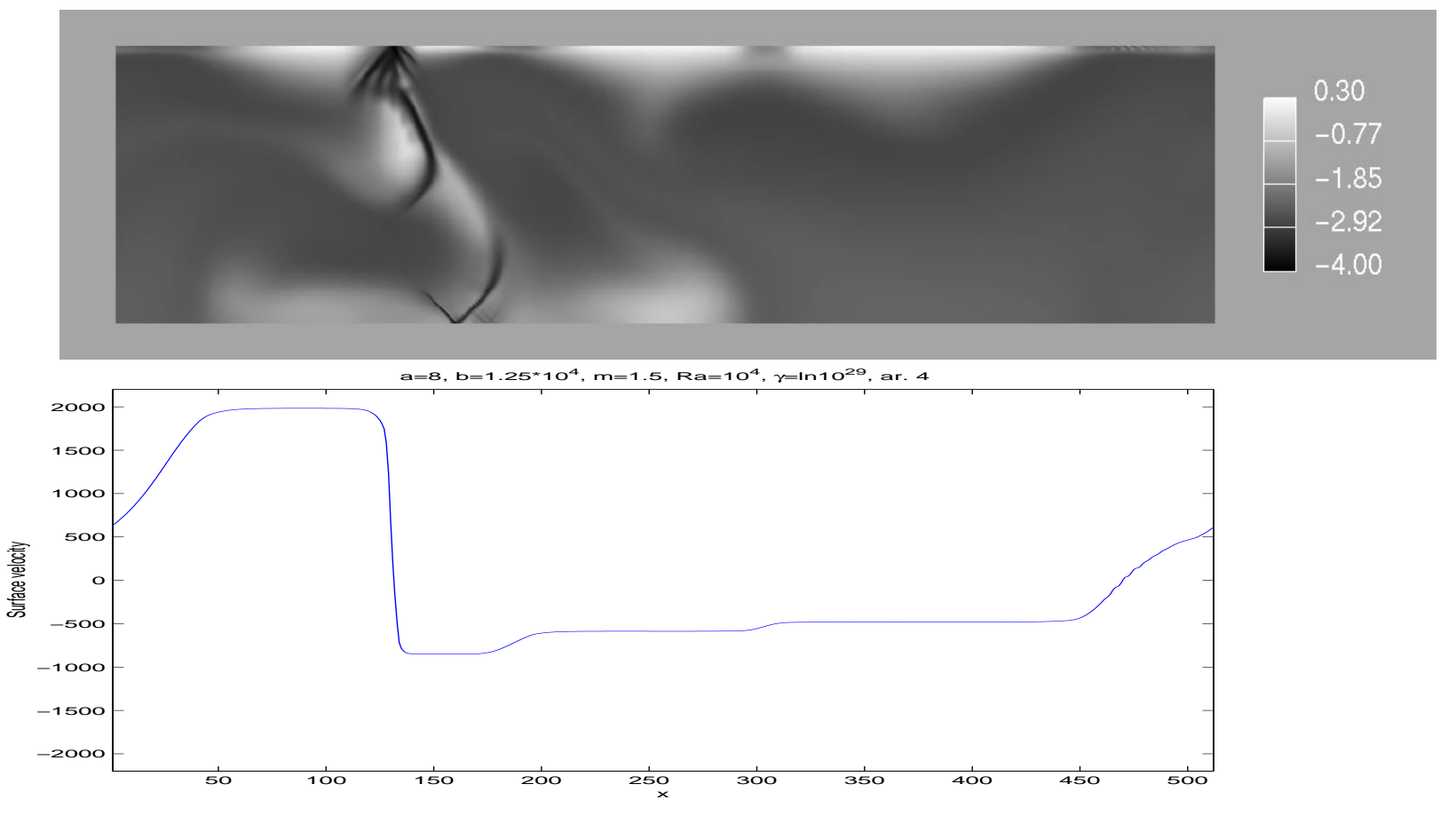

Figure 7.25: Viscosity and surface velocity snapshot for case DD-5 at the beginning of a new vigorous subduction cycle.

7.26 and 7.27). A viscosity jump at the $660 \mathrm{~km}$ discontinuity might therefore be more appropriate to create extended plates in numerical simulations than an exponential increase of viscosity.

\subsubsection{Case DD-8}

The introduction of a viscosity jump at the $660 \mathrm{~km}$ discontinuity obviously inhibits the episodic subduction of the entire lithosphere and allows the evolution of extended plates. However, the asymmetry of subduction is not satisfying. To increase the asymmetry the damage source parameter $a$ is now increased by a factor of four compared to case DD-7. The initial conditions are taken from case IST-2. Remembering cases IST-1 and IST-2 a significant increase in the asymmetry of subduction, but also a decrease in the size of one plate is expected.

Surprisingly convection in this model appears quite similar to case DD-7 (compare figures 7.26 and 7.29). Both cases show little asymmetry of subduction and two extended plates for long time-intervals (see figure 7.29a). However, these plates are obviously weaker here than in case DD-7: While usually just one passive spreading center is present in case DD-7, case DD- 8 shows two or three of them for extended times (figure 7.29b). The time-dependence of case DD-8 is therefore more developed than that for DD-7 (see figure 7.28). 


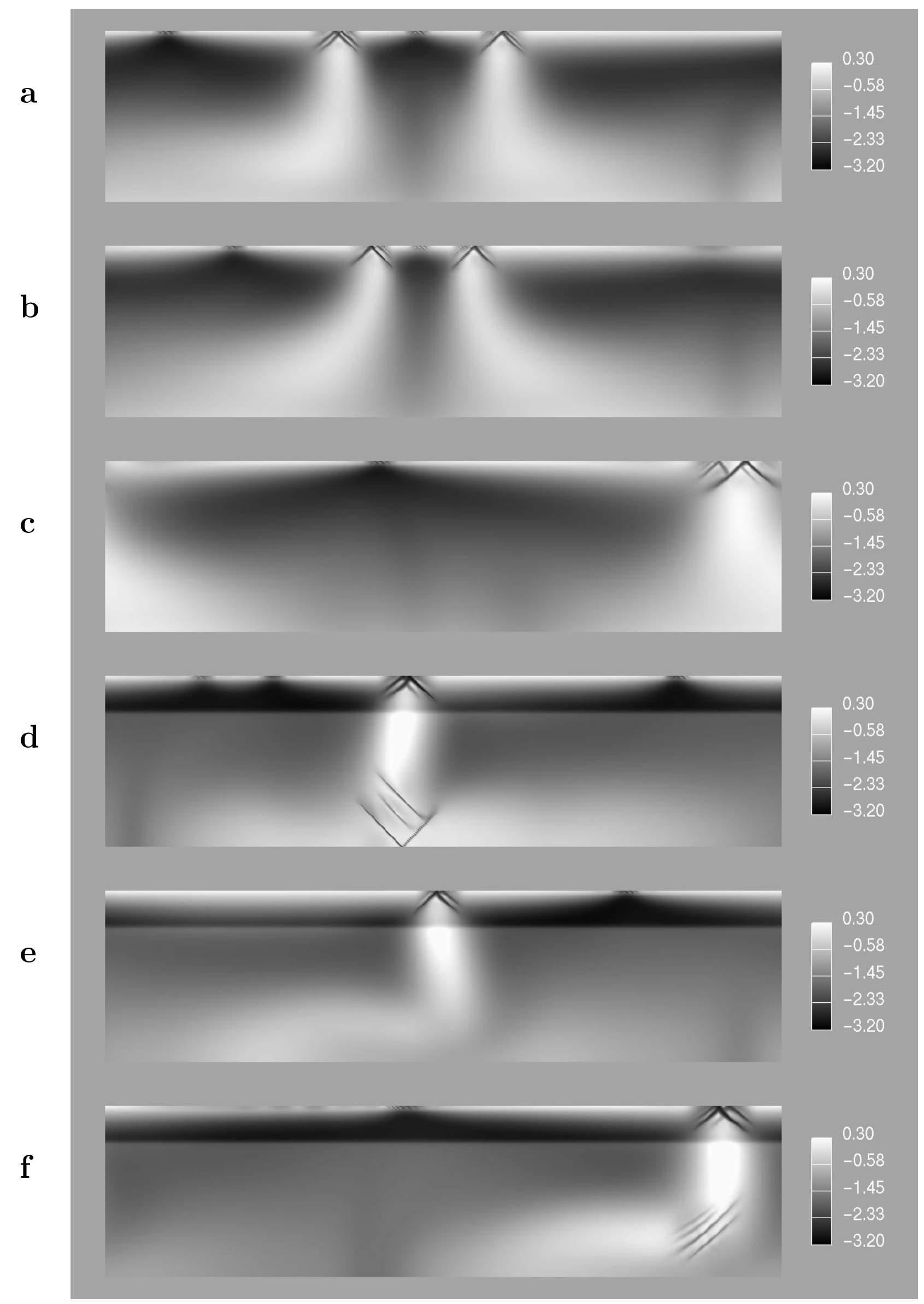



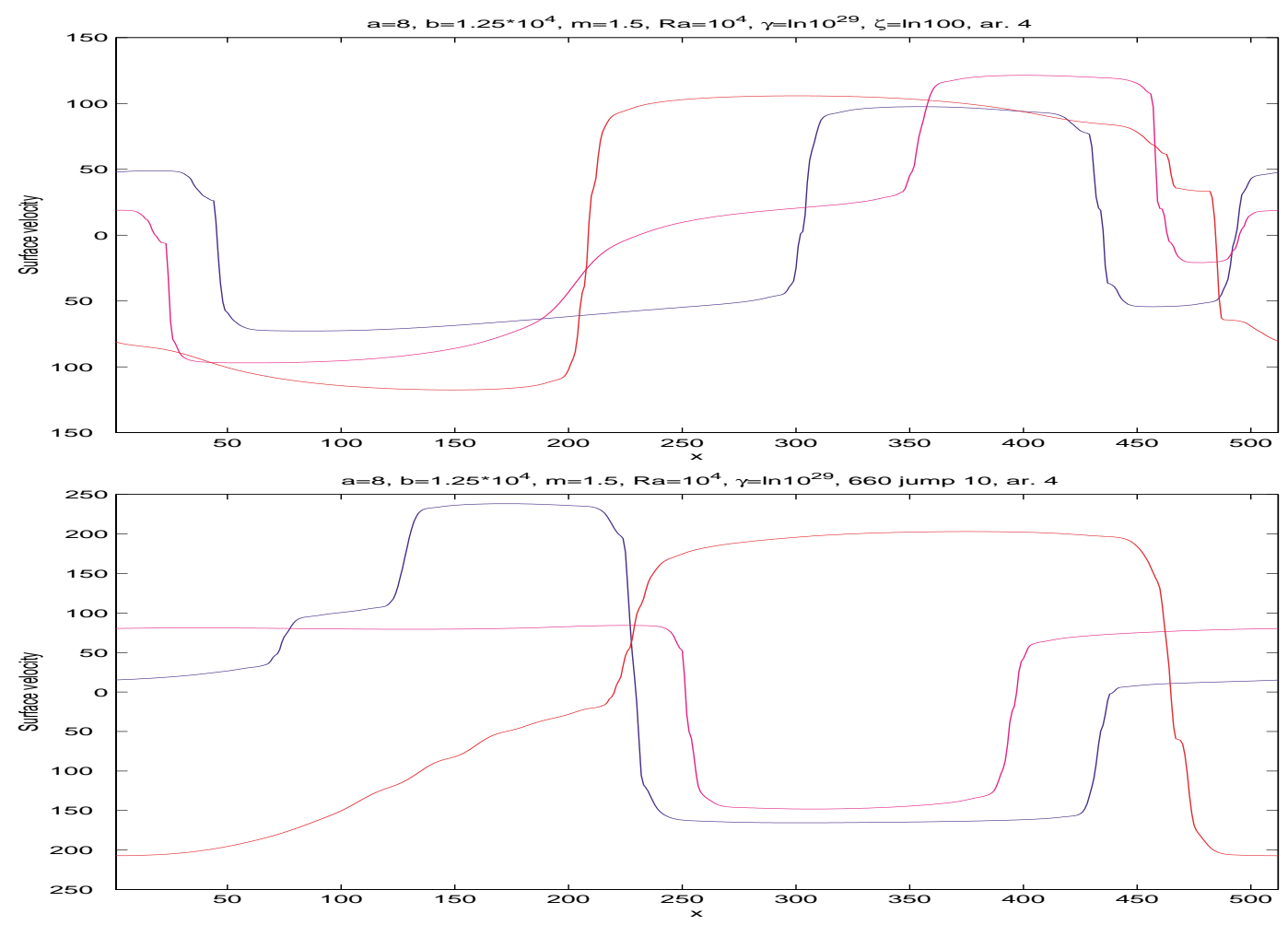

Figure 7.26: Previous page: Viscosity snapshots for case DD-6 at times $0.1084,0.1205$ and 0.1523 (frames a-c) and for case DD-7 at the same times. This page: The corresponding surface velocities. The blue curves belongs to frames a $(d)$ the margenta curves to $b(e)$ and the red curves to $c(f)$.

In summary, an increase of the damage source parameter $a$ influences the overall convection pattern within the box significantly weaker with an additional depth-dependence of viscosity
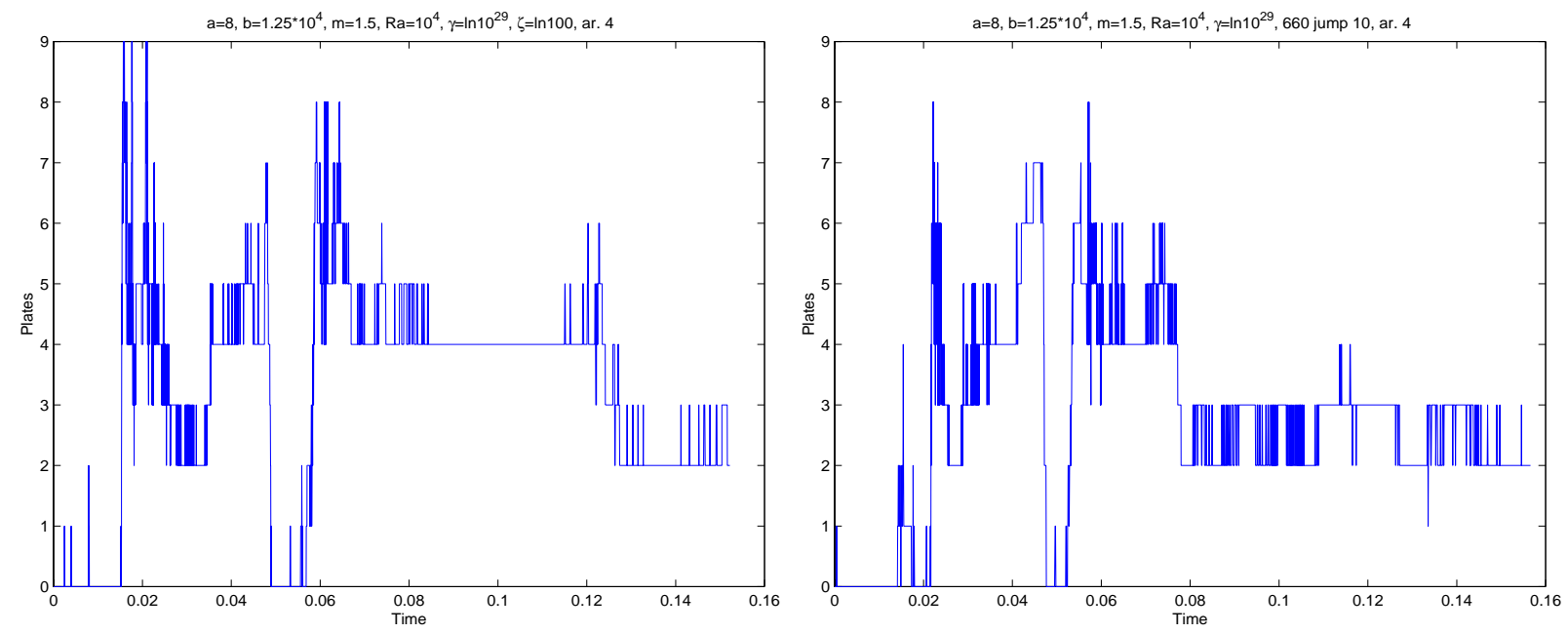

Figure 7.27: Number of plates versus time for cases DD-6 and DD-7 


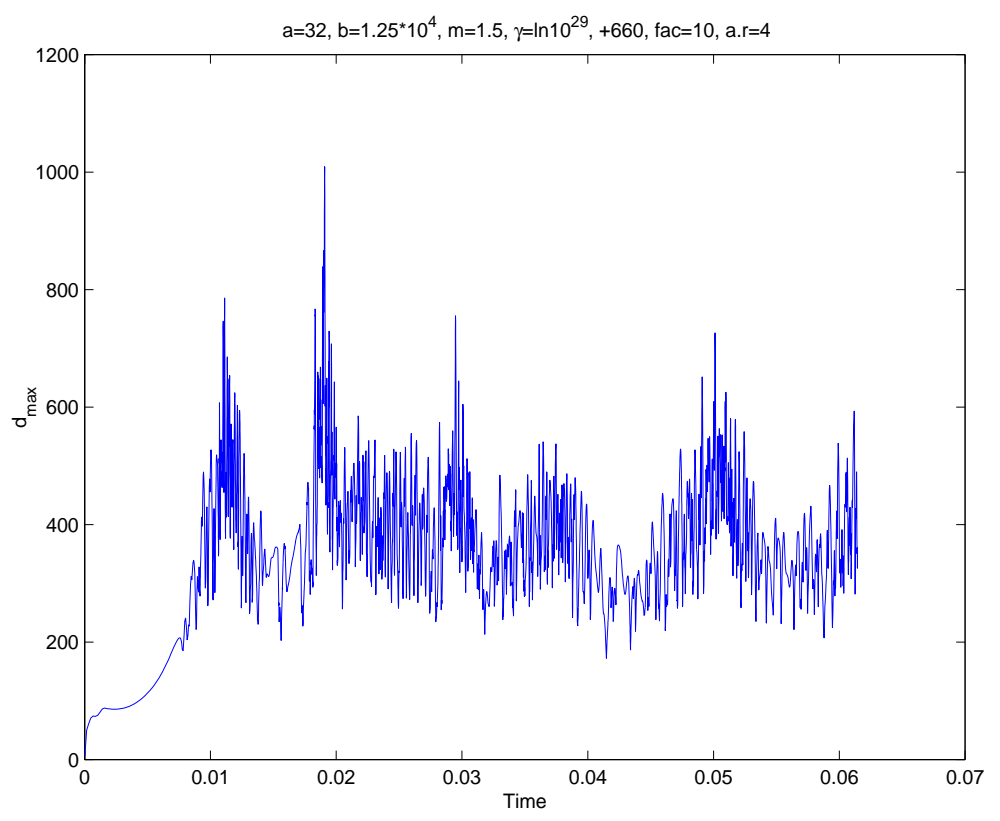

Figure 7.28: Maximum damage versus Time for case DD-8
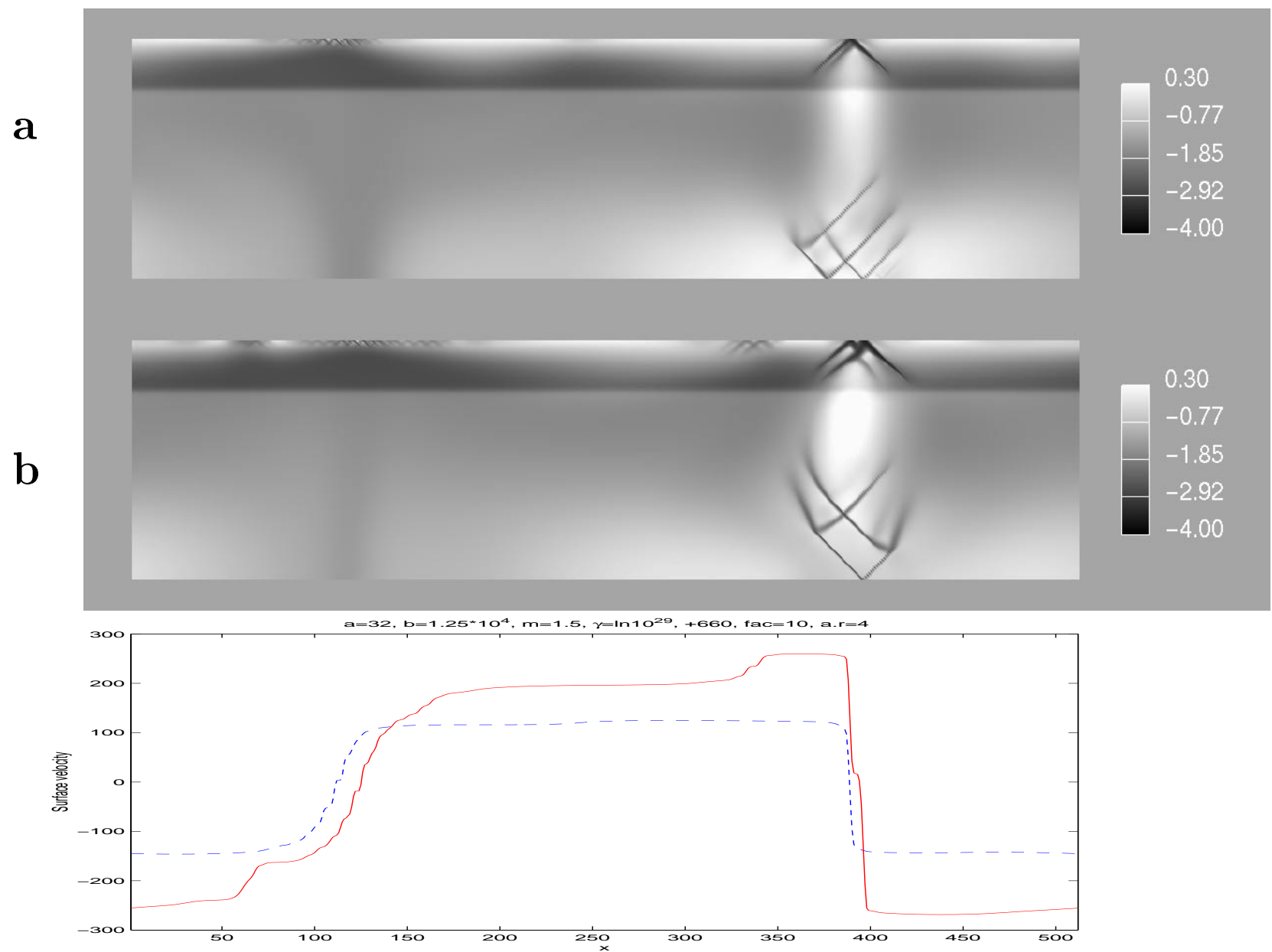

Figure 7.29: Viscosity and surface velocity for case DD-8 at times 0.05545 and 0.06145 . 
than without that depth-dependence.

\subsubsection{Case DD-9}

I finally present a calculation with increased Rayleigh number to demonstrate the good plate like behavior of my model even for more vigorous convection.

The parameters used in this case DD-9 are $a=1, b=5 \cdot 10^{4}, m=1.5, R a=10^{5}, \gamma=\ln 10^{50}$ and a viscosity jump at the $660 \mathrm{~km}$ discontinuity by a factor of ten. The aspect ratio is four. Initial conditions are taken from the corresponding simulation in a smaller box (aspect ratio two) without the viscosity jump. This previous simulation shows episodic convection similar to case DD-1.

Figure 7.30 presents the temporal evolution of the model at the beginning of the simulation. In the time-interval between $0.65 \cdot 10^{-3}$ and $1.4 \cdot 10^{-3}$ thick lithosphere is episodically subducted forming one subduction zone and one spreading center. In contrast to cases like IST-4, the subduction here is slow enough to prevent a vanishing of the entire cold surface material. From $1.4 \cdot 10^{-3}$ until $1.8 \cdot 10^{-3}$ the downwelling proceeds uniformly with thin new lithosphere. The "a" frames in figure 7.32 show the convective pattern at the end of this subduction process. From $2.0 \cdot 10^{-3}$ until $2.9 \cdot 10^{-3}$ no significant subduction occurs, however, the spreading center remains clearly recognizable (figure $7.32 \mathrm{~b}$ ) and the surface velocity always jumps above the spreading center (see the surface velocities in figure 7.32). Around the time $2.9 \cdot 10^{-3}$ subduction starts again (figure $7.32 \mathrm{c}$ ) and a statistically stable convection pattern with homogeneous surface velocity in an Earth-like order of magnitude is finally developed (figure $7.32 \mathrm{~d}$ ).

\subsubsection{Summary and Discussion}

The following results are obtained in this section:

(1) It is demonstrated that the episodic subduction of the entire lithosphere, which is usually obtained for broken lid models with high values of damage source $a$ and sink $b$, can significantly be inhibited by the introduction of a depth-dependent viscosity. Appropriate values for the viscosity increase with depth are a factor of hundred for an exponential increase or a factor of ten for a jump at the $660 \mathrm{~km}$ discontinuity. For these values the permanent presence of strongly localized LVZs at subduction zones and passive spreading centers becomes possible. In consequence the surface velocities in between remain very uniform. Subduction shows some asymmetry, however, this asymmetry is not very developed since $a \approx a_{\text {break }}$ is used in these examples. Especially with a viscosity jump at the $660 \mathrm{~km}$ discontinuity large plates with an extension of about $8000 \mathrm{~km}$ are observed.

(2) Transfered to the "real" Earth these experiments clearly demonstrate the importance of the depth-dependence of viscosity onto the structure of plate tectonics. 


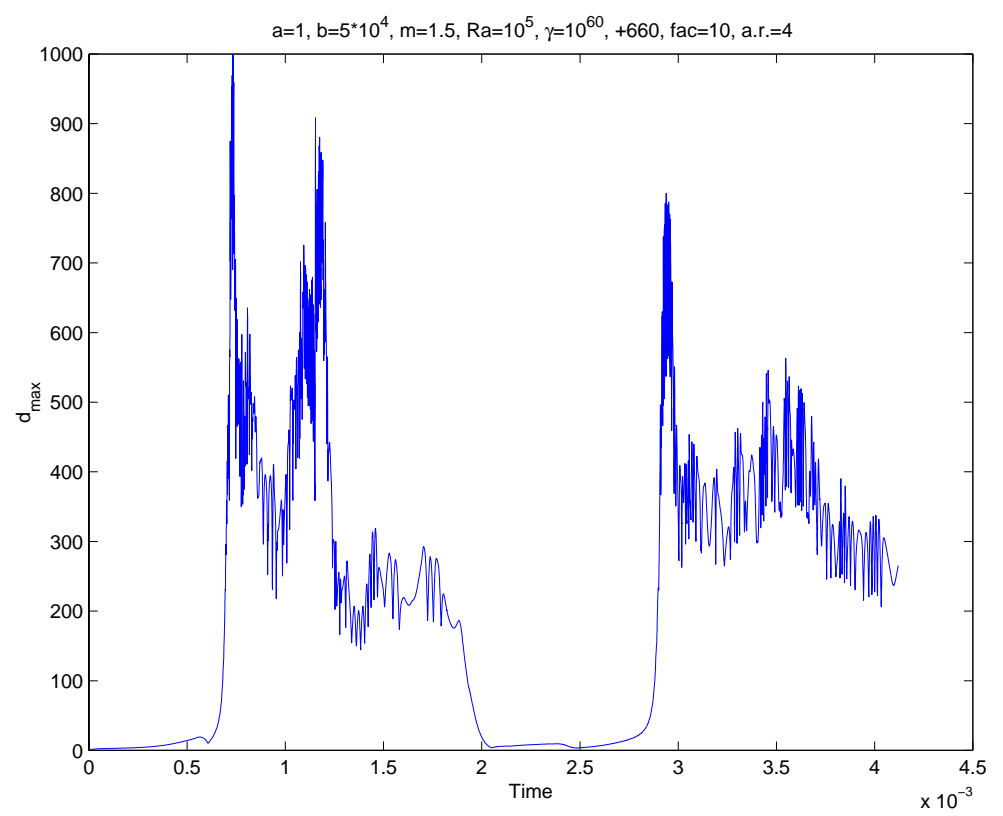

Figure 7.30: Maximum damage versus time for case DD-9

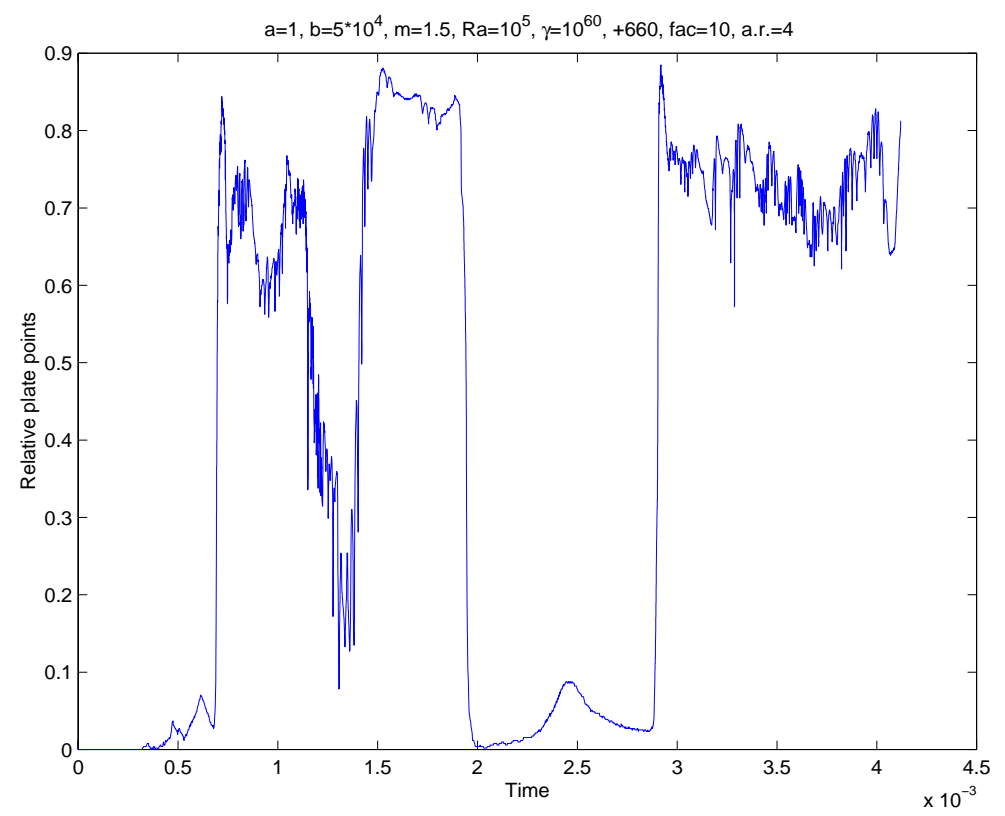

Figure 7.31: Relative plate points versus time for case $D D-9$ 

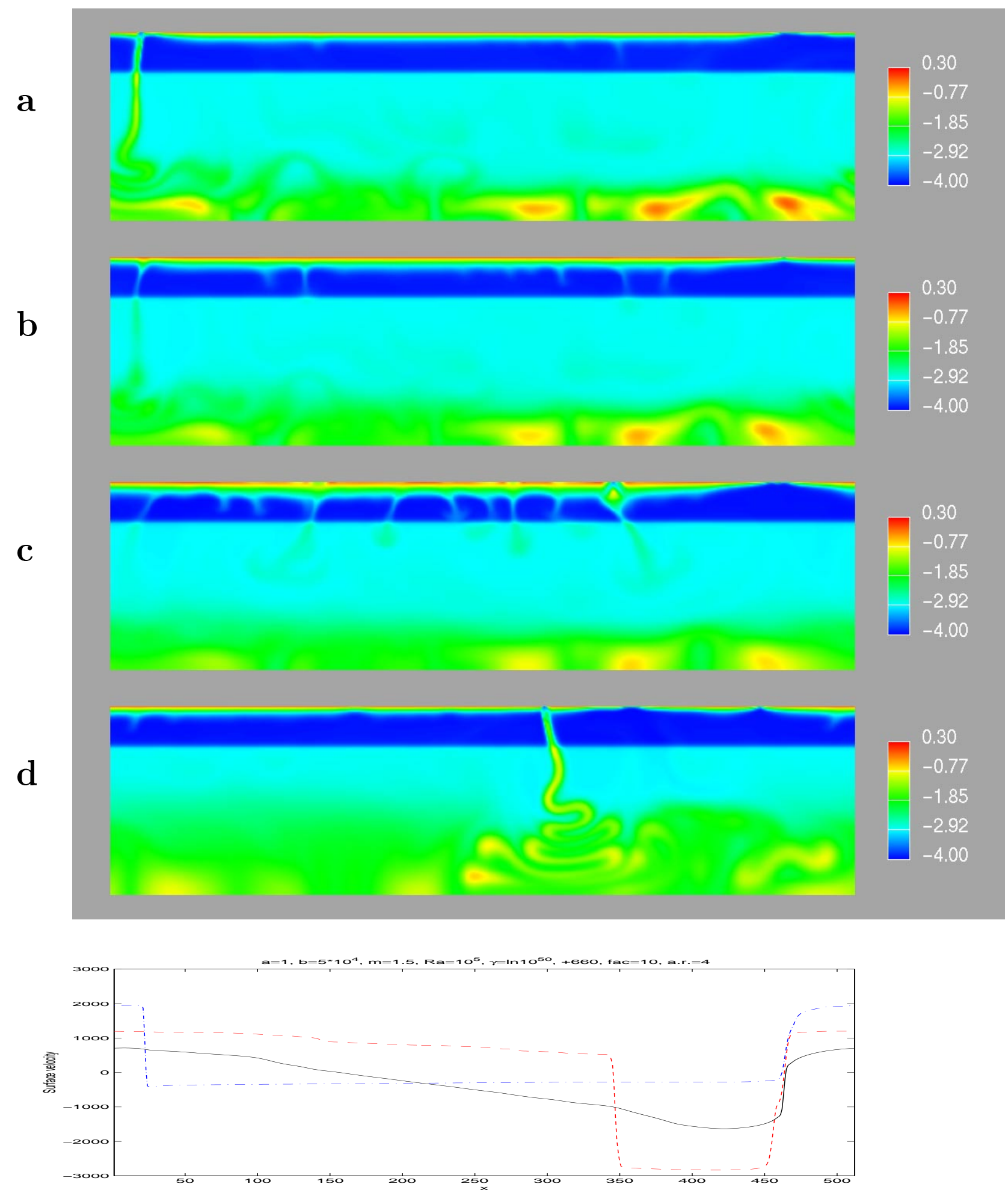

Figure 7.32: Viscosity and surface velocity snapshots for case DD-9 at times $1.79 \cdot 10^{-3}$ $\left(\right.$ dashed + dotted, a), $2.18 \cdot 10^{-3}$ (solid, surf. vel. ten times increased, b), $2.90 \cdot 10^{-3}$ (dashed, c) and $4.12 \cdot 10^{-3}(d)$. 
(3) My previous estimates about the breaking parameters $a_{\text {break,Earth }}$ and $b_{\text {break,Earth }}$ (see section 7.2) have to be modified. These estimates included a simultaneous increase of $a_{\text {break,Earth,1 }}$ and $b_{\text {break,Earth }, 1}$ to obtain LVZs of an Earth-like width. However, this is based on the measure of LVZs in simulations far away from their final statistical steady state (case BR4-2). If instead the steady state of case DD-4 for example is used to obtain a more realistic estimate a decrease of $a_{b r e a k, E a r t h, 3}$ and $b_{b r e a k, E a r t h, 3}$ by at least a factor of four is necessary. New estimates are therefore:

$$
a_{\text {break }, \text { Earth }, 4} \approx 50
$$

and

$$
b_{\text {break }, \text { Earth }, 4} \approx 5 \cdot 10^{4}
$$

for $m=2.0$. Transfered into physical quantities that means that 125 percent of the total power per volume in Earth are needed to reduce the grain size in the lithosphere (from $a$ ) and that the decay time for passive faults is about 6 Million years (from $b$ ). Of course, $a$ and $b$ are still too high, however, some of my assumptions are very crude (the size of the scaling damge $d_{0}$ in equation 7.14 or the $m$ dependence of $a_{b r e a k, E a r t h}$ for example) and $a$ and $b$ values reduced by another order of magnitude are probably perfectly reasonable.

(4) Regarding the result of case DD-8 the previous estimate for the upper boundary of $a$ for Earth can be increased to roughly

$$
a_{\text {upper,Earth }, 2}<12 \cdot a_{\text {break,Earth }}
$$




\section{Chapter 8}

\section{Summary and Discussion}

This chapter summarizes the results obtained in these thesis and discusses similarities and differences with previous publications.

\subsection{Influence of the damage input parameters}

Presents three convective regimes to classify my simulations and discusses the influence of $a ; a$ and $b ; m$ based on these regimes.

\subsection{Plate-like behavior in the simulations}

The criteria for plate-like behavior developed in subsection 2.2.1 are used to evaluate my results.

\subsection{Discussion and Conclusion}

Compares my results to the previous ones introduced in section 2.2 and gives a final statement to my rheological model. 


\subsection{Influence of the damage parameters}

The most important aim of these thesis was to systematically investigate the influence of the damage source parameter $a$, the sink parameter $b$ and the exponential dependence of viscosity on damage $m$ onto the convective structures in the computational domain.

For a better understanding the result of the simulations are classified into three different convective regimes. In this section the definitions of these regimes are briefly repeated and the influence of the parameters $a, a$ and $b$ and $m$ with respect to these regimes are presented.

\subsubsection{Convective regimes}

Three different convective regimes can be identified in the presented simulations:

Low Damage Regime:

The convective structure in the computational domain is not significantly influenced by the damage over at least two mantle overturn times.

VARiable Damage Regime:

This regime shows strong temporal variations of the damage which are usually connected with significant changes in the intensity of downwellings. Three types of this regime can be distinguished:

- Type II: Damage changes from very small to intermediate values. LVZs at different positions (above downwellings and at passive spreading center, for example) are not connected.

- Type IV: Damage changes from intermediate to high values. LVZs at different positions become temporarily connected.

- Type VI: This is a combination of type II and IV: Damage increases from low to very high values, LVZs develop at different positions and become connected to each other.

To distinguish this regime from transitions between the Low Damage Regime and the following Homogeneous Damage Regime I define that at least two cycles of episodic behavior have to be present for this Variable Damage Regime.

Homogeneous Damage Regime:

In this regime damage has intermediate values and influences the viscosity significantly for at least two mantle overturn times at the same position.

\subsubsection{Influence of the damage source parameter $a$}

In general, increasing $a$ increases the amount of damage especially within the lithosphere. This typically enlarges the asymmetry of subduction but also the time-dependence of 
convection.

However, depending on the heating mode some more specific results are obtained:

\section{Bottom heated models}

On increasing $a$ the following sequence of convective regimes was found in all simulations: Low Damage Regime, Variable Damage Regime type II, Homogeneous Damage regime, Variable Damage Regime type IV. The asymmetry of subduction is caused by strong differences in the strength of convection in the two convective cells. These convective differences alternate in time.

\section{Internally heated models}

An obvious correlation between an increase of $a$ and a sequence of convective regimes was not found for internally heated models. Nevertheless for larger $a$ convection in the Variable damage regime type IV is preferred over the Low Damage Regime, the Variable Damage Regime type II or even the Homogeneous Damage Regime.

The increase of asymmetry in a subduction zone is usually related to a growing difference in the sizes of the two related plates. For high $a$ values at least one of these two plates can become very small (i.e. a few hundred km long).

For models, which would show convection in the sluggish lid or in the low viscosity contrast regime for vanishing damage, the Homogeneous Damage Regime is only obtained when no passive spreading center is present.

Models, which would convect in the stagnant lid regime without damage influence, show a well defined transition between the Low Damage Regime and the other Regimes. This transition is marked by the value $a_{\text {break }}$ which has been determined for different values of $R a$ and $\gamma$ in section 7.2. The principal increase of $a_{b r e a k}$ with increasing viscosity contrast and decreasing Rayleigh number can be explained semi-heuristically with a dependence on the maximum difference in the surface velocity $\Delta u_{\text {surf }}$ and the thickness of the thermal boundary layer $D: a_{\text {break }} \sim\left(D^{3 / 2}\right) /\left(\Delta u_{\text {surf }}^{2}\right)$.

Simulations with $a \geq a_{\text {break }}$ show always both, passive spreading centers and subduction zones. Fast episodic subduction of the entire lithosphere into the mantle followed by its slow re-growth is the preferred convection pattern. The Homogeneous Damage Regime can only be established if either no significant self-focusing of LVZs is accepted or if an additional depth-dependence of viscosity is integrated into the model.

For $a \approx a_{\text {break }}$ subduction is usually quite symmetric, but $a=4 a_{b r e a k}$ already shows significant asymmetry. However, for $a=4 a_{\text {break }}$ the subduction angle is typically around 120 degrees instead of 60 and the plate size is reduced compared to $a \approx a_{\text {break }}$.

The introduction of an additional depth-dependence of viscosity into the model allows the development of a stable Homogeneous Damage Regime convection with localized LVZs above the downwelling and localized passive spreading centers. An exponential increase 
of viscosity with depth by a factor of around hundred or a viscosity jump at the 660 $\mathrm{km}$ discontinuity by a factor of ten are appropriate choices. With the latter possibility a plate-length of at least $8000 \mathrm{~km}$ can be reached.

The following breaking parameters can be estimated for Earth's stagnant lid: $a_{\text {break,Earth }} \approx 50$ and $b_{\text {break,Earth }} \approx 5 \cdot 10^{4}$ assuming $m=2.0$ and a viscosity jump by a factor of ten at the $660 \mathrm{~km}$ discontinuity. The corresponding physical interpretation for $a_{b r e a k, E a r t h}$ is that 125 percents of the total power per volume in Earth are needed to reduce the grain size in the lithosphere. The decay time of passive faults deduced from $b_{\text {break,Earth }}$ is about 6 Million years. Both estimations $a_{b r e a k, E a r t h}$ and bbreak, Earth therefore appear to be at least an order of magnitude too high. Taking the quality of these estimations into account this is, however, not necessarily an argument against my model.

From calculations with $a>a_{\text {break }}$ I also extrapolated $a_{\text {Earth }}<12 \cdot a_{\text {break,Earth }}$ as a crude approximation for an upper boundary of $a$ on Earth.

\subsubsection{Influence of advection and $m$}

The influence of damage advection in the box is determined by the absolute sizes of $a$ and $b$ ( $a / b$ constant $)$ and also by $m$.

The local influence of damage advection mostly effects the LVZs: For low values of $a$ and $b$ the viscosity in LVZs is comparatively high, the LVZs are quite extended and have a linear structure. Increasing $a$ and $b$ decreases the viscosity inside the LVZs and also their extension. Their structures become "v"-like. When $a$ and $b$ exceed some critical values (which also depend on $a / b$ and $m$ ) self-focusing behavior of the LVZs is obtained. An appropriate measure to empirically determine these critical values is the maximum value of the second strain invariant, which significantly increases when self-focusing starts.

The local changes in the structures of LVZs are also able to influence the convection pattern in the entire box. An increase in $a$ and $b$ basically promotes a stronger time-dependence of convection. For bottom heated systems a transition from the Homogeneous Damage Regime into the Variable Damage Regime type IV is possible, for internally heated systems the Variable Damage Regime type IV is observed instead of type II.

The parameter $m$ acts in a similar way. In my simulations it is basically used to ensure self-focusing behavior in the box. An appropriate value is $m=1.5$.

\subsection{Plate-like behavior in the simulations}

Appropriate plate-like behavior including most of the features observed on Earth is only obtained for internally heated models with a broken stagnant lid and a depth-dependent viscosity.

With respect to the criteria for plate-like behavior developed in the introduction (see section 2.1) the simulations produce the following more detailed results: 


\subsubsection{High viscosity of the surface material}

Because of the temperature-dependence of the viscosity a high-viscous surface is present in nearly all calculations. Exceptions are the steady states for the sluggish lid models where the temperature inside the box significantly decreases during the calculation (chapter 6) and the highly episodic broken lid simulations after all surface material has suddenly subducted into the mantle (see section 7.3).

\subsubsection{Localized mid ocean ridges and subduction zones}

In all simulations with significant damage influence LVZs are obtained above the downwellings. A decrease of the damage advection by a simultaneous increase of $a$ and $b$ or of $m$ always allows to focus these LVZs as much as needed.

In contrast, the development of (focused) spreading centers is not self-evident in those models. In fact, four different situations are observed:

1. Although focused LVZs above the downwelling are present, no passive spreading is developed. Examples are models which would be in the sluggish lid regime without damage influence ("ISL" series, chapter 6).

2. A spreading center is developed, but it does not remain focused. Examples are bottom heated cases in the variable damage regime type IV (see case A2-6) or internally heated cases with a broken stagnant lid but no depth-dependent viscosity (case IST4).

3. A permanent spreading center is developed, but is not focused, although the LVZ above the downwelling is comparatively narrow. Examples are case A2-3 for bottom heated cases or IST-1 for internal heating.

4. A localized spreading center is developed and remains focused for a long time. Examples are some cases in the homogeneous damage regime, like case M0-4 for bottom heated models or case DD-4 with depth-dependent viscosity for internally heated models.

The fourth situation is, of course, the most Earth-like. For internally heated models it is only obtained with depth-dependent viscosity.

\subsubsection{Surface velocity}

A plate-like surface velocity, which is uniform everywhere except for jumps at mid ocean ridges and subduction zones, depends on well developed LVZs above downwellings and at mid ocean ridges. The lower the viscosity inside the LVZs and the smaller these zones the better is the plate-like behavior of the surface velocity. The results discussed in the previous section are therefore also relevant for the surface velocity.

In my models a non-dimensional velocity of 1000 corresponds to a real velocity of about one centimeter per year. Except for some episodic peaks, the surface velocity in most of my models is too low (around some mm/year). However, these values seem acceptable, since I used Rayleigh numbers and temperature-dependences of the viscosity which are below the values estimated for Earth. 
A surface velocity constant over long periods of time can only be attained in the Homogeneous Damage Regime. The most Earth-like models are therefore again the internally heated broken lid models with a depth-dependent viscosity, where this regime prevails.

\subsubsection{Size of the plates}

The size of developing plates in (non-highly-episodic) bottom heated models is basically determined by the size of the underlying mantle convection cells. In the simulation presented here the plate-size is therefore around $3000 \mathrm{~km}$.

For internally heated models an increase in the damage source parameter $a$ increases the differences in the length of different plates. In a simulation with a broken lid and $a \approx a_{\text {break }}$, for example, the two plates forming a subduction zone have usually similar extensions. In contrast, simulations at higher $a$ show one small fast-moving plate and one large slowmoving plate at a subduction zone.

The introduction of a depth-dependent viscosity in an internally heated broken lid model allows the development of stable plates with a size of at least $8000 \mathrm{~km}$ for $a \approx a_{\text {break }}$.

\subsubsection{Asymmetry of subduction}

For both heating modes an increase of $a$ also increases the asymmetry of subduction.

However, for bottom heated cases the asymmetry of the subduction velocity of both plates and the subduction angle are strictly related. Both are caused by differences in the intensities of convection in the two cells, and since these differences oscillate the asymmetry is highly variable in time.

For internally heated models this strong correlation between the asymmetry of the surface velocities and the subduction angle is not obtained. Cases ISL-1 or ISL-4, for example, show strong temporary differences in the surface velocities of two subducting plates, but the subduction occurs vertically.

The quantity which basically influences the asymmetry in the plate velocities at a subduction zone is the length of the plates when the subduction develops. The smaller the plate the faster its velocity. A larger $a$ promotes self-focusing behavior and therefore increases small initial differences in the plate velocities. Once a significant asymmetry is established it is maintained by the positive feedback between the plate and the underlying mantle. However, this mechanism also prevents the subduction of a passive spreading center.

Asymmetry in terms of the non-vertical penetration of lithospheric material into the mantle (i.e. a subduction angle different from 90 degrees) is obtained for three different reasons in the simulations:

(1) Differential movement between mantle and lithosphere (cases IST-2 or ISL-3),

(2) Mantle inhomogeneities from previous subduction events (no example shown here)

(3) Sucking of the already subducted material beneath the fast moving plate (case IST-3)

Mechanism (1) is probably relevant for Earth and, moreover, supported by a depth- 
dependent viscosity. Mechanism (2) is mainly obtained in simulations with significant timedependence. However, it may also be relevant for Earth. The third mechanism (3) naturally produces the wrong subduction angle and is therefore highly non-Earth-like.

\subsection{Discussion and Conclusion}

\subsubsection{Similarities with previous models}

Many of the features that other authors describe for yield stress rheologies or damage dependent rheologies were also observed in my experiments.

Moresi's and Solomatov's (1998) results for a bottom heated model with yield stress rheology are qualitatively comparable to the first three regimes obtained for bottom heated cases: Their stagnant lid regime is equivalent to my Low Damage Regime, the episodic overturn regime is analogous to my Variable Damage Regime type II and the mobile lid regime is comparable to the Homogeneous Damage Regime without self-focusing of the LVZs.

In a similar way Trompert's and Hansen's (1998) results can be classified into the Variable Damage Regime type II.

Stein (2000) showed that the introduction of an exponential depth-dependence of the viscosity reduces the episodic behavior for Trompert's and Hansen's models and produces permanent subduction of the lithosphere. She found an increase of viscosity by a factor of 40, to give the best results for her models. This is consistent with the results obtained here. However, my calculations also show that a much lower viscosity jump at the $660 \mathrm{~km}$ transition produces probably better results.

For a decreasing yield stress Tackley (2000b, 2000c) found the following order of convective regimes for internally heated models: stagnant lid regime, episodic regime and mobile lid regime. This is consistent with my regime order for increasing $a$, i.e. the Low Damage Regime, the Variable Damage Regime type II and the Homogeneous Damage Regime, all without self-focusing. This correlation makes sense since Tackley's yield stress corresponds to my possible maximum stress in the material, which decreases with increasing $a$ (see section 3.3). Moreover, I obtain a new time-dependence for further increased $a$ which has no counterpart in Tackley's simulations. However, since the self-focusing behavior becomes

relevant at these large $a$ values a similar behavior may not be possible for yield stress rheologies.

An exponential viscosity increase with depth by a factor of ten with depth was found to have minor influence on Tackley's convective system. This is confirmed here. Significant improvements are only obtained with a stronger depth-dependence.

Tackley also reported that his results become more Earth-like, when an asthenosphere it included into his model, even when it is restricted to regions around the spreading center. Although this appears comparable to my viscosity-jump-results, the reasons for the introduction of these two features into the models are very different: Tackley needs the 
asthenosphere to obtain focused spreading centers, which is not necessary for my rheology. Similar to Stein I need the depth-dependent viscosity to inhibit the episodic behavior and to increase the asymmetry in the propagation of subducted material into the mantle.

The fragmentation of plates as observed by Tackley for damage-dependent rheologies can not be confirmed in general. Although very small plates are obtained for $a>4 \cdot a_{\text {break }}$ or higher, plates as large as $8000 \mathrm{~km}$ are observed for $a \approx a_{\text {break }}$ and if the viscosity is depth-dependent.

The existence of "v" shaped LVZs was also observed in numerical experiments from Montesi and Zuber (2001) who investigated power law rheologies with negative exponents. It is also consistent with the existence of conjugated shear zones on Earth.

\subsubsection{Conclusion}

In these thesis a rheology was investigated, where the viscosity not only depends on temperature and depth but also on a damage parameter. Temporal evolution of the damage was described by an additional equation featuring source, sink and advection of damage.

The aim of the new rheology was to improve the plate-like behavior of the lithospheric material in numerical simulations. In particular it was expected to (1) produce narrow spreading centers and low viscosity zones (LVZs) above downwellings and therefore piecewise uniform surface velocities, (2) increase the asymmetry of subduction zones and (3) create long living passive fault structures.

Systematical parameter studies for different convective regimes demonstrated that some of these expectations were indeed fulfilled.

- This rheology is able to develop sharp and self-focused passive spreading centers and LVZs at downwellings. In consequence highly uniform surface velocities are obtained.

- It can produce significant asymmetry in both, the velocity of the two plates at a subduction zone and the penetration of the subducted material in the mantle.

- In addition, the simulations demonstrate the importance of a depth-dependent viscosity.

Although these features show that the rheology employed here is significantly more successful in reproducing plate-like behavior than previously published models, some important features of Earth's plate tectonics are still not explained appropriately:

- A complete one-side subduction observed on Earth is not found here.

- If significant asymmetry is obtained in simulations the length of the corresponding plates is often too small and the subduction angle is sometimes wrong. 
- The subduction of spreading centers is not possible.

- Estimations for the damage source $a_{\text {Earth }}$ and sink $b_{\text {Earth }}$ needed to break a stagnant lid on Earth are significantly too high.

- Long living passive faults are not obtained.

Nevertheless, in my opinion this kind of damage-, temperature- and depth-dependent rheology has a good potential for further explaining plate tectonics on Earth. The results from Bercovici $(1996,1998)$ about the evolution of transform faults and Tackley (2000c) support these expectations. However, the introduction of further geophysical aspects like the elasticity of plates or the brittle breaking process for the upper 10 or $20 \mathrm{~km}$ of the lithosphere might be advisable. 


\section{Appendix A}

\section{A.1 Non dimensional quantities in the Hydrodynamic equations}

The following section describes how the dimensional quantities in the hydrodynamic equations are non-dimensionalized. The dimensional quantities are marked with the ${ }^{\sim}$ sign. For the bottom heated system we use:

$\tilde{x}=\frac{x}{h}$

$\tilde{z}=\frac{z}{h}$

$\tilde{t}=\frac{t \kappa_{0}}{h^{2}}$

$\tilde{T}=\frac{T-T_{0}}{T_{1}-T_{0}}$

$\tilde{d}=\frac{d}{d_{0}}$

$\tilde{p}=\frac{\left(p-g \rho_{0} z\right) h^{2}}{\kappa_{0} \eta_{0}}$

$\tilde{\eta}=\frac{\eta}{\eta_{0}}$

$\tilde{\kappa}=\frac{\kappa}{\kappa_{0}}$

$\tilde{\rho}=\frac{\rho}{\rho_{0}}$

$x$ and $z$ are the horizontal and vertical spatial coordinates, $t$ is the time, $T$ the temperature, $d$ the inverse grain size, $p$ the pressure, $\eta$ the viscosity $\kappa$ the thermal diffusivity $\tilde{\rho}$ the density.

$g$ is the gravitational acceleration, $\alpha$ the thermal expansivity, $\rho_{0}$ the density at the surface $h$ the height of the Earth mantle, $\kappa_{0}$ the constant thermal diffusivity in the box 
$\eta_{0}$ the viscosity at the surface and for a non-dimensional damage of one.

$T_{0}$ is the surface temperature

$T_{1}$ the bottom temperature and

$d_{0}$ a typical grain size at the surface.

Typical values for scaling parameters in the Earth's mantle are:

\begin{tabular}{l|l|r|l} 
Quantity & Meaning & $\begin{array}{r}\text { Value for the } \\
\text { Earth's mantle }\end{array}$ \\
\hline$g$ & gravity acceleration & 10 & $\mathrm{~m} \cdot \mathrm{s}^{-2}$ \\
$\alpha$ & thermal expansivity & $2 \cdot 10^{5}$ & $\mathrm{~K}-1$ \\
$\rho_{0}$ & Density & $4 \cdot 10^{3}$ & $\mathrm{~kg} \cdot \mathrm{m}^{-1}$ \\
$h$ & Thickness of the mantle & $2.9 \cdot 10^{6}$ & $\mathrm{~m}$ \\
$\kappa_{0}$ & thermal diffusivity & $1 \cdot 10^{-6}$ & $\mathrm{~m}^{2} \cdot \mathrm{s}-1$ \\
$\eta_{0}$ & Viscosity on the & $1 \cdot 10^{24}$ & $\mathrm{~Pa} \cdot \mathrm{s}$ \\
$T_{1}-T_{0}$ & top of the box & & \\
& temperature difference & & \\
& between top and & & $\mathrm{K}$ \\
$d_{0}$ & bottom of the box & $10^{3}$ & $\mathrm{~m}$ \\
& Typical inverse grain & & \\
$q$ & size at the surface & $5 \cdot 10^{-9}$ & $\mathrm{~W} \cdot \mathrm{m}^{-3} \cdot \mathrm{s}^{-1}$ \\
$c_{p}$ & rate of internal heat generation & $1.25 \cdot 10^{3}$ & $\mathrm{~W} \cdot \mathrm{kg}^{-1} \cdot \mathrm{K}^{-1}$
\end{tabular}

Table A.1: Values for the scaling parameters in the Earth's mantle

The Rayleigh number obtained from the non-dimensionalization of the hydrodynamic equations for a bottom heated model is:

$$
R a_{b h}=\frac{g \alpha \rho_{0}\left(T_{1}-T_{0}\right) h^{3}}{\kappa_{0} \eta_{0}}
$$

For an internally heated system I use

$\tilde{q}=q / q_{0}$ to define the non-dimensional temperature as

$\tilde{T}=\frac{\rho c_{p} \kappa_{0}\left(T-T_{0}\right)}{h^{2} q_{0}}$, where

$q$ is the rate of internal heating per unit volume and unit time and $c_{p}$ the specific heat.

The Rayleigh number for an internally heated system is therefore:

$$
R a_{i h}=\frac{\alpha g q_{0} h^{5}}{\kappa_{0}^{2} \eta_{0} c_{p}}
$$




\section{Acknowledgments}

First at all, I wish to thank Prof. Dr. Ulrich Christensen for supervising these thesis.

Prof. Dr. David Bercovici made one fantastic year at the University of Hawaii possible. A special thank to him for many scientific suggestions as well as for teaching me how to become really cynical.

The professors Tilgner, Lube, Werner, Glatzel and Kneer most kindly accepted the great honor to listen to the defense of these thesis.

Johannes Wicht, Georg Kaufmann, Ursula Wuehlner and Ajay Manglik significantly improved the structure of this work.

Another "thank you" to all the kind people in the Institute of Geophysics who always believed (and hoped) that I will finish my $\mathrm{PhD}$ some day.

Clearly, I would not have survived especially the last few weeks without the help of Janio and my family. Thanks for the great support.

My $\mathrm{PhD}$ was also supported by scholarships from the University of Goettingen, the German federal state Niedersachsen, the DAAD, the University of Hawaii and the Graduiertenkolleg "Stroemungsinstabilitaeten". Tanks a lot to all of them. 


\section{Bibliography}

[1] Auth, C., Harder, H. Multigrid solution of convection problems with strongly variable vsicosity, Geophys. J. Int. 1999, 137, 793-804

[2] Batchelor,G.K.: An introduction to fluid dynamics, Cambridge University Press, 1994

[3] Bercovici, D., A simple model of plate generation from mantle flow, Geophys. J. Int., 114, 635-650, 1993

[4] Bercovici, D., A source-sink model of the generation of plate tectonics from nonNewtonian mantle flow, J. Geophys. Res., 100, 2013-2030, 1995

[5] Bercovici, D. Plate generation in a simple model of litho-sphere-mantle flow with dynamic self-lubrication, Earth Planet. Sci. Lett., 144, 41-51, 1996

[6] Bercovici, D., Generation of plate tectonics from lithosphere-mantle flow and voidvolatile self-lubrication, Earth Planet. Sci. Lett., 154, 139-151, 1998

[7] Bercovici, D., Ricard, Y., Richards, M.A., The relation between Mantle Dynamics and plate tectonics: A primer, AGU Monograph on the History and Dynamics of Global Plate Motions, M. Richards, R. Gordon and R. van der Hilst (editors), American Geophysical Union, 2000

[8] Blankenbach,B. et AL.: A benchmark comparison for mantle convection codes, Geophys. J. Int. 98, 23-38, 1989

[9] Braun, J., Chery, J., Poliakov, A., Mainprice, D., Vauchez, A., Tomassi, A., Daignieres, M., A simple parametrization of strain localization in the ductile regime due to grain size reduction: A case study for olivine, JGR 104, B11, 2516725181, 1999

[10] Busse, F.H., Frick, H. Square-pattern convection in fluids with strongly temperature-dependent viscosity, J. Fluid Mech. 76, 741-754

[11] Christensen,U.R.: Convection with pressure- and temperatur-dependent nonNewtonian rheology, Geophys.J.R.astr.Soc.,77,343-384,1984 
[12] Christensen,U.R.: Time-dependent convection in elongated Rayleigh-Benard cells, Geophys. Research Letters, Vol. 14, No. 3, pp. 220-223, march 1987

[13] Christensen, U.R., Harder, H., Three-dimensional convection with variable viscosity, Geophys. J. Int., 104, 213-226, 1991

[14] Christensen,U.R.: Fixed hotspots gone with the wind, Nature 391, 739-740, 1998

[15] Evans, B., Kohlstedt,D. L., Rheology of rocks in Rock Physics and Phase Relations: A Handbook of Physical Constants, AGU Reference Shelf 3, American Geophysical Union, pp. 148-165, 1995

[16] Fowlwer, C.M.R. The solid Earth, Cambridge University Press, 1990

[17] Gurnis, M., Zhong, S., And Toth, J.: On the competing roles of fault reactivation and brittle failure in generating plate tectonics from mantle convection, in Richards, $\mathrm{M}$. A., Gordon, R., and van der Hilst, R., (editors), The History and Dynamics of Global Plate Motions,American Geophysical Union, Washington D.C., 73-94, 2000.

[18] Karato, S. Grain-size distribution and rheology of the upper mantle, Tectonophysics, $104,155-176,1984$

[19] Karato, S., Wu, P. Rheology of the upper mantle: A synthesis, Science, 260, 7 May, $771-778,1993$

[20] Kohlstedt, D.L., Evans, B., Mackwell, S.J. Strength of the lithosphere - Contraints imposed by laboratory experiments, J. Geophys. Res., 100, B9, 17587-17602, 1995

[21] Koren, B. Numerical methods for advection-diffusion problems, Vreugdenhil, C.B. (editor), Notes on numerical fluid mechanics 45, Vieweg, 1993

[22] Jin, D.H., Karato, S., Obata, M. Mechanisms of shear localization in the continental lithosphere - Inference from the deformation microstructures of peridotites from the Ivrea Zone, northwestern Italy, J. Struct. Geol, 20(2-3), 195-209, 1998

[23] Montesi, L.G.J., Zuber, M.T., Macroscale localisation instability: A semianalytical investigation into the generation of regularly spaced faults, submitted to J. Fluid Mech., 2001

[24] Molnar, P., Stock, J., Nature 327, 587-591, 1987

[25] Monnereau, M., Quere, S., Spherical shell models of mantle convection with tectonic plates, EPSL, 184, 575-587, 2001 
[26] Moresi,L., Solomatov, V., Mantle convection with a brittle lithosphere: Thoughts on the global tectonic style of the Earth and Venus, Geophys. J. Int., 133, 669-682, 1998

[27] Norton, I.O., Tectonics 14, 1080-1094 (1995)

[28] Pili, E., Ricard, Y., Lardeaux, J.M., Sheppard, S.M.F. Lithospheric shear zones and mantle-crust connections, Tectonophysics, 280(1-2), 15-29, 1997

[29] Ranalli, G., Rheology of the Earth,Chapman and Hall Publishers, London, 1995

[30] Ratcliff, J. T., Tackley, P., Schubert, G., Zebib, A. Transitions in thermal convection with strongly variable viscosity

[31] Richards, M.A., Lithgow-Bertelloni, C. ??? EPSL 137, 19-27, 1996

[32] Solomatov, V.S., Scaling of temperature dependent and stress dependent viscosity convection, Phys. Fluids, 7, 266-274, 1995

[33] Stein, C., Selbstkonsistente Ausbildung plattentektonischer Vorgaenge in einem Konvektionsmodell mit variabler Viskositaet, Diplomarbeit im Fach Physik, Westfaelische Wilhelms Universitaet Muenster, 2000

[34] TACKLEy, P.: Self-consistent generation of tectonic plates in three-dimensional mantle convection, Earth and Planetary Science Letters, 157, 9-22, 1998

[35] Tackley, P., The quest for self-consistent generation of plate tectonics in mantle convection models, AGU Monograph on the History and Dynamics of Global Plate Motions, M. Richards, R. Gordon and R. van der Hilst (editors), American Geophysical Union, 2000a

[36] Tackley, P.: Self consistent generation of tectonic plates in time-dependent, threedimensional mantle convection simulations 1. Pseudoplastic yielding, Geochem. Geophys. Geosyst., v 1, Aug. 232000 b

[37] TACKLEy, P.: Self consistent generation of tectonic plates in time-dependent, threedimensional mantle convection simulations 2. Strain weakening and asthenosphere, Geochem. Geophys. Geosyst., v 1, Aug. 23 2000c

[38] Tritton,D.C.: Physical fluid dynamics, Oxford science publications, 1995

[39] Trompert,R.A., Hansen,U.: The application of a Finite Volume Method to threedimensional flow problems in a highly viscous fluid with a variable viscosity, Geophys. Astrophys. Fluid Dynamics, Vol 83, pp. 261-291

[40] Trompert,R.A., Hansen,U., Mantle convection simulations with rheologies that generate plate-like behavior, Nature, 395, 686-689, 1998 
[41] VAN ANDEL, T.H. Seafloor spreading and platetectonics, in Understanding the Earth, Brown, G.C, Hawkesworth, C.J., Wilson, R.C.L. (editors), Cambridge University Press, 1992

[42] Vermeer, P.A. The orientation of shear bands in biaxial tests, Geotechnique, 40, 223-236, 1990

[43] Weertman, J., Weertman, J.R., High temperature creep of rock and mantle viscosity, Ann. Rev. Earth. Planet. Sci., 3 293-315, 1975

[44] Vissers, R.L.M., Drury, M.R., Strating, E.H.H., Spiers, C.J., VanderWAL, D. Mantle shear zones and their effect on lithosphere strength during continental breakup, Tectonophysics, 249(3-4), 551-572, 1996

[45] Weinstein, S.A., Olson, P.,Thermal convection with non-Newtonian plates, Geophys. J. Int., 111, 515-530, 1992

[46] Zhong,S., Gurnis,M., Mantle convection with plates and mobile, faulted plate margins, Science, 267, 838-843, 1995 


\section{$\underline{\text { Lebenslauf }}$}

28.06.1971 geboren in Essen, deutscher Staatsangehoeriger

Schule:

1977-1981 Besuch der Grundschule Essen-Burgaltendorf

1981-1990 Schueler des humanistischen Burggymnasiums Essen

31.05.1990 Abitur

Zivildienst:

01.07.1990 Beginn des Zivildienstes auf der Pflegestation des antroposophischen Bettina-von-Arnim-Altenheimes in Essen-Stadtwald

Studium:

01.10.1991 Aufnahme des Studiums der Physik und der Mathematik an der Georg-August-Universitaet Goettingen

18.10.1993 Vordiplom Physik

22.04.1994 Vordiplom Mathematik

01.10.1995 Beginn der Diplomarbeit bei Prof. Dr. U. Christensen am Institut fuer Geophysik der Universitaet Goettingen Thema: "Ein Mehrgitterverfahren zur Loesung zweidimensionaler Konvektionsprobleme mit variabler Viskositaet"

27.06.1997 Diplom Physik

Job:

01.09.1997 Mitarbeit im magnetotellurischen und seismologischen Eifel-Plume-Projekt der Universitaet Goettingen

Promotion:

01.04.1998 Beginn der Promotion bei Prof. Dr. U. Christensen

Thema: "Plate tectonics in computational simulations of terrestrial mantle convection with grain-size-dependent rheology"

09.01.2000 Beginn eines einjaehrigen Aufenthaltes an der University of Hawaii in Honolulu im Rahmen der Promotion

10.07.2000 Teilnahme an einer Summerschool ueber Parallelcomputing im NASA Goddard Space Flight Center

31.12.2001 Voraussichtliches Ende der Promotion 